Judith Safford

\title{
Staatsverschuldung im Vereinigten Königreich
}




\section{Judith Safford}

\section{Staatsverschuldung im Vereinigten Königreich}

Der keynesianische Ansatz, Budgetdefizite als Instrument einer aktiven Stabilisierungspolitik einzusetzen, wurde in den 90er Jahren abgelöst durch monetaristisch geprägte Strategien zur Konsolidierung der Staatsfinanzen. Die Konservative Regierung unter Margaret Thatcher nahm im Vereinigten Königreich bereits Anfang der 80er Jahre eine Vorreiterrolle bei diesem Politikwechsel ein. Die Arbeit analysiert die damit verfolgte Strategie zur Gesundung der Staatsfinanzen über den Zeitraum von 1979 bis 1994. Es zeigt sich, daß der damalige Abbau der öffentlichen Defizite und somit der Schuldenquote größtenteils auf einmaligen Einnahmen aus der Privatisierungspolitik, den Steuererlösen aus dem Nordseeöl und dem Verteilungsmechanismus der Inflation beruhten. Nach dem weitgehenden Wegfall dieser Finanzierungsquellen wurde Anfang der 90er Jahre erneut auf die Kreditfinanzierung zurückgegriffen. Die für eine dauerhafte Konsolidierung der Staatsfinanzen erforderliche Senkung der Ausgabenlast konnte trotz einiger Reformen gegen die Wählermehrheit nicht durchgesetzt werden. Ein wesentliches Hindernis dabei scheint eine im Zuge der Politikreformen zunehmende Ungleichheit der Einkommensverteilung zu sein. Dies erhöht die Nachfrage nach Ausgaben für Verteilungszwecke und konterkariert somit die Konsolidierungsbemühungen.

Judith Safford wurde 1960 in London geboren und wuchs dort auf. Nach einigen Jahren kaufmännischer Berufstätigkeit nahm sie 1985 das Studium der Volkswirtschaftslehre an der Universität in Freiburg im Breisgau auf. Nach dem Diplomstudium arbeitete sie als wissenschaftliche Mitarbeiterin am Institut für Finanzwissenschaft der Universität Freiburg und promovierte 1997 bei Professor Dr. Hans-Hermann Francke. 
Staatsverschuldung im Vereinigten Königreich 


\section{FINANZWISSENSCHAFTLICHE SCHRIFTEN}

Herausgegeben von den Professoren

Albers, Krause-Junk, Littmann, Oberhauser, Pohmer, Schmidt

Band 86

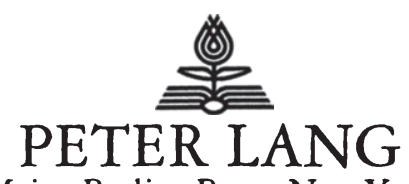

Frankfurt am Main · Berlin · Bern · New York · Paris · Wien 


\section{Judith Safford}

\section{Staatsverschuldung im Vereinigten Königreich}

Die öffentliche Verschuldung unter der Konservativen Regierung von 1979-1994

Ursachen und Auswirkungen

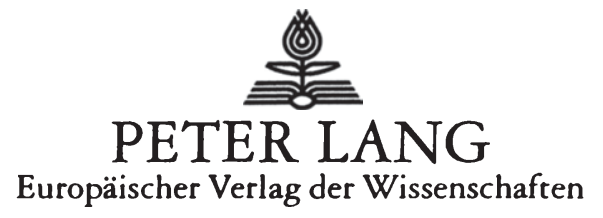


Die Deutsche Bibliothek - CIP-Einheitsaufnahme

Safford, Judith:

Staatsverschuldung im Vereinigten Königreich : die öffentliche Verschuldung unter der Konservativen Regierung von 19791994. Ursachen und Auswirkungen / Judith Safford. - Frankfurt am Main ; Berlin ; Bern ; New York ; Paris ; Wien : Lang, 1998 (Finanzwissenschaftliche Schriften ; Bd. 86) Zugl.: Freiburg (Breisgau), Univ., Diss., 1997 ISBN 3-631-33401-X

Open Access: The online version of this publication is published on www.peterlang.com and www.econstor.eu under the international Creative Commons License CC-BY 4.0. Learn more on how you can use and share this work: http://creativecommons. org/licenses/by/4.0.

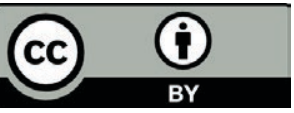

This book is available Open Access thanks to the kind support of ZBW - Leibniz-Informationszentrum Wirtschaft.

\author{
D 25 \\ ISSN 0170-8252 \\ ISBN 3-631-33401-X \\ ISBN 978-3-631-75199-2 (eBook) \\ (C) Peter Lang $\mathrm{GmbH}$ \\ Europäischer Verlag der Wissenschaften \\ Frankfurt am Main 1998 \\ Alle Rechte vorbehalten.
}

Das Werk einschließlich aller seiner Teile ist urheberrechtlich geschützt. Jede Verwertung außerhalb der engen Grenzen des

Urheberrechtsgesetzes ist ohne Zustimmung des Verlages

unzulässig und strafbar. Das gilt insbesondere für

Vervielfältigungen, Übersetzungen, Mikroverfilmungen und die Einspeicherung und Verarbeitung in elektronischen Systemen.

Printed in Germany 124567 


\section{Verdankungen}

Die vorliegende Dissertation entstand während meiner Tätigkeit als wissenschaftliche Assistentin am Institut für Finanzwissenschaft der Albert-LudwigsUniversität, Freiburg im Breisgau. Die kreative Atmosphäre und die Diskussionsbereitschaft meiner dortigen Kollegen haben ganz entscheidend zu dieser Arbeit beigetragen. Insbesondere habe ich viel von den kritischen Anregungen und der unermüdlichen Unterstützung von PD Dr. Jochen Michaelis, aber auch von Dr. Knut Blind und Dr. Dierk Brandenburg profitieren können. Ihnen möchte ich an dieser Stelle von ganzem Herzen meinen Dank aussprechen.

Eben so wichtig für die Entstehung der Arbeit war meine Familie. Sei es bei der Korrektur von sprachlichen Ungenauigkeiten, der Betreuung meines Sohnes oder durch Unterstützung im Familienhaushalt haben mich mein Mann Jürg Meyer, Sonja Meyer und Nancy Safford vor allem in der Endphase der Arbeit entscheidend entlastet. An sie geht mein besonderer Dank.

Schließlich möchte ich mich beim Erstgutachter der Arbeit und meinem Doktorvater Herrn Professor Dr. Hans-Hermann Francke bedanken. Sein persönliches Engagement für das Gelingen der Arbeit hat mich stets motiviert. Ferner möchte ich Herrn Professor Dr. Bernd Raffelhüschen, dem Zweitgutachter, für seine Diskussionsbereitschaft und seine Anregungen herzlich danken. Für die Aufnahme meiner Arbeit in die Reihe "Finanzwissenschaftliche Schriften" ist Herr Professor Dr. Alois Oberhauser verantwortlich, dem ich dafür zu guter letzt meinen Dank aussprechen möchte.

Mittelhäusern bei Bern im Januar 1998 
Judith Safford - 978-3-631-75199-2

Downloaded from PubFactory at 01/11/2019 07:12:45AM

via free access 


\section{Vorwort}

Die Wirtschaftspolitik und die Reformen der Regierung Thatcher während der 80er Jahre gelten für Ökonomen und Politiker vieler Länder als Vorbild. Nicht zuletzt in Deutschland, wo die Hilflosigkeit des Staates gegenüber steigenden Arbeitslosigkeitszahlen und schrumpfenden öffentlichen Finanzen das Nachkriegsmodell der Sozialen Marktwirtschaft zunehmend in Frage stellt, wendet sich mancher Blick zu den britischen Erfahrungen hin.

Ich verbrachte meine Schulzeit im London der 70er Jahre, und meine Erinnerungen sind stark durch die damaligen wirtschaftlichen Schwierigkeiten geprägt. Zeitweise waren die Preise bei jedem Wocheneinkauf höher. Streiks im öffentlichen Dienst führten zu Bergen von stinkenden, rattenverseuchten Müllsäcken an den Straßenecken, zu schulfreien Tagen oder zum Ausfall von Leistungen im Gesundheitswesen. Am stärksten bleiben die Auswirkungen der streikenden Bergarbeiter im Gedächtnis. Während diesen fiel der Strom fast täglich für einige Stunden aus. Den Höhepunkt bildete die sogenannte "three day week". Um die schwindenden Energievorräte zu rationieren, blieben nicht nur die arbeitende Bevölkerung, sondern auch die Schüler zwei Tage in der Woche zu Hause. Als Schulkind habe ich mich darüber gefreut. Als Ökonomin von heute macht mich die Vorstellung der volkswirtschaftlichen Kosten derartiger Maßnahmen fast schwindlig.

Ohne Zweifel hat die Regierung Thatcher diesen Krisen ein Ende gesetzt, hat mit beispielhaften mikroökonomischen Reformen sehr viel zum Positiven verändert. Auch in Bezug auf makroökonomische Bemühungen zur Gesundung der Staatsfinanzen wird ihr eine Vorreiterrolle eingeräumt. Gerade vor dem Hintergrund der jetzigen deutschen Entwicklung ist die Bewunderung, die der Konservativen Regierung hierzulande häufig zuteil wird, durchaus verständlich. Diese Beurteilung kontrastiert jedoch mit einem sehr viel differenzierten Bild im Vereinigten Königreich selbst. Das Urteil über die makroökonomische Politik fällt in der britischen Literatur nicht selten kritisch aus. Zudem werden nun gravierende Umverteilungswirkungen infolge der mikroökonomischen Reformen deutlich, welche deren langfristig positiven Wirkungen für manche Beobachter fraglich erscheinen lassen.

Es lag deshalb nahe, mich im Rahmen meiner Dissertation mit diesen unterschiedlichen Ansichten auseinanderzusetzen. Im Hinblick auf die derzeitigen Reformbemühungen in Europa zur Verwirklichung der Währungsunion erschien es mir spannend und notwendig, die langfristigen Wirkungen der Konservativen Strategie zur Konsolidierung der öffentlichen Haushalte gründlich zu untersuchen und $\mathrm{zu}$ beurteilen. 
Judith Safford - 978-3-631-75199-2

Downloaded from PubFactory at 01/11/2019 07:12:45AM

via free access 


\section{Inhaltsverzeichnis}

\section{PROBLEMSTELLUNG}

ERSTES KAPITEL

\section{ALLGEMEINER TEIL I: AUSWIRKUNGEN DER STAATSVERSCHULDUNG}

I. Schuldenarithmetische Grundzusammenhänge:

Die staatliche Budgetrestriktion 5

1) Grundsätzliches zur Entstehung der Staatsverschuldung 5

2) Die zeitliche Entwicklung der Staatsverschuldung 6

II. Ist Staatsverschuldung irrelevant?

Das Theorem der Staatsschuldneutralität $\quad 8$

1) Die Grundaussage $\quad 8$

2) Prämissen für die Staatsschuldneutralität $\quad 10$

a) Rationale und weitsichtige Haushalte 10

b) Vollkommener intergenerationeller Altruismus 11

c) Vollkommene Kapitalmärkte $\quad 12$

d) Umverteilungseffekte der Finanzierungswahl 13

e) Pauschalsteuern 14

f) Schuldfinanzierung der Zinszahlungen 14

3) Empirische Untersuchungen $\quad 19$

III. „Crowding out“ Effekte der Staatsverschuldung 20

1) Kurzfristiges Crowding Out 21

a) Crowding Out im IS-LM Modell 21

b) Klassisches "Crowding Out" 22

2) „Crowding Out“ unter Berücksichtigung rationaler Erwartungen 23

3) Langfristiges „Crowding-Out“

IV. Auswirkungen der Staatsverschuldung auf die Kapitalbildung 29

1) Der Transferansatz: Die Last der Auslandsverschuldung 30

2) Portfolioeffekte einer staatlichen Schuldaufnahme 30

3) Die Finanzierung der Zinszahlungen 31

4) Die Auswirkungen von Faktorpreisänderungen 33

5) Staatsverschuldung in der offenen Wirtschaft 34

6) Öffentliche Kapitalbildung und Wirtschaftswachstum 35 
V. Wirkungen der Staatsverschuldung auf die Einkommensverteilung 36

1) Intertemporale Wirkungen 36

a) Intertemporale Wirkungen nach der herkömmlichen Schuldentheorie

b) Intertemporale Effekte nach dem , Generational Accounting “ Ansatz 37

2) Intratemporale Verteilungswirkungen $\quad 39$

a) Interpersonelle Verteilungswirkungen im Transferansatz 39

b) Funktionelle Verteilungswirkungen im neoklassischen Ansatz 39

c) Intratemporale Verteilungswirkungen in der heutigen Diskussion $\quad 40$

3) Polit-ökonomische Ansätze zur Verteilung und Staatsverschuldung 41

a) Intergenerationale Umverteilungen 41

b) Intragenerationale Verteilungskonflikte 44

VI. Zusammenfassung des ersten Kapitels 46

$\begin{array}{ll}\text { ZWEITES KAPITEL } & 49\end{array}$

\section{ALLGEMEINER TEIL II: STRATEGIEN ZUR SENKUNG DER} STAATSVERSCHULDUNG

I. Ein Überblick über die Konsolidierungsoptionen 49

1) Finanzierungsquellen im Lichte der staatlichen Budgetbeschränkung 49

2) Die Abgrenzung der Finanzierungsquellen 51

a) Kredit-versus Steuerfinanzierung 51

b) Kredit-versus Geldschöpfungsfinanzierung 52

c) Kreditfinanzierung versus Verkauf von Aktiva 52

II. Strategie I: Der Inflationsmechanimus als

Lösung des Schuldenproblems $\quad 54$

1) Schuldfinanzierung aus der Geldemission $\quad 55$

2) Inflation zur Entwertung des Schuldenstands $\quad 59$

3) Inflationswirkungen auf das Steuer- und Ausgabensystem 61

III. Strategie II: Steuererhöhungen unter Berücksichtigung der Aufkommenselastizität

1) Die Analyse der Laffer-Kurve 63

2) Kollektive Bewegungen des Steuerwiderstands $\quad 69$

3) Grenzen der Besteuerung durch Steuerharmonisierung 71 
IV. Strategie III: Eine Senkung der öffentlichen Ausgaben 75

1) Theorien zur Entwicklung der Staatsausgaben 75

2) Nachfragebedingte Theorien 76

a) Der Staat im Strukturwandel 76

b) Der Staat im Funktionswandel 77

c) Theorien über das Verhalten von Interessengruppen 82

3) Angebotsbedingte Theorien 83

a) Steigende Stückkosten der öffentlich bereitgestellten Güter 84

b) Der Einfluß der Bürokratie $\quad 85$

c) Die Entscheidungsstrukturen und -praktiken der Regierung 87

4) Schlußfolgerungen für die Strategien zur Ausgabensenkungen $\quad 88$

V. Strategie IV: Die Veräußerung von öffentlichem Vermögen 90

VI. Zusammenfassung des zweiten Kapitels $\quad 92$

$\begin{array}{lr}\text { DRITTES KAPITEL } & 95\end{array}$

DIE SCHULDENPOLITIK DER KONSERVATIVEN REGIERUNG 95

I. Historischer Abriß der Staatsverschuldung im Vereinigten Königreich $\quad 95$

II. Aktueller geschichtlicher Hintergrund:

Die britische Wirtschaft nach $1945 \quad 98$

III. Die schuldenpolitische Konzeption der Konservativen Regierung 102

1) Die Konservative Interpretation der Wirtschaftsentwicklung 102

2) Die Konservative Lösung - ,a spoonful of courageous medicine“ 103

3) Die mittelfristige Finanzstrategie 105

4) Die verwendeten Strategien zur Senkung des Budgetdefizits 109

a) Ausgabensenkungen $\quad 110$

b) Die Privatisierungspolitik 111

IV. Die britische Budgetpolitik im Lichte der staatlichen Budgetbeschränkung

1) Die Abgrenzung des Budgetdefizits im Vereinigten Königreich 111

2) Die Bedeutung der Staatsausgaben bei der mittelfristigen Finanzstrategie

3) Die Auswirkungen der Inflation auf die Budgetbeschränkung 113

a) Die Auswirkungen der Inflation auf das Defizitziel 113

b) Der Einfluß der Inflation auf die Staatsausgaben 118 


\section{EINE ANALYSE DER SCHULDENSENKUNG IM VEREINIGTEN KÖNIGREICH}

I. Der öffentliche Kreditbedarf, das reale Finanzierungsdefizit und der Schuldenstand

1) Die Abgrenzung des Finanzierungsdefizits

2) Die Auswirkungen von Wachstum, Inflation und Neuverschuldung auf die Schuldenquote

3) Inflationsbereinigung des Finanzierungsdefizits

4) Auswirkungen der Inflation auf die Steuereinnahmen

II. Die Zusammensetzung des Finanzierungsdefizits und ihre institutionelle Aufteilung

1) Die Finanzierung des staatlichen Budgets

a) Einnahmen aus der Geldemission

127

b) Finanzierung aus der Privatisierungspolitik

c) Der Anteil der Schuldfinanzierung

2) Die Aufteilung der Verschuldung zwischen den öffentlichen Schuldnern

III. Die budgetpolitischen Faktoren der Schuldensenkung 130

1) Der Primärsaldo

2) Der Beitrag der Einnahmen

a) Die Entwicklung der gesamten Einnahmen

b) Steuerreform und Steuereinnahmen

3) Der Beitrag der Staatsausgaben

a) Die Entwicklung der gesamten Ausgaben 139

b) Maßnahmen zur Senkung der Ausgaben 141

c) Die Verteilung der Ausgaben nach Aufgabenbereichen 143

d) Die Verteilung der Ausgaben nach der Verwendungsrechnung 145

e) Die Entwicklung der Transferzahlungen 148

4) Die Zinszahlungen für die Staatsschuld 152

IV. Zusammenfassung des vierten Kapitels 154

FÜNFTES KAPITEL

I. Die Entwicklung der Staatseinnahmen

1) Der Wegfall der Inflation als Lösung des Schuldenproblems 
2) Erlöse aus der Privatisierung 158

3) Steigende Steuereinnahmen 159

II. Die Entwicklung der Staatsausgaben 161

1) Wachsender Druck zu Mehrausgaben 161

2) Exkurs: Der Einfluß der Einkommensverteilung auf die Ausgaben 164

a) Die Entwicklung der Einkommensverteilung seit 1979

b) Folgen der Konservativen Politik auf die Einkommensverteilung 166

3) Steigende Staatsausgaben für Umverteilung 167

III. Die Finanzierung zusätzlicher Staatsausgaben $\quad 170$

1) Marktorientierte Mechanismen $\quad 170$

2) Finanzierung durch Staatsverschuldung 171

$\begin{array}{ll}\text { IV. Ausblick } & 173\end{array}$

$\begin{array}{ll}\text { ANHANG } & 175\end{array}$

I. Crowding Out in IS-LM Modell (1. Kapitel, Abschnitt III) 175

1) Das kurzfristige IS-LM Modell 175

2) Das klassische Modell 176

3) Das langfristige IS-LM Modell 176

II. Zur Berechnung der Entwertung des Schuldenstandes durch die Inflation (2. Kapital, Abschnitt II.2)

III. Berechnung der Veränderung der Schuldenquote, aufgeteilt nach Inflations-, Wachstums- und Neuverschuldungseffekten (4. Kapitel, Abschnitt I.2)

IV. Methodische Anmerkungen zur Berechnung der Inflationsbereinigung des Finanzierungsdefizits (4. Kapitel, Abschnitt I.3)

V. Wichtige Privatisierungen und ihre Erlöse 188

VI. Statistische Grundlage (3. und 4. Kapitel) 189

$\begin{array}{ll}\text { LITERATURVERZEICHNIS } & 203\end{array}$ 


\section{Abbildungsverzeichnis}

Abbildung 1: Die Brutto Staatsverschuldung der G7 Länder ............................... 2

Abbildung 2: Der kurzfristige Effekt einer expansiven Fiskalpolitik ................. 22

Abbildung 3: Kontraktive Wirkung der Defizitpolitik ..................................... 27

Abbildung 4: Instabilität bei expansiver Wirkung der Defizitpolitik................... 27

Abbildung 5: Stabilität bei expansiver Wirkung der Defizitpolitik .................... 28

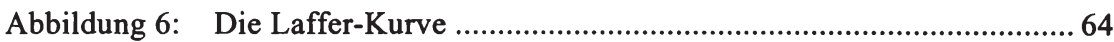

Abbildung 7: Die Laffer-Kurve und die Effizienz des Steuerstaates .................... 68

Abbildung 8: Steigende Staatsausgaben durch Verteilungspolitik.......................79

Abbildung 9: Die Staatsverschuldung im Vereinigten Königreich seit 1700 ...... 96

Abbildung 10: Die Entwicklung der Staatsverschuldung im Vereinigten

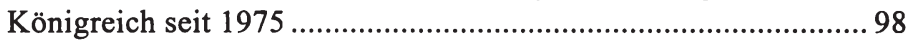

Abbildung 11: Die Ziele der MTFS von 1980/81 bis 1986/87 .......................... 115

Abbildung 12: Die realisierte MTFS: Inflation und der PSBR 1980 bis $1986 \ldots 117$

Abbildung 13: PSBR, Finanzierungsdefizit und Schuldenquote........................ 122

Abbildung 14: Zerlegung der Veränderungen der Schuldenquote 1979-1994 .... 124

Abbildung 15: Inflationsbereinigung des Finanzierungsdefizits....................... 125

Abbildung 16: Quellen der Defizitfinanzierung im Vereinigten Königreich ...... 127

Abbildung 17: Die Finanzierungsdefizite der öffentlichen Institutionen ............ 129

Abbildung 18: Der Primärsaldo 1979 bis 1994 ................................................. 132

Abbildung 19: Die Entwicklung der Staatseinnahmen 1974-1994 ..................... 134 
Abbildung 20: Die Zusammensetzung der britischen

Steuereinnahmen 1979-1994

Abbildung 21: Die Entwicklung der Staatsausgaben 1974-1994....................... 139

Abbildung 22: Die britischen Staatsausgaben nach Aufgabenbereichen ............ 143

Abbildung 23: Die Entwicklung der Ausgaben nach investiven und konsumtiven Bereichen

Abbildung 24: Die Staatsausgaben nach der Verwendungsrechnung

Abbildung 25: Die Entwicklung der laufenden Transferzahlungen und der Subventionen

Abbildung 26: Die Entwicklung der persönlichen Transferzahlungen 150

Abbildung 27: Die Brutto-Zinszahlung auf die Staatsverschuldung 153

Abbildung 28: Der Deckungsgrad der Realzinslast durch den Primärüberschuß 1979 bis 1994

Abbildung 29: Anteile der Einkommensverteilung 164

Abbildung 30: Durchschnittliche Einkommensveränderungen zwischen 1979 und 1988/89 nach Einkommensgruppen. 166

Abbildung 31: Dynamische Stabilität im vermögenserweiteten IS-LM Modell

Abbildung 32: Vergleich zwischen berechneter und tatsächlicher

Verschuldungsquote. 185 
XVI

\section{Tabellenverzeichnis}

Tabelle 1: Ausgewählte Steuersätze in der EU (12) 1994...................................... 73

Tabelle 2: Ökonomische Entwicklung des Vereinigten Königreichs und der OECD 1948-79 (Zunahme per annum in \%).......................... 100

Tabelle 3: Die MTFS, Geldmengenwachstum und der PSBR .............................. 114

Tabelle 4: Inflation und der PSBR 1980-1994............................................. 116

Tabelle 5: Die wichtigsten britischen Steuersätze ............................................ 137 


\section{Abkürzungsverzeichnis}

\begin{tabular}{ll} 
BIP & Bruttoinlandsprodukt \\
BoE & Bank of England \\
BoEQB & Bank of England Quarterly Bulletin \\
BSP & Bruttosozialprodukt \\
CSO & Central Statistical Office \\
EU & Europäische Union \\
HMSO & Her Majesty's Stationary Office \\
IMF/IWF & Internationaler Währungsfonds \\
MTFS & $\begin{array}{l}\text { Medium-term financial strategy } \\
\text { (mittelfristige Finanzstrategie) }\end{array}$ \\
NECD & National Economic Development Council \\
NCT & New Control Total \\
OECD & Organization for Economic Co-operation and Development \\
OEEC & Organization for European Economic Co-operation \\
PSBR & Public Sector Borrowing Requirement \\
PSDR & Public Sector Debt Repayment \\
PT & Planning Total \\
UK & United Kingdom (Vereinigtes Königreich) \\
ZS & Zentralstaat \\
\hline
\end{tabular}


Judith Safford - 978-3-631-75199-2

Downloaded from PubFactory at 01/11/2019 07:12:45AM

via free access 


\section{PROBLEMSTELLUNG}

Staatsverschuldung und öffentliche Defizite sind ein hoch aktuelles Thema geworden. Wenn die Inflationsbekämpfung die makroökonomische Herausforderung der 80er Jahre darstellte, dürfte sich die Konsolidierung der Staatsfinanzen als die größte Aufgabe für die Regierungen der $90 \mathrm{er}$ Jahre erweisen. Nach verbreiteter Meinung ${ }^{1}$ stellt die Höhe der Staatsverschuldung in vielen Ländern eine unvertretbare Belastung für die zukünftigen Generationen dar; ihr Anstieg ist die Hauptursache für das gegenwärtig hohe Niveau des Realzinses, sowie für die Währungsturbulenzen, in denen sich die Währungen hochverschuldeter Länder, wie z. B. der U.S.A. oder von Italien, abwerten. Ferner wächst in vielen sogenannten Entwicklungsländern sowie einigen Industrieländern die Besorgnis, daß die Last der Staatsverschuldung unablässig wachsen wird. Die betroffenen Regierungen werden dazu gezwungen, durch Geldschöpfung oder Zahlungsverweigerung die steigenden Tilgungs- und Zinslasten zu stabilisieren. Schließlich wird in den Industrieländern die Überalterung der Bevölkerung in den kommenden Jahren zusätzliche Ausgaben für Pensionszahlungen und Gesundheit mit sich bringen, was die Belastung der Staatsfinanzen noch weiter verschärfen wird.

So ist die Kontrolle und die Senkung des öffentlichen Defizits in vielen Ländern zum Eckstein der makroökonomischen Politikgestaltung geworden. Der traditionelle Ansatz, Budgetdefizite zur zyklischen Stabilisierung einzusetzen, wurde dadurch stark in den Hintergrund gedrängt.

Ein Bedeutungsgewinn für fiskalpolitische Orthodoxie und solide öffentliche Finanzen - das Erste als Vorbedingung für das Zweite - läßt sich seit Anfang der 80er Jahre als internationales Phänomen feststellen. In den Vereinigten Staaten schlug sich der fiskalpolitische Wandel in dem 1985 erlassenen Gramm-RudmanHollings-Gesetz nieder, das ein ausgeglichenes Budget bis 1991 vorsah. Fiskalpolitische Orthodoxie ist ferner zu einem zentralen Baustein der Konditionalität und Leihbedingungen des Internationalen Währungsfonds und der Weltbank geworden. In Deutschland intensivierte sich die Kritik und öffentliche Besorgnis über die Staatsfinanzen mit den steigenden Kosten der Deutschen Wiedervereinigung. Schließlich enthalten die Konvergenzkriterien des Maastrichter Vertrags der Europäischen Union von 1992 Bedingungen bezüglich der Höhe des Budgetdefizits und der Staatsverschuldung im Verhältnis zum Bruttoinlandsprodukt. Damit soll den Mitgliedsländern eine Budgetdisziplin aufgezwungen werden. 
Es besteht zwar Einigkeit über die schuldenpolitische Zielsetzung. Die tatsächlichen Verhältnisse sehen jedoch anders aus: Im Oktober 1997 scheint es, daß nur eine flexible Handhabung der Kriterien und das unerwartet starke Wirtschaftswachstum den meisten EU-Mitgliedern die Erfüllung der beiden Maastrichter Konvergenzkriterien für die Staatsfinanzen ermöglichen wird. ${ }^{2}$ In den nicht-europäischen Industrieländern ist es mit der Staatsverschuldung auch nicht besser bestellt. Die Abbildung 1 zeigt die Entwicklung der Schuldenquote in den G7Ländern von 1978 bis 1994.

\section{Abbildung 1: Die Brutto Staatsverschuldung der G7 Länder}

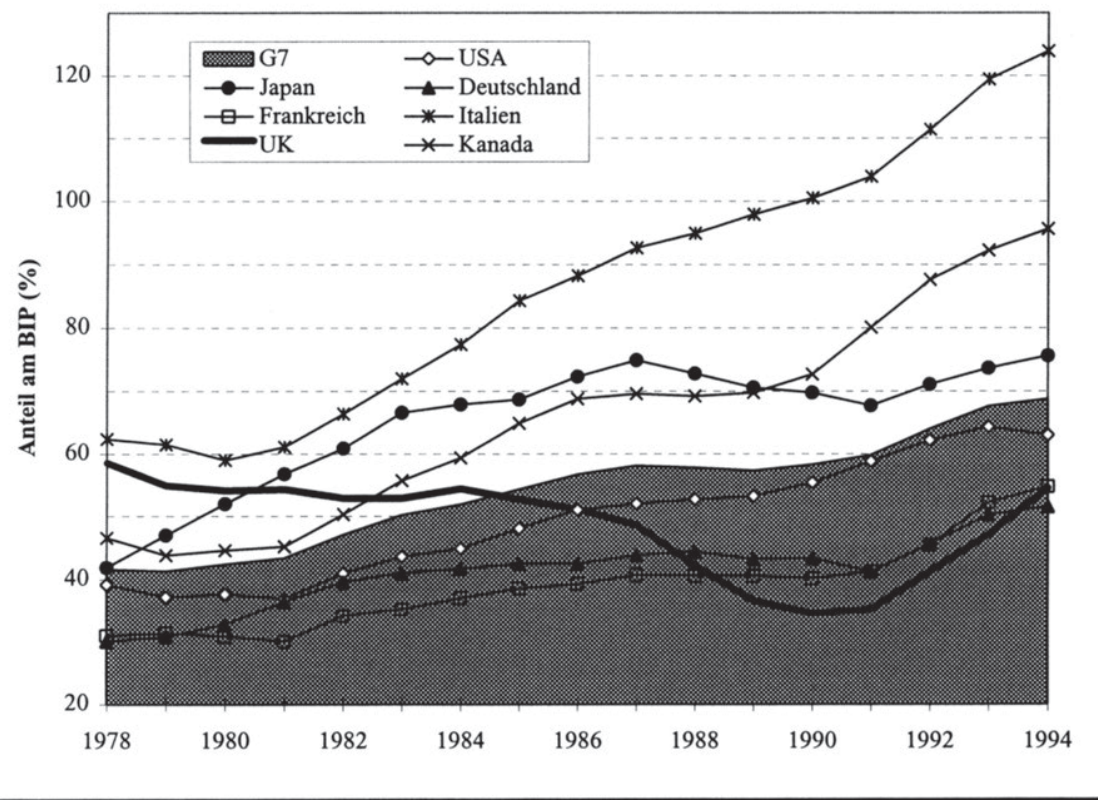

Quelle: OECD (1995) Wirtschaftsausblick, Tab. 34

Die durchschnittliche Schuldenquote stieg zwischen 1978 und 1994 von 41,6\% auf 68,8\%. Den stärksten Anstieg erfuhr Italien, dessen Schuldenquote sich um über $60 \%$ erhöht hat. Den geringsten Quotenansteig verzeichnete Deutschland mit $21 \%$. Als einzigem unter all diesen Ländern ist es dem Vereinigten Königreich gelungen, in dieser Zeit seine Schuldenquote zu senken. Sie fiel zwischen 1978 und 1990 um $24 \%$ von 58,6 auf $34,6 \%$. Danach allerdings stieg die Quote bis 1994 rapide wieder auf $54,5 \%$ an.

${ }^{2}$ Economist, 18.10.97, S. 36. 
Dieser auf den ersten Blick einzigartige Erfolg ruft nach einer näheren Betrachtung. Er fiel eng mit einem Regierungswechsel zusammen, bei dem im Mai 1979 die Konservative Partei die Macht mit einer absoluten Mehrheit in beiden Parlamentshäusern von der Labour Partei übernahm. Der Schuldenabbau war eines der zentralen Politikzielen der Konservativen Regierung. Im Rahmen dieser Arbeit soll geklärt werden, wie diese Konsolidierung erreicht wurde. Aus den Ergebnissen soll dann auf die Konsequenzen für die zukünftige Schuldenentwicklung geschlossen werden.

Im ersten Teil der Arbeit wird mit einer Erörterung der grundlegenden Zusammenhänge und Theorien zur Staatsverschuldung das Fundament gelegt. Er beginnt mit einer kurzen Darstellung der schuldenarithmetischen Grundzusammenhänge. Anschließend wird die ökonomische Relevanz von öffentlicher Verschuldung und von Budgetdefiziten erörtert. Nach einer Diskussion der Bedingungen für Schuldenneutralität werden die Wirkungen der Staatsverschuldung aus der Sicht der keynesianischen und neoklassischen Theorien dargestellt. Das Kapitel schließt mit einer Darstellung des Nexus zwischen Einkommensverteilung und Staatsverschuldung, weil dieser Zusammenhang ein Schwerpunkt der Arbeit sein wird. Das zweite Kapitel untersucht, ebenfalls auf theoretischer Ebene, die möglichen Strategien zur Senkung der Staatsverschuldung. Dabei wird jedoch zur Illustration hie und da schon ganz konkret auf die Umstände und Erfahrungen im Vereinigten Königreich Bezug genommen. Die Bestandteile der Budgetbeschränkung werden im einzelnen daraufhin untersucht, inwiefern und unter welchen Bedingungen sie einen Beitrag zur Schuldenstabilisierung leisten können.

Das dritte Kapitel geht zu den realen Erfahrungen im Vereinigten Königreich über. Es stellt erstens die Konservative Schuldenpolitik vor dem Hintergrund der Nachkriegsgeschichte und der Konservativen wirtschaftspolitischen Konzeption dar. Zweitens folgt eine detaillierte Analyse der britischen ${ }^{3}$ Budgetpolitik, ihrer Ziele und wie diese erreicht werden sollten. Im Anschluß daran folgt im vierten Kapitel die empirische Analyse der Schuldenpolitik. Es wird gezeigt, wie die Veränderungen der einzelnen Komponenten der staatlichen Budgetrestriktion zum Schuldenabbau beigetragen haben. Im letzten Kapitel werden Schlüsse über den Erfolg und die vermutliche Dauerhaftigkeit der Konsolidierung gezogen. Ein besonderes Augenmerk gilt dabei dem Zusammenhang zwischen der Entwicklung der Einkommensverteilung einerseits und dem Erfolg der Konsolidierungspolitik andererseits. Insbesondere wird untersucht, inwiefern die Einkommensverteilung die Neigung

${ }^{3}$ Das Vereinigte Königreich besteht aus England, Nordirland, Schottland und Wales. Dies ist von „Großbritannien“ zu unterscheiden, zu dem Nordirland nicht zählt. In dieser Abhandlung wird das Adjektiv „britisch“ für das Vereinigte Königreich verwendet und bezieht sich somit auf alle vier Länder. 
zur Kreditfinanzierung und somit die zukünftige Schuldenentwicklung beeinflussen könnte. 


\section{Erstes Kapitel}

\section{ALLGEMEINER TEIL I: AUSWIRKUNGEN DER STAATSVERSCHULDUNG}

Stellen Budgetdefizite und steigende Staatsverschuldung wirklich ein ökonomisches Problem dar? Bedeutete die Schuldentilgung der 80er Jahre unter der Konservativen Regierung einen Wohlstandsgewinn für die britische Wirtschaft? $\mathrm{Zu}$ den Auswirkungen der Staatsverschuldung lassen sich zumindest drei sehr verschiedenartige Ansichten unterscheiden: Aus den Keynesianischen Wirkungszusammenhängen lassen sich positive kurzfristige Beschäftigungs- und Einkommenswirkungen ableiten. ${ }^{4}$ Dagegen gelangt die neoklassische Analyse aufgrund langfristiger Effekte auf die gesamtwirtschaftliche Kapitalbildung zu einem negativen Urteil. ${ }^{5}$ Schließlich bestreitet eine dritte Lehrmeinung jegliche realwirtschaftlichen Konsequenzen der Staatsverschuldung. ${ }^{6}$ Nach diesem Theorem der Schuldneutralität wäre die wirtschaftliche und politische Diskussion über die Staatsverschuldung im Grunde genommen überflüssig. Deshalb wird diese These nach einer kurzen Darstellung der schuldenarithmetischen Grundzusammenhänge zuerst dargestellt.

\section{Schuldenarithmetische Grundzusammenhänge: Die staatliche Budgetrestriktion}

\section{1) Grundsätzliches zur Entstehung der Staatsverschuldung}

Im folgenden sollen nun einige grundlegende Begriffe und Zusammenhänge erläutert werden. Unter Staatsverschuldung bzw. der Staatsschuld wird eine Kreditaufnahme des Staates verstanden, bei der Dritte dem Staat Ressourcen in Form von Gütern oder Geld zur Verfügung stellen. Als Gegenwert verpflichtet sich der Staat zu einer Rückzahlung der Ressourcen in der Zukunft. ${ }^{7}$ Um sich darüber klar zu werden, wie die Staatsverschuldung zustande kommt, wie ihre dynamische Ent-

\footnotetext{
${ }^{4}$ Vgl. z. B. Blinder und Solow $(1973,1974)$.

${ }^{5}$ Siehe z. B. Diamond (1965).

${ }^{6}$ Hauptvertreter dieser Ansicht ist Barro (1974, 1989).

${ }^{7}$ Vgl. Andel (1990), S. 359.
} 
wicklung bestimmt wird und wie sie sich von anderen Finanzierungsmöglichkeiten unterscheidet, läßt sich die staatliche Budgetrestriktion heranziehen. In ihrer einfachsten Form besagt diese, daß sämtliche staatlichen Ausgaben durch staatliche Einnahmen abgedeckt sein müssen. Die einzelnen Einnahme- und Ausgabenposten werden in Abhängigkeit von der Modellkonstruktion sowie dem Ziel der Untersuchung unterschiedlich stark untergliedert. ${ }^{8}$ In der Regel werden als staatliche Ausgaben die Zins- und andere Transferzahlungen, sowie die Bereitstellung von Gütern und Dienstleistungen erfaßt. Einnahmen erfolgen durch die Erhebung von Steuern, Schuldaufnahme beim Privatsektor oder im Ausland, die Veräußerung von öffentlichem Vermögen oder durch eine Erhöhung der Geldemission.

Eine einfache Formulierung der staatlichen Budgetrestriktion läßt sich dann wie folgt darstellen:

$$
P_{t} G_{t}+T r_{t}-T_{t}+\left(1+i_{t-1}\right) B_{t-1}-\left(M_{t}-M_{t-1}\right)-\left(A_{t}-A_{t-1}\right)=B_{t}
$$

$B_{t}$ ist der nominale Bruttoschuldenstand, der sich aus vier Hauptkomponenten zusammensetzt. $G_{t}$ sind die realen Staatsausgaben und $P_{t}$ ist das Preisniveau, $T r_{t}$ sind die Transfers außer Zinszahlungen auf der Staatsschuld und $T_{t}$ sind die Steuereinnahmen und Sozialversicherungsbeiträge. Die Differenz zwischen diesen Einnahmen und Ausgaben bildet die erste Komponente, den sogenannten Primärsaldo. Die zweite Komponente sind die Zins- und Tilgungszahlungen der vergangenen Verschuldung $\left(1+\mathrm{i}_{\mathrm{t}-1}\right) \mathrm{B}_{\mathrm{t}-1}$. $\mathrm{B}_{\mathrm{t}-1}$ bezeichnet den Bruttoschuldenstand der Vorperiode $\mathrm{t}-1$. In Periode $t$ wird $B_{t-1}$ verzinst zum Nominalzins $i_{t-1}$ und annahmegemä $\beta$ vollständig getilgt. Der Zinssatz $i_{t-1}$ wurde in Periode t- 1 zwischen dem Staat als Kreditnehmer und den privaten Haushalten als Kreditgeber vereinbart. ${ }^{9}$ Er setzt sich aus dem Realzins $r$ und der für Periode $t$ erwarteten Inflationsrate $\pi_{t}{ }^{e}$ zusammen. Die dritte Komponente umfaßt die Veränderung der Geldbasis $\left(M_{t}-M_{t-1}\right)$, bzw. die Geldschöpfung zur Staatsfinanzierung unter der Voraussetzung, daß die Notenbank und die Zentralregierung institutionell vereint sind. Die vierte Komponente ist die Veränderung des öffentlichen Vermögensbestandes $\left(A_{t}-A_{t-1}\right)$.

\section{2) Die zeitliche Entwicklung der Staatsverschuldung}

Um die dynamische Entwicklung der Budgetbeschränkung im Zeitverlauf näher zu untersuchen, ist es zweckmäßig, diese Größen im Verhältnis zum Bruttoinland-

\footnotetext{
${ }^{8}$ Siehe z. B. Christ (1979) S. 526.

${ }^{9}$ Diese Annahme bedeutet eine wesentliche Vereinfachung der Analyse. Geldpolitische Fragen der Fristenstruktur der Staatsverschuldung werden ausgesshlpssenfford - 978-3-631-75199-2
} 
sprodukt $\left(Y_{n}=Y P\right)$ auszudrücken. Wir definieren die Schuldenquote als $b=B / Y_{n}$. Die Entwicklung der Schuldenquote ergibt sich dann als

$$
\dot{\mathrm{b}}=\frac{\dot{\mathrm{B}}}{\mathrm{Y}_{\mathrm{n}}}-\mathrm{b} \frac{\dot{\mathrm{Y}}_{\mathrm{n}}}{\mathrm{Y}_{\mathrm{n}}^{2}}
$$

oder umformuliert

$$
\text { (2) } \dot{\mathrm{B}}=\dot{\mathrm{b}} \mathrm{Y}_{\mathrm{n}}+\mathrm{B} \frac{\dot{\mathrm{Y}}_{\mathrm{n}}}{\mathrm{Y}_{\mathrm{n}}}
$$

durch Einsetzen von (2) in (1) ergibt sich

$$
\text { (3) } g+\operatorname{tr}-\mathrm{t}+\mathrm{ib}-\dot{\mathrm{m}}-\dot{\mathrm{a}}=\dot{\mathrm{b}}+\mathrm{b} \frac{\dot{\mathrm{Y}}_{\mathrm{n}}}{\mathrm{Y}_{\mathrm{n}}}
$$

wobei die Kleinbuchstaben die jeweiligen Einkommensquoten darstellen $\left(\mathrm{X} / \mathrm{Y}_{\mathrm{n}}=\mathrm{x}\right)$. Durch eine weitere Umformulierung von (3) erhalten wir einen Ausdruck für die Entwicklung der Schuldenquote in Abhängigkeit von der Budgetbeschränkung: ${ }^{10}$

$$
\text { 4) }(\mathrm{g}+\mathrm{tr}-\mathrm{t})-\dot{\mathrm{m}}-\dot{\mathrm{a}}+\mathrm{b}(\mathrm{i}-\phi)=\dot{\mathrm{b}}
$$

wobei $\phi$ die Wachstumsrate des nominalen Einkommens darstellt.

Anhand der Gleichung (4) lassen sich die verschiedenen Alternativen zur Stabilisierung oder zum Abbau der Verschuldung untersuchen. Unter Stabilisierung wird eine Politik verstanden, welche die Entwicklung der Verschuldungsquote zum Stillstand bringt (das heißt, es gilt: $\dot{b}=0$ ). Ein Schuldenabbau bedeutet, da $\beta$ die Verschuldungsquote abnimmt (das heißt, es gilt: $\dot{b}<0$ ). Die Verzinsung (i) auf den Schuldenstand (b) wirkt sich positiv auf die Verschuldungsquote $(\dot{b}>0)$ aus. Dagegen nimmt sie entsprechend der Wachstumsrate des Bruttoinlandprodukts $(\phi)$ ab. Liegt der Zinssatz über der Wachstumsrate, dann nimmt der Schuldenstand stetig zu. ${ }^{11}$ Die dynamische Verschuldungszunahme kann nur durch einen Primärüber$\operatorname{schuß}(\mathrm{g}+\mathrm{tr}-\mathrm{t}<0)$, den Verkauf von öffentlichen Aktiva $(\dot{a}>0)$ oder eine Erhöhung der Geldmenge $(\dot{m}>0)$ aufgehalten werden.

${ }^{10}$ Die Verschuldungsdynamik wird in ähnlicher Weise z. B. bei Blanchard (1990), S. 13ff. abgeleitet.

11 Der dynamische Zusammenhang zwischen Zins und Wachstumsrate wurde zuerst von Domar (1946) aufgezeigt. Er wird im Abschnitt IV.3 ausführlich erläutert. 
Die Theorie der optimalen Finanzpolitik besagt, daß eine rationale Regierung alle ihr offenen Finanzierungsquellen ausschöpfen wird, bis ihre jeweiligen marginalen Kosten sich ausgleichen. ${ }^{12}$ Die vorliegende Arbeit beschäftigt sich nicht mit derartigen normativen Überlegungen, sondern mit der Frage, welche dieser Variablen in welchem Ausmaß die Konservative Regierung zur Schuldensenkung tatsächlich herangezogen hat, wie diese Auswahl zu erklären ist und welche Konsequenzen für die zukünftige Schuldenentwicklung zu erwarten sind.

Die Wachstumsrate des nominalen Einkommens $(\phi)$ ließe sich durch einen Anstieg der Inflationsrate oder der realen Wachstumsrate erhöhen. Dabei wäre zu klären, inwiefern die Regierung die Inflationsrate oder das reale Wachstum endogenisieren kann. Dagegen liegen die Variablen des Primärsaldos (g-t) prinzipiell im Einflußbereich der Regierung. Einnahmen aus der Veräußerung öffentlichen Vermögens (a) können zur Stabilisierung der Verschuldung herangezogen werden. Auch die Verweigerung der Zinszahlungen (ib) ist eine Politikoption, die zumindest für die Auslandsverschuldung Anwendung finden kann. Schließlich stellt die Geldschöpfungskomponente (m) der Budgetbeschränkung ebenfalls eine mögliche Budgetfinanzierungsquelle dar.

\section{Ist Staatsverschuldung irrelevant?: Das Theorem der Staats- schuldneutralität}

\section{1) Die Grundaussage}

Eine staatliche Kreditaufnahme stellt nichts anderes als eine zeitlich hinausgeschobene Besteuerung dar, wenn zu ihrer Verzinsung und Tilgung Steuern erhoben werden müssen. Demnach sollte es einem rationalen Individuum gleichgültig sein, ob es heute $£ 1$ Steuern bezahlt oder morgen $£ 1$ zuzüglich Zinsen. Bezahlt der Staat seine Ausgaben durch die intertemporale Substitution von Steuern mit einer staatlichen Schuldaufnahme, wird die Äquivalenz dieser zwei Finanzierungsarten von rationalen Steuerzahlern durchschaut. Sie antizipieren eine zukünftige Steuererhöhung für Tilgungs- und Zinszahlungen der heute aufgenommenen Schuld und bilden sofort entsprechende Ersparnisse. Dieses Verhalten führt zu einer Art Say'schem Gesetz der Staatsverschuldung: Die Nachfrage nach Staatsschuldpapieren paßt sich dem erhöhten Angebot an. Unter diesen Bedingungen hat die staatliche Schuldenpolitik keinen Einfluß auf die Budgetbeschränkung der Steuerzahler, und sie vermag rationale Konsumentscheidungen nicht zu beeinflussen. Die zwin-

12 Siehe z. B. Fischer (1982) und Grilli (1989). 
gende Folge ist, daß Steuererhebung und staatliche Kreditaufnahme in ihren ökonomischen Wirkungen vollkommen äquivalent sind. Der Finanzierungmodus von Staatsausgaben hat keinerlei reale Auswirkungen, insbesondere bleibt der Zinssatz unverändert.

Ricardo erkannte vermutlich als erster Ökonom diese theoretische Möglichkeit, ${ }^{13}$ wonach dieser Zusammenhang auch als Ricardianisches Äquivalenztheorem benannt wurde. ${ }^{14} \mathrm{Er}$ lehnte jedoch seine realpolitische Relevanz aus zwei Gründen ab: Erstens könnten rationale Steuerzahler die zukünftige Steuerlast durch Emigration vermeiden, zweitens verhalten sich viele Steuerzahler nicht rational und übersehen die zukünftige Last. Das bedeutet, daß eine staatliche Schuldaufnahme nicht als intertemporale Steuerverschiebung, sondern als Netto-Vermögenszunahme der privatwirtschaftlichen Gläubiger wahrgenommen wird. ${ }^{15}$ Gerade in der neoklassischen Wirkungsanalyse wird angenommen, daß der Planungshorizont der Steuerzahler wegen ihres Ablebens endlich ist (was hier analytisch gleichbedeutend mit Emigration ist) und die zukünftigen Auswirkungen staatlicher Kreditaufnahmen sie deswegen nicht mehr kümmern. Voraussetzung für die Entfaltung des Keynesianischen Einkommensmultiplikators ist ein gegenwärtiger Konsumanstieg, der durch positive Einkommens- und Vermögenseffekte ausgelöst wird. ${ }^{16}$ Diese expansive Wirkung würde häufig auf die scheinbare Täuschung irrationaler Steuerzahler zurückgeführt, welche die zukünftige Last übersehen. Ricardos Einwände gegen die Gültigkeit des Äquivalenztheorems sind (zumindest implizit) als zentrale Annahmen der beiden oben erwähnten Schuldentheorien wiederzufinden.

In einem Modell überlappender Generationen mit rationalen Erwartungen untersuchte Barro (1974), unter welchen Bedingungen und Verhaltensannahmen die Äquivalenz von Schuld- und Steuerfinanzierung entgegen Ricardos Erwartungen dennoch Gültigkeit hat. Er prüfte zuerst den Einwand gegen das Äquivalenztheorem, daß eine schuldfinanzierte Steuersenkung zumindest diejenigen Steuerzahler zu erhöhtem Konsum bewegt, welche der zukünftigen Steuererhöhung durch den Tod (bzw. die Emigration) ausweichen können. Barro (1974) postulierte, daß Steuerzahler sich gegenüber ihren Nachfolgern altruistisch verhielten. Sie berücksichtigten das Nutzenniveau ihrer Kinder in ihrer eigenen Nutzenfunktion und dehnten folglich ihren Planungshorizont über ihre eigene Lebensdauer auf die ihrer Nachfolger - im Extremfall bis ins Unendliche - hinaus. Unter diesen Bedingungen werden sie durch zusätzliches Sparen Vorkehrungen gegen die voraussehbare Steuer-

\footnotetext{
${ }^{13}$ Ricardo [(1821) 1951], Vol. IV, S. 247f.

${ }^{14}$ Der Name wurde zuerst von Buchanan (1976), S. 337 verwendet.

${ }^{15}$ In der Regel werden reale Auswirkungen der staatlichen Kreditaufnahme mit ihrer Wahrnehmung als Realvermögen gleichgesetzt. Diese Annahme gilt auch für die meisten Szenarien, siehe jedoch Carmichael (1979), S. 105ff. für eine Beweisführung, daß dies nicht immer gelten muß.

${ }^{16}$ Siehe z. B. Blinder und Solow (1973), S. 325. 
erhöhung treffen, welche für Zinszahlungen (und gegebenfalls Tilgungen) vergangener staatlicher Kreditaufnahmen benötigt werden. Im Todesfalle werden diese Ersparnisse an ihre Nachfolger vererbt. Für rationale, altruistische Steuerzahler ist es eben gleichgültig, ob sie oder ihre Nachfolger die Steuererhöhungen zahlen. Ihr Planungshorizont reicht in die Zukunft bis zur Schuldentilgung, und sie sind nicht bestrebt, auf Kosten ihrer Nachfolger der Last zu entrinnen.

In einem zweiten Modell griff Barro (1974) die von Ricardo unberücksichtigte Tatsache auf, daß nicht nur die Laufzeit der Schuld im Verhältnis zum Zeithorizont der Steuerzahler, sondern auch ihre Konditionen die Äquivalenzaussage bedingen. Mit anderen Worten, die Steuerzahler müssen die Staatsschuldneutralität nicht nur bewirken wollen, sondern sie auch bewirken können. Dafür setzt er vollkommene Kapitalmärkte voraus. Die Haushalte verwenden eine Steuersenkung vollständig zum Kauf staatlicher Wertpapiere.

Aus Barros (1974) ursprünglicher Diskussion und der Vielzahl der darauffolgenden Beiträge zum Schuldenneutralitätstheorem hat sich ein theoretischer Referenzrahmen gebildet, in dem die Bedingungen für die Staatsschuldneutralität offengelegt werden können. Das Barro-Ricardianische Äquivalenztheorem läßt sich dann als eine Art Nullhypothese interpretieren. Anhand von Variationen ihrer Prämissen können Quellen der Nichtneutralität aufgedeckt und ihre Konsequenzen untersucht werden. ${ }^{17}$ Aus der Diskussion haben sich eine Reihe von Bedingungen für das Äquivalenzergebnis herausgestellt. Stichwortartig sind dies: a) rationale und weitsichtige Haushalte; b) vollkommener intergenerationeller Altruismus; c) vollkommene Kapitalmärkte; d) keine Verteilungseffekte der Finanzierungswahl; e) ein Pauschalsteuersystem und $f$ ) keine Alternative zur Steuerfinanzierung für die Zinszahlungen der Staatsschuld. Im folgenden sollen diese Prämissen diskutiert werden.

\section{2) Prämissen für die Staatsschuldneutralität}

\section{a) Rationale und weitsichtige Haushalte}

Das Äquivalenzergebnis setzt voraus, daß die Haushalte sowohl rational als auch weitsichtig in bezug auf ihr eigenes Einkommen und die zukünftige Last der Ver-

${ }^{17}$ Das Theorem läßt sich auch als eine empirische falsifizierbare Aussage über die Auswirkungen der Staatsverschuldung untersuchen. Eine Zusammenfassung und ausführliche Diskussion der theoretischen und empirischen Arbeiten zur Schuldenneutralität geben z. B. Bernheim (1987), sowie Leiderman und Blejer (1988). Im deutschsprachigem Raum sifhe Huber (1990) $S_{-7} 5^{3}$-d 991 - 2 
schuldung reagieren. Auch wenn die Hypothese der rationalen Erwartungen eine sehr wichtige Rolle in der ökonomischen Analyse eingenommen hat, ist im Auge $\mathrm{zu}$ behalten, daß alternative Verhaltenshypothesen zu anderen nicht-neutralen Ergebnissen führen können. Einige hiervon, sowie die damit verbundenen Schwierigkeiten, werden unter $\mathrm{f}$ ) ausführlicher erörtert.

\section{b) Vollkommener intergenerationeller Altruismus}

Barro unterstellt, daß eine unendliche altruistische Verbindung die Generationen ewig verbindet. Er legt eine Nutzenfunktion zugrunde, in der das Nutzenniveau der (nutzenmaximierenden) Kinder in das der Eltern eingeht. Sie hat z. B. die Form:

$$
U_{t}=U\left(c_{t}\right)+\frac{1}{1+\rho} U_{t+1}
$$

wobei c der Konsum und $\rho$ der interpersonelle Diskontfaktor ist. Da in $U_{t+1}$ das Nutzenniveau der folgenden Generation $U_{t+2}$, in dieses wiederum $U_{t+3}$ eingeht, u.s.w., entsteht für ein Individuum trotz seiner endlichen Lebensdauer ein unendlicher Planungshorizont. Das Nutzenniveau des Individuums schließt das Nutzenniveau aller zukünftigen Generationen mit ein.

Diese Formulierung hat zwei theoretische Komponenten, die zu diskutieren sind. Erstens müssen die intergenerationellen Transfers allgegenwärtig und universal sein. Nach Barro handelt eine „dynastische Familie“ wie ein repräsentatives Individuum mit einem unendlichen Zeithorizont. Offensichtlich wird diese altruistische Generationskette unterbrochen, wenn Individuen kinderlos oder indifferent gegenüber dem Nutzen ihrer Nachfolger sind, weil sie den Nutzen $U_{t+1}$ gar nicht berücksichtigen werden. Es gibt auch sogenannte Randlösungen, bei denen zwar ein Vererbungsmotiv besteht, aber trotzdem nicht vererbt wird. In einer wachsenden Wirtschaft $z$. B. ist zu erwarten, daß aufgrund von Produktivitätswachstum die nachfolgende Generation ohnehin besser gestellt sein wird als ihre Eltern. Ist der abdiskontierte Grenznutzen der Vererbung bei positiven Erbschaften kleiner als der eigene Grenznutzen des Konsums, dann wird unter Ausschluß negativer Erbschaften $^{18}$ nichts vererbt. Um das Äquivalenzergebnis aufrechtzuerhalten, müßten fehlende Erbschaften durch kompensierende Transfers von anderen Familien geleistet

18 Ein älteres Individuum bekommt keinen Kredit, weil es die Zins- und Tilgungszahlungen nicht mehr selbst leisten wird und sich nicht gegen das Einkommen seiner Nachkommen verschulden kann. Vgl. Michaelis (1989), S. 103. 
werden. Geschieht dies nicht vollständig, dann hat eine Defizitfinanzierung reale Wirkungen. ${ }^{19}$

Zweitens hängt das Neutralitätsergebnis vom Altruismus als Vererbungsmotiv ab. Die erbende Generation erhält die Erbschaft unabhängig vom eigenen Verhalten. Diese Annahme ist unter Berücksichtigung der Komplexität und Vielschichtigkeit von familiären Beziehungen sehr restriktiv. ${ }^{20}$ Spielen andere Motive eine Rolle bei der Vererbung, dann wird die Erbschaft der zukünftigen Steuerlast wahrscheinlich nicht genau entsprechen: Das Äquivalenztheorem gilt dann nicht. Bernheim, Schleifer und Summers (1985) unterstellen z. B. ein strategisches Verhaltensmotiv, bei dem eine Erbschaft als Instrument eingesetzt wird, um ein gewünschtes Verhalten der Erben zu bewirken. Der Nutzen der Nachfolger geht nicht in die Nutzenfunktion ein, sondern der Nutzen der Erbschaft als strategisches Mittel. Die Nutzenfunktion hat dann z. B. die Form:

$$
\mathrm{U}_{\mathrm{t}}=\mathrm{U}\left(\mathrm{c}_{\mathrm{t}} ; \mathrm{q}_{\mathrm{t}}\right)
$$

wobei q die Erbschaft und $\mathrm{c}$ der Konsum sind. Beispielsweise können Erbschaften in Aussicht gestellt werden, um sich von den Erben die notwendige Zuwendung und Hilfeleistung im Pflegefall abzusichern. Schließlich können Unsicherheit über die Lebensdauer [Blanchard (1985); Cukierman (1986)] oder hinsichtlich des Lebenseinkommens [Buiter und Tobin (1981)] das Vererbungsmotiv beeinflussen oder infolgedessen zu sogenannten „Zufallserbschaften“ führen.

\section{c) Vollkommene Kapitalmärkte}

Die Prämisse der Kapitalmarktvollkommenheit in Barros zweitem Ansatz knüpft nicht an das Verhaltensmotiv, sondern an Verhaltensmöglichkeiten an, welche eindeutige Bedingungen voraussetzen. Kapitalmarktvollkommenheit bedeutet, da $\beta$ es keine infolge schlechter Bonität liquiditätsbeschränkten Steuerzahler gibt, für die eine staatliche Kreditaufnahme neue Konsummöglichkeiten eröffnet. Wenn dagegen wegen privater Kreditrationierungen nicht alle Haushalte Kredite bekommen, oder wenn sich die Kreditkonditionen für die Haushalte und den Staat unterscheiden, dann kann eine staatliche Kreditaufnahme für bestimmte Individuen ansonsten unerreichbare Konsummöglichkeiten schaffen. ${ }^{21}$ Solche liquiditätsbeschränkten Individuen werden bei einer schuldfinanzierten Steuersenkung ihren Konsum erhöhen.

\footnotetext{
${ }^{19} \mathrm{Vgl}$. hierzu Tobin (1981), S. $60 \mathrm{f}$.

${ }^{20}$ Becker (1981) für eine ausführlichere Diskussion der komplexen familiären Beziehungen.

${ }^{21}$ Buiter und Tobin (1980), S. 300. 
Die genaue Auswirkung von Liquiditätsbeschränkungen auf das Neutralitätsergebnis hängt jedoch von deren Ursachen $a b .{ }^{22}$ Verschiedene Autoren begründen das Kapitalmarktversagen nicht explizit, sondern nehmen Liquiditätsbeschränkungen als exogen an: Beispielsweise schlagen sich ungleiche Faktorentlohnung und individuelle Zeitpräferenzen oder Lebenserwartungen in unterschiedlicher Bonität und Zinssätzen nieder. ${ }^{23}$ Eine derartige Heterogenität bedeutet, da $\beta$ unterschiedliche Steuerzahler eine höhere Last der Besteuerung zu verschiedenen Zeitpunkten tragen können. Da alle realen Auswirkungen infolge einer zeitlichen Verschiebung der Steuerlast auf solchen Verteilungseffekten beruhen, sind derartige Erklärungen gewissermaßen tautologisch. Wenn jedoch die Gründe für das Marktversagen explizit erläutert und modelliert werden, ${ }^{24}$ dann läßt sich auch zeigen, daß staatliche Korrekturmaßnahmen durch intertemporale oder interpersonelle Umverteilung die Schuldenneutralität wieder herstellen könnten. Unvollkommene Kapitalmärkte sind deshalb keine hinreichende Bedingung für reale Auswirkungen der Staatsverschuldung, sondern sie haben nur mit Umverteilungseffekten reale Konsequenzen. ${ }^{25}$

\section{d) Umverteilungseffekte der Finanzierungswahl}

Es ist klar, daß eine Umverteilungspolitik Auswirkungen auf die Budgetbeschränkung der Haushalte hat. Selbst wenn Individuen sinen unendlichen Zeithorizont haben und die Kapitalmärkte vollkommen sind, kann das Neutralitätsergebnis verfehlt werden. Dies ist der Fall, wenn eine staatliche Kreditaufnahme eine Umverteilungspolitik ermöglicht (beispielsweise durch Transferzahlungen), welche für Individuen oder Generationen steuerlich verschiedene Konsequenzen hat. ${ }^{26}$ Insbesondere werden reale Effekte einer Staatsschuld verstärkt, wenn Ressourcen an Individuen mit einer höheren marginalen Konsumneigung verteilt werden. ${ }^{27}$ Staatliche Verteilungsmaßnahmen werden jedoch maßgeblich durch das Steuer- bzw. das Ausgabensystem gelenkt.

22 Bernheim (1987), S. 10.

${ }^{23}$ Siehe z. B. Drazen (1978), Hubbard und Judd (1986), Cukierman und Meltzer (1989) und Buiter (1990).

${ }^{24}$ Eine Kreditrationierung, die zu Liquiditätsbeschränkungen führt, läßt sich z. B. aufgrund fehlender Humankapitalmärkte oder asymmetrischer Information erklären, siehe Stiglitz und Weiss (1981) und Yotsuzuka (1987).

${ }^{25}$ Buiter (1990), S. 105ff., sowie Yotsuzuka (1987), S. $431 \mathrm{ff}$.

26 Oft sind jedoch Umverteilungsmaßnahmen und Liquiditätsbeschränkungen voneinander abhängig. Wie bereits erwähnt, könnten Ricardianische Haushalte Umverteilungsversuche neutralisieren, wenn keine Liquiditätsbeschränkungen existieren.

${ }^{27}$ Abel (1986). 


\section{e) Pauschalsteuern}

Das Äquivalenztheorem setzt voraus, daß Änderungen der Staatsschuld nur gegen pauschale Steueränderungen durchgeführt werden. Pauschal- oder Kopfsteuern sind so definiert, daß sie unabhängig vom ökonomischen Handeln des Steuerzahlers entrichtet werden. ${ }^{28}$ Derartige Steuern haben lediglich einen Einkommenseffekt. Da sich kein Relativpreis ändert, gibt es keine Substitutionseffekte. Sie haben folglich die Eigenschaft, daß ihr abdiskontiertes Aufkommen unabhängig vom Zeitpunkt der Steuererhebung ist. Das heißt, sie haben keine intertemporalen Allokationseffekte, was ja auch eine notwendige Bedingung für die Äquivalenz ist.

In der Realität spielen jedoch derartige Pauschalsteuern praktisch keine Rolle. ${ }^{29}$ Die Steuereinnahmen werden vorwiegend durch Einkommen- und Verbrauchsteuern bestritten, deren Aufkommen unelastisch auf Steuersatzänderungen reagiert, weil solche Steuern lastmindernde Aktivitäten auslösen. Eine schuldfinanzierte Einkommensteuersenkung erhöht beispielsweise die Grenzkosten der Freizeit und bietet einen Anreiz, das Arbeitsangebot und somit auch den gegenwärtigen Konsum zu steigern. Bei späteren Steuererhöhungen wird umgekehrt Arbeit für Freizeit und andere unbesteuerte Aktivitäten substituiert. Die gesamten Auswirkungen hängen im wesentlichen von den intertemporalen Substitutionseffekten der ausgewählten Finanzierungswahl ab. ${ }^{30}$

\section{f) Schuldfinanzierung der Zinszahlungen}

Der politische Entscheidungsprozeß wird bei Barros Formulierung des Äquivalenztheorems nicht explizit berücksichtigt, vielmehr sind die fiskalpolitischen Entscheidungsregeln vorgegeben: Bei konstanten Ausgaben besteht einzig die Finanzierungswahl zwischen Steuern einerseits und der intertemporalen Verschiebung der Steuern durch eine Kreditaufnahme andererseits. Die Entscheidung zur staatlichen Kreditaufnahme wird exogen von den Politikern getroffen. Im Unterschied zu finanzwissenschaftlichen Ansätzen, die politische Entscheidungen mit den Wünschen der Haushalte erklären, ${ }^{31}$ spielen diese dabei eine passive Rolle. Sie nehmen die staatliche Spar- bzw. Konsumentscheidung zur Kenntnis und passen sich durch

${ }^{28}$ Siehe z. B. Slemrod (1990).

${ }^{29}$ Eine Ausnahme war die britische Poll Tax. Darauf wird im nächsten Kapitel eingegangen.

30 Barro (1979) selbst untersucht die Auswirkung von verzerrenden Steuern in seinem „Tax Smoothing“ Ansatz. Er zeigt, wie bei verzerrenden Steuern die Staatsverschuldung als Instrument zu Allokationsverbesserungen eingesetzt werden kann. Siehe auch Huber (1990), S. $91 \mathrm{ff}$.

${ }^{31}$ Z. B. das Medianwähler-Modell von Hotelling (1929). Für einen Überblick siehe z. B. Musgrave et al. (1994), Bd. 1, Kap. 6. 
entsprechende Entscheidungen an sie an, um ihren gewünschten Konsumpfad aufrechtzuerhalten.

Deswegen ist eigentlich die politische Finanzierungswahl de facto gar keine. Die Haushalte machen jeden Versuch, die Steuerlast zu verschieben, unwirksam. Unter den im Modell gewählten fiskalpolitischen Regeln führen die Anpassungshandlungen der Haushalte zur politischen Wirkungslosigkeit (sogenannten „Policy ineffectiveness"), weil jeder politische Lenkungsversuch von rationalen, weitsichtigen Individuen durchschaut und neutralisiert wird. ${ }^{32}$ Es stellt sich die Frage, ob eine explizite Berücksichtigung des politischen Entscheidungsprozeßes mit einem breiteren fiskalpolitischen Entscheidungsraum zu anderen, nichtneutralen Ergebnissen führt.

Endogenes politisches Handeln steht z. B. im Mittelpunkt der „Public Choice“ Erklärung der Staatsverschuldung von Buchanan und Wagner (1977). Eine Staatsverschuldung ergibt sich aus dem Zusammentreffen von irrationalen und kurzsichtigen Wählern, welche die späteren Folgen einer Staatsschulderhöhung nicht durchschauen, und opportunistischen, stimmenmaximierenden Politikern, welche die zukünftigen Kosten ihrer Politik nicht berücksichtigen. Einerseits sind Staatsausgaben bei den Wählern beliebt. Deswegen favorisieren Politiker, die auf ihre Wiederwahl hinarbeiten, ihre Erhöhung. Andererseits sind sie bestrebt, eine von den Wählern ungeliebte Steuerfinanzierung zu vermeiden. Die Schuldfinanzierung entpuppt sich als ein Ausweg, der im kurzfristigen politischen Zeithorizont die Einnahmen- und Ausgabenziele miteinander vereinbar macht. Nach dieser Erklärung wird die Finanzierungsoption Staatsverschuldung von den Politikern gewählt, weil diese glauben, daß sie einen Einfluß auf die Höhe der gesamtwirtschaftlichen Nachfrage hat und somit nicht neutral ist.

Ein anderer Erklärungsversuch mit endogenen Staatsausgaben postuliert, daß die Ausgaben durch die Höhe der Steuereinnahmen bestimmt werden. ${ }^{33}$ Demzufolge ersetzen Steuererhöhungen die Defizitfinanzierung nicht, sondern führen bei unveränderter Schuldenfinanzierung zu steigenden Ausgaben. Aus diesem Ansatz wurden die Argumente für Steuersenkungen zum Abbau der Ausgabenquote abgeleitet, welche in den Vereinigten Staaten in den 80 er Jahren u. a. bei Ronald Reagan Unterstützung fanden.

Solche Theorien haben gemeinsam, daß die Wähler aufgrund ihrer Kurzsichtigkeit und Irrationalität die Finanzierungswahl nicht verstehen und in einer „Schuldenil-

\footnotetext{
32 Eine Diskussion über die neuklassischen Modelle, die zum Neutralitätsergebnis und damit auch zur politischen Unwirksamkeit führen, siehe Hoover (1988), S. 138ff.
}

${ }^{33}$ Siehe z. B. Niskanen (1978). 
lusion" glauben, daß eine Staatsschuldenemission sie besser stellte ${ }^{34}$ Erklärungen, welche reale Wirkungen der Staatsverschuldung aufgrund einer derartigen „Schuldenillusion" ableiten, machen sich prinzipiell angreifbar, weil eine solche Verhaltensannahme mit dem grundsätzlichen ökonomischen Postulat der Rationalität nicht vereinbar ist und beliebig viele Ergebnisse ermöglicht. ${ }^{35}$ Sie kann auch wichtige Beobachtungen nicht erklären: Spätestens wenn die Steuererhöhungen dann eintreten, müßte die Schuldenillusion verschwinden. Warum haben denn die historisch unbestrittenen und wiederholten Staatsschulderhöhungen nicht zu Lerneffekten geführt, die eine dauernde Täuschung der Haushalte ausschließen? Warum ist die Höhe der Staatsverschuldung international so unterschiedlich, wenn sie auf eine grundsätzliche (und nicht national unterschiedliche) Irrationalität zurückzuführen ist?

Eine Antwort auf diese Fragen lautet, daß die von den Entscheidungsträgern verfolgte Politik der Staatsverschuldung durchaus den Wünschen von rationalen Wählern entspreche. Wenn das staatliche Entsparen die Wählerpräferenzen widerspiegelt, kann Nichtneutralität, z. B. in polit-ökonomischen Modellen der Staatsverschuldung, auf die Verletzung von anderen Bedingungen der Schuldenneutralität zurückgeführt werden, beispielsweise auf verzerrende Steuern (Alesina und Tabellini [1990]) oder auf unvollkommene Kapitalmärkte (Cukierman und Meltzer [1989]).

Andere Ansätze versuchen, bei Beibehaltung des Rationalitätspostulates die Auswirkungen von politischen Entscheidungsprozessen in die Ausdehnung des fiskalpolitischen Entscheidungsspielraums zu integrieren. ${ }^{36}$ Dies bedeutet, da $\beta$ andere mögliche Finanzierungsquellen in Betracht gezogen werden, d. h. die Endogenisierung der bei Barro (1974) exogenen Komponenten der Budgetbeschränkung, wie z. B. der Geldfinanzierung, zusätzlicher Einnahmen aus Privatisierungserlösen oder der Entdeckung wertvoller natürlicher Ressourcen, sowie Senkungen der Staatsausgaben. Aus historischer Erfahrung wissen rationale Steuerzahler, daß eine staatliche Kreditaufnahme nicht nur künftige Steuererhöhungen bedeuten muß, sondern als Signal einer veränderten Fiskalpolitik gelten kann. Zudem können politische und wirtschaftliche Entwicklungen budgetpolitische Entscheidungen im Zeitverlauf beeinflussen und die Art der Schuldentilgung modifizieren. Wenn alle budgetpolitischen Größen längerfristig im politischen Prozeß endogenisiert werden können, dann wäre es nicht mehr rational, wenn die Haushalte eine Staatsschulder-

${ }^{34}$ Für einen Überblick der Ansätze, welche „Schuldenillusion“ unterstellen, siehe z. B. den Sammelband von Buchanan et al. (1987) und die dort zitierte Literatur.

${ }^{35}$ Diese Kritik machen u. a. Huber (1990), S. 90f., Richter und Wiegard (1993), S. 384 und Alesina und Perotti (1994), S. $10 \mathrm{ff}$.

${ }^{36}$ Siehe z. B. Feldstein (1982), Buiter und Tobin (1980), Leiderman und Blejer (1988), sowie Bohn (1992). 
höhung ausschließlich mit einer zukünftigen Steuererhöhung gleichsetzen. Es stellt sich mithin die Frage, ob bei einer Staatsschulderhöhung die Haushalte zusätzliche Ersparnisse auch bilden, wenn sie annehmen, daß die Verschuldung aus nichtsteuerlichen Quellen getilgt wird.

Die Frage, ob bei einer Geldfinanzierung gegebener Staatsausgaben die Haushalte reale Konsum- und Sparentscheidungen ändern, knüpft an die Diskussion um die Superneutralität des Geldes an. ${ }^{37}$ Wenn Änderungen der Wachstumsrate der Geldmenge keine realen Auswirkungen haben, wird Geld als superneutral beschrieben. Für die Budgetfinanzierung bedeutet die Superneutralität des Geldes eine Ergänzung zum Ergebnis des Äquivalenztheorem, die sich auf die Geldfinanzierung bezieht: Etwaige reale Auswirkungen der Geldfinanzierung, welche eine Veränderung der Inflationsrate auslöst, werden durch Anpassung der Vererbung vollständig kompensiert.

Die klassische Beweisführung der Superneutralität stammt von Sidrauski (1967). Er zeigt in einem Ramsey-Modell, da $ß$ die Konsum- und Sparentscheidungen der Haushalte von der ,modifizierten Goldenen Regel“, d. h. vom Realzins, vom intergenerationalen Diskontfaktor und der Bevölkerungswachstumsrate, abhängen. Die Inflationsrate spielt dagegen keine Rolle. Analog zum Äquivalenztheorem nimmt er an, da $\beta$ die Haushalte einen unendlichen Entscheidungshorizont haben. Drazen (1981) beweist, daß Superneutralität dies voraussetzt. Diese Bedingung ist jedoch nicht hinreichend, ${ }^{38}$ denn trotz Vererbungsmotivs müssen keine positiven Vererbungen auftreten. Michaelis (1993) zeigt, unter welchen Bedingungen das Vererbungsmotiv wirksam und somit Geld superneutral ist. ${ }^{39}$ Die Parallelen zur Staatsschuldneutralität liegen auf der Hand: Wenn die Prämissen des Barro-Modells gelten, ist auch das Geld superneutral und folglich hat auch eine Geldfinanzierung des staatlichen Budgets keine realen Wirkungen. Umgekehrt gilt: Sind die BarroPrämissen verletzt, ist sowohl Staatsverschuldung als auch Geld nicht neutral. ${ }^{40}$

Die dritte Finanzierungsform sind Einnahmen aus den Privatisierungserlösen. Wie im zweiten Kapitel, Abschnitt I.2.c zu zeigen sein wird, sind die budgetpolitischen Wirkungen dieser Einnahmen mit der Staatsverschuldung gleich. Wird dies von den Haushalten erkannt, gilt die Analogie zur Schuldenneutralität ebenfalls für diese Finanzierungsform.

\footnotetext{
${ }^{37}$ Zur Superneutralităt des Geldes siehe z. B. Orphanides und Solow (1990), S. $236 \mathrm{ff}$.

${ }^{38}$ Siehe hierzu Haliassos und Tobin (1990), S. 932ff.

${ }^{39}$ Die Auswirkungen von Umverteilungsmaßnahmen oder Pauschalsteuern sind offensichtlich invariant von der Finanzierungsform.

${ }^{40}$ Eine ausführlichere Diskussion, die auf die Interaktion von Staatsschuld und Geldschöpfung eingeht, findet sich bei Huber (1990). S. 105ff. 
Besondere Relevanz hat die vierte staatliche Einnahmequelle, die natürlichen Ressourcen, z. B. im Zusammenhang mit der Entdeckung der britischen Nordseeölvorräte. Dies wirkt sich positiv auf die Produktivität aus und erhöht dadurch das Wirtschaftswachstum. Somit werden die Vererbenden erwarten, daß im Wachstumsprozeß das Nutzenniveau ihrer Nachfolger deswegen zunehmen wird. Wie bereits unter a) diskutiert, werden bestimmte Individuen diese Wohlstandsverbesserung bei ihrem Vererbungsverhalten berücksichtigen und sich bei der Randlösung befinden, wo sie keine Erbschaften hinterlassen. Die Staatsverschuldung wird nicht mehr neutralisiert.

Schließlich ist zu untersuchen, ob eine Senkung der Staatsausgaben durch kompensierende Maßnahmen der Haushalte neutralisiert wird. Bei Barro (1974) bestehen die Ausgaben aus pauschalen Transferzahlungen. Die Staatsausgaben haben damit den Charakter von negativen Pauschalsteuern. Analog zu den unter e) ausgeführten Bedingungen der nichtverzerrenden Steuern sind Ausgaben neutral, wenn sie keine Substitutionswirkungen hervorrufen. Ist dies der Fall, dann verliert das Äquivalenztheorem bei einer Senkung der Staatsausgaben seine Gültigkeit nicht.

Die Ausgaben lassen sich jedoch auch als vom Staat bereitgestellte Güter interpretieren. Bei gegebenen Ausgaben wird die Äquivalenzaussage in bezug auf die Finanzierung dieser Ausgaben aufrechterhalten. Eine Senkung der realen Ausgaben zur Finanzierung der Zinszahlungen beruht die intertemporale Aufteilung der Steuer- und Schuldenlast nicht, was für die Finanzierungsneutralität spricht. Die Analyse muß jedoch um eine zusätzliche Dimension erweitert werden, weil neben den Substitutionsbeziehungen zwischen den intertemporalen Finanzierungsquellen auch die Substitutierbarkeit zwischen privaten und staatlichen Ausgabentätigkeiten berücksichtigt werden muß. Die Ausgabensenkung löst einen Netto- Einkommenseffekt, bzw. Vermögenseffekt bei den Haushalten aus. Dabei ist es möglich, daß ihre Konsum- oder Sparentscheidungen die Äquivalenzaussage verletzen. Dieser $\mathrm{Zu}-$ sammenhang wird in bezug auf Erhöhungen der Staatsausgaben üblicherweise als direktes „Crowding Out“41 erörtert.

Direktes "Crowding Out" tritt auf, wenn staatliche Ausgaben eine unmittelbare Wirkung auf private Entscheidungen hervorrufen und dadurch privatwirtschaftliche Aktivitäten verdrängen; bzw. wenn die Staatshandlung durch kompensierende Maßnahmen des Privatsektors vollständig neutralisiert wird. Denkbar in diesem Zusammenhang sind Entscheidungen, die das verfügbare Einkommen betreffen, z.B. die Substitution steuerfinanzierter Altersversorgung durch private Rentenversicherungen, oder Konsumausgaben für staatliche statt privater Bildungs- und Ge-

${ }^{41}$ Das Phänomen wurde von David und Scadding (1974) untersucht. Siehe auch Buiter (1977) und

Buiter und Tobin (1980) sowie die dort erwähnte Literattr Safford - 978-3-631-75199-2 
sundheitsgüter, bzw. staatliche statt privater Investitionsausgaben für Humankapital.

Wie die Staatsschuldneutralität tritt „Staatsausgabenneutralität" nur unter bestimmten restriktiven Bedingungen auf. Erstens müssen private und staatliche Ausgaben vollkommene Substitute sein. Zweitens haben private und staatliche Ausgaben identische Einkommenswirkungen, und die Haushalte unterliegen diesbezüglich keiner „Ausgabenillusion“. ${ }^{42}$ In einer vollbeschäftigten geschlossenen Wirtschaft ersetzt die staatliche Inanspruchnahme von Ressourcen immer die private Verwendung. Aber damit ist nicht geklärt, welche Ausgaben und mit welchen Konsequenzen verdrängt werden. Sämtliche reinen öffentlichen Güter mit den Eigenschaften der Nicht-Rivalität und Nicht-Ausschließbarkeit im Konsum sind ex definition nicht durch private Güter substituierbar. Somit ist anzunehmen, daß sie kein direktes „Crowding-Out" verursachen.

Die Auswirkungen von Ausgabenkürzungen für reine öffentliche Güter auf die Ricardianische Äquivalenz wurden bislang nicht näher erforscht. Jedoch, wenn sie kein direktes „Crowding-Out" verursachen, läßt sich die Hypothese formulieren, $\mathrm{da} B$ das Neutralitätsergebnis verfehlt wird. Handelt es sich dagegen bei den Staatsausgaben um Güter, die durch ein privates Angebot substituiert werden, wie dies gewöhnlich der Fall ist, so gelten sowohl Staatsausgaben- als auch Staatsschuldneutralität. ${ }^{43}$

\section{3) Empirische Untersuchungen}

Trotz unzähliger Untersuchungen liefert die empirische Evidenz noch keine eindeutige Antwort auf die Gültigkeit des Äquivalenztheorems. Ohne hier im Detail auf die empirischen Ergebnisse einzugehen, ${ }^{44}$ kann folgendes festgehalten werden.

Während die große Bedeutung von intergenerationellen Transfers das Äquivalenztheorem unterstützt, ${ }^{45}$ überzeugt die Evidenz eines altruistischen Vererbungsmotivs weit weniger. ${ }^{46}$ Ein signifikanter Anteil der intergenerationellen Transfers

42 D. h. analog zur Schuldenillusion müssen sich die Haushalte „ultrarational“ verhalten, David und Scadding (1974).

${ }^{43}$ Buiter (1977), S. 311 f.

44 Überblicke der empirischen Forschung finden sich bei Bernheim (1987), de Haan und Zelhorst (1988), sowie Seater (1993).

${ }^{45}$ Nach Kotlikoff und Summers (1981) besteht die Kapitalbildung in den U.S.A. zu 80\% aus intergenerationalen Transferzahlungen.

${ }^{46}$ Bernheim (1987), S. 20ff und Wilhelm (1996). 
scheint entweder ungeplant oder strategischer Natur zu sein. Poterba und Summers (1987) greifen die Prämisse der altruistischen Vererbung aus einer ganz anderen Perspektive an. Sie stellen die Relevanz eines intergenerationellen Planungshorizonts in Frage. Ihre Simulationen, sowie z. T. die historische Erfahrung in den USA zeigen, daß Zunahmen der staatlichen Schuldenbestände in relativ kurzer Zeit, d. h. innerhalb einer Generation, wieder abgebaut worden sind. ${ }^{47}$ Somit ist ihrer Ansicht nach die Annahme des ewigen Generationenvertrages von geringerer Bedeutung für die Äquivalenzaussage. ${ }^{48}$

Eine Reihe empirischer Studien untersuchen die Bedeutung von Liquiditätsbeschränkungen. ${ }^{49}$ Die meisten finden starke Konsumeffekte der Defizitfinanzierung, welche aus einer Entlastung der Budgetbeschränkung für liquiditätsbeschränkte Individuen resultieren könnten. Derartige Effekte könnten sich auch aus der Irrationalität zumindest einiger Individuen erklären, welche die zukünftige Zinszahlungslast übersehen. In beiden Fällen, Liquiditätseffekten und Irrationalität, wird jedoch das Neutralitätsergebnis verfehlt.

Schließlich haben Zeitreihenanalysen und internationale Vergleichstudien versucht, in der Entwicklung von gesamtwirtschaftlichen realen und nominalen Variablen direkte Beweise für die Nichtneutralität zu finden. ${ }^{50}$ Trotz methodischer und statistischer Schwierigkeiten wird von zahlreichen Kommentatoren der gemeinsame Anstieg von Budgetdefiziten, Zinssätzen und Konsum in den 80er Jahren als Wechselbeziehung und damit als indirekter Beweis für die Nichtneutralität der Verschuldung interpretiert. ${ }^{51}$

\section{III. „Crowding out" Effekte der Staatsverschuldung}

Die Diskussion im letzten Abschnitt zeigt, daß Staatsverschuldung nur unter sehr restriktiven Bedingungen neutral sein kann. Aus dem Umkehrschluß ist dann anzu-

${ }^{47}$ Interessanterweise hat sich Ricardo (1851) S. 247 die Steuervermeidung nicht durch den Tod vorgestellt, wie praktisch alle die nachfolgenden Analysen annehmen, sondern durch Emigration. Schließlich hat auch er den relevanten Zeithorizont für die Äquivalenz als innerhalb eines Lebenszyklus betrachtet.

48 Die großen Schuldentilgungen in Europa bis 1945 fanden ebenfalls innerhalb eines kurzen Zeitraums statt, Alesina (1988).

49 Insbesondere sind die Studien über die U.S.A. von Hubbard und Judd (1986) und Zeldes (1989) hervorzuheben. Für die BRD siehe Kitterer (1986).

${ }^{50}$ Siehe Bernheim (1987), S. 34ff.

${ }^{51}$ Siehe insbesondere IMF (1995a) S. 83ff. 
nehmen, da $\beta$ die Staatsverschuldung ökonomische Auswirkungen hat und da $\beta$ die öffentliche Diskussion darüber gerechtfertigt ist. Im diesem Abschnitt wird die Keynesianische Theorie über den Einsatz von Defiziten zur Erhöhung der Nachfrage in einer unterbeschäftigten Volkswirtschaft dargestellt.

Eine schuldfinanzierte Steuersenkung und/oder ein schuldfinanzierter Ausgabenanstieg erhöht nach den Keynesianischen Wirkungszusammenhängen das verfügbare Einkommen und regt dadurch die Gesamtnachfrage an. Voraussetzung dafür sind Vermögens- bzw. Einkommenseffekte, welche die Haushalte zu zusätzlichen Ausgaben bewegen. „Crowding Out“ findet statt, wenn ein expansiver fiskalpolitischer Impuls einen Rückgang privatwirtschaftlicher Aktivitäten verursacht und dadurch die Wirkung der Fiskalpolitik mindert, neutralisiert oder sogar ins Negative umkehrt. $^{52}$

\section{1) Kurzfristiges Crowding Out}

Steuer- oder Ausgabemaßnahmen wirken indirekt über Preis- oder Zinsänderungen oder aufgrund der Aufhebung von Budgetbeschränkungen auf das Einkommen. Diese Auswirkungen werden als ,indirektes Crowding Out“ beschrieben.

\section{a) Crowding Out im IS-LM Modell}

Im Keynesianischen IS-LM Unterbeschäftigungsmodell werden die kurzfristigen Impulse der Fiskalpolitik analysiert. Der Output wird durch die aggregierte Nachfrage bei gegebenem Preisniveau determiniert, da annahmegemäß bei der kurzfristigen Betrachtung freie Kapazitäten bestehen, welche die Nachfrageentfaltung ohne Preisänderungen akkomodieren können. Obwohl Erwartungen an und für sich eine wichtige Rolle in der Keynesianischen Theorie zukommen, werden sie in diesem Modell nicht explizit berücksichtigt: ${ }^{53}$ Erwartete, zukünftige wirtschaftliche Entwicklungen haben keinen Einflu $ß$ auf die Einkommensbestimmung der Gegenwart. Darüber hinaus werden die aus der Veränderung von Stromgrößen resultierenden Bestandsänderungen als vernachlässigbar angesehen. So werden trotz Nettoinvestitionen ein konstanter Kapitalstock und trotz staatlicher Budgetdefizite unveränderte Vermögensbestände zwischen dem Staat und den Haushalten unterstellt.

\footnotetext{
52 Buiter (1985), S. $41 \mathrm{ff}$.

${ }^{53}$ In seiner „General Theory“ räumt Keynes (1936) Erwartungen eine ganz zentrale Rolle ein; z. B. im Kapitel 5 „Expectation as determining output and employment“ sowie in Bezug auf die Investitionstätigkeit im Kapitel 12 „Long-term expectation““th Safford - 978-3-631-75199-2
} 
Die Einkommenswirkung und der „Crowding Out“ Effekt der Keynesisanischen Fiskalpolitik lassen sich graphisch darstellen:

\section{Abbildung 2: Der kurzfristige Effekt einer expansiven Fiskalpolitik}

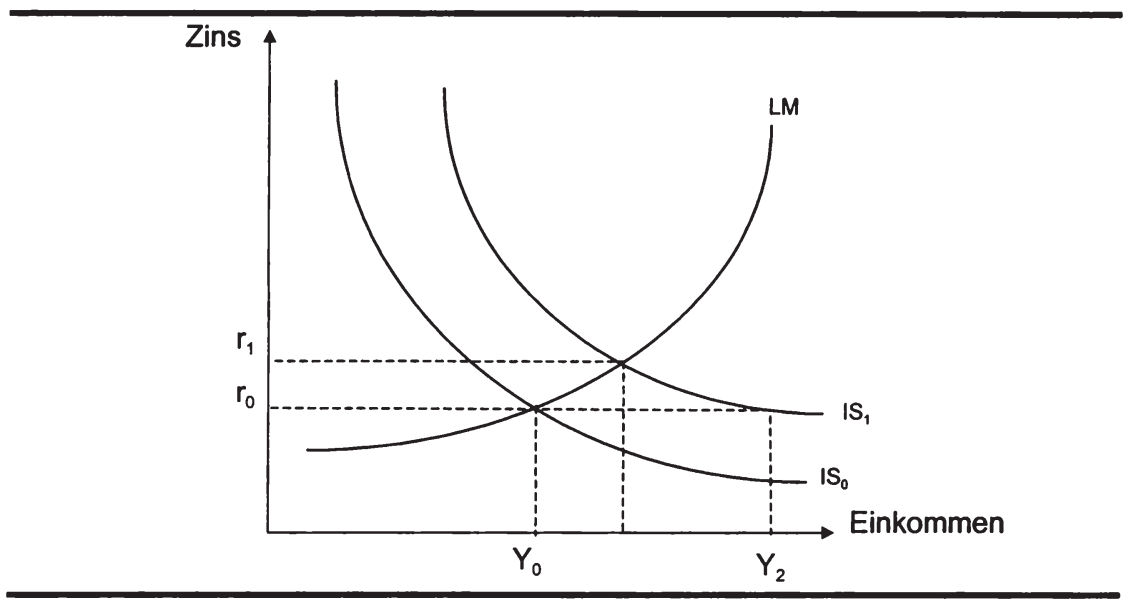

Während die IS-Kurve (Investitionen = Sparen) das Gütermarktgleichgewicht wiedergibt, beschreibt die LM-Kurve (Liquiditätspräfenz = Geldangebot) alle Kombinationen von Einkommen und Zins, bei denen sich der Geldmarkt im Gleichgewicht befindet. Eine schuldfinanzierte Steuersenkung erhöht das verfügbare Einkommen und damit den Konsum. Die daraus resultierenden expansiven Einkommenswirkungen führen zu einer Rechtsverschiebung der IS-Ortslinie von ihrem Ausgangspunkt $Y_{0}$. Bei einem gleichbleibenden Zinssatz steigt das Einkommensniveau auf $Y_{2}$. Der Konsumanstieg hat jedoch auch Konsequenzen auf den Geldmarkt, da er zu einer Erhöhung der Nachfrage nach Transaktionskasse führt. Bei konstantem Geldangebot steigt der Zins, wodurch zinselastische Investitionen verdrängt werden. Das neue Gleichgewichtseinkommen befindet sich bei $Y_{1}$ : Die Fiskalpolitik wirkt trotz „Crowding-Out" noch positiv auf das Einkommen. Die Elastizitäten der unterschiedlichen Parameter und somit das Ausmaß des „CrowdingOut" spiegeln sich in der jeweiligen Steigung der IS-LM Kurven wider.

\section{b) Klassisches „Crowding Out“}

Die oben dargestellte Fiskalpolitik stützt sich auf die Keynesianische Annahme unterbeschäftigter Kapazitäten. „Crowding Out“ findet lediglich in dem Maße statt, in dem die geld- und fiskalpolitischen Steuerungsinstrumente nicht aufeinander abgestimmt werden. Dagegen sorgt bei der „klassischen“ Betrachtung der Preisme- 
chanismus für stets geräumte Märkte und somit für die Vollauslastung des Produktionspotentials. Die Geldnachfrage wird nach quantitätstheoretischer Vorstellung allein vom Einkommensniveau und nicht vom Zins bestimmt. Mit einer vollkommen zinsunelastischen Geldnachfrage ist der Geldmarkt nur bei Vollbeschäftigungseinkommen im Gleichgewicht. Im IS-LM Schema wird dieser Zusammenhang durch eine senkrechte LM-Kurve dargestellt. Eine expansive Fiskalpolitik kann keine Auswirkung auf das Output haben. Längerfristig könnten Steuersenkungen im Sinne von „supply side“ Maßnahmen den Output erhöhen. Expansive Fiskalpolitik wird jedoch auch hier nicht benötigt, um freie Kapazitäten auszunutzen, da diese in der Klassischen Welt sofort Verwendung finden, bzw. nicht vorhanden sind.

\section{2) „Crowding Out“ unter Berücksichtigung rationaler Erwartungen}

Wie bereits erwähnt, findet die Rolle von Erwartungen im herkömmlichen IS-LM Modell keine Berücksichtigung. In der Realität dagegen sind fiskalpolitische Maßnahme schon in der Regel vor ihrer tatsächlichen Implementierung allgemein bekannt. ${ }^{54}$ Das hier vorgestellte Modell zur Untersuchung der Effizienz der Fiskalpolitik bedient sich der Hypothese der rationalen Erwartungsbildung und untersucht die Portfolioeffekte und das Investitionsverhalten bei einer antizipierten fiskalpolitischen Expansion. ${ }^{55}$

In diesem Zusammenhang postuliert die Hypothese der rationalen Erwartungsbildung, daß sobald die Information über eine geplante, glaubwürdige, fiskalpolitische Expansion für alle verfügbar ist, diese optimal verwendet wird, um die zukünftige Wirtschaftsentwicklung zu prognostizieren. Die erwarteten Auswirkungen werden dann unmittelbar - folglich auch vor der tatsächlichen Durchführung der geplanten Maßnahme - bei privaten Entscheidungen berücksichtigt. Die Erwartungsbildung läßt sich im IS-LM Modell erfassen durch die Unterscheidung zwischen kurz- und langfristigen Zinssätzen sowie die Annahme unterschiedlicher Anpassungsgeschwindigkeiten der endogenen Variablen nach einer Systemstörung: In diesem Falle paßt sich das IS Gleichgewicht nach einer Erhöhung der Staatsausgaben langsam an. Das Geldmarktgleichgewicht wird durch den LM Zusammenhang beschrieben: Bei gegebenem Output bestimmt die reale Kassenhaltung den

\footnotetext{
${ }^{54}$ Blanchard (1981) S. 137.

${ }^{55}$ Die formale Darstellung findet sich bei Blanchard (1981). Buiter (1985) S. 49ff. berücksichtigt bei einer ähnlichen Analyse die staatliche Budgetrestriktion, um die Auswirkungen einer Schuldfinanzierung explizit einzubeziehen. Begg (1982) S. 172ff. untersucht Vermögenseffekte und die Auswirkungen auf Konsumverhalten bei Stabilisierungspolitik mit rationalen Erwartungen.
} 
kurzfristigen Zins. Die Investitionen hängen positiv von Tobins „q“ ab, d. h. von der Beziehung zwischen der Grenzleistungsfähigkeit des Kapitals und seiner Marktertragsrate [Tobin (1969)] - und somit vom langfristigen Zins. Eine intertemporale Arbitragebedingung verbindet diesen langfristigen Zins mit dem Durchschnitt der erwarteten, kurzfristigen Zinssätze. ${ }^{56}$ Bei sicherer und vollkommener Voraussicht gleichen sich die erwarteten und tatsächlichen Renditen. ${ }^{57}$ Dadurch ist es möglich, daß erwartete, zukünftige Entwicklungen das gegenwärtige Outputniveau beeinflußen können.

Nach Bekanntgabe der künftigen, expansiven Fiskalpolitik werden entsprechend dem Keynesianischen Modell ein kurzfristiger Zinsanstieg und eine Outputerhöhung erwartet. Ersterer erhöht die erwarteten, kurzfristigen Zinssätze und damit durch die Zinsarbitragebedingung auch den gegenwärtigen, langfristigen Zins. (Die IS-Ortslinie bewegt sich nach links.) Dagegen gehen aufgrund der erwarteten Outputerhöhung positive Impulse auf die Nachfrage aus ${ }^{58}$ (eine Rechtsverschiebung der IS Kurve). Da die Fiskalpolitik noch unverändert ist, hängt die gesamte Auswirkung von der jeweiligen Stärke dieser gegenläufigen Effekte ab. Überwiegt der Zinseffekt, dann gehen die zinsabhängigen Ausgaben zurück, ${ }^{59}$ danach sinkt auch die Gesamtnachfrage. Der resultierende Outputeinbruch überträgt sich über die Transaktionskasse auf die kurzfristigen zukünftigen Zinssätze, die wieder sinken. Erst nach tatsächlicher Einführung der Fiskalpolitik steigt der Output nach herkömmlichem Muster wieder. Die kontraktive Wirkung tritt verstärkt auf, wenn Vermögensbestandsänderungen beim Anpassungsprozeß zusätzlich berücksichtigt werden. Die Erwartung steigender kurzfristiger Zinssätze infolge des staatlichen Finanzierungsbedarfs erhöht den langfristigen Zinssatz dermaßen, daß während des Anpassungsprozesses private Investitionsausgaben unter ihr Ausgangsniveau verdrängt werden können. ${ }^{60}$

Die hier angestellten Überlegungen zeigen, daß die Nachfragewirkungen der Fiskalpolitik u. U. schwieriger zu prognostizieren sind, als das traditionelle IS-LM Modell dies nahelegt. ${ }^{61}$ Schwankende und instabile Erwartungen können sich auf

${ }^{56}$ Die Verbindung zwischen kurz- und langfristigen Zinssätzen beruht auf der Erwartungstheorie der Zinsstruktur. Eine ausführliche Darstellung befindet sich bei Kath (1972).

57 Von unterschiedlichen Risiken zwischen den verschiedenen Vermögensformen wird hier abstrahiert.

${ }^{58}$ Z. B. durch eine positive Kursentwicklung, die einen Vermögenseffekt auf den Konsum hervorbringt und entsprechend Tobin's q auch die Investitionen anregt, vgl. Blanchard (1981), S. 132.

${ }^{59}$ Bzw. die Börsenentwicklung ist negativ.

${ }^{60}$ Buiter (1985) S. $73 \mathrm{ff}$.

${ }^{61}$ Dieses Modellergebnis findet nach Ansicht einiger Autoren historische Unterstützung durch die amerikanische Steuerreform 1981 und die anschließende Rezession. Siehe hierzu Blanchard und Dornbusch (1986a), S. 163ff. 
die Einkommensentwicklung übertragen. Daraus lassen sich Argumente für einen Verzicht auf kurzfristige Stabilisierungspolitik zugunsten einer vorher angekündigten und glaubwürdigen, regelgebundenen Politik ableiten. ${ }^{62}$ Die Unsicherheit über die kurzfristige Multiplikatorwirkung hat auch Konsequenzen für die Fiskalpolitik in einer längerfristigen Sicht. Eine Strategie mußte nicht nur die Wirkung der Fiskalpolitik in der gegebenen Periode erfassen, sondern auch alle zukünftigen, erwarteten Politikmaßnahmen berücksichtigen. ${ }^{63}$ Glaubwürdig und stabilitätsfördernd wirkt eine Fiskalpolitik nur dann, wenn die Stabilität der staatlichen Budgetrestriktion nicht nur für die gegebene Periode gewährleistet ist, sondern auch für alle zukünftigen Perioden, über die Erwartungen gebildet werden.

\section{3) Langfristiges „Crowding-Out“}

In einer längerfristigen Perspektive hat die Fiskalpolitik nicht nur diese von Friedman (1987) "first-round effects“ genannten Auswirkungen auf Stromgrößen, sondern ruft Vermögenseffekte hervor, die mit den Bestandsänderungen infolge der Finanzierung zusammenhängen. Die monetaristische Kritik der aktiven Fiskalpolitik betont eine längerfristige Sicht, bei der kontraktive, dauerhafte Finanzierungswirkungen, die expansiven, kurzfristigen Impulse überwiegen. Einige Beiträge, ${ }^{64}$ unter denen insbesondere die Arbeit von Blinder und Solow $(1973,1974,1976) \mathrm{zu}$ nennen sind, haben versucht, die Wirksamkeit der Fiskalpolitik unter Berücksichtigung von Vermögenseffekten zu bestätigen.

Blinder und Solow $(1973,1976)$ untersuchen Vermögensänderungen infolge der Finanzierung zusätzlicher Staatsausgaben, sowie ihre Aufteilung zwischen Geld, Bonds und Kapitalstock. ${ }^{65}$ In der folgenden Analyse wird das Blinder/Solow (1973) Modell übernommen mit einer Modifikation bei der Spezifizierung der Investititionen. Neben Zins und Kapitalstock hängen sie auch vom Einkommensniveau ab. ${ }^{66}$ Ein einfaches Akzelerationsprinzip, welches die Abweichung zwischen tatsächlichem und optimalem Kapitalstock schließt, beeinflußt somit die Höhe der Investitionen. Es werden die Auswirkungen einer einmaligen Erhöhung der Staats-

62 Siehe z. B. Schelling (1982).

${ }^{63}$ Van Velthoven, Verbon und van Winden (1993), S. 5.

${ }^{64}$ Die ersten Beiträge, die Vermögenseffekte untersuchten, entstanden bereits in den 60er Jahren, siehe z. B. Ott und Ott (1965) und Christ $(1967,1968)$.

${ }^{65}$ Weiterhin besteht die Annahme über freie Kapazitäten, weshalb das Preisniveau konstant bleibt. Weiter wird auch die Erwartungsbildung als exogen betrachtet.

${ }^{66} \mathrm{Vgl}$. hierzu die Kritik der Investitionsspezifizierung in Blinder/Solow (1973) in Tobin und Buiter (1990), S. 258. 
ausgaben bei konstantem Steuersatz finanziert ausschließlich durch die Ausgabe von Bonds untersucht.

Die Analyse erfolgt im erweiterten IS-LM Modell. Bei der Gütergleichgewichtskurve (IS-Kurve) hängt der Konsum vom verfügbaren Einkommen, einschließlich der Zinszahlungen auf die Staatsschuld sowie vom realen Vermögen ab. Die Nachfrage nach Realkasse (LM-Kurve) wird ebenfalls um das Vermögen als Einflußfaktor erweitert.

Der von Blinder/Solow (1973) unterstellte Anpassungsvorgang an die expansive Politik läßt sich verbal wie folgt beschreiben.$^{67}$ Die einmalige Erhöhung der Staatsausgaben verursacht einen Budgetdefizit. Die Bondsausgabe hat einen positiven Einkommenseffekt. Demzufolge steigen die einkommensabhängigen Steuereinnahmen und tragen zum Abbau des Budgetdefizits bei. Der verbleibende Schuldenbestand macht die Emission zusätzlicher Bonds für den Schuldendienst in der folgenden Periode notwendig. Die Bondsausgabe löst Vermögenseffekte aus, die weitere expansive Effekte mit sich bringen. Wenn die Steuereinnahmen schneller als der Schuldendienst wachsen, sinkt das durch Bonds zu finanzierende Defizit stetig ab, bis es komplett abgebaut wurde.

Dies ist jedoch nur eins von drei möglichen Ergebnissen. ${ }^{68}$ Die Anpassung an das neue Gleichgewicht hängt von zwei Bedingungen ab. Erstens ist es notwendig, da $\beta$ die Bondsausgabe expansiv auf das Einkommen wirkt $(\partial \mathrm{Y} / \partial \mathrm{B}>0)$. Dieser Einkommenseffekt muß zweitens ausreichend groß sein, um laufend höhere Steuereinnahmen einzubringen, damit das Budgetdefizit im Zeitverlauf abnimmt. Das heißt, bei zunehmender Bondsausgabe muß sich der Finanzierungsbedarf des Budgetdefizits stetig verringern $(\mathrm{d} \dot{\mathrm{B}} / \mathrm{dB}<0)$.

Die Auswirkung der Bondsausgabe auf das Einkommen hängt vom Einkommenseffekt auf den Konsum und von den Vermögenseffekten auf Konsum und Geldnachfrage ab. Wenn als Extremfall der Vermögenseffekt auf die Geldnachfrage die Einkommens- und Vermögenseffekte auf den Konsum überwiegt, kann die Wirkung der Bondsausgabe auf das Einkommen sogar negativ sein. In diesem Fall wird die Verschuldung explodieren. Abbildung 3 verdeutlicht den Zusammenhang graphisch. Bis zum Zeitpunkt $t_{1}$ ist das Budget ausgeglichen. Danach wird eine schuldfinanzierte Ausgabenerhöhung (G) getätigt. Wirkt die Defizitpolitik kontraktiv, sinken die einkommensabhängigen Steuereinnahmen (T). Das Budgetdefizit, das zur Finanzierung der zusätzlichen Ausgaben entstand, wird nicht durch die

${ }^{67}$ Auf die formale Ableitung wird an dieser Stelle verzichtet. Sie findet sich im Anhang I.

${ }^{68}$ Vgl. Blinder und Solow (1973), S. 328. 
Steuereinnahmen abgebaut, sondern steigt aufgrund der sinkenden Steuereinnahmen und der um die Zinslast exponential anwachsenden Budgetdefizite.

\section{Abbildung 3: Kontraktive Wirkung der Defizitpolitik}

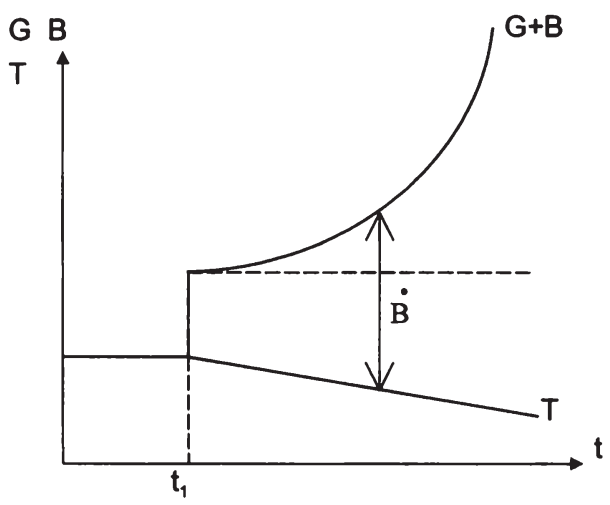

Abbildung 4 veranschaulicht den zweiten Fall. Die Bondsausgabe hat einen positiven Einkommenseffekt, jedoch ist dieser nicht ausreichend groß, um die Budgetlücke zu schließen $(\mathrm{d} \dot{\mathrm{B}} / \mathrm{dB}>0)$. Das System ist wiederum instabil.

\section{Abbildung 4: Instabilität bei expansiver Wirkung der Defizitpolitik}

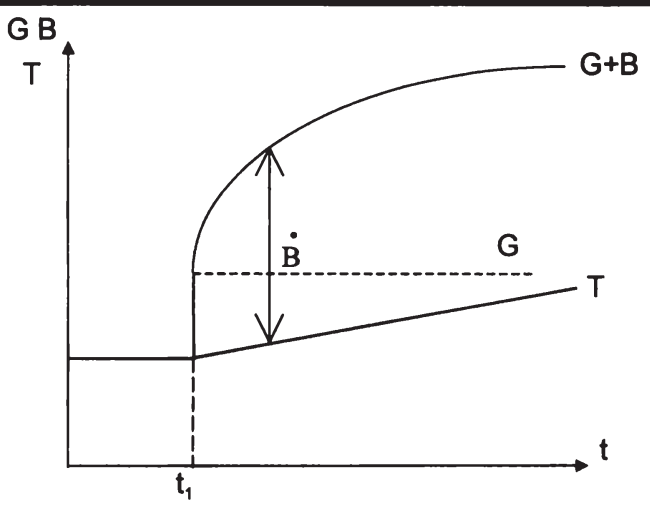

Das stabile Szenario ist in der Abbildung 5 aufgezeigt. Die expansive Einkommenswirkung der Bondsausgabe reicht aus, um das entstandene Budgetdefizit wie- 
der abzubauen (beide Bedingungen sind erfüllt). In $t_{2}$ ist ein neues Gleichgewicht bei einem höheren Einkommen erreicht.

\section{Abbildung 5: Stabilität bei expansiver Wirkung der Defizitpolitik}

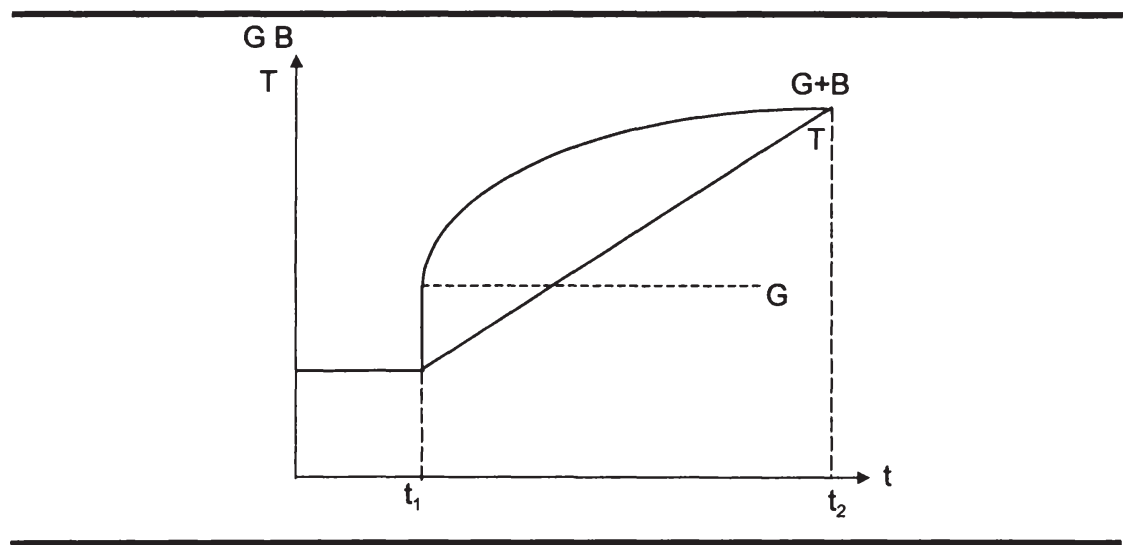

Im ursprünglichen Blinder/Solow Modell (1973) sind die Ergebnisse relativ robust und lassen das dritte Szenarium erwarten. Die Stabilitätsbedingungen lassen sich in Elastizitäten umformen und liefern dann ökonomisch nachprüfbare Größen, mit denen Blinder und Solow beweisen, daß unter realistischen Annahmen Stabilität gegeben ist. ${ }^{69}$

Durch die Berücksichtigung der Einkommensabhängigkeit der Investitionen werden die Stabilitätsbedingungen jedoch erheblich strenger (siehe hierzu den Anhang) und sind sehr empfindlich gegenüber Parameterwerten des Modells. Im langfristigen Unterbeschäftigungsmodell wirkt eine bondfinanzierte Staatsausgabenpolitik nur unter sehr restriktiven Bedingungen einkommenserhöhend. Tendiert das System nicht zu einem stabilen Gleichgewicht, dann nehmen die Defizite unbegrenzt zu: Die Verschuldung explodiert. Sind alle Stabilitätsbedingungen erfüllt, dann wirkt die bondfinanzierierte Fiskalpolitik $u$. U. restriktiv. Zahlreiche Erweiterungen und Varianten des Blinder/Solow (1973) Modells führen zum selben theoretischen Ergebnis: ${ }^{70}$ Stabilität ist unmöglich (oder unwahrscheinlich), wenn eine Staatsausgabenpolitik durch Bondsemission finanziert wird. Dieser Befund muß die stabilitätspolitische Begründung für defizitfinanzierte Staatsausgaben in Frage stellen.

\footnotetext{
${ }^{69}$ Blinder und Solow (1974) S. 54

${ }^{70}$ Ein Überblick der Literatur und eine ausführliche Diskussion der unterschiedlichen Modelle befinden sich bei Christ (1979). 
Ursache für dieses Ergebnis ist erstens die zwangsläufige Schuldfinanzierung der Defizite, welche diese in den folgenden Perioden unvermeidbar um die Zinszahlungen erhöhen. Eine höhere Verschuldung führt zu einem höheren Schuldendienst. Eine kontinuierliche fiskalische Expansion resultiert, die sich zu einem explosiven Schuldenanstieg entwickeln kann. Zweitens ist dieses Szenario möglich, weil die fiskalpolitische Regel im Modell konstante Staatsausgaben und Steuersätze spezifiziert. Können beispielsweise die Steuern erhöht oder die ansteigenden Schuldzinsen durch eine kompensierende Senkung der Staatsausgaben wettgemacht werden, dann läßt sich die Modellstabilität wieder erreichen. ${ }^{71}$ Angesichts dieser Politikwahl stellt Buiter fest:

„an explosive debt-deficit spiral is a policy choice rather than a deep structural property of the economy. “72

\section{Auswirkungen der Staatsverschuldung auf die Kapitalbildung}

Die oben dargestellte Keynesianische Analyse ging von einer unterbeschäftigten Wirtschaft aus. Eine staatliche Schuldenaufnahme stellt vor diesem Hintergrund eine vorübergehende fiskalpolitische Maßnahme dar, welche durch die Belebung der gesamtwirtschaftlichen Nachfrage das Vollbeschäftigungsniveau wieder zu erreichen versucht. Es ging also um die Auswirkungen von temporären Defiziten.

Bei der folgenden Betrachtung dagegen wird von Vollbeschäftigung ausgegangen. Die Wirtschaft befindet sich im sogenannten „steady state“, d. h. auf einem gleichgewichtigen Wachstumspfad. Die folgenden Ansätze betrachten somit eine längerfristige Perspektive und untersuchen die Auswirkungen von permanenten staatlichen Defiziten auf die Kapitalbildung in einer Volkswirtschaft. Dabei wird versucht, die verschiedenen Auswirkungen auf intuitive und anschauliche Weise verbal darzustellen. Auf eine formale Ableitung der verschiedenen Effekte wird verzichtet. $^{73}$

\footnotetext{
${ }^{71}$ Vgl. Christ (1979) S. 534f.

72 Buiter (1985) S. 45; Kursivschift im Originaltext (J.S.).
}

${ }^{73}$ Hierfür sei auf die zahlreiche Literatur verwiesen. Insbesondere zu erwähnen sind Atkinson und Stiglitz (1980), Carlberg (1988) und Kitterer (1988)udith Safford - 978-3-631-75199-2 


\section{1) Der Transferansatz: Die Last der Auslandsverschuldung}

Die Auswirkungen der Staatsverschuldung wurden in den Jahren nach dem 2. Weltkrieg im Rahmen der sogenannten „Lastdebatte“ erörtert. ${ }^{74}$ Es wurde versuchte, die vorherrschende klassische Ansicht zu widerlegen, wonach öffentliche und privatwirtschaftliche Verschuldung die gleichen Auswirkungen haben. ${ }^{75}$ In einer geschlossenen, vollbeschäftigten Wirtschaft lassen sich die gesamtwirtschaftlichen Konsummöglichkeiten durch die Verschuldung nicht erhöhen, weil eine geschlossene Wirtschaft nicht über mehr Güter und Leistungen verfügen kann, als sie selbst produziert: Ressourcen für zusätzliche staatliche Ausgaben können nur von gegenwärtigen privaten Verwendungen entzogen werden. Dagegen erlaubt eine Auslandsverschuldung durch den Bezug ausländischer Ressourcen eine Konsumzunahme, ohne den privaten, inländischen Konsum zu belasten.

Aufgrund dieser Überlegungen wurde die Last der Staatsverschuldung als staatliche Inanspruchnahme der privaten Ressourcen abgeleitet. Bei einer Inlandsverschuldung kann ein derartiger Ressourcenentzug weder in die Zukunft verlagert, noch vom Finanzierungsmodus bestimmt werden. Auch die Schuldzinszahlungen bedeuten keinen gesellschaftlichen Wohlfahrtsverlust, sondern stellen lediglich eine Transferzahlung zwischen Steuerzahlern und Zinsempfängern dar. Bei einer Auslandsverschuldung schmälern die Zinsen- und Tilgungszahlungen die Konsummöglichkeiten zukünftiger Generationen, was eine reale Last darstellt. Somit gilt in diesem Falle die Analogie zwischen privater und öffentlicher Verschuldung. Lerner (1948) faßt die These zusammen:

„A nation owing money to other nations ... is impoverished or burdened in the same kind of way as a man who owes money to other men. But this does not hold for national debt which is owed by the nation to citizens of the same nation.... 'We owe it to ourselves' ". ${ }^{76}$

\section{2) Portfolioeffekte einer staatlichen Schuldaufnahme}

Daraus jedoch wie die Verfechter dieser Sichtweise zu schließen, daß eine inländische Staatsverschuldung keine langfristigen Konsequenzen habe, blieb (und bleibt) umstritten. Zur Diskussion lieferte u. a. Modigliani (1961) einen wichtigen Beitrag, in dem er die von der Keynesianischen Analyse vernachlässigten Bestandsände-

${ }^{74}$ Die Sammelband von Ferguson (1964) gibt einen Überblick der wesentlichen Beiträge zur Lastdiskussion.

${ }^{75}$ Modigliani (1961) faßt die Argumentation kurz zusammen, S. 109ff.

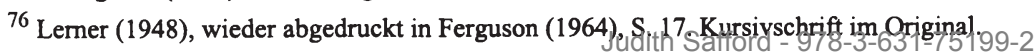


rungen in den Vordergrund stellte. Das „No-transfer No-burden“ Argument kritisierte er, weil es den produktiven Nicht-Humankapitalbestand nicht berücksichtigte. ${ }^{77}$ In einem einfachen Lebenszyklusmodell zeigt er, wie eine schuldfinanzierte Zunahme der Staatsausgaben sich negativ auf den Bestand an produktivem Kapital auswirkt. Die staatliche Bondsausgabe bietet eine zusätzliche Anlagemöglichkeit für die Ersparnisse der privaten Haushalte. Unterstellt man eine vollkommen unelastische staatliche Kapitalnachfrage, wird bei gegebenen Ersparnissen das private Kapital in den Portfolien der Haushalte in Höhe der staatlichen Bondsausgabe verdrängt. Angenommen, daß privates Kapital positive Erträge abwirft, führt die resultierende Senkung des Kapitalstocks zu einer Abnahme der gesamtwirtschaftlichen Produktion. Zukünftige Generationen werden aufgrund dieser permanenten Senkung des Kapitalstocks schlechter gestellt.

Der von Modigliani (1961) beschriebene vollständige Verdrängungsmechanismus nimmt eine diametral entgegengesetzte Position vom Ricardianischen Äquivalenztheorem ein. Das Sparen paßt sich überhaupt nicht an die erhöhte Kapitalnachfrage an, sondern ist als gegebener Anteil des Volkseinkommens exogen vorgegeben. Eine explizite Erklärung des Sparens unter Berücksichtigung seiner Zinsabhängigkeit kann zwei weitere Folgen berücksichtigen, welche die Analyse ergänzen: ${ }^{78}$ Erstens die Finanzierung der Schuldzinsen und zweitens zusätzliche Auswirkungen infolge von Faktorpreisänderungen.

\section{3) Die Finanzierung der Zinszahlungen}

Im „steady state“ Wachstum stellen die Schuldzinsen keine Last für zukünftige Generationen dar, wenn der Kapitalstock genau in Höhe der „goldenen Regel der Akkumulation" für das pareto-optimale Konsumniveau liegt [Phelps (1961)]. Dort entspricht seinem Grenzertrag (bzw. dem Zinssatz) die Wachstumsrate des Outputs. Dies läßt sich leicht wie folgt veranschaulichen.

${ }^{77}$ Er kritisierte auch die Vernachlässigung des Bestands an natürlichen Ressourcen sowie des technischen Fortschrittes, Modigliani (1961), S. 115. Letzterer wird in diesem Abschnitt unter (6) wieder aufgegriffen.

${ }^{78}$ Diese werden auf der Grundlage neoklassischer Modelle entweder des Solow-Typs oder mit überlappenden Generationen (OLG) untersucht, siehe Kitterer (1988) für eine anschauliche vergleichende Analyse. In den OLG-Modellen sind die Haushalte rational und weitsichtig. Allerdings beschränkt sich ihr Lebenshorizont im Unterschied zu Barro (1974) auf die eigenen Lebenszyklen. Der Nutzen der nachfolgenden Generation wird bei der eigenen Konsumplanung nicht berücksichtigt. Weil die neoklassische Schuldentheorie nicht von altruistischen Haushalten ausgeht, ist sie nicht mit der Neutralitätsaussage vereinbar. 
In einer vollbeschäftigten Wirtschaft wird der staatliche (pro-Kopf) Schuldenbestand (b) entsprechend dem Grenzertrag des Kapitals mit der Rate (r) verzinst. Im "steady state“ Gleichgewicht wächst die (arbeitende) Bevölkerung, der Kapitalstock, der öffentliche Schuldenbestand und der Output mit der gleichgewichtigen Rate (n). Im „steady state“ entspricht der Finanzierungsbedarf einer Periode der Differenz zwischen den gesamten fälligen Zinszahlungen ( $\mathrm{rb}$ ) und dem Beitrag der Neuverschuldung (nb). Dieser wird durch eine (pro-Kopf) Steuer (t) aufgebracht.

$$
\mathrm{t}=(\mathbf{r}-\mathrm{n}) \mathbf{b} \text {. }
$$

Ist der Kapitalstock auf dem der ,goldenen Regel“ entsprechenden effizienten Niveau, dann sind Zinssatz (r) und Wachstumsrate (n) identisch. Aus obiger Gleichung ist es klar, daß die Zinszahlungen durch die Neuverschuldung vollständig gedeckt werden und keine Steuern hierfür erhoben werden müssen. In diesem Falle stellen die Zinszahlungen keine Last für zukünftige Generationen, da sie sich aus dem Wirtschaftswachstum selbst finanzieren.

Es gibt jedoch keinen zwingenden Grund, weshalb die Sparentscheidungen entsprechend der ,goldenen Regel“ ausfällt. Ist beispielsweise $n>r$, dann wird $t$ negativ. Die Neuverschuldung deckt nicht nur die gesamten Zinszahlungen, sondern kann auch ein Primärdefizit finanzieren, beziehungsweise den (pro-Kopf) Schuldenstand senken. Dadurch nimmt das verfügbare Einkommen in jeder Periode zu und sämtliche Generationen können sich anhand der Staatsverschuldung besser stellen mit der Gewißheit, daß ihre Nachkommen nicht dafür belastet werden. Nach Meinung einiger Beobachter lag diese Situation in den 60er und 70er Jahren zeitweise vor, was die damalige Verschuldungspolitik bei sinkenden Schuldenquoten ermöglichte. ${ }^{79}$ Unterstützt durch ihre empirischen Ergebnisse bestreiten jedoch Abel et al. (1989), daß zwischen 1960-1984 der effektive Realzins auf der Staatschuld unter der Wachstumsrate lag. Ein derartiges „Ponzi-Spiel“ ${ }^{100}$ kann jedoch auf Dauer nur bei einer sehr hohen Sparquote aufrechterhalten werden, weshalb die Konstellation $n>r$ als "dynamisch ineffizient" bezeichnet wird. ${ }^{81}$ In diesem Falle ist $t>0$. Die Neuverschuldung im dynamisch effizienten Gleichgewicht reicht nicht aus, um die Zinszahlungen vollständig abzudecken und der Fehlbetrag $\mathrm{mu} ß$ anderweitig finanziert werden.

\footnotetext{
${ }^{79}$ Vgl. z. B. die Berechnungen von Rürup (1982), S. $605 \mathrm{ff}$.

${ }^{80}$ Genannt nach dem italienisch-amerikanischen Bankier, Charles Ponzi, der in den 20er Jahren Investoren sehr hohe Zinsen zahlte, welche er aus den laufenden Einnahmen von neuen Investoren finanzierte.

${ }^{81}$ Phelps (1965) weist nach, daß eine überkapitalisierte Wirtschaft, in der $\mathrm{n}>\mathrm{r}$ gilt, dynamisch ineffizient ist, weil bei gegebenem Sparen der pro-Kopf Konsumpfad unter dem optimalen Konsumpfad liegt. 


\section{4) Die Auswirkungen von Faktorpreisänderungen}

Bei den bisher abgeleiteten Ergebnissen wurden die Auswirkungen von Faktorpreisänderungen auf die Kapitalbildung vernachlässigt. Mit dieser Vereinfachung konnte gezeigt werden, da $\beta$ die negativen Auswirkungen der Staatsverschuldung nicht von Zinsveränderungen abhängig sind. Abgesehen jedoch von Grenzfällen des Sparverhaltens, wie Modigliani (1961) und Barro (1974) unterstellen, sowie dem „Kleinländerfall“ in einer offenen Wirtschaft, ist anzunehmen, daß die erhöhte Kapitalnachfrage bei einer öffentlichen Verschuldung sich auch in Zinssteigerungen niederschlägt und damit weitere Folgen mit sich bringt.

Das Zinsniveau wird zunächst durch zwei gegenläufige Effekte bestimmt. Erstens steigt die Kapitalnachfrage infolge der staatlichen Schuldtitelausgabe. Zweitens steigt das Kapitalangebot, da für gegebene Staatsausgaben eine höhere Verschuldung gleichbedeutend mit einer Steuersenkung ist. Dies bewirkt, daß das verfügbare Einkommen und damit auch die Ersparnis steigt. In der Regel dominiert der erste Effekt, zumal wenn Steuern für die Zinszahlungen erhoben werden, welche das verfügbare Einkommen wieder schmälern.

Die genauen Auswirkungen hängen jedoch davon ab, wie die Last der Zinszahlungen auf die Steuerzahler verteilt wird und wie dies wiederum ihre Ersparnisse beeinflußt. Werden spätere Generationen für die Zinssteuer herangezogen, nehmen die Ersparnisse aufgrund der Senkung ihres verfügbaren Einkommens weiter ab. Der unter Abschnitt (2) beschriebene Verdrängungseffekt wird durch diesen Zinssteuer-Effekt noch verstärkt. Wird die Zinssteuer den Nutznießern der Staatsverschuldung aufgebürdet, wird diese Last antizipiert und die Ersparnisse werden etwas steigen. Dadurch wird der Verdrängungseffekt teilweise kompensiert. Dieser positive Effekt reicht jedoch im dynamisch effizienten Gleichgewicht nicht aus, um die Sparlücke zu schließen. Die Kapitalakkumulation wird verlangsamt durch die Staatsverschuldung und infolgedessen sinkt das zukünftige Einkommen. ${ }^{82}$

Neben dieser Verlangsamung der privaten Kapitalakkumulation, steigt infolge der Neuverschuldung der öffentliche Schuldenbestand, woraus sich zusätzliche Auswirkungen ergeben. Zusammen mit dem Zinsanstieg läßt ein höherer Schuldenbestand die öffentlichen Zinszahlungen anwachsen. Setzt sich diese Entwicklung fort, wird eine kritische Finanzierungsgrenze erreicht, wo die Schuldzinsen nicht mehr wie bisher durch das Steueraufkommen zu finanzieren sind. Der Staat verschuldet sich dann, nur um seinen Zinsverpflichtungen nachzukommen. Ohne Stabilisierungsmaßnahmen steigt die öffentliche Verschuldung bis die privaten Ersparnisse komplett absorbiert werden und die private Kapitalbildung vollständig verdrängt wird.

82 Atkinson und Stiglitz (1980), S. 254 oder, im OLG-Mgdell aush Kitterer (1988), S 36 6lf-2 
Die Stärke dieser negativen Auswirkungen bzw. die Entwicklungsdynamik der Verschuldungslast hängt ab von der Höhe der Sparquote, der Primärausgabenquote, der Produktionstechnologie, bzw. der Kapitalelastizität des Outputs sowie von den anfänglichen Anteilen der Steuern und der Neuverschuldung bei der staatlichen Ausgabenfinanzierung. Eine niedrige Sparquote, hohe Staatsausgaben und hohe Kapitalelastizität in der Produktion beschleunigen den dynamischen Entwicklungsprozeß, bzw. verstärken die negativen Auswirkungen. ${ }^{83}$

\section{5) Staatsverschuldung in der offenen Wirtschaft ${ }^{84}$}

Gerade für die Analyse des Vereinigten Königreichs sind die Auswirkungen von Budgetdefiziten in der offenen Volkswirtschaft wichtig. Für ein relativ kleines offenes Land wie das Vereinigte Königreich ist das Zinsniveau weitgehend exogen durch den Weltzins vorgegeben. Unter dieser Bedingung kann ein derartiges Land sich beliebig verschulden, bzw. Kredite vergeben: Das Sparangebot ist vollkommen elastisch. Somit fallen die oben beschriebenen Verdrängungs- und Faktorpreiseffekte weg, und weder die private inländische Ersparnis noch die öffentliche Schuldaufnahme haben einen direkten Einfluß auf die Kapitalbildung oder die Produktion..$^{85}$

Negative Auswirkungen der Verschuldung folgen allerdings aus der Last der Zinszahlungen, welche das verfügbare Einkommen mindert. Dabei gelten die obigen Zusammenhänge bezüglich dem Verhältnis zwischen der Wachstumsrate (n) und dem exogenen Zinssatz ( $r$ ). Im dynamisch effizienten Fall $(r>n)$ reicht die wachstumsbedingte Neuverschuldung nicht aus, um die Zinszahlungen auf der öffentlichen Schuld vollständig abzudecken.

Die Staatsverschuldung hat auch Auswirkungen auf die Zahlungsbilanz. Die zusätzliche staatliche Kreditaufnahme verursacht einen Kapitalnachfrageüberhang, welcher durch Kapitalimporte abgebaut wird: Die Auslandsverschuldung steigt. Spiegelbildlich stellen die Zinszahlungen auf die Auslandsschuld eine Last auf die Kapitalbilanz dar. Eine kritische Verschuldungsquote existiert, wo sich diese zwei

${ }^{83}$ Siehe z. B. Carlberg (1988), S. 62ff. für ausführliche Beschreibung, formale Darstellung und numerische Fallbeispiele bei unterschiedlichen Parameterwerten des Zusammenspiels der verschiedenen Wirkungsweisen der Staatsverschuldung im neoklassischen Modell.

${ }^{84}$ Hierzu Persson (1985).

${ }^{85}$ Feldstein und Horioka (1980) fanden allerdings einen starken Einfluß des privaten inländischen Ersparnisses auf die inländische Kapitalbildung und schlossen daraus, daß die internationale Kapitalmobilität in der Realität gering sei. Folglich wären Verdrängungseffekte der Staatsverschuldung auch in einer kleinen offenen Wirtschaft zu erwarten Hierzu auch Sinn_(1992). 199-2 
Effekte genau ausgleichen. Liegt die Verschuldungsquote über diesem Niveau, dann ist der Netto-Zufluß von Auslandskapital mit einem Aufwertungseffekt verbunden. ${ }^{86}$ Von kurzfristigen Reaktionen der Leistungsbilanz abgesehen, wirkt dies tendenziell exporthemmend und importfördernd. Die Leistungsbilanz verschlechtert sich. Deswegen wird von einem „Crowding out" der Inlandsproduktion durch den Außenbeitrag gesprochen, ${ }^{87}$ welches der Hintergrund der oft hergestellten Verbindung zwischen den „Zwillings-Defiziten“ des Staates und der Leistungsbilanz darstellt.

\section{6) Öffentliche Kapitalbildung und Wirtschaftswachstum}

Die bisherigen Überlegungen knüpften an die Auswirkungen der Staatsverschuldung auf die private Kapitalbildung an. Dabei wurde der von Modigliani (1961) erwähnte Aspekt vernachlässigt, daß bei Bildung von öffentlichem Kapital durch Staatsverschuldung, sich diese produktiv im Wachstumsproze $B$ auswirken kann. Beiträge der endogenen Wachstumstheorie zeigen, wie Investitionen in öffentliche Infrastruktur, Humankapital oder Forschung und Entwicklung positive Externalitäten verursachen können, die sich auf den technischen Fortschritt im Produktionsprozeß auswirken [Barro und Sala-i-Martin (1995)]. Auch wenn die Auswirkungen von öffentlicher Kapitalbildung auf die Produktivität des privaten Kapitals und somit auf den Wachstumsprozeß sowohl theoretisch als auch empirisch umstritten sind ${ }^{88}$ machen diese neueren Theorien jedoch darauf aufmerksam, daß die öffentliche Verwendung von volkswirtschaftlichen Ressourcen und nicht lediglich ihr Anteil daran den Wohlstand gegenwärtiger und zukünftiger Generationen beeinflussen.

${ }^{86}$ Dieser Wirkungszusammenhang wurde durch die Devisenkursentwicklungen 1994 und 1995 nur teilweise bestätigt. Beispielsweise werden die Abwertungen von US\$ und Lire oft in Zusammenhang mit ihren hohen Defiziten gebracht. Möglicherweise verlangen Investoren eine Risikoprämie für eine Kapitalanlage, die mit steigenden Defiziten als zunehmend riskant eingestuft wird. Der Ausgleich findet nicht allein über Zinsdifferentiale statt, sondern auch über den Wechselkurs. Dieser Abwertungseffekt überwiegt den langfristigen Aufwertungseffekt, IMF (1995b), S. 36f.

${ }^{87}$ Vgl. etwa Gandenberger (1981), S. 22

88 Siehe Ott (1993), S. 266ff. für einen kurzen Überblick und eine Diskussion der Theorien zur öffentlichen Kapitalbildung und Wirtschaftswachstum. 


\section{Wirkungen der Staatsverschuldung auf die Einkommensverteilung}

Bei der bisherigen Diskussion der drei Theorien der Staatsverschuldung wurde auf die Verteilungswirkungen der Staatsverschuldung selbst nur wenig eingegangen. Es dürfte jedoch klar sein, daß die Finanzierung einer gegebenen staatlichen Ausgabenhöhe untrennbar mit der Verteilung des verfügbaren Einkommens verbunden ist. Ziel dieses Kapitels ist es, diesen Zusammenhang ausführlicher zu begründen und damit die Verteilungseffekte ${ }^{89}$ der Staatsverschuldung zu erläutern.

In den folgenden ersten zwei Abschnitten werden die Verteilungswirkungen der herkömmlichen Theorien kurz erläutert. In der neoklassischen Analyse stehen die intertemporalen Verteilungswirkungen der Staatsverschuldung (z. B. zwischen Generationen) im Vordergrund. Diese Theorie wird vervollständigt durch den ,generational accounts"-Ansatz. Sowohl bei der neoklassischen als auch der Keynesianischen Staatsschuldentheorie hat eine staatliche Kreditaufnahme aber auch Folgen auf die Einkommensverteilung zwischen Individuen oder gesellschaftlichen Gruppen innerhalb einer Periode. Ergänzend zu diesen bekannten Ergebnissen werden dann im Abschnitt (3) einige der neueren polit-ökonomischen Ansätze zur Erklärung der Staatsverschuldung untersucht.

\section{1) Intertemporale Wirkungen}

\section{a) Intertemporale Wirkungen nach der herkömmlichen Schuldentheorie}

In einer geschlossenen Wirtschaft sind entsprechend der Logik des „Transfer“ Ansatzes intertemporale Verteilungswirkungen der Staatsverschuldung ausgeschlossen. Grund hierfür ist die implizite Bezugnahme auf den gegenwärtigen Konsum und die Investitionen als Bestandteile der Keynesianischen Einkommensgleichung, von denen ein intertemporaler Ressourcenentzug nicht möglich ist. Die Investitionen haben jedoch eine Doppelfunktion, welche bei dieser Sichtweise vernachlässigt wird: Sie sind Bestimmungsgröße nicht nur des gegenwärtigen, sondern auch des zukünftigen Einkommens, weil die Nettoinvestitionen den Zuwachs des volkswirtschaftlichen Kapitalstocks darstellen. Gerade aus dieser zweiten Funktion leitet sich die zentrale Aussage der neoklassischen Theorie über die intertemporale Wirkung der Staatsverschuldung ab. Kreditfinanzierte Staatsausgaben erhöhen zwar

${ }^{89}$ Dabei wird von der Vermögensverteilung abgesehen; es werden lediglich die Einkommensverteilungseffekte untersucht. 
das verfügbare Einkommen der steuerlich entlasteten Generation in der Periode der Schuldenaufnahme: Die vom Staat beanspruchten Ressourcen werden jedoch dem Kapitalmarkt entzogen und verdrängen die private Investitionstätigkeit. Deshalb hat eine Kreditfinanzierung einen kleineren, privaten Kapitalstock in der Zukunft als Folge als dies bei einer Steuerfinanzierung der Fall gewesen wäre. Das Wachstum des Produktionspotentials fällt geringer aus, was eine Senkung des künftigen Realeinkommens für alle zukünftigen Generationen zur Folge hat. Hierin liegt nach der neoklassischen Ansicht die intertemporale Verteilungswirkung der Staatsverschuldung.

Bei ihren Beurteilungen einer staatlichen Kreditaufnahme in einer vollkommen offenen, kleinen Volkswirtschaft unterscheiden sich der Transfer Ansatz und die neoklassische Sichtweise nicht wesentlich. Die Last besteht in den Zins- und eventuellen Tilgungskosten in zukünftigen Perioden, wenn davon ausgegangen wird, daß diese Beträge durch die zukünftigen Steuerzahler finanziert werden und dann ins Ausland abfließen. Somit verursacht die Kreditfinanzierung der Staatsausgaben im Ausland ebenfalls einen Ressourcenentzug, der zukünftige Generationen belastet.

\section{b) Intertemporale Effekte nach dem „Generational Accounting“ Ansatz}

Bei den bisherigen Ansätzen wurde als Indikator für die Fiskalpolitik und damit für das Ausmaß der Umverteilung zwischen den Generationen das jährliche Defizit herangezogen. Dieses Konzept spiegelt jedoch nur die unmittelbare Nachfragesteuerung der Fiskalpolitik wider und ist deswegen nicht dazu geeignet, die intertemporalen Verteilungswirkungen fiskalpolitischer Aktivität zu erfassen. ${ }^{90}$ Die Konsum- und Sparentscheidungen, welche die intertemporalen Lastverschiebungen verursachen, basieren nicht allein auf den Strömen der jährlichen Einnahmen und Ausgaben im Haushaltsdefizit. Konsumentscheidungen werden in Abhängigkeit von den verfügbaren Ressourcen über die ganze Lebenszeit hinweg gefällt. Dies zu berücksichtigen, ist auch die Absicht langfristig ausgelegter Modelle, wie beispielsweise in der neoklassischen Theorie. Deswegen wäre es angebracht, für die langfristigen Wirkungen der Fiskalpolitik ein Konzept zu entwickeln, welches das Ausmaß der staatlichen Umverteilung über den gesamten Lebenszyklus für gegenwärtige und zukünftige Generationen mißt.

Mit dem Ansatz des „generational accounting“ wurde ein derartiges Konzept entwickelt. Wie der Name andeutet, besteht das Grundprinzip von „generational accounting“ darin, eine intergenerationale Bilanz aufzustellen und die Inzidenz von

${ }^{90}$ Zur Kritik der herkömmlichen Definition staatlicher Budgetierung siehe Auerbach und Kotlikoff (1987), S. 103ff. 
staatlichen Transferzahlungen und Steuern auf verschiedene Altersgruppen bzw. Generationen zu quantifizieren. Ein Schlüsselbegriff dabei ist die NettoSteuerzahlung, welche als Steuerzahlungen abzüglich erhaltener Transferzahlungen definiert ist. ${ }^{91}$ Der Gegenwartswert von erwarteten Transferzahlungen über die gesamte Lebenszeit hinweg - d. h. für Pensionen, Krankheits- und Arbeitsentschädigung, etc. - wird dem Gegenwartswert der gesamten Steuerzahlungen über die Lebenszeit - z. B. Einkommensteuer, soziale Beiträge und Vermögenssteuer - gegenübergestellt.

Das Verhältnis von Steuern zu erhaltenen Leistungen für jede Generation gibt Auskunft über die Umverteilungen zwischen den Generationen bei einem gegebenen Steuer- und Transfersystem. Dies ermöglicht eine Beurteilung der langfristigen Tragbarkeit einer gegebenen Politik, was bei der erwarteten Überalterung der Bevölkerung besonders notwendig ist..$^{92}$ Verschiedene Länderstudien haben gezeigt, ${ }^{93}$ $\mathrm{da} ß$ bei jeweils unveränderter Politik große Unterschiede in den NettoSteuerzahlungen zwischen verschiedene Generationen bestehen können. Die Ergebnisse derartiger Studien legen offen, daß bei den meisten gegenwärtigen Systemen die Transferzahlungen zu hoch und langfristig politisch nicht tragbar sind. ${ }^{94}$ Daraus wäre zu schließen, daß die jüngeren Generationen, vor die Perspektive gestellt, Nettozahler zu sein, politischen Druck für eine Senkung der Transferzahlungen ausüben müßten.

„Generational accounts“ hängen von einigen wichtigen langfristigen Annahmen ab, wie z. B. der zukünftigen Produktivität, dem Bevölkerungswachstum und damit dem zukünftigen Wachstum, sowie von der realen Diskontierungsrate der Steuerzahler. Deswegen sind ihre Prognosen mit einer gewissen Willkür behaftet. Trotzdem nimmt das "generational accounting“ im Unterschied zum gegenwärtigen Budgetdefizit eine langfristige Perspektive ein und bietet somit eine nützliche Grundlage für die Diskussion über die zukünftigen Gewinner und Verlierer der Fiskalpolitik.

\footnotetext{
91 Auerbach et al. (1991), S. $2 \mathrm{ff}$.

92 Boll et al. (1994).

${ }^{93}$ Vgl. z. B. Auerbach et al. (1991) für die U.S.A. und Gokhale et al. (1995) für die Bundesrepublik Deutschland.

${ }^{94}$ Dabei schneidet das Vereinigte Königreich relativ gytad ab vgl, za B B Hills (1996) 


\section{2) Intratemporale Verteilungswirkungen}

\section{a) Interpersonelle Verteilungswirkungen im Transferansatz}

In der Gleichgewichtsanalyse bei Vollbeschäftigung ${ }^{95}$ hat die Staatsverschuldung neben ihrem Einfluß auf die zeitliche Lastverteilung ebenfalls Auswirkungen auf die Einkommensverteilung im herkömmlichen Sinne, d. h. auf die Verteilung zwischen Individuen. Auch heute findet sich in der Diskussion die zunächst plausibel lautende Auffassung, daß die Verteilungseffekte in erster Linie von der Verteilung der Staatsgläubiger und der Zinssteuerzahler abhängen. ${ }^{96}$ Angenommen, daß Steuereinnahmen die Zinszahlungen der Staatsschuld finanzieren, wird einerseits den Zinssteuerzahlern verfügbares Einkommen entzogen und andererseits den Zinsempfängern zusätzliches Einkommen überwiesen. Zwischen diesen zwei Gruppen findet eine Umverteilung statt, es sei denn, daß sie miteinander identisch sind. Die Zinssteuer kann jedoch nicht ausschließlich von den Zinsempfängern erhoben werden, weil die Nettoverzinsung der Staatsanleihen dann gleich Null wäre, was sie unverkäuflich machte. Wenn die Inhaber der Staatsschuldpapiere zur oberen oder zumindest mittleren Einkommensgruppen gehören, dagegen die Zinssteuerzahler allen oder zumindest teilweise den unteren Einkommensschichten zugeordnet werden, dann findet eine Verteilung ,von unten nach oben“ statt.

\section{b) Funktionelle Verteilungswirkungen im neoklassischen Ansatz}

In Frage gestellt wurde der Transferansatz jedoch u. a. von Gandenberger (1970), der die darin hergestellte Kausalität zwischen dem Zinseinkommen der Staatsgläubiger und der Erhebung der Zinssteuer bestritt: Das Zinseinkommen sei keine Folge der Zinssteuer, sondern einer von der Staatsverschuldung unabhängigen Sparentscheidung. Die Sparer profitieren nur dann von der Anleihefinanzierung, wenn diese unmittelbar eine Veränderung der Kreditmarktbedingungen - insbesondere einen Zinsanstieg - verursachen. Somit mündet die Frage nach den intratemporalen Verteilungswirkungen der Staatsverschuldung in eine Analyse der gesamten Kapitalmarktreaktion auf die Anleihenausgabe. Wenn sich die Zinssätze infolge der staatlichen Schuldaufnahme erhöhen, trifft dies nicht nur die Zeichner von Staatsanleihen, sondern auch die Besitzer von allen anderen verzinsten Kapitalanlagen, sowie von Realvermögen, deren zukünftige Erträge mit einem höheren Zinssatz abdiskontiert werden müssen. In einer umfassenden Analyse der intratemporalen Verteilungswirkungen der Staatsverschuldung müßten deshalb nicht nur die Folgen

\footnotetext{
${ }^{95}$ Auf die Analyse der Verteilungswirkungen von konjunkturpolitisch motivierter Verschuldungspolitik soll hier nicht eingegangen; siehe hierzu z. B. Oberhauser (1970).

${ }^{96}$ Siehe z. B. Michl (1990).
} 
für die gesamte Kapitalbildung in einer Volkswirtschaft, sondern auch der Einfluß auf die Faktorpreisverhältnisse unter Berücksichtigung der Grenzproduktivitätstheorie untersucht werden. So geht die Analyse der intratemporalen Verteilungswirkungen der Staatsverschuldung von der Betrachtung einer interpersonellen Verteilung zwischen „reich“ und „arm“ in eine funktionale Verteilungsanalyse der Besitzer der Produktionsfaktoren Kapital und Arbeit über.

Wie bereits unter Abschnitt III.4 erläutert, ist in einer geschlossen Wirtschaft anzunehmen, daß ein erhöhter Kreditbedarf infolge einer staatlichen Schuldaufnahme Zinssatzsteigerungen auslöst. Die Kapitalintensität nimmt ab, was sinkende Bruttolöhne zur Folge hat. Unter Berücksichtigung einer eventuellen Besteuerung der Produktionsfaktoren werden die Auswirkungen des Zinssatzanstiegs etwas gedämpft, dagegen die Lohnsenkung verstärkt. ${ }^{97}$

\section{c) Intratemporale Verteilungswirkungen in der heutigen Diskussion}

Die oben beschriebenen Ergebnisse wurden bereits in den 60er und 70er Jahren abgeleitet. ${ }^{98}$ Seitdem wurden die intratemporalen Auswirkungen der Staatsverschuldung bis auf einige polit-ökonomische Beiträge, die noch vorgestellt werden, kaum noch in der Literatur beachtet. Dies dürfte zum einen daran liegen, daß die Verteilungseffekte in Folge von Faktorpreisänderungen in einer „kleinen“, offenen Volkswirtschaft mit integrierten internationalen Kapitalmärkten weitgehend entfallen. Wie bereits erläutert, bedeutet der unbeschränkte Kapitalimport für das betroffene Land eine elastische Kapitalangebotsfunktion. Das Weltzinsniveau stellt sich ein und der Zinseffekt infolge einer Staatsschuldausgabe unterbleibt.

Zum zweiten ist die empirische Relevanz der Staatsverschuldung für die Einkommensverteilung kaum abzuschätzen. ${ }^{99}$ Über die Gläubigerstruktur der Staatsschuld, insbesondere über den personellen Besitzanteil durch die finanzielle Intermediation von Pensionfonds und Versicherungen, ist zu wenig bekannt, um eine empirische Überprüfung zu ermöglichen. ${ }^{100}$ Neben der Unmöglichkeit, die Zinsempfänger für empirische Zwecke zu bestimmen, müßten für eine Überprüfung der postulierten funktionalen Verteilungswirkungen die Auswirkungen der Staatsverschuldung auf Zinssätze und Kreditmenge ermittelt werden können. Wie bereits bei der Diskussion um das Ricardianischen Äquivalenztheorem erwähnt, liefern auch neuere empi-

${ }^{97}$ Carlberg (1988), S. 58f.

${ }^{98}$ Siehe z. B. Diamond (1965) und Gandenberger (1970).

${ }^{99}$ Ausführlich hierzu siehe Gandenberger (1970), S 39.

100 Siehe z. B. die Statistik der Bank of England im Quarterly Bulletin (1995), S. 351. Einen „heroischen" Versuch über die Verteilung der Staatsschuld in den U.S.A. macht jedoch Michl (1990). 
rische Ergebnisse diesbezüglich keine unumstrittenen Ergebnisse. Angesichts des Schweigens in der Literatur scheint die Schlußfolgerung von Gandenberger (1970, S. 41) heute noch gültig, der schreibt:

„... beim derzeitigen Stande der Wissenschaft [ist] ein klares Urteil über die Wirkungen der Kreditfinanzierung auf die funktionelle und personelle Einkommensverteilung nahezu unmöglich.“

\section{3) Polit-ökonomische Ansätze zur Verteilung und Staatsverschuldung}

Normative Schuldentheorien gehen in der Regel davon aus, daß ökonomische Entscheidungen von einem „benevolenten Diktator" durch „erstbeste“ Politikstrategien bestimmt werden, deren Ziel die Wohlstandsmaximierung eines repräsentativen Individuums unter gegebenen Beschränkungen ist. ${ }^{101}$ Abweichungen von derartigen Politikstrategien, wie z. B. der verbreitete Anstieg der Staatsverschuldung der letzten Jahrzehnte, können diese Theorien nicht erklären. Genauso wenig vermögen sie die sehr unterschiedlichen Entwicklungen der Staatsverschuldung der aus ökonomischer Sicht relativ homogenen OECD-Ländern begründen. An diesen zwei Beobachtungen setzen polit-ökonomische Erklärungen der Staatsverschuldung an. Sie versuchen die Bedeutung von politischen und institutionellen Merkmalen bei wirtschaftspolitischen Entscheidungen zu erfassen, wie z. B. Verteilungskämpfe, ideologisches und opportunistisches Politikerverhalten, Gesetzgebung und Budgetregeln. Im Unterschied zur „Public Choice“ Theorie wird jedoch von rationalen Erwartungen und optimierendem Verhalten der beteiligten Individuen ausgegangen. ${ }^{102}$ Bei den verteilungspolitischen Modellen wird die Bedeutung der Einkommens- und manchmal auch der Vermögensverteilung als politisches Moment untersucht. Diese drücken sich in politischen Entscheidungen aus, die entweder zu intertemporalen Umverteilungen oder Verteilungskonflikten zwischen sozialen Gruppen und/oder politischen Parteien führen.

\section{a) Intergenerationale Umverteilungen}

Aus politischer Sicht sind intergenerationale Verteilungswirkungen der Staatsverschuldung möglich und naheliegend, weil die gegenwärtige Generation allein über

\footnotetext{
101 Als normative Meßlatte einer optimalen Staatsschuldenentwicklung wird in der Literatur z. B. der „Tax Smoothing“ Ansatz von Barro (1979) herangezogen, vgl. z. B. Roubini, N. und Sachs, J. (1989a) oder Alesina und Perotti (1994). Dieser Theorie zufolge können Budgetdefizite die Auswirkungen von exogenen Schocks dämpfen, weil sie die staatliche Einnahmehöhe stetig halten.

102 Einen Überblick dieser Theorien bietet Alesina (1992)
} 
die Schuldenpolitik abstimmt. Folglich kann sie sich für eine Fiskalpolitik entscheiden, welche ihre Nachkommen belastet.

Cukierman und Meltzer (1989) haben diesen Gedanken in einem Modell mit überlappender Generationenstruktur (OLG-Modell) untersucht, in dem alle von Barros Prämissen außer derjeniger vollkommener Kapitalmärkte gelten. Es gibt eine alte und eine junge Generation in einer stabilen Bevölkerung. Die Staatsausgaben bestehen aus Schuldzinsen und Transferzahlungen an die Alten und werden durch eine Pauschalsteuer auf die junge Generation und die Schuldaufnahme finanziert. Unterschiedliche Fähigkeiten unter der jeweiligen Generation führen zu unterschiedlichen Arbeitserträgen und Vermögensbeständen und somit zu „reichen“ und „armen“ Individuen. Erstere sind zunächst einmal indifferent gegenüber der Schuldenpolitik, da sie positive Erbschaften hinterlassen und damit intertemporale Steuerverschiebungen kompensieren können: Für sie gilt die Ricardianische Äquivalenz. Letztere sind liquiditätsbeschränkt aufgrund unvollkommener Kapitalmärkte und möchten negative Erbschaften hinterlassen. Da dies nicht möglich ist, ${ }^{103}$ stimmen sie für eine staatliche Verschuldung, welche ihre Konsummöglichkeiten erhöht. Aus dieser Konstellation führt die soziale Wahl im Mehrheitswahlsystem zur Schuldenaufnahme und zur intertemporalen Umverteilung. Im geschlossenen neoklassischen Gleichgewichtsmodell wird der Konsumanstieg der beschränkten Individuen von den nicht-beschränkten Individuen finanziert, welche bei der Schuldausgabe Bonds für Kapital ersetzen. Es kommt folglich zu einer Verdrängung des Kapitals („Crowding-Out“) und einer Veränderung des Faktorpreisverhältnisses, d.h. einer Senkung der Kapitalintensität. Alle Gesellschaftsmitglieder (d. h. auch die „Reichen“) werden von diesen Faktorpreisveränderungen getroffen, was die ursprünglichen Präferenzen bezüglich der Fiskalpolitik und folglich das Wahlverhalten der einzelnen Individuen verändert.

Cukierman und Meltzer (1989) untersuchen die ökonomischen Bedingungen, die in diesem Szenario zu erhöhten Defiziten und Staatsverschuldung führen. Sie zeigen, daß die Staatsverschuldung um so größer wird:

a) je größer das erwartete Wirtschaftswachstum ist, weil unter den angenommenen Produktionsbedingungen arbeitssparender technischer Fortschritt den Anteil liquiditätsbeschränkter Individuen erhöht;

b) je größer der Anteil von Gesellschaftsmitgliedern ist, dessen Einkommen und Vermögen (aus Sach- und Humankapital) eine kritische Grenze unterschreitet, weil diese die Aufhebung der Liquiditätsbeschränkung wünschen;

${ }^{103}$ Vgl. Fußnote 18. 
c) je kleiner der Gesellschaftsanteil ist, der sein Einkommen vorwiegend aus dem Faktor Arbeit bezieht, weil diese Gruppe aufgrund der Arbeitsertragseinbuße gegen die Verschuldung ist;

d) je breiter die Streuung der Einkommens- und Vermögensverteilung ist, weil Verschuldung von den Extrempolen der Verteilung bevorzugt wird [b) und c)];

e) je empfindlicher der Kapitalertrag und je unempfindlicher der Arbeitsertrag auf eine Veränderung der Kapitalintensität reagiert;

f) je länger die Lebenserwartung, weil dann der Nutzen des Konsums im Alter und damit die Wahrscheinlichkeit einer Liquiditätsbeschränkung zunimmt.

Interessant bei diesem Modell ist die implizite Wechselwirkung zwischen Staatsverschuldung und ihren Verteilungswirkungen. Die Staatsverschuldung begünstigt die Empfänger von Kapital- und Transfereinkommen auf Kosten der Lohnempfänger. Diese Verschiebungen zwischen den beiden gesellschaftlichen Gruppen führen dann zu einer verstärkten Lobby für die Staatsverschuldung und für die intertemporale Lastverschiebung. ${ }^{104}$ In der Tat ging der verbreitete Anstieg der Staatsverschuldung seit den 70er Jahren häufig mit einer Verschiebung bei den Staatsausgaben von öffentlichem Konsum und Investitionen zu erhöhten Zinszahlungen und Transferzahlungen einher. ${ }^{105}$ Nach Cukierman und Meltzer (1989) hatten die Interessen der Kapital- und Transferempfängergruppen eine positive Wirkung auf die Schuldenentwicklung.

Gegen die Hypothese von Cukierman und Meltzer (1989) läßt sich einwenden, daß sie in erster Linie auf die intertemporale Verteilung als Bestimmungsparameter für die Staatsverschuldung baut. Die öffentliche Verschuldung ist jedoch häufig innerhalb einer kurzen Zeit akkumuliert und wieder abgebaut worden, d. h. innerhalb einer Generation (Poterba und Summers 1986, Alesina 1988), was intertemporale Verteilungseffekte reduziert. Ferner wird im Modell nicht erklärt, warum die Zahlungsversprechungen der Schuldzinsen und Tilgung von der nachfolgenden Generation eingehalten werden. Cukierman und Meltzer (1989) nehmen an, daß private

${ }^{104}$ Die komplette Aufhebung der Liquiditätsbeschränkung für alle Individuen durch zusätzliche Verschuldung ist jedoch unmöglich, weil der negative Effekt des fallenden Arbeitsertrags dem positiven Konsumeffekt der Verschuldung auf die privaten Budgetbeschränkungen entgegenwirkt. Der geschlossene neoklassische Modellrahmen bedeutet ohnehin, daß der Staatsverschuldung durch Verdrängung des Kapitals Grenzen gesetzt ist, da ungebremste Neuverschuldung letztendlich zur vollständigen Verdrängung des Kapitals führt, vgl. Abschnitt III.4).

${ }^{105}$ Diese bekannte Entwicklung wird z. B. von Roubini und Sachs (1989b), S, 105ff dokumentiert. 
Schulden nicht vererbt werden können. Warum dann können staatliche Schulden vererbt werden? ${ }^{106}$

Tabellini (1991) begegnet dieser Kritik durch den expliziten Einbezug von intragenerationalen Verteilungskonflikten. Eine Zahlungsverweigerung verteilt von Schuldinhabern zu Steuerzahlern um, d. h. von der ,alten“ zu der ,jungen“ Generation, sowie von den „Reichen“ (die Besitzer von Staatspapieren) zu den „Armen“. Nach Tabellini (1991) stimmt ein „reicher“, junger Steuerzahler gegen eine Zahlungsverweigerung, weil er um die Wohlfahrt seine „reichen“ Eltern besorgt ist. Folglich enthält die Wahlgruppe, welche der Staatsverschuldung und ihrer späteren Zahlung zustimmen, auch Mitglieder der jungen Generation. Tabellini (1991) zeigt, wie unter bestimmten Bedingungen das politische Gleichgewicht die Schuldenausgabe und ihre Gewährung zur Folge haben. Sein Beitrag lenkt die Aufmerksamkeit auf die polit-ökonomische Bedeutung intragenerationaler Verteilungskonflikte bei den Staatsschulden.

\section{b) Intragenerationale Verteilungskonflikte}

Nach dem Grundgedanken dieser Ansätze ist die Wahl der staatlichen Schuldenpolitik das Ergebnis eines Verteilungskampfes zwischen verschiedenen ökonomischen Akteuren innerhalb einer Generation, welches sich im budgetpolitischen Verhalten der Regierung widerspiegelt. Dabei gibt es zwei Modelltypen. Im ersten werden politische Spiele zwischen abwechselnden Regierungsparteien, den Mitgliedern einer regierenden Koalition oder deren Wähler untersucht, die um die Machterhaltung kämpfen. ${ }^{107}$ Der Konflikt wird als spieltheoretische Interaktion im Zeitverlauf dargestellt. Ausschlaggebend für die Handlungen ist die Einschätzung der Zukunft der Machtinhaber (d. h. wie sie die Zeit diskontieren). Ist ihre Situation politisch sehr instabil, dann erwarten sie nicht, die späteren Kosten einer staatlichen Schuldenaufnahme tragen zu müssen und wählen die Staatsverschuldung, um ihre Amtszeit zu verlängern. Diese Art von Instabilität ist typisch in Ländern mit dem Verhältniswahlsystem, weil dieses in der Regel zu unklaren Machtverhältnissen in der Regierung, wie z. B. Koalitionen, führt. ${ }^{108}$ Im Vereinigten Königreich wird jedoch die Regierung nach dem Mehrheitwahlsystem gewählt und die Konservative Regierung ist seit 1979 mit einer absoluten Mehrheit in beiden Parlementshäusern im Amt. Diese Ansätze können allenfalls dahingehend interpretiert

106 Oder umgekehrt, warum nutzt die gegenwärtige Generation diese Versprechungen nicht vollständig aus und finanziert die Staatsausgaben allein durch Verschuldung? Alesina und Perotti (1994), S. 15 sowie van Velthoven, et al. (1993), S. 28.

${ }^{107}$ Persson und Svensson (1989), Alesina und Tabellini (1990), Tabellini und Alesina (1990), Aghion und Bolton (1990), Roubini und Sachs (1989a,b).

${ }^{108}$ Grilli et al. (1991), S. 350ff. 
werden, $\mathrm{da} \beta$ die Konservative Regierung mit dem Mehrheitwahlsystem den Ausdruck von Verteilungskonflikten in finanzpolitischen Entscheidungen effektiver unterbinden konnte. Sie werden im folgenden nicht weiter beachtet.

In der zweiten Modellgruppe kämpfen die Vertreter unterschiedlicher Interessenoder Gesellschaftsgruppen um die Budgetgestaltung. ${ }^{109}$ In diesem Falle wird die Budgetpolitik nicht durch strategische Überlegungen bestimmt, sondern sie stellt unterschiedliche und vereinzelte Ausgaben- und Steuerentscheidungen dar, welche sich aus der Unfähigkeit zur Veränderung des Status Quos ergeben. ${ }^{110}$ Einer schwachen Regierung gelingt es nicht, sich im Verteilungskonflikt durchzusetzen. Politisch notwendige, aber unbeliebte Budgetentscheidungen wie die Kürzungen von Ausgaben oder Steuererhöhungen werden verschoben. Die Wahl der Schuldenaufnahme verbleibt als Ausweichstrategie. Bei dieser Hypothese geht es darum, wie die Einflußnahme auf wirtschaftspolitische Entscheidungen zu einem gegebenen Zeitpunkt geschieht, ${ }^{111}$ und inwiefern Entscheidungsschwäche der Regierung die Kreditfinanzierung fördert.

Regierungsschwäche ist ein vages Konzept, das sich nicht einfach quantifizieren läßt. ${ }^{112}$ Ähnlich wie beim ersten Modelltyp spielen Regierungsform und -stabilität (die erwartete Dauer einer Regierung oder die Anzahl der Koalitionsmitglieder) eine Rolle. Aber selbst eine in diesem Sinne stabile Regierung kann sich als schwach, bzw. beschlußunfähig erweisen, wenn z. B. der interne budgetäre Entscheidungsproze $ß$ durch verteilungspolitische Konflikte beeinflußt werden kann. Defizit- und Ausgaberegeln und die Regelung der Entscheidungsprozesse für den Haushalt werden deswegen bei den empirischen Arbeiten quantifiziert und als Indikatoren für Schwäche interpretiert. ${ }^{113}$ Die Forschung auf diesem Gebiet steht leider erst in den Anfängen. ${ }^{114}$ Deshalb kann auf eine ausführliche Behandlung der Merkmale von „schwachen“ Regierungen, und darauf inwiefern die Konservative Regierung durch diese gekennzeichnet ist, in dieser Abhandlung nicht eingegangen

${ }^{109}$ Eine derartige Vorstellung schwebte Keynes (1922), S. 47ff, vor, der sich mit dem Abbau der britischen Staatsverschuldung nach dem Ersten Weltkrieg beschäftigte. Vgl. hierzu auch Alesina (1988).

${ }^{110}$ Formal wird dieser Gedanke ausgearbeitet bei Alesina und Drazen (1991) sowie Perotti (1992).

${ }^{111}$ D. h. es geht nicht um die strategische Einflußnahme im Zeitverlauf, wie dies bei der ersten Modellgruppe der Fall ist.

112 Siehe Grilli et al. (1991), S. 350ff, sowie von Hagen (1992), S. $23 \mathrm{ff}$.

${ }^{113}$ Vgl. z. B. die Arbeiten von Alesina (1988), Roubini und Sachs (1989b), Grilli et al. (1991) sowie von Hagen (1992) Alesina und Perotti (1996).

114 Die ersten umfassenden empirischen Arbeiten zum Zusammenhang zwischen den Merkmalen der politischen Institutionen und den offentlichen Finanzen sind Grilli et al. (1991) und von Hagen (1992). Für einen Überblick und Diskussion der bisherigen theoretischen Arbeiten siehe Alesina und Perotti (1996). 
werden. Auffallend für die vorliegende Arbeit ist es, daß in der Literatur das Interesse zwar den Merkmalen von „schwachen“ Regierungen gilt, die sich dem unterstellten Druck für Mehrausgaben oder Steuersenkungen beugen. Die Urheber dieses Druckes werden exogen angenommen. Ihre Identität sowie ihre Motive bleiben jedoch unklar. ${ }^{115}$ Anzunehmen ist, daß die Regierung sich nicht durch die Mehrheit sondern durch Partikularinteressen von budgetpolitischen Entscheidungen abhalten läßt. Andererseits wird an späterer Stelle gezeigt werden, daß die Bedeutung von aus politischen Gründe bewilligten staatlichen Privilegien, (sogenannte ,pork barrel projects") für den Abbau der Staatsverschuldung gering ist.

\section{Zusammenfassung des ersten Kapitels}

In diesem Kapitel werden die allokativen und distributiven Auswirkungen der Staatsverschuldung untersucht. Der ersten Abschnitt befaßt sich mit dem Theorem der Staatsschuldneutralität. Nach Darstellung des Grundgedankens zeigte die Diskussion seiner Prämissen, da $\beta$ diese zum Teil sehr restriktiv sind und kaum in allen Fällen erfüllt sein dürften. Daraus ließ sich folgern, daß die Staatsverschuldung reale Wirkungen hat und das öffentliche Interesse daran legitim ist.

Die zweiten und dritten Abschnitte stellen die zwei allgemein bekannten Schuldentheorien dar. Die Untersuchung der Keynesiansichen Begründung der Staatsverschuldung im zweiten Abschnitt zeigt, wie der Einsatz von temporären Defiziten bei Unterbeschäftigung möglicherweise, aber nicht im jeden Fall, die fehlende gesamtwirtschaftliche Nachfrage ersetzen kann. Allerdings reicht die resultierende Output-Erhöhung wahrscheinlich nicht aus, um diese Schuldaufnahme durch zusätzliche Steuereinnahmen zu finanzieren. Die Kosten der Nachfragepolitik müssen durch weitere Politikmaßnahmen aufgebracht werden.

Gegenstand des dritten Abschnitts sind die langfristigen Auswirkungen der Staatsverschuldung. Diese betreffen vor allem die Auswirkungen auf die private Kapitalbildung, welche durch die staatliche Inanspruchnahme von Finanzmitteln und den resultierenden Zinsanstieg verdrängt wird. Wenn die Kapitalbildung sinkt, fällt auch das Pro-Kopf-Einkommen geringer aus. Dieses Ergebnis wird für eine kleine offene Wirtschaft mit vollkommener Kapitalmobilität etwas modifiziert. Das Sparangebot ist nicht durch das Verhalten der Inländer begrenzt, sondern aufgrund Ka-

115 Ausnahmen sind die Modelle von Alesina (1988), sowie Alesina und Drazen (1991), welche zwischen sozioökonomischen Gruppen (d. h. Kapitalisten, Arbeiter und Rentiers) differenzieren, die unterschiedliche Präferenzen bezüglich der Schuldenpolitik äußern. Ähnlich wie bei Cukierman und Meltzer (1989) sind die „Arbeiter“ für Staatsverschuldung. 
pitalimport unendlich elastisch. Folglich verursacht die höhere Kapitalnachfrage keinen Zinsanstieg. Allerdings müssen die Inländer die Zinszahlungen auf die aufgenommene Schuld aufbringen, was einen Ressourcenentzug bedeutet.

Im vierten Abschnitt geht es um die Verteilungswirkungen der Staatsverschuldung. Zuerst werden die herkömmlichen intertemporalen und interpersonellen Verteilungswirkungen der Staatsverschuldung, sowie die Generational Accounts Ansätze dargestellt. Anschließend werden die bisherigen polit-ökonomischen Ansätze zum Zusammenhang zwischen Staatsverschuldung und Verteilung diskutiert. Politökonomische Modelle der Verteilungskonflikte, welche zur Wahl der Staatsverschuldung führen, betonen das Interesse liquiditätsbeschränkter Individuen an den erhöhten Konsummöglichkeiten durch Staatsverschuldung und wie schwache Regierungen der politischen Druck für Staatsverschuldung nachgeben. 
Judith Safford - 978-3-631-75199-2

Downloaded from PubFactory at 01/11/2019 07:12:45AM

via free access 


\section{Zweites Kapitel}

\section{ALLGEMEINER TEIL II: STRATEGIEN ZUR SENKUNG DER STAATSVERSCHULDUNG}

\section{Ein Überblick über die Konsolidierungsoptionen}

In den drei bekannten Modellen der Staatsverschuldung vom vorangegangenen Kapitel sind die fiskalpolitischen Regeln über die Finanzierung der Zinszahlungen vorgegeben: Das einzige fiskalpolitische Instrument ist eine zusätzliche Kreditaufnahme, welche eine weitere Zinslast in den folgenden Perioden zwangsläufig verursacht. Es wurde jedoch bereits deutlich, ${ }^{116} \mathrm{da} \beta$ dem Staat mehrere finanzpolitischen Optionen offen stehen, um die Zinszahlungen eines aufgenommenen Kredits zu finanzieren. Dies gilt ebenso, wenn er die Staatsschuld tilgt, wie dies im Vereinigten Königreich in den 80er Jahren geschah. In diesem Kapitel werden die unterschiedlichen staatlichen Finanzierungsquellen zunächst identifiziert und voneinander abgegrenzt. Sodann werden in den folgenden Abschnitten die theoretischen Möglichkeiten ihrer Verwendung zur Senkung der Staatsverschuldung untersucht.

\section{1) Finanzierungsquellen im Lichte der staatlichen Budgetbeschränkung}

Wir greifen die bereits im ersten Kapitel dargestellte staatliche Budgetrestriktion wieder auf:

$$
P_{t} G_{t}+T r_{t}-T_{t}+\left(1+i_{t-1}\right) B_{t-1}-\left(M_{t}-M_{t-1}\right)-\left(A_{t}-A_{t-1}\right)=B_{t}
$$

Mit einer einfachen Umformulierung läßt sie sich in Ausgabe- und Einnahmeposten aufteilen:

$$
P_{t} G_{t}+T r_{t}+i_{t-1} B_{t-1}=T_{t}+\left(M_{t}-M_{t-1}\right)+\left(A_{t}-A_{t-1}\right)+\left(B_{t}-B_{t-1}\right)
$$

oder in Wachstumsraten und als Einkommensquoten ausgedrückt

${ }^{116}$ Insbesondere sei hingewiesen auf die Darstellung der staatlichen Budgetbeschränkung im vorigen Kapitel (Abschnitt I) sowie die Diskussion über die Schuldfinanzierung der Zinszahlungen (Abschnitt II.2.f). 


$$
g+\operatorname{tr}+b(i-\phi)=t+\dot{m}+\dot{a}+\dot{b} .
$$

Zur Finanzierung seiner Ausgaben stehen einem Staat mit einem unabhängigen monetären System vier Einnahmequellen zur Verfügung: Die Besteuerung, die Geldschöpfung, die Veräußerung von öffentlichem Vermögen (m. a. W. Privatisierungserlöse) und eine Netto-Erhöhung der Staatsschuld $(t+\dot{m}+\dot{a}+\dot{b})$. Zudem ist es ihm auch möglich, die realen Ausgaben oder die Transferzahlungen zu senken $(\mathrm{g}+\mathrm{tr})$.

Im Vergleich dazu hat der Staat nur wenig Einfluß auf die Tilgungs- und Zinslast der vergangenen Kreditaufnahmen. Direkte Zahlungsverweigerung des Zinsdienstes (ib) ist für ein Industrieland mit offenen Kapitalmärkten wie das Vereinigte Königreich kaum möglich. Die Verweigerung von Zins- und Tilgungszahlungen im Inland wäre politisch unmöglich. Ein Verzug bei Zins- und Tilgungszahlungen der öffentlichen Auslandsverschuldung hätte einen Vertrauensverlust in den internationalen Wirtschaftsbeziehungen zur Folge. Neben direkten Vergeltungsmaßnahmen der ausländischen Kreditgeber, wie z. B. Sistierung von Handelskrediten, sind auch indirekte Schäden, wie z. B. begleitende Sanktionen der supranationalen Organisationen (z. B. IWF und Weltbank), höhere Risikopremien für zukünftige Kredite zu erwarten, welche zusätzliche Folgen auf die inländischen Wirtschaftsbedingungen hätten. ${ }^{117}$

Eine indirekte Tilgung läßt sich durch eine Erhöhung des nominalen Einkommens bewirken. Dabei stellt sich die Frage, wie ein derartiger Anstieg zu erreichen sei. Eine reale Einkommenserhöhung durch die Nachfragesteuerung ist auf Dauer unwahrscheinlich, wie die Ausführungen im ersten Kapitel, Abschnitt II zeigen. Wie weit die Regierung das reale Wachstum durch „supply-side“ Maßnahmen endogenisieren kann, ist eine zentrale und weitreichende Frage, deren Diskussion den Rahmen der Arbeit sprengen würde. ${ }^{118}$ Für die relativ kurze Frist dieser Betrachtung wird angenommen, da $B$ die Regierung einen ausreichenden Wachstumsschub zur Schuldensenkung auf dieser Weise nicht bewirken kann. Tilgung durch nominelles Wachstum läßt sich vor allem durch Inflation erreichen.

Die indirekte Tilgung durch Inflation sowie die fünf direkten Konsolidierungsmöglichkeiten durch Veränderungen der Einnahme- und Ausgabenpolitik sollen im

117 Siehe hierzu Sachs (1989), S. 26f. Wenn es neuerdings einige Beispiele von erfolgreicher Zahlungsverweigerung gibt, z. B. in verschiedenen südamerikanischen Ländern, hängt dies mit der Glaubwürdigkeit der Zahlungsunfähigkeit dieser Länder zusammen (hierzu Eichengreen und Lindert (Hrsg.) (1989)). Diese Bedingung war für das Vereinigte Königreich in den 80er Jahren nicht annähernd erfüllt.

${ }^{118}$ Hierzu z. B. Fels und von Furstenberg (1989). Judith Safford - 978-3-631-75199-2 
folgenden untersucht werden. Als Vorbedingung hierfür sollen jedoch zuerst die Einnahmeformen nach ökonomischen Kriterien voneinander unterschieden werden.

\section{2) Die Abgrenzung der Finanzierungsquellen}

\section{a) Kredit- versus Steuerfinanzierung}

Bei gegebenen Ausgaben und konstanten Geld- und Vermögensbeständen stehen sich im Rahmen einer sogenannten Differentialanalyse die Schuldfinanzierung und die Erhebung von Steuern gegenüber. Es gibt zwei wichtige Unterschiede zwischen diesen Finanzierungsformen. ${ }^{119}$ Der erste bezieht sich auf die Tauschhandlungen, die infolge Staatstätigkeit schlechthin stattfinden. Bei der Besteuerung findet lediglich ein Tausch zwischen Individuen als Steuerzahler und als Empfänger öffentlicher Ausgaben statt, der vom Staat koordiniert wird. Dieser tauschwirtschaftliche Aspekt der Steuerfinanzierung gilt im Sinne eines Ausgleichs zwischen erbrachten steuerlichen Leistungen und erhaltenen öffentlichen Leistungen selbstverständlich nur in der Aggregation, da Steuerzahler und Nutznießer öffentlicher Leistungen nicht identisch sein müssen. Da die Äquivalenz nicht für jedes einzelne Individuum gilt, haben Steuerzahlungen $Z$ wangscharakter.

Dagegen beruht die Kreditaufnahme auf einem Tausch, der aus freiwilligen Verhandlungen zwischen dem Staat als Schuldner und den privaten Haushalten als Gläubigern resultiert. Dies bedeutet für den Staat, daß er sich am marktwirtschaftlichen Proze $\beta$ beteiligen mu $\beta$ und er, wie die privatwirtschaftlichen Teilnehmer, dessen Bedingungen unterliegt. ${ }^{120}$

Der zweite Unterschied zwischen Besteuerung und Schuldenaufnahme betrifft das zeitliche Anfallen der Verbindlichkeiten zur Finanzierung der Staatstätigkeit. Bei der Steuerfinanzierung fallen die Verbindlichkeiten periodengleich mit den Ausgaben zusammen, weil die Steuereinnahmen unmittelbar die Ausgaben für öffentliche Leistungen finanzieren. Dagegen ermöglicht, wie im vorigen Abschnitt argumentiert wurde, die Schuldaufnahme eine intertemporale Lastverschiebung. Die freiwillige Bereitstellung der Einnahmen geschieht im Tausch gegen zukünftige Zinsund Tilgungszahlungen. ${ }^{121}$ Der Tausch zwischen Einnahmen und Ausgaben findet

119 Gandenberger (1981) S. 9ff., sowie Andel (1990), S. 359ff.

${ }^{120}$ Diese Erkenntnis, die einen wichtigen Beitrag zur „Last-Debatte“ der Verschuldung lieferte, geht auf Buchanan (1958) zurück. Zu einem Überblick dieser Debatte siehe Ferguson (1964).

${ }^{121}$ In diesem Sinne beschreibt Andel (1990) S. 359 die Schuldaufnahme als ein nichtdefinitives Finanzierungsinstrument, weil sie erst mit der Tilgung finanzpolitisch abgeschlossen sei 
nicht mehr innerhalb einer Periode, sondern über mehrere Perioden statt. Die Steuerzahler werden in der Zeit der Schuldenaufnahme entlastet, aber später bis zur kompletten Tilgung finanziell belastet. Nach dieser Interpretation bedeutet die Verschiebung der staatlichen Verbindlichkeiten also eine Zahlungsaufschiebung der öffentlichen Ausgaben.

\section{b) Kredit-versus Geldschöpfungsfinanzierung}

Der Unterschied zwischen Schuld- und Geldschöpfungsfinanzierung ist zunächst einmal nicht offensichtlich. Bei ihrer Beschaffung präsentieren sie sich beide buchungstechnisch als Verschuldungstransaktion zwischen der Finanzbehörde (als Schuldnerin) und der Notenbank (als Gläubigerin). ${ }^{122}$ Die Schuldtitel einer Kreditfinanzierung werden an Dritte weitergeleitet. Die Gläubiger sind dann die privaten Haushalte, welche Schuldtitel gekauft haben. Diese Gruppen stellen aber auch die Gläubiger von geldfinanzierten Krediten dar, weil sie das Notenbankgeld als Transaktionsmittel akzeptieren. Auch wenn die Unterbringung der Mittel unterschiedliche Formen hat, besteht auch hier eine Analogie zwischen Geld- und Kreditfinanzierung der Staatsausgaben.

In der Literatur werden häufig zwei Unterscheidungsmerkmale herangezogen, die als Ausgangspunkt der Überlegungen dienen können. ${ }^{123}$ Zum einen müssen im Gegensatz zur Verschuldung keine Zinszahlungen auf die Geldschöpfungsfinanzierung geleistet werden ${ }^{124}$ und zum anderen sieht die Geldschöpfung auch keinerlei Rückzahlungen vor. ${ }^{125}$ Es kann sogar die gegenteilige Wirkung eintreten: Löst sie einen Inflationsschub aus, dann kann der Staat seine Schulden dadurch tilgen.

\section{c) Kreditfinanzierung versus Verkauf von Aktiva}

Seit einigen Jahren ist die Veräußerung von öffentlichen Vermögen zu einer wichtigen Finanzierungsquelle für das öffentliche Budget im Vereinigten Königreich

122 In der Bundesrepublik findet diese Transaktion in solcher Form nicht statt, weil der Verkauf von staatlichen Schuldtiteln an die Zentralbank gesetzwidrig ist.

123 Siehe z. B. Schlesinger et al. (1993), S. 65.

${ }^{124}$ Solange der Grenzsteuersatz unter 100\% liegt, haben Bonds eine höhere Realverzinsung als Geld, dessen Nominalzins gleich Null ist. Damit - wie unten gezeigt wird - ist allerdings nicht sichergestellt, daß die Realverzinsung auf Bonds positiv ist.

${ }^{125}$ Ein drittes Unterscheidungsmerkmal stellt auf den Liquiditätsgrad der jeweiligen Finanzierungsformen, bzw. die Zahlungsmittelfunktion des Geldes ab. Diese Unterscheidung setzt den Einbezug des Zahlungsmittels in die Überlegungen voraus und ist in diesem Zusammenhang nicht zweckmäßig. 
geworden. Ihre Berücksichtigung in der staatlichen Budgetbeschränkung ist deshalb sinnvoll, jedoch wirft sie ein konzeptionelles Problem auf, das exemplarisch für das komplexe Unterfangen ist, eine ökonomisch sinnvolle Abgrenzung und Bewertung der Staatsverschuldung zu bestimmen. Zugang zur Problematik bekommt man durch die Vorstellung der staatlichen Vermögenslage in bilanzieller Form. Dabei stellt die herkömmliche Definition der Verschuldung nur einen Teil der staatlichen Verbindlichkeiten dar. Wenn Änderungen bei den anderen Bilanzkomponenten, z. B. den staatlichen Aktiva, sich auf die Verschuldung auswirken, dann sollten sie Berücksichtigung finden. ${ }^{126}$ Diese logische Erfordernis stößt jedoch auf enorme Schwierigkeiten bei der Erfassung und Bewertung. Die SNA ${ }^{127}$ Empfehlung beschränkt sich darauf, die Einnahmeströme aus der Veräußerung von öffentlichem Vermögen bei der Abgrenzung und Ermittlung der Neuverschuldung $\mathrm{zu}$ berücksichtigen.

Der Verkauf von öffentlichem Vermögen weist Ähnlichkeiten mit dem Verkauf von öffentlichen Schuldpapieren auf. Erstens resultieren die Änderungen der staatlichen Vermögensbestände aus einer freiwilligen Tauschhandlung zwischen öffentlichem und privatem Sektor. Zweitens ist aufgrund ihrer Auswirkungen auf die staatliche Vermögenslage ihr budgetwirksames Anfallen nicht auf die Periode der Veräußerung beschränkt. Drittens können staatliche Aktienemissionen verschiedene Portfolioumschichtungen auslösen. So wie staatliche Schuldemissionen auf Fremdkapitalmärkte können sie Verdrängungseffekte auf Eigenkapitalmärkten bewirken. ${ }^{128}$ Es läßt sich zeigen, daß Bond- und Aktivaverkäufe aus finanzpolitischer Sicht weitgehend äquivalent sind. ${ }^{129}$ Zur Vereinfachung der Darstellung wird zunächst von perfekter Voraussicht und vollkommenen Kapitalmärkten ausgegangen. Identische Produktionseffizienz im öffentlichen und privaten Sektor wird ebenfalls angenommen.

Unter diesen Bedingungen können die staatlichen Einnahmen aus der Veräußerung von öffentlichen Aktiva den Gegenwartswert ihrer versteuerten, zukünftigen Erträgen nicht übersteigen, da sonst der Staat keine privaten Käufer fände. Ist der Gegenwartswert des Unternehmens Null oder kleiner, so muß der Staat, um das Un-

${ }^{126}$ Für eine derartige staatliche Bilanzieren unter Berücksichtigung aller abdiskontierten realen Vermögensänderungen der Zukunft plädiert z. B. Buiter $(1983,1993)$. Schätzungen der britischen staatlichen Finanzen entsprechend einer solchen Bilanz machen Ashworth (1984) und Hills (1989). Bislang versucht lediglich Neuseeland seine öffentlichen Finanzen entsprechend einer umfassenden Bilanz zu verwalten, siehe IMF (1996) S. 48f.

127 Das „System of National Accounts“ (1993) SNA wird unter den supranationalen Instiutionen (UN, EU, OECD, IWF und Weltbank) regelmäßig vereinbart und gibt Empfehlungen zur Vorgehensweise bei der volkswirtschaftlichen Gesamtrechnung und anderen international relevanten Statistiken.

${ }^{128}$ Sinn und Sinn (1991), 93ff.

${ }^{129} \mathrm{Vgl}$. hierzu Mansoor (1993), S. 348ff. 
ternehmen veräußern zu können, entweder eine einmalige Subventionszahlung in Höhe der zukünftigen Verluste vorleisten (z. B. im Sinne eines negativen Preises) oder sich zu kontinuierlichen Subventionszahlungen verpflichten. Zur Zeit der Veräußerung hat der Staat einen einmaligen Mittelzufluß. In späteren Perioden dagegen sinken seine Einnahmen in Höhe der entgangenen Nettoeinkünfte. Ceteribus paribus steigen die zukünftigen Defizite.

Bei gegebener Geldfinanzierung können Aktivaverkäufe - analog zu Bondverkäufen - den staatlichen Haushaltsspielraum vorübergehend erweitern: Beide ermöglichen eine gegenwärtige Ausgabeerhöhung bzw. Steuersenkung. Privatisierungserlöse könnten aber auch zur Tilgung der Staatsschuld verwendet werden. Wenn der dem Verkaufserlös zugrunde liegende Diskontierungsfaktor den tatsächlichen finanziellen Opportunitätskosten entspricht, dann werden die zukünftigen Defiziterhöhungen exakt kompensiert. In beiden Fällen ist der Verkauf von Staatsschuldpapieren oder von Vermögensanteilen finanzpolitisch äquivalent. Die Aufhebung obiger Bedingungen modifiziert dieses Ergebnis. Die Auswirkungen einer Privatisierungspolitik unter weniger restriktiven Bedingungen wird im Abschnitt V unten wiederaufgenommen.

\title{
II. Strategie I: Der Inflationsmechanimus als Lösung des Schulden- problems
}

\begin{abstract}
„Inflation ..... is often a fiscal phenomenon: it is caused by governments with no alternative source of deficit finance resorting to money creation at a higher rate than the growth in money demand." 130
\end{abstract}

Der Weltbankbericht spricht mit diesem Zitat die altbekannte Interaktion zwischen Geld- und Fiskalpolitik an, die angesichts der geplanten Europäischen Währungsunion wieder viel Beachtung findet. ${ }^{131}$ Gemeint ist die Finanzierung der öffentlichen Haushalte durch Geldschöpfung. Dies hat vor allem negative Folgen, wenn die Steigerungsrate der Geldmenge den Anstieg der Geldnachfrage übersteigt. Eine solche exzessive Geldversorgung löst gemäß quantitätstheoretischer Zusammenhänge einen Inflationsprozeß aus. Möglicherweise kann sogar die Erwartung einer Geldmengenerhöhung eine Inflation auslösen. Nach der These von Sargent und Wallace (1981) erzeugt eine heutige Staatsverschuldung Erwartungen über höhere

${ }^{130}$ World Bank (1988) S. 57.

${ }^{131}$ Keynes (1923), S. 8f. hat die Inflation als Quelle der Budgetfinanzierung erkannt. Unter der Vielzahl der neueren Diskussionsbeiträge siehe z. B. Emerson et al. (1991), de Cecco und Giovannini (1989) und Gros und Thygesen (1992). 
Geldmengenwachstumsraten in der Zukunft. Da die Geldnachfrage von der erwarteten Inflationsrate abhängt, nimmt sie die Inflationszunahme vorweg und sinkt (die Umlaufgeschwindigkeit steigt). Damit steigt auch das heutige Preisniveau.

Werden noch die Folgen einer steigenden Inflationsrate für die nominelle Zins- und Einkommensentwicklung berücksichtigt, dann wird klar, daß geldpolitische Aspekte bei der Schuldenstabilisierung eine wichtige Rolle spielen.

Allerdings ist es ein schwieriges Unterfangen, die Auswirkungen und Kosten der Inflation zu erfassen. ${ }^{132}$ Hier werden lediglich die wichtigsten unmittelbaren Verteilungswirkungen auf das Einkommen und das Vermögen von Staat und Privaten beschrieben, wenn sich der Staat der Inflation bedient, um sich zusätzlichen Budgetspielraum zu verschaffen. Die indirekten und längerfristigen Auswirkungen auf das Wirtschaftsgeschehen, sowie die schwer erfaßbaren aber zweifelsohne signifikanten Folgen der erhöhten Unsicherheit bei wirtschaftlichen Entscheidungen bleiben somit unberücksichtigt.

Es gibt drei Gründe, weshalb eine Regierung Inflation zu Stabilisierungszwecken einsetzen könnte. Erstens verschafft sie dem Staat zusätzliche Einnahmen aus dem sogenannten Seignorage. Zweitens erhöht eine Inflation die nominalen Wachstumsund Zinseffekte auf die Schuldenquote. Drittens ergeben sich inflationsbedingte Einnahmen aus der Tatsache, daß der Staat Steuern erhebt und Ausgaben tätigt. Während der erste und dritte Effekt unabhängig von den Inflationserwartungen auftreten, beruht der zweite auf einer Täuschung der Staatsschuldgläubiger durch die unantizipierte Inflation.

\section{1) Schuldfinanzierung aus der Geldemission}

Wie im vorigen Abschnitt bereits erläutert, müssen im Gegensatz zur Verschuldung keine Zinszahlungen auf Geldemissionen geleistet werden, und zudem sind keine Rückzahlungen vorgesehen. Diese Attribute suggerieren eine für den Staat kostenlose Einnahmequelle. Der private Sektor ist für die Verfügbarkeit von Geld bereit, zinslose Kredite ohne Rückzahlung einzuräumen. Wenn der Staat bzw. die Notenbank allein das Recht zur Basisgeldemission besitzt, fällt ihm bei einer Erhöhung der Geldmenge eine Einnahme zu, die einen Finanzierungsbeitrag zum öffentlichen Haushalt leisten kann. Solche Einnahmen werden allgemein als „Seignorage" bezeichnet.

132 Einen umfassenden und anschaulichen Überblick über die Wirkungen der Inflation bieten Fischer und Modigliani (1978) und Garfinkel (1989). 
Grundsätzlich lassen sich zwei Definitionen von Seignorage in der Literatur unterscheiden. ${ }^{133}$ Die sogenannte "monetäre“ Seignorage ist eine Stromgröße, welche sich an der Kaufkraft des neu geschaffenen Basisgeldes orientiert. Gemessen wird sie an der zusätzlichen Geldhaltung der Öffentlichkeit, die ihre Realkasse den Preis- und Einkommensänderungen anzupassen versucht, und definiert ist als ${ }^{134}$

$$
\text { (1) } S_{m}=\frac{\dot{M}}{P}=\frac{M}{P} \cdot g_{m} \text {, }
$$

wobei $\mathrm{g}_{\mathrm{m}}$ die nominelle Wachstumsrate des Geldes $(\dot{\mathrm{M}} / \mathrm{M})$ und $\mathrm{M} / \mathrm{P}$ das reale Geldangebot im Sinne der Geldbasis ${ }^{135}$ sind.

Drückt man die Geldnachfrage nach quantitätstheoretischer Vorstellung als $M_{d}=k P Y$, oder, logarithmisiert, $\ln M_{d}=\ln k+\ln P+\ln Y$ aus, so ergibt sich für die Wachstumsrate (d. h. differenziert nach der Zeit):

$$
g_{m} \equiv \frac{d\left(\ln M_{d}\right)}{d t}=\frac{d(\ln k)}{d t}+\frac{d(\ln P)}{d t}+\frac{d(\ln Y)}{d t}=\pi+g_{y}
$$

Nimmt man ferner einen konstanten Kassenhaltungskoeffizient an $(\dot{\mathrm{k}}=0)$, so läßt sich die Wachstumsrate der nominellen Geldnachfrage aufteilen in eine Einkommens- und eine Inflationsausgleichskomponente. Letztere etabliert das gleichgewichtige Preisniveau, wo sich Geldnachfrage und Geldangebot entsprechen. Eingesetzt in der Seignoragegleichung ergibt sich

$$
\text { (2) } \quad S_{m}=\frac{M}{P} \cdot\left(\pi+g_{y}\right)
$$

Daraus wird ersichtlich, daß aus den Bestrebungen der Öffentlichkeit, ihre Realkasse bei positiver Inflationsrate konstant zu halten, der Staat Einnahmen in Höhe von $(\mathrm{M} / \mathrm{P}) \pi$ erzielt. Diese Komponente wird auch die „Inflationssteuer“ oder ,aktive“ Seignorage genannt ${ }^{136}$, wobei $\mathrm{M} / \mathrm{P}$ die Bemessungsgrundlage und $\pi$ den Steuersatz bezeichnet. Die Wachstumskomponente oder "passive“ Seignorage ergibt sich aus der stabilitätsgerechten Geldversorgung infolge einer gestiegenen Nachfrage nach Transaktionskasse in einer wachsenden Wirtschaft. Mit der unterstellten Einkommenselastizität der Geldnachfrage von eins, ist $\mathrm{g}_{\mathrm{y}}$ die Wachstumsrate des

133 Siehe z. B. Klein und Neumann (1990) S. 208f. sowie Gros und Thygesen (1992), S. 179f.

${ }^{134}$ Die Darstellung lehnt sich in vereinfachter Form an Friedman (1971), S. 849.

${ }^{135}$ Die Geldbasis oder „high-powered money“ ist definiert als das gesamte Zentralbankgeld der Kreditinstitute (Guthaben und Bargeldbestände) und der Bargeldumlauf.

${ }^{136}$ Die Bezeichnung geht auf Fischer (1982) zurück, S. 301 tu 
realen Sozialprodukts. ${ }^{137}$ Alle Größen lassen sich empirisch ermitteln, weshalb diese "cash flow" Bemessung der Seignorage in der Regel für empirische Zwecke herangezogen wird. ${ }^{138}$

Allerdings ist diese Definition unbefriedigend, weil die Nachfrage nach Realkasse instabil ist. Zunehmende Inflation erhöht die Opportunitätskosten der Geldhaltung, wodurch die Nachfrage nach Realkasse sinkt. ${ }^{139}$ Schwankungen der Geldnachfrage können auch konjunktur- und saisonbedingt oder infolge technischen Fortschritts im Zahlungsverkehr oder einer Ausdehnung der Schattenwirtschaft auftreten. Folglich können die Seignorage-Gewinne aus der Geldschöpfung variieren, auch wenn die Inflationsrate konstant bleibt.

Dem zweiten Ansatz zur Bemessung der Seignorage liegt ein Bestandskonzept zugrunde, das an die obige Unterscheidung zwischen Kredit- und Geldfinanzierung anknüpft. Das Bargeld und etwaige Pflichtreserven sind nach dieser Auffassung staatliche Verbindlichkeiten, auf die keine Zinszahlungen geleistet werden. Die Seignorage stellt danach die vom Publikum geleisteten „Opportunitätskosten“ der Geld- und der Pflichtreservehaltung dar, die der Staat abschöpft. Sie wird definiert als

$$
S_{0}=\frac{M}{P} \cdot i
$$

wobei für i ein geeigneter nominaler Zinssatz gewählt wird. ${ }^{140}$ Entsprechend der Fisher-Gleichung teilen sich die Seignorage-Einnahmen auf in die Inflationssteuer $\pi^{141}$ und den Realszins r:

$$
\text { (4) } \quad \mathrm{S}_{0}=\frac{\mathrm{M}}{\mathrm{P}} \cdot(\pi+\mathrm{r}) \text {. }
$$

Der „Opportunitätskosten“-Seignorage $S_{o}$ ist im Zeitverlauf stabiler als $S_{m}$, weil er nicht von der Geldnachfrage, sondern vom Geldbestand abhängt. Die Hauptschwierigkeit liegt in der empirischen Bestimmung des Seignorage $S_{0}$, da er die Wahl und

${ }^{137}$ Diese Annahme trifft Friedman (1971). Fischer (1982) nimmt in seiner empirischen Untersuchungen die Elastizitäten 1,0 und 0,5 an.

${ }^{138}$ Entsprechend Fischers (1982) Methodik wurde auf der Grundlage der IWF Statistik (International Financial Statistics „Reserve Money“ Zeile 14) die Seignorage beispielsweise in Dornbusch (1988), Drazen (1989) Giavazzi (1990) und Buiter und Kletzer (1991) berechnet.

${ }^{139}$ In der obigen Geldnachfragegleichung sinkt die Kassenhaltung k.

${ }^{140}$ Dies ist in der Regel die Umlaufrendite für festverzinsliche Wertpapiere.

141 Zur Vereinfachung wird vollständige Voraussicht der Wirtschaftssubjekte vorausgesetzt. Mit anderen Worten: die erwartete Inflationsrate $\left(\pi^{e}\right)$ der Fisher-Gleichung wird mit der tatsächlichen Inflationsrate gleichgesetzt. 
Ermittlung eines entsprechenden Zinssatzes i bzw. die Kenntnis des realen Zinssatzes erfordert.

Eine zweite Kritik, die auf beide Ansätze zutrifft, bezieht sich auf den implizit hergestellten Bezug zur Verwendungsseite der Notenbankbilanz (das Bargeld und die Sichtguthaben der Kreditinstitute) bei der Bemessungsgrundlage der Seignorage $(\mathrm{M} / \mathrm{P})$. Dadurch werden institutionelle und operationale Merkmale der Entstehung der monetären Basis vernachlässigt, welche die Höhe der Inflationssteuer für fiskalische Finanzierungszwecke entscheidend beschneiden. ${ }^{142}$ Aus der Entstehungsgleichung für die monetäre Basis wird deutlich, daß nicht jeder Geldschöpfungsvorgang einem kostenlosen Notenbankkredit an den Staat gleichzusetzen ist, so wie die beiden herkömmlichen Meßkonzepte der Seignorage dies suggerieren. Die Entstehungsquellen sind (unter Vernachlässigung kleinerer Restposten) die Geldschöpfung durch direkte Kreditvergabe an Private (Refinanzierungspolitik) oder an öffentliche Stellen und die indirekte Geldschöpfung beim Ankauf von Wertpapieren (Transaktionen der Offenmarktpolitik). Dazu kommt eine außenwirtschaftliche Komponente beim Erwerb von Devisenreserven. Erwirbt die Zentralbank vom Bankensystem oder vom Ausland verzinsliche Forderungsrechte, so führt sie Basisgeld in den monetären Kreislauf ein. Die Zinsdifferenz zwischen Zentralbankraten und einer marktgerechten Verzinsung bei in- und ausländischen Aktiva der Notenbank (Refinanzierungskredite und Devisen) kommt dem inländischen Privatsektor und dem Ausland und nicht dem Staat zu Gute. Dadurch entstehen der Zentralbank Kosten in Form von entgangenen Zinseinkünften. Zudem stellen die Verwaltungskosten des Zentralbanksystems, die Produktionskosten des Geldes und die Wertberichtigungen auf Auslandsforderungen zusätzliche Kosten dar. ${ }^{143}$ Alle diese Kosten werden aus dem gesamten Seignorage-Aufkommen finanziert und können demnach nicht zur Budgetfinanzierung verwendet werden.

Solche Divergenzen erklären die teilweise contra-intuitiven Ergebnisse, wenn die relative Bedeutung der Seignorage im internationalen Vergleich gemessen wird. ${ }^{144}$ Die Einkünfte der Regierung bilden nur eine Teilmenge der gesamten Kosten des privaten Sektors für die Geldhaltung, deren Größenordnung von der institutionellen und operationalen Gestaltung der monetären Basis abhängt. Deshalb ist es in die-

142 Z. B. die Beziehung zwischen den monetären und fiskalischen Behörden, die Höhe der Mindestreservevorschriften und die operationale Ausgestaltung der Geldpolitik. Siehe Spaventa (1989) S. 560 , Schlesinger et al. (1993), S. $60 \mathrm{f}$.

${ }^{143}$ Siehe Klein und Neumann (1990), 214ff. Gleichung 13, sowie Tabelle 2.

144 So waren beispielsweise bei Verwendung der IWF Statistik die Seignorage-Gewinne in der Bundesrepublik Deutschland zwischen 1960 und 1986 stets größer als in Großbritannien, obwohl die durchschnittliche Inflationsrate in Großbritannien in diesem Zeitraum deutlich höher war. Zu diesen Ergebnissen kommen Fischer (1982), Drazen (1989) und Gros und Thygesen (1992). Für eine Kritik der Meßkonzepte der Seignorage siehe Klein und Neumann (1990) und Gros und Thygesen (1992) S. 178f. 
sem Zusammenhang nicht sinnvoll, die herkömmlichen Definitionen der Seignorage zu verwenden, sondern es empfiehlt sich, diese Teilmenge anhand der direkten Geldfinanzierung zur Budgetbeschränkung, wie sie unter (3) definiert wurde, zu erfassen: ${ }^{145}$

$$
\text { (5) } \quad \dot{\mathrm{m}}=(\mathrm{g}-\mathrm{t})-\dot{\mathrm{a}}-\dot{\mathrm{b}}+\mathrm{b}(\mathrm{i}-\phi) \text {. }
$$

\section{2) Inflation zur Entwertung des Schuldenstands}

Die Budgetbeschränkung zeigt, wie die Entwicklung des Schuldenstands von der nominalen Wachstumsrate des Einkommens und des Zinses abhängt. Ein inflatorischer Schock erhöht das nominale Wachstum und führt zu einer realen Senkung der Schuldenquote. Die Schuldensenkung kommt einer Tilgung gleich, und die Staatsgläubiger erleiden einem entsprechenden Vermögensverlust. Der Bewertungseffekt tritt unabhängig von den inflatorischen Erwartungen auf und läßt sich analog zur ,aktiven“ Seignorage als eine Inflationssteuer auffassen, die auf die reale Schuldenbestandshaltung fällt.

Die inflatorischen Auswirkungen auf den Zinssatz hängen davon ab, wie die Finanzmärkte auf die Erosion des Schuldenstandes reagieren. Wenn die zukünftige Preisentwicklung vollkommen antizipiert wird, sind die Wertpapierinhaber frei von Geldillusion und werden ceteribus paribus bestrebt sein, den realen Wert ihrer Aktiva konstant zu halten und folglich ihre realen Wertpapierbestände wieder aufzubauen. Sie fordern einen höheren Nominalzins zur Entschädigung ihrer Vermögensverluste. Entsprechend der Fisher-Hypothese wird bei vollständiger Voraussicht der Realzins von Preisänderungen nicht tangiert. ${ }^{146}$ Es gilt die Fisher Gleichung (wieder unter der Annahme vollständiger Voraussicht, d. h. $\pi^{\mathrm{e}}=\pi$ ).

(6) $\quad \mathrm{i}=\overline{\mathrm{r}}+\pi$,

bei der sich der nominale Zinssatz der Inflation anpaßt. Der nominale Zinsanstieg erhöht den Finanzierungsbedarf für den Staat. Der inflationsbedingte Tilgungseffekt infolge des nominalen Einkommensanstiegs wird genau durch die dafür notwendige Neuverschuldung ausgeglichen. Die Inflationssteuer auf den Schuldenbestand wird über den nominalen Zinsanstieg zum Inflationsausgleich auf den Staat zurückgewälzt.

\footnotetext{
145 Klein und Neumann (1990) S. 210.

146 Von anderen Einflüssen auf den Realzins, wie z. B. variablen Risikoprämien oder Transaktionskosten, wird damit abstrahiert.
} 
Anders verhält es sich im Falle einer unerwarteten Inflation. Der hergestellte Nexus zwischen nominalem Zinssatz und Inflation hebt sich auf, da die Wertpapierinhaber keinen Inflationsausgleich beanspruchen. Bei konstantem Nominalzins muß sich der Realzins anpassen, um die Erfüllung von (6) zu gewährleisten. Deswegen steigt der nominale staatliche Finanzierungsbedarf infolge der Inflation nicht an und die reale Neuverschuldung bleibt gleich. Die inflationsbedingte reale Wertminderung des Schuldenbestands tritt unverändert auf, was de facto eine reale Schuldentilgung bedeutet. In diesem Falle wird die Vermögensabwertung der Staatsschuldgläubiger nicht durch einen Zinsanstieg kompensiert. Der Staat erhält implizite Einnahmen in Form einer Inflationssteuer mit der Rate $\pi$ auf den Schuldenbestand [b in Gleichung (5)]. Damit wird deutlich, daß die Reaktion des Nominalzinses auf die Staatsschuld im Gefolge der Inflation entscheidend ist für den Umverteilungseffekt zwischen Schuldner und Gläubiger.

Das Fisher-Theorem ließe erwarten, daß eine nominale Zinsanpassung Eingang in die Kreditverträge findet. Die Vermögensumschichtung zugunsten der öffentlichen Hand, die über die Inflationssteuer auf die Schuldenbestandshaltung anfällt, hängt vom Ausmaß der Geldillusion der Staatsgläubiger und der durchschnittlichen Restlaufzeit der bestehenden Schuldenvereinbarungen ab: diese Faktoren bestimmen die Geschwindigkeit der Inflationsanpassung. Infolge der in der Nachkriegszeit gestiegenen Kapitalmarkteffizienz (z. B. sinkende Informationskosten) und der von den Gläubigern geforderten Verkürzung der Fristenstruktur der öffentlichen Schuld in allen Industrieländern ist der Spielraum des Staates, die Staatsverschuldung durch Inflation zu stabilisieren - wie dies in Deutschland und Frankreich in den 20 er Jahren geschah - erheblich eingeschränkt worden. ${ }^{147}$ Die Inflationssteuer auf dem Schuldenstand wäre also eine ineffiziente und unbedeutende staatliche Einnahmequelle geworden.

Allerdings widersprechen diesen theoretischen Ergebnissen empirische Evidenzen zumindest teilweise, weil kurzfristig eine negative Korrelation zwischen Inflation und Realzins zu erkennen ist. D. h. die Inflation wird zumindest kurzfristig nicht richtig antizipiert, und eine Nominalzinsanpassung entsprechend dem FisherTheorem findet erst mit einer zeitlichen Verzögerung statt. ${ }^{148}$ Demzufolge kann der Staat zumindest in dieser Anpassungszeit seine Schuldenlast durch Inflation min-

${ }^{147}$ Blanchard et al. (1986b) S. 133ff. Indexierte Schuldverschreibungen verhindern auch die Ausbeutung der Inflationssteuer, Schlesinger, (1993) S. 51. Im Vereinigten Königreich werden indexierte Staatsschuldpapiere ausgegeben. Darauf wird im vierten Kapitel, Abschnitt II.3 eingegangen.

${ }^{148}$ Siehe z. B. Summers (1983) und Viren (1986). 
dern. Damit gewinnen auch empirische Schätzungen der staatlichen Schuldentilgung aufgrund der Inflation an Plausibilität. ${ }^{149}$

\section{3) Inflationswirkungen auf das Steuer- und Ausgabensystem}

Inflationäre Entwicklungen wirken auch auf die Struktur des bestehenden Steuersystems und verstärken darin enthaltene Verzerrungen. ${ }^{150}$ Es handelt sich dabei um Auswirkungen, die auch bei antizipierten Preiserhöhungen auftreten. Bekannt in diesem Zusammenhang ist die Wirkung der „kalten Progression“ bei der Einkommensteuer. Die „kalte Progression“ beschreibt die erhöhte Steuerbelastung, wenn das nominale Einkommen infolge einer Inflation in eine höhere Progressionsstufe verlagert wird, obwohl kein Anstieg des Realeinkommens stattgefunden hat. Die staatlichen Einnahmen aus der Körperschaftssteuer erhöhen sich ebenfalls infolge der Inflation: Erstens sinkt der gegenwärtige abdiskontierte Wert von Abschreibungen bei steigender Inflation, wenn diese nur zu Anschaffungskosten zugelassen werden. Zweitens verursacht ein Anstieg der Verkaufspreise gegenüber den Einkaufspreisen bzw. Herstellungskosten einen steuerpflichtigen nominalen Gewinnanstieg, der gemäß der „kalten Progression“ steuerlich belastet wird. ${ }^{151}$ Im Vereinigten Königreich ist die Regierung seit 1977 gesetzlich verpflichtet, den Steuertarif der Inflation im jährlichen Haushaltsplan anzupassen. ${ }^{152}$ Die persönlichen Freibeträge der Einkommensteuer sind sogar in den 80 er Jahre real um über $25 \%$ erhöht worden. Die Wertzuwachssteuer wurde 1982 ebenfalls indexiert, so daß die Scheingewinne aus nominalem Kapitalzuwachs nicht mehr besteuert werden. ${ }^{153}$

Wichtiger allerdings scheinen die Auswirkungen der Besteuerung von nominalen Zinseinkünften zu sein, ${ }^{154}$ da eine Inflation das steuerpflichtige Zinseinkommen aufbläht, wenn es nach dem Nominalwertprinzip besteuert wird. Nach der FisherGleichung (6) (bei vollständiger Voraussicht) besteht der Nominalzins aus zwei Komponenten. Die Realzinskomponente $r$ ist eine Entlohnung für gegenwärtigen Konsumverzicht und stellt somit Einkommen dar. Die Inflationskomponente $\pi$ ist eine Prämie, die lediglich den Kaufkraftverlust durch die Inflation ausgleichen soll.

${ }^{149}$ Fischer und Modigliani (1978) finden signifikante Umverteilungseffekte zugunsten der verschuldeten öffentlichen Haushalte und folglich zu Lasten der Staatsgläubiger, S. 823ff. Siehe auch die empirischen Schätzungen von Cassel, D. (1982) und vor allem Cukierman et al. (1985).

${ }^{150}$ Einen kurzen Überblick hierzu gibt Issing (1993), S. 213ff. Siehe auch die dort zitierte Literatur.

151 Fischer und Modigliani (1978) S. 819.

152 So bestimmt es das Rooker-Wise Amendment zum Finanzgesetz (1977), wobei Ausnahmefälle zugelassen werden. Britton (1991), S. 317.

153 Byatt (1989), S. 357.

${ }^{154}$ Siehe z. B. Schlesinger (1993), S. 50f und Fischer ynd Modigliani (1978), S. 8.17 $75199-2$ 
Beim Nominalwertprinzip werden jedoch beide Komponenten als Einkommen behandelt und besteuert. Selbst bei vollständig antizipierter Inflation muß der Nominalzins überproportional ansteigen, um die steuerliche Belastung auf der Inflationskomponente zu kompensieren. Die Fisher-Gleichung (6) nach Nominalwertbesteuerung ist

$$
i(1-\tau)=\bar{r}+\pi \text { bzw. } i=\frac{\bar{r}+\pi}{(1-\tau)}
$$

Daraus ist ersichtlich, daß bei einem konstanten Grenzsteuersatz $(0<\tau<1)$ der Nominalzins um mehr als die korrekt antizipierte Inflationsrate $\pi$ steigen muß, um den realen Ertrag konstant zu halten. ${ }^{155}$ Geschieht dies nicht, erfolgt ein Einkommenstransfer vom Steuerzahler zum Staat.

Die Auswirkungen von Inflation auf die Staatsausgaben hängen im wesentlichen davon ab, ob die Verteuerungsraten der einzelnen Ausgaben sich von der gesamtwirtschaftlichen Inflationsrate unterscheiden, bzw. ob und wie die Ausgaben letzterer angepaßt werden. ${ }^{156}$ Der Deflator für Investitionsausgaben liegt häufig höher als der gesamtwirtschaftliche, weil Baulichkeiten und andere Konstruktionen sich bei Inflation überdurchschnittlich verteuern. Somit steigen die Kapitalausgaben bei Inflation überproportional an. Die Ausgaben, beispielsweise für das Erziehungsund Gesundheitswesen, die Justiz und die Polizei sind überwiegend arbeitsintensiv. Deswegen hat die Lohnentwicklung, u. a. die Inflationsanpassung der Löhne, einen besonderen Einfluß auf das Wachstum dieser Ausgaben. Auf diesen Zusammenhang wird unter Abschnitt IV.3.a noch näher eingegangen.

\section{Strategie II: Steuererhöhungen unter Berücksichtigung der Auf- kommenselastizität}

Als weitere Konsolidierungsoption kann der Staat versuchen, seine Verschuldung durch eine Erhöhung der Steuern und Sozialabgaben zu stabilisieren bzw. zu senken. ${ }^{157}$ Das Aufkommen aus diesen zwei Quellen hängt von der Flexibilität ihrer Ausgestaltung im Hinblick auf juristische, politische und ökonomische Beschrän-

155 Gebauer (1982), S. 79.

156 Vgl. hierzu Tanzi (1985), S. 160ff.

${ }^{157}$ Der Staat erzielt auch Einnahmen aus Gebühren und sonstigen Abgaben. Wegen ihrer geringen relativen Bedeutung werden diese Finanzierungsquellen night; weiter beachtet $31-75199-2$ 
kungen ab. ${ }^{158}$ Daraus ergibt sich der Spielraum der politischen Entscheidungsträger, die Steuereinnahmen zu variieren. Da die Schuldenkonsolidierung Steuererhöhungen erfordert, sind die oberen Flexibilitätsgrenzen von besonderem Interesse.

Das potentielle Aufkommen einer gegebenen Steuer ergibt sich zusammen aus dem durchschnittlichen Steuersatz multipliziert mit der Bemessungsgrundlage. Zwischen zwei Steuern mit identischen Bemessungsgrundlagen und Steuersätzen kann das erzielte Aufkommen jedoch stark variieren, je nachdem wie effizient die Steuererhebung erfolgt. Das budgetwirksame Steueraufkommen hängt deshalb von drei Politikvariablen ab: Vom Steuersatz, von der Bemessungsgrundlage und im weitesten Sinne von der Effizienz des Steuerstaats.

Die oberen Elastizitätsgrenzen des Steueraufkommens werden zunächst in unmittelbarer Abhängigkeit von diesen Größen untersucht. Als Grundlage zu den Überlegungen dienen die Optimalsteuer-Theorie sowie die „Public Choice“ Theorie. Es zeigt sich, daß eine Erhöhung des Steuersatzes sich negativ auf die Bemessungsgrundlage und die Effizienz des Steuerstaats auswirkt. Daraus lassen sich endogene Grenzen der Flexibilität bzw. der Aufkommenselastizität der Besteuerung ableiten. Die Verwirklichung des Europäischen Binnenmarktes wird die Flexibilität der Steuereinnahmen ebenfalls beeinflussen. Zum einen hat die Kommission konkrete $\mathrm{Maßnahme} \mathrm{zur} \mathrm{Steuerharmonisierung} \mathrm{erlassen.} \mathrm{Zum} \mathrm{anderen} \mathrm{erfolgt} \mathrm{eine} \mathrm{„sponta-}$ ne" Steuerharmonisierung aus der wachsenden Integration und der erhöhten Mobilität von Produktionsfaktoren, Konsumenten und Unternehmen. Schließlich können kollektive Bewegungen des Steuerwiderstandes politische Grenzen der Besteuerung setzen. Alle drei Phänomene können die Elastizität der Steuereinnahmen begrenzen.

\section{1) Die Analyse der Laffer-Kurve}

Der Glaube, daß eine Aufkommensgrenze der Besteuerung existiert, hat eine längere Tradition in der Finanzwissenschaft. Bereits 1776 schreibt Adam Smith in „The Wealth of Nations":

„High taxes, sometimes by diminuishing the consumption of the taxed commodities, and sometimes by encouraging smuggling, frequently

158 Juristische Beschränkungen werden im folgenden vernachlässigt. Da in Großbritannien keine konstitutionellen oder verfassungsmäßigen Einnahmengesetze existieren, hat die Regierung juristisch eine praktisch unbegrenzte Freiheit bei der Einnahmegestaltung. 
afford a smaller revenue to government than what might be drawn from more moderate taxes.“ 159

Nach Smith sind dem maximalen Aufkommen einer Steuer endogene Grenzen gesetzt, weil einer ansteigenden Steuerbelastung mit zunehmenden Substitutions- und Steuer-Ausweichreaktionen begegnet wird, die eine Steuersatzerhöhung überkompensieren können.

Diese Überlegungen fanden Ende der 70er Jahre durch die Arbeiten von Laffer und anderer "Supply side“ Ökonomen erneute Aufmerksamkeit. ${ }^{160}$ Die sog. LafferKurve zeigt analytisch, daß eine obere Aufkommensgrenze der Besteuerung existieren muß. Sie ist in Abbildung 6 dargestellt. Bei einem Steuersatz von Null ist das Steueraufkommen Null. Der Spitzensatz einer Steuer mit breiter Bemessungsgrundlage liegt bei $100 \%$. Dann ist auch das Aufkommen gleich Null. ${ }^{161}$ Gilt für die Aufkommensfunktion $T^{\prime}(0)>0,{ }^{162}$ dann muß ein Steuersatz $\tau^{*}$ existieren, bei $\operatorname{dem} T^{\prime}(\tau)=0$ und das maximale Aufkommen $T^{*}$ erreicht wird. Somit stellt $\tau^{*}$ eine Grenze der Besteuerung dar.

\section{Abbildung 6: Die Laffer-Kurve}

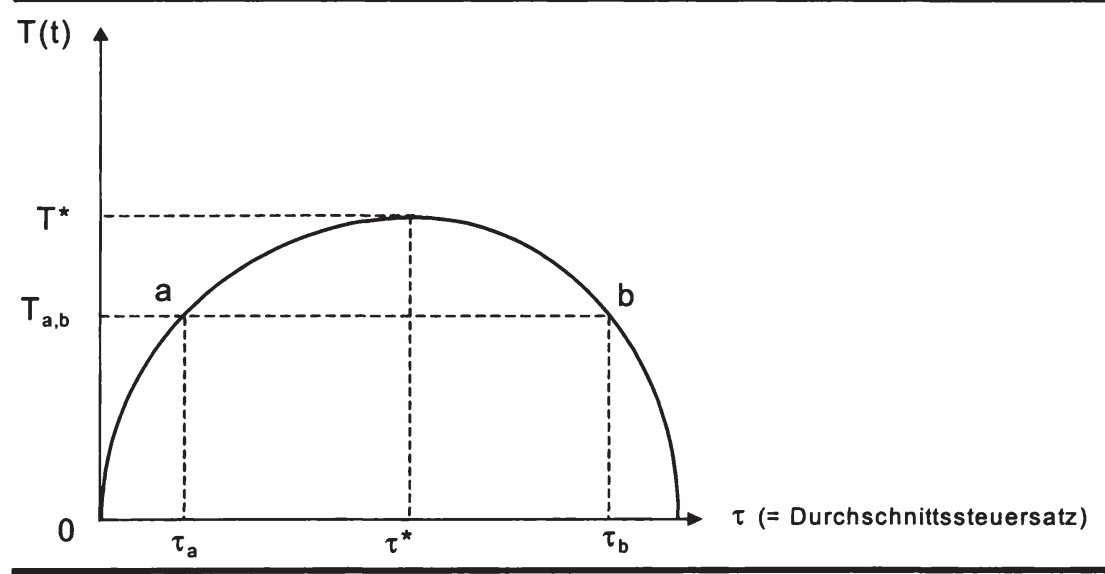

${ }^{159}$ Smith, A. (1776), S. 884.

160 Für eine Darstellung und ausführliche Diskussion der Laffer-Kurve siehe den Sammelband von Meyer (Hrsg.) (1981).

${ }^{161}$ Einzelne indirekte Steuern auf Einfuhren, Luxusgütern, oder „bads“, wie beispielsweise Zigaretten und Alkohol, können über $100 \%$ des Produzentenpreises erreichen, so daß sich das Aufkommen asymptotisch Null annähert. Diese Tatsache ist aber unwichtig für die Grundaussage der Laffer-Kurve und wird nicht weiter beachtet.

162 Die Stetigkeit und Differenzierbarkeit der Aufkommensfunktion werden vorausgesetzt. 
Der Verlauf der Kurve, wonach das zusätzliche Steueraufkommen mit steigendem Steuersatz marginal abnimmt, erklärt sich nach dem Laffer'schen Ansatz aus Substitutionshandlungen der Steuerzahler bei steigendem Grenz- und damit steigendem Durschschnittssteuersatz. Dieser Zusammenhang läßt sich durch ein einfaches Einkommenskonzept verdeutlichen. Das gesamte Einkommen (Y) wird aus dem Faktor Arbeit (L) hervorgebracht, welcher zu einem durchschnittlichen Lohn (vor Steuern) $\mathrm{w}$ angeboten wird: $\mathrm{Y}=\mathrm{wL} .{ }^{163}$ Das gesamte Einkommen wird zu einem konstanten Grenzsteuersatz $\tau$ besteuert (Proportionalsteuer: Grenz- = Durchschnittssteuersatz). Die Aufkommensfunktion ist dann

$$
\text { (1) } \quad T=\tau(w L)
$$

Da der Steuersatz sowohl den Lohn (w) als auch die angebotene Menge (L) beeinflußt, hat eine Erhöhung des Steuersatzes $\tau$ drei Auswirkungen auf das Aufkommen:

$$
\frac{d T}{d \tau}=w L\left[1+\frac{\tau}{L} \frac{d L}{d \tau}+\frac{\tau}{w} \frac{d w}{d \tau}\right] .
$$

Die erste Komponente ist der reine Budgeteffekt. Sie ergibt das Steueraufkommen, wenn keine Verhaltensreaktionen auf die Steuererhöhung auftreten. Ein Aufkommensanstieg erfolgt proportional zum Steuertarif, wenn die Bemessungsgrundlage in keiner Beziehung zum Steuersatz steht. Die zweite Komponente ist die Wirkung der Steuer auf das Arbeitsangebot: Durch die Steuererhöhung sinkt der Nettolohn und löst einen Substitutions- und einen Einkommenseffekt aus. Da Freizeit relativ „preiswerter“ geworden ist, wird Freizeit für Arbeit substituiert. Auf der anderen Seite muß zur Aufrechterhaltung des gegebenen Einkommens mehr gearbeitet werden. Es wird angenommen, da $B$ der Substitutionseffekt dominiert. Folglich ist der Gesamteffekt negativ $(\mathrm{dL} / \mathrm{d} \tau<0)$. Die dritte Komponente erfaßt die Wirkung einer Lohnänderung infolge der Steuererhöhung. Die Arbeitnehmer versuchen, den Rückgang des Nettolohns durch eine Erhöhung des Bruttolohns (höhere Lohnforderungen) zu kompensieren $(d w / d \tau>0)$.

Die Argumentation der Laffer-Kurve und der daraus abgeleitete Steuersatz $\tau^{*}$ fuBen auf Effizienzüberlegungen der Optimalsteuer-Theorie. ${ }^{164}$ Die Form der Kurve ergibt sich aus den drei verschiedenen Reaktionen auf den Steuersatz, wobei beim

${ }^{163}$ Vgl. für eine ähnliche Vorgehensweise vgl. Fullerton (1982) und Peacock (1989). Cantor, Joines und Laffer (1981) verwenden ein zwei-Faktoren-Modell. Ihre Argumentation ist jedoch ähnlich.

164 In der Abbildung 6 wäre bei jedem Steuersatz $\tau_{b}>\tau^{*}$ das gleiche Steueraufkommen mit einem niedrigeren Steuersatz $\tau_{a}<\tau^{*}$ zu erreichen. Der höhere Steuersatz $\tau_{b}$ verursacht höhere Effizienzverluste und ist deswegen ineffizient. In diesem Zusammenhang sei daran erinnert, daß die $\mathrm{Zu}-$ satzlast einer gegebenen Steuer mit dem Grenzsteuersatz im Quadrate wächst, Cullis und Jones (1992), S. 185. 
Arbeitsangebot der Substitutionseffekt den Einkommenseffekt immer überwiegt. Dies bedeutet, daß die Arbeitnehmer sich entscheiden, bei steigenden Steuersätzen weniger $\mathrm{zu}$ arbeiten.

Unberücksichtigt bei der Laffer'schen Analyse bleiben beispielsweise die bereits vom Smith (1776) erkannten Steuerausweichungsreaktionen bei steigender Steuerlast. Diese können zwei Formen annehmen. Steuerhinterziehung als Erstes - die vorsätzliche Unterdeklaration von steuerpflichtigen Einkünften - umfaßt die Betätigung in der sogenannten Schattenwirtschaft und die ungesetzmäßige Steuerflucht. ${ }^{165}$ Sie scheint in den Industrieländern weit verbreitet $\mathrm{zu}$ sein, und zumindest bis in die $80 \mathrm{er}$ Jahre stieg sie an. ${ }^{166}$ Steuervermeidung als Zweites ist die legale Ausnutzung von Steuerlücken (absetzbare Kosten, steuerfreie Einkünfte, etc.), Zahlungsaufschub und Arbitragemöglichkeiten zwischen Individuen oder Einkommensklassen, um die deklarationspflichtigen Einkünfte zu mindern. ${ }^{167}$ Die Tendenz zu Steuervermeidung scheint auch in den letzten Jahren zugenommen zu haben. ${ }^{168}$

Steuerhinterziehung und Steuervermeidung sind in vieler Hinsicht Substitute, aber auch möglicherweise Komplemente. ${ }^{169}$ Deswegen werden sie in der Literatur oft in den Begriffen „Steueraversion“ oder „Steuerwiderstand“ zusammengefaßt. ${ }^{170}$ Das Ausmaß der Steueraversion gibt Auskunft über die subjektive Beurteilung des Steuersystems durch die Steuerzahler. Wird das Steuersystem als schlecht beurteilt, steigt die Steueraversion und die Effizienz des Steuersystems - im Sinne des maximalen Aufkommens bei gegebener Bemessungsgrundlage - wird sinken. Die wichtigsten Gründe für Steueraversion scheinen erstens der Glaube zu sein, daß die absolute Last der Besteuerung zu hoch liegt und zweitens die Wahrnehmung einer ungerechten relativen Belastung durch die Progression des Systems. ${ }^{171}$ Daraus läßt

165 Im obigen Modell äußert sich dies in einem Absinken von L, welches nur die „offizielle“ bzw. "legale“ Arbeitszeit erfaßt.

166 Schätzungen der Schattenwirtschaft in Europa und Nordamerika geben einen Anteil der wirtschaftlichen Aktivität von circa 10\%, die sich einer steuerlichen Erfassung entzieht. Siehe der kurze Überblick bei Blankart (1994), S. 230ff sowie Pyle (1989) Kap. 2-4.

${ }^{167}$ Stiglitz (1986), S. 3.

${ }^{168}$ Siehe Pyle (1993), S. 60f. und die dort zitierte Literatur.

169 Bei der Entscheidung für Steuervermeidung und -hinterziehung müssen sich die jeweiligen Grenzerträge ausgleichen. Eine Erhöhung des Grenzertrags der Steuerhinterziehung senkt den relativen Ertrag der Steuervermeidung. Engagiert zwecks Steuervermeidung gibt ein Steuerberater möglicherweise Informationen (bewußt oder unbewußt) über Möglichkeiten der Steuerhinterziehung weiter. Cross und Shaw (1982), S. 36.

${ }^{170}$ Siehe z. B. Cross und Shaw (1982) und Waud (1986, 1988).

171 Weniger wichtig, aber auch zu erwähnen ist die Kritik an der Effizienz oder den Kosten der öffentlichen Verwaltung, die als X-Ineffizienz oft in der „Public Choice“ Literatur betont wird, vgl.

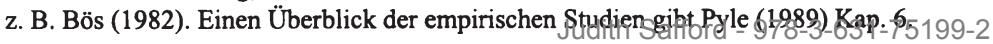


sich schließen, daß die Bereitschaft Steuern zu zahlen, in erster Linie vom durchschnittlichen bzw. marginalen Steuersatz abhängt.

Der Steuerstaat steht selbstverständlich nicht ohne Mittel gegen eine steigende Steueraversion dar. Er kann und wird versuchen, Gegenmaßnahmen zu ergreifen (etwa durch erhöhte Kontrollen und Strafen sowie die Schließung von „Steuerlükken") und somit seine Einnahmeverluste senken. Solche Maßnahmen haben jedoch mit steigenden Steuersätzen sinkende Grenzerträge. ${ }^{172}$ Die Erkenntnis, da $\beta$ sehr hohe Steuersätze unergiebig sind, weil sie trotz Kontrollen und Strafen weitgehend vermieden oder hinterzogen werden, ist ein Grund für Steuerreformen in beispielsweise den USA, dem Vereinigten Königreich oder Australien gewesen.

Verschiedene Autoren haben versucht, diese Überlegungen in der Analyse der Laffer-Kurve einzuarbeiten. ${ }^{173}$ Eine „Public Choice“ Erweiterung des Laffer-Ansatzes zeigt Parallelen zu den neueren Ansätzen der Optimalsteuer-Theorie, welche die Verteilungsgerechtigkeit und die administrativen Kosten des Steuersystems zu berücksichtigen versuchen. ${ }^{174}$ Neben der allokativen Effizienz erwägt die erweiterte Laffer-Kurve die subjektive - d. h. vor allem die verteilungspolitische - Akzeptanz des Steuersystems. In einem Steuersystem, das subjektiv als zu belastend oder ungerecht empfunden wird, steigt die Steueraversion. Die Reaktion der Steuerbehörde auf zunehmende Steueraversion läßt sich auch berücksichtigen: Zusätzliche Aufwendungen (Steuerreform, größere Überwachungs- und Kontrolltechniken, u. ä.) zur Durchsetzung des Systems erhöhen ihre Effizienz und somit das Steueraufkommen bei gegebenem Steuersatz.

Unter der Annahme, daß aufgrund von Verlusten durch Steueraversion de facto nur ein Anteil $[a(\tau)]$ des Volkseinkommens tatsächlich deklariert wird, läßt sich die Steueraufkommensfunktion formulieren als

$$
\text { (2) } \quad T=\tau[a(\tau)] Y \text {. }
$$

Eine Erhöhung des Steuersatzes ergibt

$$
\frac{\mathrm{dT}}{\mathrm{d} \tau}=\mathrm{aY}\left[1+\frac{\tau}{\mathrm{a}} \frac{\partial \mathrm{a}}{\partial \tau}+\frac{\tau}{\mathrm{Y}} \frac{\mathrm{dY}}{\mathrm{d} \tau}\right] .
$$

Die erste Komponente ist wieder der direkte Budgeteffekt bezogen auf das deklarierte Einkommen. Die dritte Komponente umfaßt die Anreizeffekte, welche die

\footnotetext{
172 Das optimale Ausmaß von Durchsetzungsmaßnahmen wird modelltheoretisch als herkömmliches Optimierungsproblem bei Unsicherheit behandelt. Siehe z. B. Pyle (1993), S. 89f.

${ }^{173}$ Siehe z. B. Waud (1986, 1988), Peacock (1989), Pyle (1989) Kap. 5.

${ }^{174}$ Siehe z. B. Slemrod (1990). 
herkömmliche Laffer-Kurve beschreibt $(\mathrm{dY} / \mathrm{d} \tau<0)$. Neu verglichen zu $(1)$ ist der Aversionseffekt, der entsprechend der obigen Argumentation negativ ausfällt $(\partial \mathrm{a} / \partial \tau<0)$. Ohne Spezifikation der Funktion $\mathrm{a}(\tau)$ ist es nicht möglich, $\tau_{\mathrm{a}}{ }^{*} \mathrm{zu}$ ermitteln. Es ist jedoch klar, daß $\tau_{\mathrm{a}}{ }^{*}<\tau *$ und das Aufkommen $\mathrm{T}_{\mathrm{a}}{ }^{*}<\mathrm{T}^{*}$ ist. Die Abbildung 7 zeigt die zwei Kurven im Vergleich.

\section{Abbildung 7: Die Laffer-Kurve und die Effizienz des Steuerstaates}

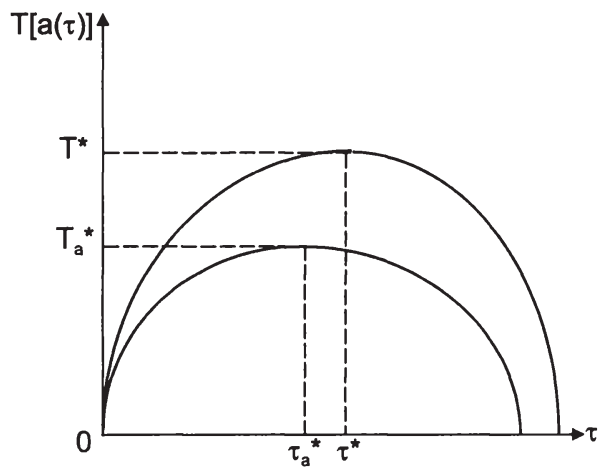

Ziel der Analyse war es, die Elastizität der Steuereinnahmen zu untersuchen, um den Spielraum für eine Aufkommenserhöhung festzustellen. Die erweiterte LafferKurve zeigt, da $\beta$ eine endogene Grenze der Besteuerung existiert, oberhalb derer das Steueraufkommen sich nicht erhöhen läßt. Die Fragen, wie die Kurven für die wichtigsten Steuern in der Realität aussehen und ob Aufkommenserhöhungen möglich sind, ist jedoch schwierig zu beantworten. Eine Analyse der kritischen Werte für die relevanten Elastizitäten der einfachen Laffer-Kurve zeigt, daß wenn ein höherer Steuersatz zu einer Bruttolohnerhöhung führt ( $d w / d t>0)$, dann muß das Arbeitsangebot sehr elastisch reagieren $(\tau / \mathrm{L} * \mathrm{dL} / \mathrm{d} \tau<-1)$, wenn das Steuersystem sich auf dem absteigenden Ast der Laffer-Kurve befinden soll. Nach den meisten Untersuchungen treten hierfür ausreichende Substitutionseffekte erst bei sehr hohen $\tau$ Werten auf. ${ }^{175}$ Waud (1986) überprüft eine Aufkommensfunktion, welche die Steueraversion (modelliert als kubische Kostenfunktion) berücksichtigt und ermittelt eine theoretische Aufkommensgrenze der Besteuerung bereits bei $\tau_{\mathrm{a}}{ }^{*}=0,33 .{ }^{176}$

175 Für einen Wert von $t^{*}=0,5$ müssen bei der einfachen Laffer-Kurve die Elastizitäten sehr hoch sein: so ist beispielsweise für $\eta_{\mathrm{s}}=1 \eta_{\mathrm{d}}=-\infty$, für $\eta_{\mathrm{s}}=2 \eta_{\mathrm{d}}=-4$ und für $\eta_{\mathrm{s}}=3 \eta_{\mathrm{d}}=-3$. Für alle Werte von $\eta_{s}<1$ liegt der Steuersatz bei unrealistisch hohen Werten; selbst bei $\eta_{d}=-\infty$ müßte der Steuersatz bei $67 \%$ liegen. Siehe hierzu auch Blinder (1981) S. 87, Fullerton (1982) S. 15 und Peacock (1989) S. 33.

${ }^{176}$ Waud (1986), S. 223. 
Dieses Ergebnis findet aber weder in der Erfahrung in den U.S.A. ${ }^{177}$ noch in empirischen Arbeiten über das Vereinigte Königreich Unterstützung. Ökonometrische Untersuchungen finden signifikante Anreizeffekte der Steuersatzsenkungen der 80er Jahre lediglich bei verheirateten Frauen. Steuersenkungen für diese Bevölkerungsgruppe erbringen jedoch keine wesentliche Zusatzeinnahmen. ${ }^{178}$

\section{2) Kollektive Bewegungen des Steuerwiderstands}

Nicht berücksichtigt bei der erweiterteten Laffer-Kurve sind kollektive Handlungen des Steuerwiderstands, bei denen die Steuerzahler die Grenzen der Besteuerung per Referendum setzen. ${ }^{179}$ Die Analyse fußt auf mikroökonomischen Entscheidungen und kann deshalb kollektive Bewegungen nicht darstellen. Einerseits könnte argumentiert werden, $\mathrm{da} B$ für die britische Steuerpolitik dies unerheblich ist, weil sich der verfassungsmäßige Rahmen für Steuerwiderstand praktisch auf ein parlamentarisches Mißtrauensvotum und die anschließende Abwahl der Regierung beschränkt, was im Betrachtungszeitraum nicht vorkam. ${ }^{180}$ Nichtsdestotrotz scheint ein breiter Konsens unter den OECD-Regierungen darin zu bestehen, daß dem Spielraum zur Konsolidierung der Staatsverschuldung mittels Steuererhöhungen deutliche politische Grenzen gesetzt sind. ${ }^{181}$ Auch im Vereinigten Königreich sind Steuererhöhungen in der politischen Diskussion praktisch Tabu. ${ }^{182}$ Es fragt sich, wo die politische Grenze der Besteuerung im Vereinigten Königreich liegt.

Bei zwei Steuerreformen der letzten Jahren hat ein kollektiver Widerstand sich in sozialer Unruhe geäußert, welche leicht als Grenze der Besteuerung zu erkennen war. Ein Beispiel war die 1990 umfassende Reform der Gemeindefinanzen. Eine örtliche kopfsteuerartige Abgabe, (Community Charge, auch „poll tax“ genannt) wurde eingeführt und die Kontrolle über die Höhe der Grundsteuer für von Unternehmen und Nichtansässigen bebaute Grundstücke von den Gemeinden an die

${ }^{177}$ In den USA ist 1986 der oberste Grenzsteuersatz von 50\% auf effektiv 33\% reduziert worden (US Tax Reform Act), mit der expliziten Begründung, daß das Aufkommen steigen würde. Die Steuereinnahmen sind aber zurückgegangen. Mit anderen Worten, die USA befanden sich auf dem aufsteigenden Ast der Laffer-Kurve.

178 Siehe Blundell und Walker (1986) und Blundell, Ham und Meghir (1987), sowie Dilnot et al. (1987). Einen Überblick der empirischen Literatur bietet Blundell (1992).

179 Ein bekanntes Beispiel war 1978 die kalifornische Volksabstimmung „Proposition 13“ zur Steuerbegrenzung, siehe hierzu Brennan und Buchanan (1979). In der Schweiz werden Steuererhöhungen häufig per Volksabstimmung abgelehnt, siehe Frey und Kirchgässner (1994), S. 226.

${ }^{180}$ Zur verfassungsmäßigen Rahmenbedingungen Großbritanniens siehe Bognador (1996).

${ }^{181}$ Oxley et al. (1990), S. 28.

182 So z. B. der Leitartikel in der Zeitung The Independent yom 249.96, S.13:3-631-75199-2 
Zentralregierung transferiert. Eine wesentliche Folge dieser Reformen war die Senkung des laufenden Einnahmenanteils von $50 \%$ auf $25 \%$, den die Gemeinden eigenständig finanzieren konnten. Somit wurde die Aufkommenselastizität der Steuereinnahmen der Gemeinden eingeschränkt: Der benötigte Anstieg der freigestaltbaren Steuer zur Finanzierung einer gegebenen zusätzlichen Ausgabe nimmt mit der Minderung der Bemessungsgrundlage zu. Infolge dessen erhoben diejenigen Gemeinden, die relative hohe Ausgaben für notwendig hielten, eine überdurchschnittlich hohe ,poll tax“. Verglichen mit dem vorigen System von Steuern auf der Nutzung von privatem Wohnraum mit vielen Erstattungsmöglichkeiten für Haushalte mit niedrigem Einkommen sahen sich die Haushalte in ärmeren Gemeinden mit entsprechend hohen Ausgaben mit einer enormen Steuererhöhung konfrontiert. ${ }^{183}$ Die Einführung der poll tax 1990 löste eine gewaltsame Protestwelle aus. Bis $\mathrm{zu} 60 \%$ der Steuererlöse konnten wegen Zahlungsverweigerung nicht eingenommen werden. Die Steuer wurde 1991 abgeschafft. ${ }^{184}$

Ein zweites Beispiel war 1994 die beabsichtigte Einführung der Mehrwertsteuer zum Normalsatz $(17,5 \%)$ auf Brennstoffe für den Haushaltsverbrauch. Vor allem aufgrund der Auswirkungen auf Heizkosten für Haushalte mit niedrigen Einkommen, z. B. für Rentner, wurde diese Reform ebenfalls von einem Großteil der Bevölkerung abgelehnt. Eine Oppositionskampagne wurde lanciert, die sich diesmal in Petitionen und Protestbriefen an das Parlament äußerte. Infolge der offensichtlichen Auflehnung der Bevölkerung lehnte das Parlament das entsprechende Finanzgesetz ab, und im folgenden Finanzgesetz wurde ein niedrigerer Steuertarif von $8 \%$ eingeführt. ${ }^{185}$

Aus diesen zwei Beispielen läßt sich schließen, daß politische Grenzen der Besteuerung auch für die Konservative Regierung bestehen. Anhand dieser Fälle lassen sich Bedingungen für die Auslösung von kollektiven Widerstand gegen Steuererhöhungen aufstellen: ${ }^{186}$ Wenn die Inzidenz einer Steuer transparent, regressiv und durch Substitutionshandlung unvermeidbar ist, wenn ihre Einführung eine sehr starke und plötzliche Veränderung der Steuerlast verursacht und wenn ihre Höhe in der Zukunft unberechenbar ist (wie im Falle der poll tax), sind einige Bedingungen für kollektiven Widerstand erfüllt. ${ }^{187}$

${ }^{183}$ Zur Geschichte der ,poll tax“ siehe auch Smith und Ridge (1990), insbesondere zu ihren Verteilungswirkungen, siehe Smith, S. (1991).

184 Smith (1994), S. 4.

${ }^{185}$ Financial Times 8.12.94 sowie 10.12.94.

186 Ähnlich bei Cullis und Jones (1992), die politische Kosten als Determinante des Steuersystems untersuchen, S. 452ff.

${ }^{187}$ Es sei in diesem Zusammenhang an Adam Smiths Grundprizipien der Besteuerung erinnert. Neben Effizienz und Einfachheit sind dies die Gerechtigkeit, daffndividyen as nearly as possible in 


\section{3) Grenzen der Besteuerung durch Steuerharmonisierung}

Der EG ist die Steuerharmonisierung unter ihren Mitgliedern seit ihrer Gründung im Jahre 1957 ein Anliegen. ${ }^{188}$ Mit der Verabschiedung der Einheitlichen Europäischen Akte von 1985 wurde im Rahmen des beabsichtigten Integrationsprozesses die Vereinheitlichung der Besteuerung konkret anvisiert. ${ }^{189}$ Die Beschlüsse im Maastrichter Vertrag vom Dezember 1991 zur Europäischen Währungsunion üben durch die Konvergenzkriterien der öffentlichen Finanzen einen weiteren Anpassungsdruck auf die finanzpolitischen Instrumente aus. ${ }^{190}$

Einen Ansatzpunkt, um die Bestrebungen der Steuerharmonisierung zu erklären und zu beurteilen, bietet die Theorie des Finanzföderalismus. ${ }^{191} \mathrm{Ihr}$ zufolge ist eine regional unterschiedliche Versorgung mit öffentlichen Gütern und deren Finanzierung Ausdruck regional unterschiedlicher Bedürfnisse und Präferenzen. Eine Konvergenz der Steuern und Ausgaben ist nicht notwendig, wenn die Güter und Produktionsfaktoren zwischen Regionen mobil sind. Die effiziente Versorgung mit öffentlichen Gütern findet durch „Abstimmung mit den Füßen“ statt: Mobile Wähler lassen sich dort nieder, wo die Bereitstellung öffentlicher Güter ihren Präferenzen entspricht. ${ }^{192}$ Ist die Mobilität der Wähler nur gering (beispielsweise wie in der EU aufgrund von Sprachgrenzen), dann kann die föderale Rollenverteilung durch die Mobilität von Unternehmungen und bestimmten Gruppen von Arbeitskräften stattfinden. ${ }^{193}$ Eine Steuerharmonisierung ist aus Sicht des Finanzföderalismus nicht notwendig und kann sogar wohlfahrtsmindernd wirken, wenn die Konsumentenvielfalt dadurch eingeschränkt wird.

proportion to their respective abilities" beitragen sollen, und die Sicherheit, d. h. die Steuerlast soll weder willkürlich noch unsicher für den Steuerzahler sein. Smith (1776), S. 825ff.

${ }^{188}$ Bereits der Römische Vertrag sah die Harmonisierung der indirekten Steuern der Mitgliedsländer vor. Das Neumark Komitee (1963) befaßte sich zum ersten Mal ausführlich mit Fragen der Steuerharmonisierung.

${ }^{189}$ Im Artikel 99 des EWG-Vertrags wird der Rat befugt, nach Beratung im Europäischen Parlament einstimmig über die Rechtsvorschriften der indirekten Besteuerung zu entscheiden. Artikel 100 enthält die Rechtsgrundlage zur Harmonisierung der direkten Steuern, die jedoch nur sehr allgemein formuliert ist. Europäische Gemeinschaften (1990), S. 6 f.

190 Wenn ein Land diese Kriterien nicht erfültt, kann auf Empfehlung der Kommission der Gemeinschaftsrat Reformvorschläge, einschließlich Steuerreformen machen, um Budgetstabilität wieder herzustellen. Haan et al. (1992), S. 5f. Von diesem Verfahren wird erhofft, daß ein fiskalischer Konvergenzdruck den Weg zur Europäischen Währungsunion ebnet. Gleichzeitig wird damit indirekt ein Weg geöffnet, die Steuerharmonisierung voranzutreiben.

191 Der klassische Beitrag stammt von Oates (1972).

192 Vgl. Tiebout (1956).

${ }^{193}$ Emerson et al. (1991), S. 144. 
Dieses einfache Ergebnis muß allerdings modifiziert werden, wenn Externalitäten zwischen den Hoheitsgebieten auftreten. Gehen positive „Spill-over“-Effekte der öffentlichen Einrichtungen (z. B. der Verkehrsinfrastruktur oder kultureller Einrichtungen) und negative Effekte (beispielsweise der Umweltverschmutzung) über die Grenzen der EU Mitgliedsländer hinaus, so wird das Gleichgewicht durch ein unzureichendes Angebot an öffentlichen Gütern (bzw. ein Überangebot an öffentlichen „Bads") gekennzeichnet. Externalitäten entstehen auch bei der Besteuerung. Unterschiedliche Besteuerung zwischen Hoheitsgebieten veranlaßt Verlagerungen der Bemessungsgrundlagen (d. h. Steuervermeidung), um die steuerliche Belastung zu senken. Die Preisänderungen, die aufgrund regional divergierender Steuersätze und Bemessungsgrundlagen entstehen, verzerren den allokativen Lenkungsmechanismus, setzen falsche Signale für die effiziente Allokation von Gütern und Faktoren im Wirtschaftsraum und lösen damit Güter- und Faktorbewegungen aus.

Daraus leitet sich der wesentliche Grund für die Bemühungen der Kommission um Steuerharmonisierung ab. ${ }^{194}$ Der Integrationsprozeß des Europäischen Binnenmarktes setzt die Mobilität von Gütern und Produktionsfaktoren zwischen den Mitgliedsländern voraus und damit die Ausnutzung von komparativen Kostenvorteilen aus Spezialisierung und Produktionsverteilung. Die allokativen Entscheidungen über Güter- und Faktorbewegungen sollen nicht durch Bewegungen aufgrund unterschiedlicher Steuersysteme der Mitgliedsländer konterkariert werden. Intragemeinschaftliche Verschiebungen der Steuereinnahmen machen zudem einen zentralen Umverteilungsmechanismus notwendig, um die entstandene Fehlallokation zu korrigieren. Es gibt noch weitere Argumente für Steuerharmonisierung: Unterschiedliche Steuersätze zwischen Hoheitsgebieten bieten einen Anreiz zur Steuervermeidung, der angesichts der Marktliberalisierung und dem Wegfall der Zollgrenzen kaum entgegenzuwirken ist. ${ }^{195}$ Längerfristig könnte die Anpassung der Steuerunterschiede durch Konkurrenz zwischen den Steuerhoheiten stattfinden. Die Steuerhoheiten würden die Steuersätze senken (beziehungsweise die Bemessungsgrundlagen schmälern) müssen, um mobile Güter und Faktoren anzulocken bzw. ihre Abwanderung zu verhindern. Es würde ein steuerlicher Wettlauf entstehen, der die Steuersätze auf ein suboptimales Niveau absenken würde.

Gegenwärtig scheint dieses Szenario jedoch in einer fernen Zukunft zu liegen. Die Steuersysteme der EU Mitgliedstaaten weisen noch erhebliche Unterschiede auf, wie ein Vergleich der wichtigsten Steuersätze in der Tabelle 1 zeigt.

${ }^{194}$ Kopits (1992), S. 3, Bovenberg und Horne (1992), S. 29f. sowie Westaway (1982), S. 82ff.

195 Deutlich wird dieses Argument bei der Besteuerung des mobilen Faktors Kapital. Bereits bei der Bekanntgabe einer Quellensteuer auf Kapitalerträge von 15\%, die 1989 in Deutschland eingeführt wurde, kam es zu einer enormen Kapitalflucht. Ausführlicher hierzu im Monatsbericht der Deutschen Bundesbank, (1994), S. 50ff. 
Tabelle 1: Ausgewählte Steuersätze in der EU (12) 1994

\begin{tabular}{|c|c|c|c|}
\hline & \multicolumn{3}{|c|}{ Einkommensteuer Körperschaftssteuer (\%) Mehrwertsteuer } \\
\hline & Spitzensatz (\%) & & Normalsatz (\%) \\
\hline Belgien & 55 & 39 & 19,5 \\
\hline Dänemark & 40 & 34 & 25 \\
\hline Deutschland & 53 & $30 / 45^{*}$ & 15 \\
\hline Frankreich & 56,8 & 33,3 & 18,6 \\
\hline Griechenland & 45 & $35+$ & 18 \\
\hline Irland & 48 & 43 & 21 \\
\hline Italien & 51 & 36 & 19 \\
\hline Luxemburg & 50 & 33 & 15 \\
\hline Niederlande & 60 & 35 & 17,5 \\
\hline Portugal & 40 & 36 & 17 \\
\hline Spanien & 56 & 35 & 15 \\
\hline UK & 40 & 33 & 17,5 \\
\hline
\end{tabular}

*Für ausgeschüttete (30\%) oder thesaurierte (45\%) Gewinne;

+ auf ausländische und an der Börse quotierte Unternehmen $40 \%$

Quelle: Bunz (Hrsg.)

Die geringen Harmonisierungserfolge haben vor allem einen politischen Hintergrund. ${ }^{196}$ Fiskalpolitische Entscheidungen werden nach dem Einstimmigkeitsprinzip gefällt. Somit können einzelne EU Mitgliedsländer, die sich in ihrer fiskalpolitischen Autonomie bedroht fühlen oder plötzliche Einnahmeausfälle infolge einer Steuerharmonisierung befürchten müssen, Maßnahmen zur Steuerharmonisierung verhindern. ${ }^{197}$ Bisher wurde Harmonisierung nur bei den indirekten Steuern vereinbart. Die Mehrwertsteuer wurde im Laufe der 80er Jahre in allen Ländern der EU eingeführt und ihre Bemessungsgrundlage weitgehend harmonisiert. Eine Übergangsregelung bis Ende 1996 wurde getroffen, wonach ab 1. Januar 1993 das Ursprungslandprinzip mit einem Normalsteuersatz von mindestens $15 \%$ und einem bis zwei ermäßigten Sätzen von mindestens 5\% vereinbart wurden. 1997 soll das Bestimmungslandprinzip eingeführt werden. ${ }^{198}$ Diese Lösung wird zu einem Steuerwettbewerb führen bei dem, wie Lockwood et al. (1994), S. 9 schreiben, „each country [tries] to undercut its neighbour to attract cross-border shoppers and so divert tax revenues to itself.“ Die Mitgliedsländer werden gezwungen, die indirekten Steuersätze auf ein minimales Niveau zu harmonisieren.

Eine einheitliche Quellensteuer auf Kapitalerträge von $15 \%$ wurde von der Kommission vorgeschlagen, um der Steuervermeidung zwischen EU-Ländern Einhalt

196 Auf die ökonomischen Argumente gegen eine ,aktive“ Steuerharmonisierung in der EU wird hier nicht eingegangen, siehe McLure (1986), Giovannini (1989), Eichengreen (1990) und Spahn und Kaiser (1991).

${ }^{197}$ Europäische Gemeinschaften (1990), S. 7.

198 Lockwood et al. (1994). 
zu gebieten. ${ }^{199} \mathrm{Da}$ jedoch die Kapitalmärkte weltweit liberalisiert werden, kann eine Harmonisierung innerhalb der EU die Kapitalflucht aus der EU nicht wirksam verhindern. Es besteht weitgehende Einigkeit, daß die vollkommene Integration von Kapitalmärkten die Abschaffung von nationalen Steuern auf Kapital bedeuten wird. ${ }^{200}$ Langjährige Harmonisierungsbemühungen im Bereich der Körperschaftssteuer sind bislang weitgehend erfolglos geblieben. Die Kommission gab in April $1990 \mathrm{ihr}$ seit 1975 bestehendes Harmonisierungsziel für die Besteuerung von Körperschaften auf. ${ }^{201}$ Schließlich wird bei den Einkommensteuern und Sozialversicherungsbeiträgen noch kein aktiver Harmonisierungsbedarf gesehen.

Selbst wenn vom Status Quo ausgegangen wird (d. h. keine weitere aktive Steuerharmonisierung stattfindet), gibt es starke historische Beweise, daß zunehmende Integration einen spontanen und tendenziell zu niedrigeren Steuern gerichteten Konvergenzdruck auf die nationalen Steuersysteme ausübt. ${ }^{202}$ Mit zunehmender wirtschaftlicher Integration sinken die Möglichkeiten, einen gegebenen Steuersatz über die Sätze der konkurrierenden Steuerhoheiten zu setzen, weil in Abhängigkeit ihrer Mobilität die Faktoren in ein anderes Hoheitsgebiet ziehen, wo ihre Besteuerung niedriger ausfällt.

In bezug auf die Elastizität der Steuereinnahmen wird eine solche Entwicklung den Spielraum für die einzelnen Mitgliedsländer einschränken. ${ }^{203}$ Der Steuerwettbewerb bildet im EU-Binnenmarkt eine dritte Grenze der Besteuerung. Der im vorigen Abschnitt dargestellte negative Zusammenhang zwischen dem Steuersatz und dem Aufkommen verstärkt sich noch, d. h. die Steuersatzelastizität des Einkommens $(\tau / Y * d Y / d \tau)$ steigt. $^{204}$

199 Spahn und Kaiser (1991), S. $28 \mathrm{ff}$.

200 Weichenrieder (1996), S. 37 und die dort zitierte Literatur.

${ }^{201}$ Gardner (1992), S. 58ff.

202 Siehe vor allem die Studie der OECD-Länder in Hagemann, Jones und Montador (1988). Eichengreen (1990) untersucht die Konvergenz der Steuersätze in den USA. Im Gegensatz zur geplanten und zunehmenden Einflußnahme der Union in Europa entscheiden die Bundesstaaten ihre Fiskalpolitik ohne Zuweisungen des Zentralstaates. Nichtsdestotrotz hat eine spürbare spontane Harmonisierung der Steuersätze stattgefunden. Die Variabilität der Steuersätze ist ungefähr $\mathbf{4 0 \%}$ geringer zwischen den amerikanischen Bundesstaaten als zwischen den damaligen 12 EC-Mitgliedern.

${ }^{203}$ Hierzu Haan et al. (1992).

204 Prinzipiell gelten analoge Argumente für Maßnahmen zur Aufkommensteigerung, welche die Bemessungsgrundlage breiter als die der konkurrierenden Steuersysteme gestalten. Effizienzverbesserungen des Steuersystems können möglicherweise das Aufkommen erhöhen, wobei die Abwanderungen der Bemessungsgrundlage $\mathrm{zu}$ ineffizienten Hoheitsgebieten auch möglich ist. Eine effektive Steuerharmonisierung muß alle drei Gestaltungsmerkmale berücksichtigen. Kay und King (1990), S. 218. Zur Vereinfachung beschränkt sich die Analyse im wesentlichen auf die Steuersätze, welche als wichtigstes Gestaltungsmerkmal für den Steugzwettbewerb geltegn.2 
Analog zum Substitutionseffekt der Laffer-Kurve wird das Steueraufkommen sinken, wenn der Wettbewerbseffekt einer Steuersatzerhöhung die Budget- und Einkommenseffekte überwiegt. Der Wettbewerbseffekt tritt auf, wenn der einheimische Steuersatz über den konkurrierenden Sätzen liegt. Seine Stärke hängt von der Elastizität des Steueraufkommens in bezug auf die Differenz zwischen dem einheimischen und dem durchschnittlichen EU-Steuersatz ab. ${ }^{205}$ Diese Elastizität dürfte sich im Zuge der Integration erhöhen. Es wird zunehmend schwierig, das Steueraufkommen durch Gestaltungsmerkmale zu erhöhen, die von den EUDurchschnittswerten abweichen. Somit lassen sich die durchschnittlichen Steuersätze der EU als dritte Grenze der Besteuerung aufstellen.

\section{Strategie III: Eine Senkung der öffentlichen Ausgaben}

In diesem Abschnitt werden Möglichkeiten untersucht, die Verschuldung durch eine Senkung der öffentlichen Ausgaben zu verringern. Die Diskussion über die Höhe der öffentlichen Ausgaben führt häufig zur weitreichenden Frage des politisch und ökonomisch erwünschten Staatseinflusses. Dieser Aspekt läßt sich hier in diesem Rahmen nicht behandeln und wird ausgeklammert. Das heißt, es geht hier um die unmittelbare budgetpolitische Frage, wie die Staatsquote ${ }^{206}$ gesenkt werden kann. Die nachfolgenden Erörterungen greifen auf Argumente zurück, welche aus den Theorien zum Wachstum der Staatsausgaben gewonnen wurden. Darüber hinaus wird versucht, die politische Akzeptanz einer gegebenen Sparmaßnahme bei den Wählern zu berücksichtigen.

\section{1) Theorien zur Entwicklung der Staatsausgaben}

Es wird kaum bestritten, daß zumindest in diesem Jahrhundert der öffentliche Sektor gemessen als Staatsanteil am Sozialprodukt in allen Industrieländern prak-

${ }^{205}$ Keen (1987) zeigt, daß Steuerharmonisierung zu den gewichteten EG Durchschnittssteuersätzen hin einen Wohlfahrtsgewinn für die EG bedeuten würde.

${ }^{206}$ Die Staatsquote umfaßt die nominalen Staatskäufe sowie die Transferzahlungen als Anteil am Sozialprodukt. Die Schwächen dieses Indikators für den Umfang der staatlichen Tätigkeit sind bekannt. Zur Definitions- und Meßproblematik siehe Lybeck (1986), S. 21 ff. sowie Gemmell (1993), S. $1 \mathrm{ff}$. Für die folgende budgetfinanzielle Analyse erscheint die Staatsquote als Kennzahl gerechtfertigt, weil sie die staatliche Inanspruchnahme der volkswirtschaftlichen Ressourcen bemißt, deren Finanzierung entsprechend der staatlichen Budgetbeschränkung durch die staatlichen Einnahmen gewährleistet wird. 
tisch ununterbrochen zugenommen hat. ${ }^{207}$ Die resultierende Diskussion über die Gründe für dieses säkulare Wachstum hat zu einer Fülle von Theorien geführt, ${ }^{208}$ welche als Grundlage für diese Überlegungen dienen können. In Anlehnung an Mueller (1989) werden im folgenden zwei verschiedenen Konzepte des Staates unterschieden, aus denen nachfrage- und angebotsbedingte Theorien abgeleitet werden können. ${ }^{209}$ Den nachfragebedingten Theorien liegt die klassische Auffassung des Staates zugrunde, wonach dieser als Transmissionsmechanismus existiert, um den vom Volk geäußerten Willen in die Wirklichkeit umzusetzen. Das Ausmaß der Staatstätigkeit wird allein durch die Nachfrage des Volkes bestimmt. Das staatliche Angebot verhält sich vollkommen elastisch und paßt sich dieser Nachfrage an. Dagegen stellen die angebotsbedingten Theorien diese Auffassung des passiven Staates in Frage. Danach sind die wirtschaftlichen Akteure hinter dem staatlichen Angebot, beispielsweise die Politiker oder die Bürokraten, nicht nur um das Wohl der Wähler besorgt, sondern verfolgen auch ihre eigenen Interessen. Die angebotsorientierten Theorien versuchen diesen Tatbestand zu berücksichtigen.

\section{2) Nachfragebedingte Theorien}

In einem demokratischen Staat mit marktwirtschaftlicher Prägung wird eine Regierung sich nur im Amt halten bzw. wiedergewählt werden, wenn sie das Angebot an staatlichen Leistungen zumindest generell nach den Wünschen einer Mehrheit der Wähler richtet. ${ }^{210}$ Den nachfragebedingten Theorien zufolge spiegelt das Wachstum der Staatsquote die Präferenzen der Wähler wider. Staatliche Leistungen können entweder durch die Bereitstellung von Gütern und Dienstleistungen oder die Gewährung von Transfers erfolgen.

\section{a) Der Staat im Strukturwandel}

Bereits im letzten Jahrhundert prognostizierte Adolf Wagner $(1883,1892)$ wachsende Staatsausgaben. Die Begründung seiner Voraussage ist jedoch umstritten und wird unterschiedlich gedeutet. ${ }^{211}$ Eine der Hypothesen postuliert einen strukturellen Wandel als Ursache der zunehmenden Staatstätigkeit. Erstens haben demogra-

\footnotetext{
${ }^{207}$ Dieses Wachstum dokumentiert z. B. Saunders (1993).

208 Überblicke bieten z. B. Mueller (1989), Lybeck (1988), Pelzman (1980) und Peacock (1979).

209 Eine ähnliche Klassifikation geht auf Tarschys (1975), S. 13ff. zurück und wurde von anderen Autoren übernommen, siehe z. B. Burton, (1985) oder Lybeck (1986).

${ }^{210}$ Hierzu Downs (1957), S. $51 \mathrm{ff}$.

${ }^{211}$ Hierzu Lybeck (1988), S. 31. 
phische, soziologische und technologische Veränderungen der Gesellschaft zu Präferenzenänderungen bei den Wählern geführt und die Nachfrage zugunsten öffentlich bereitgestellter Güter erhöht. Die Entwicklung von der agrarischen zur industriellen und anschließend zur post-industriellen Gesellschaft brachte die moderne Arbeitsteilung mit sich, welche erhöhte Spezialisierung und eine Zunahme von Markttransaktionen verursachte. ${ }^{212}$ Diese Veränderungen erhöhten einerseits die Nachfrage nach reinen öffentlichen Gütern im Sinne des „minimal state“ von Buchanan (1975), wie z. B. Gesetze, Gerichte und andere Dienste der öffentlichen Ordnung, und andererseits nach Vorsorgeleistungen des Wohlfahrtsstaats. Zudem brachten sie eine Wohlstandsverbesserung im Sinne eines Anstiegs des realen ProKopf Einkommens sowie der durchschnittlichen Lebenserwartung mit sich, welche den Bedarf nach einkommmensabhängigen Gütern und Dienstleistungen erhöhte. Nach Wagner [(1883), S. 76f.; (1892), S. 904ff] haben viele öffentlich bereitgestellte Güter und Dienstleistungen - insbesondere nennt er Gesundheits- und Erziehungseinrichtungen - eine Einkommenselastizität größer als eins, d. h. mit steigendem Einkommen steigt die Nachfrage nach diesen Gütern überproportional an.

Nach der Wagnerschen Hypothese des Strukturwandels mußte der Anstieg der Staatsausgaben vorwiegend bei den traditionellen allokativen Aufgaben stattgefunden haben, welche Kollektivcharakter haben, (z. B. Militär, Infrastruktur, Justiz und Polizei), sowie bei Luxusgütern und -dienstleistungen (Erziehung, Gesundheit und Kultur). Allerdings erklärt Wagners Hypothese nicht, warum solche Leistungen von staatlicher statt privater Hand bereitgestellt werden, obwohl viele keine reinen öffentlichen Güter ${ }^{213}$ sind. Aus polit-ökonomischer Sicht ist anzumerken, $\mathrm{da} ß$ entsprechend dieser Interpretation der Ausgabenansteig von den Wählern als gewollt unterstellt wird. Demzufolge ist es in einer Demokratie weder wünschenswert noch dauerhaft möglich, diese Entwicklung in Ausgabensenkungen umzukehren.

\section{b) Der Staat im Funktionswandel}

Eine zweite Interpretation der Wagnerschen Hypothese betont den Staat im Funktionswandel. Der Staat hat sich mit der Entstehung des Wohlfahrtstaats vermehrt Umverteilungsaufgaben gewidmet. Diese Entwicklung kommt vor allem durch erhöhte Transfer- und Subventionszahlungen, sowie durch öffentlich bereitgestellte Güter und Dienstleistungen mit Verteilungscharakter zum Ausdruck, z. B. für Gesundheit und Bildung.

212 Ein neuerer Ansatz in diesem Sinne betont die erhöhten Transaktionskosten einer Marktwirtschaft mit zunehmender Spezialisierung, North (1985).

${ }^{213}$ Reine öffentliche Güter sind durch Nicht-Ausschließbarkeit und Nicht-Rivalität im Konsum gekennzeichnet. 
Bereits Alexis de Tocqueville (1835) sagte in „De la démocratie en Amérique“ ein Wachstum der Staatsausgaben voraus, wenn das Wahlrecht erweitert wird und/oder die Einkommens- und Vermögensverteilung ungleichmäßiger wird, weil er deswegen eine größere Nachfrage nach staatlichen Verteilungsaktivitäten erwartete. ${ }^{214}$ Meltzer und Richard (1981) haben diesen Gedanken aufgegriffen und eine verteilungspolitische Erklärung der wachsenden Staatsausgaben entwickelt. Weil der Anstieg der Staatsausgaben in den meisten Ländern bei derartigen Leistungen stattgefunden hat, ${ }^{215}$ wird auf dieses Modell ausführlich eingegangen.

Seinen Ausgangspunkt bilden zwei Annahmen. Erstens sind bei einer gegebenen Wahlberechtigungsquote die Stimmrechte gleichmäßig auf die Stimmberechtigten verteilt, während die Einkommensverteilung unter den Stimmberechtigten ungleichmäßig verteilt ist. Zweitens ist das einzige Ziel der staatlichen Tätigkeit eine breite Nivellierung der personellen Einkommensverteilung mittels einkommensabhängiger Transferzahlungen, finanziert durch eine proportionale Einkommensteuer auf das Arbeitseinkommen. ${ }^{216}$ Aufbauend auf dem Medianwählermodell [Hotelling (1929) und Downs (1957) $]^{217}$ wird ein Umverteilungsprozeß beschrieben, wo die Wähler in Abhängigkeit ihrer Einkommensmöglichkeiten (welche aufgrund zufälliger unterschiedlicher Produktivitäten ungleich sind) über das gewünschte Niveau von Transferzahlungen abstimmen. Übersteigt bei der Einkommensverteilung das durchschnittliche Einkommen (Mittelwert) das Medianeinkommen, wie dies bei der normalerweise rechtsschiefen Einkommensverteilung der Fall ist, ${ }^{218}$ so stimmen die Bezieher (relativ) geringer Einkommen für einen Ansteig der Umverteilungsmaßnahmen, finanziert durch eine Besteuerung der hohen Einkommensbezieher. Der Anteil des gesamten Wählereinkommens, der über dem Medianeinkommen liegt, stellt das zur Umverteilung verfügbare Einkommen dar. Die Niedrigeinkommensbezieher und der entscheidende Medianwähler können dieses Einkommen durch ihr Stimmverhalten an sich umverteilen. Der Anreiz, dies zu tun, steigt, wenn die verfügbaren Mittel zur Umverteilung (d. h. der Anteil des gesamten Wählereinkommens, welche über dem Medianwählereinkommen liegt) zunehmen. Historisch ist dies eingetreten, als das Wahlrecht auf die ärmeren Teile der Bevölkerung ausgedehnt wurde. Der Druck zur Umverteilung und folglich zur zunehmenden verteilungspolitischen Staatstätigkeit erhöht sich ebenfalls, wenn die Ungleichheit der Einkommensverteilung zunimmt. Der Grundgedanke des Modells läßt sich in Abbildung 8 in stark vereinfachter Form veranschaulichen.

214 De Tocqueville (1835), Kap. XII, Teil I (wieder abgedruckt, 1965) S. 150.

215 Siehe die in der Fußnote 208 zitierten Literaturüberblicke.

${ }^{216}$ Vereinfachend wird von anderen staatlichen Aktivitäten abstrahiert. Die Ausgaben werden nur für Transferzahlungen verwendet.

${ }^{217}$ Für eine anschauliche Darstellung siehe Mueller (1989), Kap. 10.

${ }^{218}$ Siehe z. B. Atkinson (1983), S. 13. 


\section{Abbildung 8: Steigende Staatsausgaben durch Verteilungspolitik}

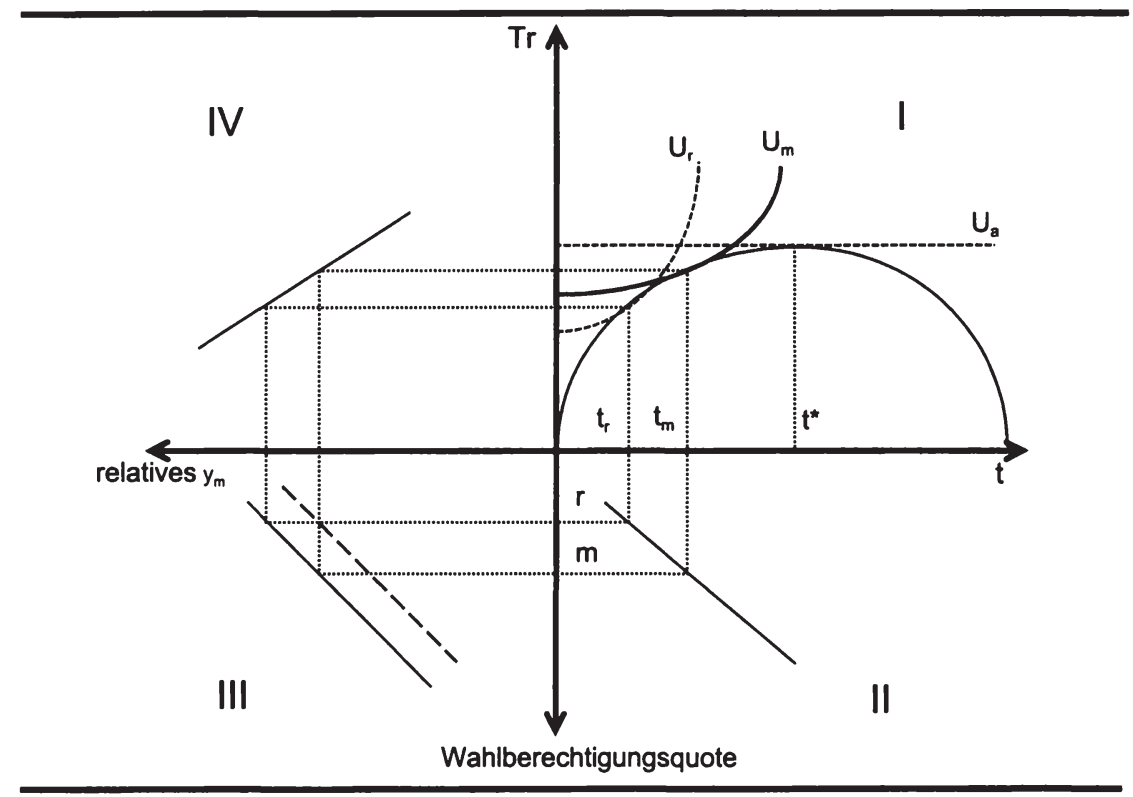

Rechts oben im Quadrant I wird die Steueraufkommensfunktion in Abhängigkeit vom Grenz- bzw. Durchschnittsteuersatz abgebildet (vgl. Abbildung 6 im vorigen Abschnitt). Das gesamte Steueraufkommen bestimmt die zur Umverteilung verfügbaren Transfermittel. Der Laffer-Zusammenhang setzt dem Steueraufkommen eine Obergrenze (bei $\left.t^{*}\right) .{ }^{219}$ Der Nutzen der Wähler hängt entsprechend dem individuellen Maximierungskalkül vom Steuersatz und von den Transferleistungen ab. Steuerpflichtige Wähler haben positiv geneigte Indifferenzkurven, wie z. B. $U_{r}$ und $\mathrm{U}_{\mathrm{m}}{ }^{220}$ Die reichen Wähler stimmen für relativ niedrige Steuersätze und Transferleistungen, wie die steilverlaufende Kurve $\left(U_{r}\right)$ der Wähler einer hohen Einkommensgruppe verdeutlicht. Die ärmste Wählergruppe erhält kein steuerpflichtiges Einkommen. Sie wird unabhängig vom Steuersatz die maximalen Transferleistungen bevorzugen. Die Nutzenfunktion $\left(U_{a}\right)$ für diese Bevölkerungsgruppe verläuft deswegen waagerecht. Im zweiten Quadrant ist der Zusammenhang zwischen Wahlberechtigungsquote und Steuersatz aufgetragen. Wird das Wahlrecht auf neue Bevölkerungsgruppen ausgedehnt, können diese über das Niveau der Besteuerung

${ }^{219}$ Somit wird unterstellt, daß die Wähler rational sind und die Konfliktbeziehung zwischen Effizienz und Gerechtigkeit verstehen.

220 Solche aggregierten Nutzenfunktionen unterstellen homogene Präferenzen innerhalb einer Einkommensgruppe. 
und damit über die Ausgaben mitentscheiden. Weil neue Wähler annahmegemäß zu relativ ärmeren Einkommensgruppen gehören, sind sie für höhere Steuern zur Finanzierung zusätzlicher Ausgaben. Daraus folgt: Je höher die Wahlberechtigungsquote, desto höher die Steuersätze. Im dritten der Zusammenhang zwischen dem Ausmaß der Wahlberechtigung und dem relativen Einkommen des Medianwählers. ${ }^{221}$ Der vierte Quadrant bildet das Verhältnis zwischen relativem Medianwählereinkommen und gewünschten Transferzahlungen ab.

Ist nur der reiche Teil der erwachsenen Bevölkerung wahlberechtigt (auf der Höhe von $r$ im Quadrant II), dann ist das Medianwählereinkommen relativ hoch. Der Medianwähler bei dieser Gruppe wird ein relativ niedriges Steuer- und Ausgabenniveau $\left(t_{T}\right)$ bestimmen. Wenn das Wahlrecht auf die gesamte erwachsene Bevölkerung ausgedehnt wird (vom Punkt $r$ auf Punkt $m$ ), dann sind auch die ärmeren Bevölkerungsmitglieder wahlberechtigt und das relative Medianwählereinkommen nimmt ab (Quadrant III). Diese Bevölkerungsgruppe wünscht sich höhere Transferzahlungen (Quadrant IV). Die flacher verlaufende Nutzenfunktion $\left(U_{m}\right)$ zeigt die Ausgaben-Steuer-Präferenzen des neuen Medianwählers, deren Verwirklichung einen Anstieg des Steuersatzes notwendig macht (Quadrant II). Wenn bei unveränderter Wahlberechtigung die Einkommensverteilung der Wähler ungleichmäßiger wird, sinkt das relative Medianwählereinkommen und das gewünschte Ausgabenniveau steigt. Diese Entwicklung wird durch eine parallele Rechtsverschiebung der Funktion im Quadrant III abgebildet. ${ }^{22}$

Das personelle Umverteilungsmodell vom Meltzer und Richard (1981) stellt die zunehmende Bedeutung des distributiven Vorsorge- und Wohlfahrtstaats in den Mittelpunkt der Betrachtung. Es prognostiziert einen säkulären Anstieg der Staatsausgaben in Form von interpersonellen Transferzahlungen und zeigt, wie in einer Demokratie eine Koalition der ärmeren Gesellschaftsmitglieder Umverteilungsmaßnahmen zu sich hin erzwingen kann. Interessant am Ansatz ist, wie die Beziehung zwischen Steueraufkommen und Ausgabenhöhe explizit berücksichtigt wird: Die Wähler beziehen die Effizienzverluste der Einnahmefinanzierung in ihre Entscheidung über die Umverteilungsausgaben ein. Wie im ersten Kapital, Abschnitt I.2.f, argumentiert wurde, läßt sich die budgetpolitische Finanzierungsfrage nicht vernachläßigen. Die Berücksichtigung der Ausgabenfinanzierung durch den LafferZusammenhang hat erstens zur Folge, daß den Ausgaben technische Grenzen durch die Steuereinnahmen gesetzt werden. Zweitens müssen die Wähler die Effizienz-

221 Implizit ist dies der in der Lorenzkurze dargestellte Ordnung der kumulierten Anteile der Einkommen auf den kumulierten Anteilen der betreffenden Einkommensbezieher bzw. Wähler.

222 Die Form und Lage der jeweiligen Funktionen im Diagramm sind zwecks Anschaulichkeit gewählt worden. Selbstverständlich sind anders verlaufende Zusammenhänge oder Bewegungen denkbar. 
verluste bei hohen Steuersätzen abwägen, weshalb es im Modell nicht notwendigerweise zu einer vollkommenen Gleichverteilung des Einkommens kommt. ${ }^{223}$

Im Hinblick auf die Möglichkeiten für Ausgabenkürzungen lassen die Prämissen des Modells über die Wahlregeln und Ausgabenfinanzierung keine Ausgabensenkungen rechtfertigen. Zum einen widersprächen Ausgabensenkungen den Wünschen der rationalen Wählermehrheit und zum anderen wird die wachsende Staatstätigkeit durch das Steueraufkommen beschränkt. Allerdings gilt das zweite Argument nicht mehr unbedingt, wenn der Staat sich anderer Finanzierungsquellen, wie z. B. der öffentlichen Verschuldung, bedienen kann. Dann können kurzfristig die Ausgaben schneller als die Steuereinnahmen steigen. Nach Meltzer und Richard (1981) S. 927 wurde von dieser Quelle im 20. Jahrhundert erschöpfend Gebrauch gemacht, wie die Entwicklung der Staatsverschuldung gezeigt hat. Die Zinszahlungen des Staatskredits schränken jedoch die Verteilungsausgaben ein und haben zudem eigene Verteilungs- und Allokationswirkungen, welche die Ausgabenpolitik zumindest teilweise konterkarieren, (Kapitel I, Abschnitt IV.2). Ähnlich verhält es sich mit der Geldfinanzierung, wenn sie einen Inflationsanstieg mit eigenen Verteilungs- und Allokationsfolgen verursacht. ${ }^{224}$ Das Maximierungskalkül der Wähler unter Berücksichtigung anderer Finanzierungsquellen als die proportionale Einkommensteuer wird erheblich komplizierter. $\mathrm{Ob}$ es für den Medianwähler unter diesen Umständen immer noch optimal ist, Ausgabenerhöhungen zwecks Umverteilung zu erzwingen, läßt sich nicht a priori sagen. ${ }^{225}$ Es bleibt damit unklar, wie die Staatsausgaben sich entwickeln, wenn der Staat nicht auf die proportionale Steuerfinanzierung beschränkt ist, sondern die Last durch Verschuldung verschieben kann.

Die Ausgabenpolitik im Modell beschränkt sich ausschließlich auf Pauschaltransferzahlungen von den Reichen zu den Armen mit dem Ziel einer breiten Nivellierung des personellen Einkommens. Andere Verteilungsziele oder -strategien werden nicht berücksichtigt. Das Modell ließe sich zwar auch auf die Bildung anderer mehrheitlicher Koalitionen ausdehnen und damit mit intertemporalen Verteilungselementen, wie z. B. Verteilungspolitik zugunsten älterer und jüngerer Gesellschaftsmitglieder, erweitern. ${ }^{226}$ Aber der Vielfalt an verteilungspolitischen Zielen

${ }^{223}$ Bereits Downs (1957), S. 200 machte darauf aufmerksam, daß potentielle Umverteilungsgewinner bei ihren politischen Entscheidungen die Anreizeffekte der Besteuerung berücksichtigen. Lindbeck (1985), S. 320 schlägt eine Erweiterung des Modells vor, welche Unsicherheit über das zukünftige Einkommen berücksichtigt. Für reiche Wähler haben die Transferleistungen eine Versicherungsfunktion. Arme Wähler tolerieren gewisse Einkommensunterschiede, weil sie hoffen, künftig in eine höhere Einkommensklasse zu kommen.

${ }^{224} \mathrm{Zu}$ den Wirkungen der Inflation siehe Fischer und Modigliani (1978).

225 Vgl. hierzu Peacock (1983) S. 11f.

${ }^{226}$ Fellner (1983), S. 28f. 
und Auswirkungen der staatlichen Ausgabentätigkeit wird es nicht gerecht. ${ }^{227}$ Lindbeck (1985) nennt neben vertikalen Maßnahmen bei der personellen Verteilung und bei versicherungsartigen oder lebenszyklischen Umverteilungen zwei weitere verteilungspolitische Ziele: Erstens breite horizontale Umverteilungen zwischen funktionalen Einkommensklassen oder sozio-ökonomischen Sektoren, wie z. B. zugunsten der Landwirtschaft oder der Bergbauindustrie und zweitens fragmentierte horizontale Umverteilung zwischen unterschiedlichen Gruppen in einer hoch differenzierten sozio-ökonomischen Gesellschaftsstruktur. Pelzman (1980) S. $232 \mathrm{f}$. zeigt, wie die Umverteilungspolitik die Bildung von Gruppen fördert, die nicht durch ihre Einkommenshöhe, sondern durch ihre Unterstützung für bestimmte Ausgabenprogramme gebildet werden. Wenn die Verteilungspolitik nicht ausschließlich der interpersonellen oder intertemporalen Einkommensnivellierung dient, sondern einzelne Gruppen bevorzugt, dann sind zusätzliche Erklärungen für Ausgabenentscheidungen notwendig. Hierzu bieten sich die Theorien über Interessengruppen an.

\section{c) Theorien über das Verhalten von Interessengruppen}

Die Auswirkungen von Interessengruppen auf das staatliche Ausgabenniveau lassen sich als eine Nachfrageverzerrung interpretieren, weil Entscheidungen über die Ausgaben nicht nach der einfachen Mehrheitsregel gefällt werden, wie z. B. in Medianwähleransätzen unterstellt wird, sondern von partikulären Gruppen, welche zur Minderheit gehören können. Die grundlegenden Beiträge gehen auf Tullock (1959) und Olson (1965), (1982) zurück. Ausgangspunkt ist der Umstand, daß in einer repräsentativen Demokratie Individuen Interessengruppen bilden, um ihre Anliegen im politischen Entscheidungsprozeß effektiver durchzusetzen. Dabei haben kleine Gruppen mehr Erfolg, weil ihre Organisationskosten niedriger ausfallen und sie „Trittbrettfahrer-Verhalten" unter den Nutznießern effektiver unterbinden können. ${ }^{228}$ Haben ihre Anliegen auch positive Externalitäten, insbesondere für die Allgemeinheit, erhöhen sich ihre Erfolgschancen zusätzlich. ${ }^{229}$ Ihr Interesse in bezug auf die Staatsleistungen gilt für gruppenspezifische Vorteile. So werden sich ihre Bemühungen nicht auf rein öffentliche Kollektivgüter beziehen, sondern auf meritorische Güter mit Verteilungscharakter oder gesonderte Transferleistungen

${ }^{227}$ Umverteilungen werden nicht nur durch die Staatsausgaben, sondern auch durch die Steuerpolitik, Regulierungen oder Schutzmaßnahmen, wie z. B. eine Preis- oder Wechselkurspolitik oder sonstigen Protektionismus, erreicht. Hiervon soll jedoch an dieser Stelle abstrahiert werden.

${ }^{228}$ Olson (1965), S. 9-16 und 22-65.

${ }^{229}$ Becker (1983), S. 384. Nach dieser These haben beispielsweise Beschäftigte im Erziehungswesen mehr Durchsetzungsmacht als in der Druckindustrie 
und Subventionen. ${ }^{230}$ Diese werden von Politikern gewährt, die im Gegenzug implizit oder explizit künftige Wahlunterstützung erwarten. Dabei ist das aktive Auftreten einer Interessengruppe nicht Voraussetzung für die Gunst der Politiker, sondern lediglich für ihre Identifizierbarkeit als Wählergruppe. ${ }^{231}$ Finanziert werden die resultierenden Mehrausgaben von den allgemeinen Steuerzahlern, welche als größere Gruppe höhere Organisations- und Informationskosten für politische Aktivitäten zu tragen haben und deswegen weniger effektiv Widerstand gegen die kleinere Interessengemeinschaft leisten.

Bislang hat es trotz der grundlegenden Arbeiten von Tullock (1959) und Olson (1965), (1982) nur wenige Versuche ${ }^{232}$ gegeben, die genauen Wirkungsweisen und Auswirkungen von Interessengruppen auf die Staatsausgaben zu beschreiben oder empirisch zu ermitteln. Es überrascht nicht, daß verteilungspolitische Anliegen die Interessengruppen in der Regel motivieren, weil sie gruppenspezifische Vorteile suchen. Zu ihrer Identität betont Rowley (1979) die Rolle von Gewerkschaften, den Staatsbediensteten und professionellen Organisationen, wie z. B. Ärzteverbänden im Vereinigten Königreich. Budd (1979) sieht Bürokraten, insbesondere im britischen Finanzministerium als eine starke Interessengruppe an. Dies zeigt, daß Interessengruppen nicht nur Wähler, die Staatsleistungen nachfragen, sondern auch Staatsangestellte sein können, die an einem überhöhten staatlichen Angebot interessiert sind. Somit paßt sich das Angebot nicht einfach der - wie auch immer aggregierten - Nachfrage an, sondern übt auch einen Einfluß auf die Höhe der Staatsausgaben aus. ${ }^{233}$ Derartige Erklärungen sollen nun im folgenden kurz behandelt werden.

\section{3) Angebotsbedingte Theorien}

Die Grundannahme dieser Theorien besteht darin, daß der Staat als Anbieter öffentlicher Leistungen nicht einfach die Wählerwünsche umsetzt, sondern in irgendeiner Weise das Resultat aktiv beeinflußt. Die Theorien betonen, daß Staatsbedien-

${ }^{230}$ Nach Buchanan und Tullock (1967), S. 286ff. ist die Einführung von meritorischen Gütern im staatlichen Leistungsangebot auf die Lobbyarbeit von Interessengruppen zurückzuführen.

${ }^{231}$ Lindbeck (1985), S. 314.

232 Mueller (1989), S. 334. Unter einigen neueren Arbeiten sind Becker (1985), North (1985), Mueller und Murrell (1985), Lybeck (1986), Renaud und van Winden (1988) und Borooah (1988) zu nennen.

233 Die Annahme eines benevolenten Staats ist auch deswegen offensichtlich unvollständig, weil viele staatliche Maßnahmen - etwa Subventionen und Eintrittsbarrieren in bestimmten Industriezweigen, Zölle und andere Regulierungen mit keiner traditionellen sozialen Wohlfahrtsfunktion vereinbar sind, Becker (1985), S. 329. 
stete eigene Interessen haben. Verschiedenartige Systemschwächen und Ineffizienzen ermöglichen es ihnen, sich über die Wünsche der Wähler zu stellen und das Angebot an Staatsleistungen diesen Eigeninteressen anzupassen. Folglich stellt die Bindung an den Auftrag der Wähler eine nur bedingt wirkungsvolle Beschränkung gegen die autonome Staatstätigkeit dar.

\section{a) Steigende Stückkosten der öffentlich bereitgestellten Güter}

Die Staatsquote wird in nominalen Werten gemessen. Wenn ihre Zähler und Nenner mit unterschiedlichen Indizes deflationiert werden, ist es denkbar, daß der Anstieg der ermittelten realen nicht so stark ausfallt wie diejenige der nominalen Staatsquote. Auch ein Anstieg der nominalen Quote ist mit einer Abnahme der realen Quote kompatibel. ${ }^{234}$ Dieses Phänomen wird „relativer Preiseffekt“235 genannt. Beim gesamtwirtschaftlichen Inflationsindex, der für das Sozialprodukt verwendet wird, sind Qualitätsverbesserungen bei Gütern sowie der Produktivitätsfortschritt in der privatwirtschaftlichen Lohnentwicklung berücksichtigt. Dagegen wird aufgrund der bekannten Meßproblematik der Index der öffentlichen Tätigkeit in der Regel ohne derartige Korrekturen ermittelt. Folglich fällt der Preisindex für öffentliche Tätigkeit (Zähler der Staatsquote) tendenziell höher aus als der Preisindex für das BIP (Nenner), wenn das Produktivitätswachstum die Preisentwicklung im privaten Sektor mit beeinflußt.

Der „relative Preiseffekt“ könnte darauf hinweisen, daß das Wachstum der Staatsquote nur scheinbar ist und in einem statistischen Meßfehler begründet liegt. ${ }^{236}$ Diese Interpretation findet jedoch wenig Anhänger in der Literatur, und eine Regierung, die eine Senkung der nominalen Ausgabenquote anstrebt, wird ihr auch nicht viel Beachtung schenken. Zudem bedeutet ein relativer Preiseffekt unabhängig von seiner Ursache, daß der nominale Finanzierungsbedarf ebenfalls steigen muß. Deswegen wird diese Interpretation hier nicht weiter erörtert. Eine bekannte Erklärung für den „relativen Preiseffekt“ bietet Baumol (1967). Staatliche Aktivitäten umfassen viele arbeitsintensive Dienstleistungen, wie z. B. die innere Sicherheit, die Gesundheitsvorsorge und das Erziehungswesen. Im Unterschied zur kapitalintensiven Güterproduktion im Privatsektor, wo technologischer Fortschritt groBe Produktivitätssteigerungen ermöglicht, gibt es laut Baumols These wenig Potential für die Substitution von Arbeit durch Kapital im öffentlichen Sektor. Wenn die Produktivität im öffentlichen Sektor tatsächlich niedriger ausfällt als im privaten Sektor, sich aber die Entlohnung der Arbeitskräfte in beiden Sektoren gleich-

234 Siehe Insbesondere Heller (1981), sowie Peacock (1983).

${ }^{235}$ Für eine Definition, siehe Oxley (1990), S. 5.

${ }^{236}$ Dieser Ansicht ist z. B. Heller (1981), S. 64. 
mäßig entwickelt, dann werden sich im Zeitverlauf die öffentlichen Leistungen relativ verteuern. Daraus ließe sich das Wachstum der Staatsausgaben für öffentlich bereitgestellte Güter und Dienstleistungen erklären.

Nun ist es aber erstens durchaus nicht offensichtlich, daß technologischer Fortschritt im öffentlichen Sektor weniger möglich sei als im privaten. Die neue Kommunikations- und Informationstechnologie bietet dem öffentlichen wie auch dem privaten Sektor gleichermaßen produktivitätssteigerndes Potential. ${ }^{237}$ Zweitens setzt die These voraus, daß sich die Löhne bzw. ihre Zuwachsraten, in beiden Sektoren parallel entwickeln. Der öffentliche Sektor sieht sich danach gezwungen, den im privaten Sektor bezahlten Löhnen zu entsprechen, um die Abwanderung seiner Arbeitskräfte zu verhindern [Baumol (1967), S. 417]. Ob dieser Zwang besteht, hängt von den institutionellen Gegebenheiten, insbesondere vom Lohnbildungsproze $\beta$ auf dem Arbeitsmarkt ab. A priori ist jedoch auf einem Wettbewerbsmarkt nicht zu erwarten, daß die öffentlichen Bediensteten trotz unterstellter geringerer Produktivität die gleiche Entlohnung wie im Privatsektor durchsetzen können. Geschähe dies, dann läge die steigende Staatsquote weniger in den Bedingungen der staatlichen Güterbereitstellung, als in der Lohnentwicklung im Staatssektor begründet, welche die Produktivitätsfortschritte überschreitet. Dann wären die Arbeitsbedingungen und die Lohnverhandlungsmacht im öffentlichen Dienst zu untersuchen.

Eine relative Verteuerung der öffentlichen Produktion könnte jedoch mit institutionellen Ineffizienzen, insbesondere mit mangelndem Wettbewerb oder falschen Produktivitätsanreizen bei den staatlichen Anbietern oder Bürokraten zusammenhängen. ${ }^{238}$ Diese könnten ineffizient arbeiten, aber trotzdem produktivitätsüberschreitende Lohnforderungen durchzusetzen und möglicherweise als Interessengruppe auftreten. Dieser Ansatz wird in den Theorien der Bürokratie behandelt.

\section{b) Der Einfluß der Bürokratie}

Im Sinne der tatsächlichen Bereitstellung sind die Anbieter von staatlichen Leistungen nicht die Politiker, sondern die Staatsangestellten oder die Bürokraten, wie sie gewöhnlich in der Literatur genannt werden. Bürokratietheorien unterstellen, $\mathrm{da} \beta$ Bürokraten, wie andere Wirtschaftssubjekte auch, Nutzenmaximierer sind und möglicherweise ein Interesse an einer erhöhten Staatstätigkeit haben, welche wenn sie dieses umsetzen können - zu einem Anstieg der Staatsquote führen kann. Die bekannteste Begründung dieser Hypothese lieferte Niskanen (1971). Seine Bü-

\footnotetext{
${ }^{237}$ Burton (1985), S. 65

${ }^{238}$ Hierzu z. B. Peacock (1979).
} 
rokratietheorie übte auch einen großen Einfluß auf die Regierung Thatcher und deren Bemühungen zu Ausgabensenkungen aus. ${ }^{239}$ Nach Niskanen (1971) liegt dem Einfluß von Bürokraten auf die Höhe der Staatsausgaben ein Principle-AgentProblem infolge asymmetrischer Information zugrunde. Wegen der Mess- und Bewertungsproblematik bei öffentlichen Leistungen kennen die Volksvertreter bzw. die Wähler, wenn von anderen Transmissionsverzerrungen abstrahiert wird, die wahren Kosten des bürokratischen Angebots nicht. Ihnen wird von den Bürokraten ein bestimmtes, unteilbares Leistungsvolumen für ein bestimmtes Budget angeboten, welches sie entweder annehmen oder ablehnen können. Neben diesem institutionellen Merkmal der Budgetverhandlungen zwischen Bürokraten und Politikern nimmt Niskanen (1971) ferner an, daß Gehalt, Prestige, Macht und Aufstiegsmöglichkeiten den Bürokraten Nutzen stiften und daß diese Dinge direkt von der Budgethöhe abhängen. Folglich streben Bürokraten ein möglichst hohes Budget an. Zudem wird angenommen, daß ein von den Wählern akzeptiertes Kosten-NutzenVerhältnis die einzige Beschränkung des bürokratischen Handelns darstellt. Weil nicht über einzelne Preise und Leistungen, sondern nur über ein gesamtes Budgetpaket verhandelt wird, akzeptieren die Wähler ein überhöhtes Angebot lieber als gar keins. Das Ergebnis ist eine Ausdehnung des staatlichen Leistungsvolumens über die optimale Menge hinaus. ${ }^{240}$

Niskanens (1971) Annahmen über die bürokratischen Nutzenquellen sowie die mangelnden Beschränkungen ihrer Handlungsfreiheit sind ausführlich diskutiert worden. ${ }^{241}$ Die am häufigsten geäußerte Kritik daran bezieht sich auf das unterstellte Maximierungsverhalten der Bürokraten. Statt dessen wird postuliert, daß die Bürokraten nicht den Budgetumfang sondern ihre Freizeit und die Bequemlichkeit ihrer Arbeit zu erhöhen suchen. Anstelle eines größeren Outputs und entsprechender Budgeterhöhung, bieten die auf Freizeit und Bequemlichkeit bedachten Bürokraten einen gegebenen Output zu überhöhen Kosten an. Entsprechend dieser Auslegung bürokratischen Verhaltens wird die von Niskanen (1971) abgeleitete allokative Ineffizienz durch die sogenannte X-Ineffizienz ersetzt. ${ }^{242}$

Eine zweite wichtige Kritik an Niskanen (1971) bezieht sich auf den statischen Charakter seiner Überlegungen. Er zeigt zwar, warum das Niveau der Staatsausgaben zu hoch ausfallen kann. Ein Anstieg im Sinne der Wagner'schen These läßt sich jedoch daraus nicht ableiten. ${ }^{243}$ Wenn der Einfluß von Bürokraten als Ursache

${ }^{239}$ Niskanen (1973) zählte zur Pflichtlektüre der Bürokraten im Finanzministerium unter Keith Joseph, Cullis und Jones (1993), S. 86.

${ }^{240}$ Eine anschauliche Darstellung bieten Cullis und Jones (1993), S. 87ff.

241 Siehe z. B. Müller (1989), S. 254ff., Musgrave (1985), S. 24ff., Becker (1990), S. $50 \mathrm{ff}$. und die dort zitierte Literatur.

242 Leibenstein (1966).

${ }^{243}$ Pommerehme und Kirchgässner (1988), S. 236. 
für das Wagner'sche Gesetz interpretiert wird, dann muß im Zeitverlauf ihr Einfluß bzw. ihre Macht zunehmen. Dies könnte sich entweder in einer Beschäftigungszunahme im öffentlichen Dienst oder qualitativen Veränderungen, wie z. B. größere Intransparenzen im Principle-Agent Verhältnis äußern. Die zwei bekanntesten Versuche, Niskanens Gedanken zu dynamisieren, greifen diese Möglichkeiten auf: Eine ergänzende Hypothese über das Wahlverhalten von Bürokraten als Interessengruppe [Tullock (1974)] fußt auf einer zunehmenden öffentlichen Beschäftigung; ${ }^{244}$ erschwerte Kontrollen und steigende Überwachungskosten bei großen Bürokratien [Mueller (1989)] unterstellt größeren Bürokratien relativ stärkere Einflußmöglichkeiten. ${ }^{245}$

Verursacht die Tätigkeit von Bürokraten ein überhöhtes Angebot an Staatsleistungen, dann läßt sich dies entweder in zunehmender Beschäftigung im staatlichen Sektor oder Anzeichen von zunehmender X-Ineffizienz ablesen. Recherchen in 49 vergleichenden Studien über vier Länder bis 1982 ergab, daß das öffentliche Angebot nur in 3 Fällen effizienter und nur in 9 gleich effizient wie das private ermittelt wurde. ${ }^{246}$ Eine britische Untersuchung über drei Aktivitäten, ${ }^{247}$ wo private und staatliche Anbieter miteinander konkurrieren, stellte bei den Staatsbetrieben zwischen 10 und $20 \%$ höhere Kosten fest.

\section{c) Die Entscheidungsstrukturen und -praktiken der Regierung}

Unter der Kategorie Entscheidungsstrukturen und -praktiken der Regierung lassen sich drei wichtige und sehr breite Aspekte unterscheiden. ${ }^{248}$ Erstens gibt es verfassungsmäßige Strukturmerkmale, welche polit-ökonomische Entscheidungsorgane beeinflussen und Auswirkungen auf die Staatsausgaben haben können. Beispiele sind der Zentralisierungsgrad von polit-ökonomischen Entscheidungsgremien, das Wahlsystem oder die Haushaltsregeln und -verfahren. ${ }^{249}$ Zweitens werden Ausga-

244 Bürokraten berücksichtigen ihre Interessen auch bei ihrem Wahlverhalten. Somit treten sie als Interessengruppe auf, deren Einfluß sich verstärkend ausweitet mit zunehmender Größe.

245 Größere Bürokratien sind schwieriger zu überwachen und bieten folglich zunehmende Möglichkeiten, Preise und/oder Output zu manipulieren.

246 Borcherding, Pommerehe und Schneider (1982), S.130-3.

${ }^{247}$ Luft- und Kanalferryverkehr sowie Gas- und Elektrizitätswerke, siehe hierzu Pryke (1982).

248 Überblicke bieten Frey und Schneider (1981) und Paldam (1981).

${ }^{249} \mathrm{Vgl}$. Hamlin (1993). 
benentscheide vom politischen Konjunkturzyklus beeinflußt. ${ }^{250}$ Drittens wirkt sich die Gestaltung des Entscheidungsprozeßes auf die Höhe der Ausgaben aus. ${ }^{251}$

Es ist offensichtlich, daß die Bedeutung dieser Aspekte stark von den institutionellen Merkmalen der jeweiligen Länder abhängt. Für den Fall des Vereinigten Königreichs erscheinen Ansätze, welche sich auf politische Fragmentierung in der Regierung oder abwechselnde Machtverhältnisse stützen, uninteressant, weil derartige politische Gegebenheiten auf das Vereinigte Königreich zwischen 1979 und 1994 nicht zutreffen. Dagegen verdienen der seit 1979 gestiegene Zentralisierungsgrad von Ausgabenentscheidungen, die Vorgabe von Budgetregeln und Ausgabenzielen, oder Reformen bei ministeriellen Entscheidungsprozeduren größere Aufmerksamkeit. Sie wurden teilweise bereits untersucht. ${ }^{252} \mathrm{Um}$ diese Theorien zu behandeln, wäre als erstes eine Darstellung der institutionellen Gegebenheiten im Vereinigten Königreich erforderlich. Zweitens setzen diese Ansätze ohnehin andere Ursachen der steigenden Staatstätigkeit voraus, welche im Zeitverlauf Schwächen in einem in der Regel lang unveränderten konstitutionellen Rahmenwerk offenlegen. Institutionelle Schwächen verstärken beispielsweise den Einfluß von Bürokraten, Interessengruppen oder andere Intransparenzen im Wählerprozeß. Deswegen sind diese Theorien als Ergänzungen zu den oben diskutierten Ansätzen zu sehen. Um den Umfang der Diskussion einzugrenzen, wird deswegen in dieser Abhandlung auf die weitere Verfolgung dieser Ansätze verzichtet.

\section{4) Schlußfolgerungen für die Strategien zur Ausgabensenkungen}

Ausgangspunkt der Überlegungen war die Annahme, daß das Niveau der Staatsausgaben von der Nachfrage, d. h. von den marginalen Entscheidungen der Wirtschaftssubjekte beeinflußt wird. Neben dem Einfluß von ökonomischen Größen wie Einkommen und Konsumentenpräferenzen auf diese Entscheidungen, werden sie auch von politischen Faktoren beeinflußt, wie z. B. der Ausgestaltung des Wahlrechts, der Einkommmensverteilung und der Macht von Interessengruppen. Offenbart, wie bei den traditionellen Ansätzen unterstellt, der politische Prozeß die Wählerpräferenzen, dann sollte die Höhe der Staatsausgaben den Wählerwünschen entsprechen. Eine Regierung dürfte dann kaum hoffen, die Staatsausgaben dauerhaft unter das gewünschte Niveau zu senken. ${ }^{253}$ Allerdings zeigen neuere Arbeiten,

${ }^{250}$ Vgl. Nordhaus (1989).

251 Vgl. Cusack (1988).

252 Siehe z. B. Peacock (1983), Burton (1985)

253 Selbst die der Reduktion der Staatsausgaben verpflichtete britische Konservative Regierung war seit 1979 wiederholt gezwungen, das Angebot des staatlichen Gesundheitssystems (NHS) aufrecht zu erhalten, weil dies die Wählermehrheit wünschte. Hierzu ausführlicher im 5 . Kapitel. 
wie die Präferenzoffenbarung des Wahlsystems durch das Verhalten von bestimmten Bevölkerungsgruppen bzw. Interessengruppen verzerrt werden kann. Aus der obigen Diskussion deuten sich für eine Regierung drei Möglichkeiten an, Einfluß auf die Nachfrage auszuüben.

Erstens ist zu erwarten, daß die Wähler einer Substitution staatlich bereitgestellter Güter zugunsten eines privaten Angebots zustimmen, wenn jene Kosten- und Preisentwicklung fortbesteht, welche der wie auch immer begründete ,relative Preiseffekt" beschreibt. Eine Preisinelastizität der Nachfrage erscheint auf Dauer nur für rein öffentliche Güter plausibel, welche nicht durch private Bereitstellung substituiert werden können. Deshalb dürfte bei steigenden Preisen für die öffentliche Bereitstellung die Akzeptanz für ein privatwirtschaftliches Ersatzangebot zunehmen. Die Regierung kann dann einen Teil der staatlichen Aktivitäten der privaten Versorgung übergeben. Die Möglichkeiten sind vielfältig und erstrecken sich von der Einführung von Wettbewerb und anderen marktorientierten Mechanismen im staatlichen Angebot, über Managementsysteme bis zur Auftragsvergabe an den privaten Sektor. ${ }^{254}$

Zweitens zeigt das Verteilungsmodell von Meltzer und Richard (1981), wie eine ungleiche interpersonelle Verteilung zu steigenden Staatsausgaben führen kann. Die offenkundige „Lösung“ für dieses Problem wäre es, den ärmeren Gesellschaftsmitgliedern das Wahlrecht zu entziehen oder einzuschränken. ${ }^{255}$ Das Verteilungsmodell legt zwei andere Lösungen nahe. Zum einen könnte die Dezentralisierung der Ausgaben durch die Ausbildung von stärkeren föderalistischen Strukturen alternative staatliche Angebotsquellen in konkurrierenden Gebieten schaffen. Ziel einer derartigen Politik wäre es, die Macht des nationalen Medianwählers zu reduzieren. Zum anderen könnte die Regierung durch nicht ausgabengelenkte verteilungspolitische Maßnahmen versuchen, die Ungleichheit der interpersonellen Verteilung zu senken. Beispiele dafür sind die Festlegung von Mindestlöhnen oder Quoten für die Beschäftigung von benachteiligten Minderheiten (z. B. Behinderten). Derartige Maßnahmen versuchen, die Faktorentgelte anzugleichen. Weiter können Steuern auf Erbschaften und Schenkungen, sowie der kostenlose Zugang zur Bildung den Besitz von physikalischem und humanem Kapital gleichmäßiger streuen. ${ }^{256}$

Drittens zeigte die Diskussion über Interessengruppen, daß das staatliche Angebot sich nicht unbedingt an die Wählerwünsche insgesamt anpaßt, sondern möglicher-

\footnotetext{
254 Ausführlicheres zur britischen Politik im 4. Kapitel, Abschnitt III.3.b.

255 Die Einführung der sogenannten „Poll-tax“ (übersetzt Wahl-Steuer) im Jahre 1990 (vgl. Fußnote 184) hatte eine derartige Nebenwirkung. Erhebungsgrundlage der Steuer war das Wahlregister. Folglich ließ sich durch Abmeldung vom Wahlregister, die Steuer umgehen.
}

${ }^{256}$ Cullis und Jones (1992), S. 233. 
weise bestimmte Interessengruppen bevorzugt. Politische Dezentralisierung kann hier auch sinnvoll sein, wenn sie die Macht von Interessengruppen auf nationaler Ebene einschränkt. Der relative Preiseffekt als Ursache steigender Staatsausgaben deutet entweder auf übermäßige Lohnabschlüsse und/oder sogenannte XIneffizienzen und somit auf die Rolle von Staatsbediensteten als Interessengruppe. Um den Einfluß von dieser Gruppe einzuschränken, kann der Staat $z$. B. versuchen, durch Dienst- und Besoldungsreformen die Lohn- und Gehaltskosten zu senken und/oder die Produktivität von Staatsangestelltern zu erhöhen. Existiert hier ein Potential für effizienzsteigende Maßnahmen, dürfte dieses ebenfalls Akzeptanz bei der gesamten Wählerschaft finden.

\section{Strategie IV: Die Veräußerung von öffentlichem Vermögen}

Dieser Abschnitt untersucht die Möglichkeiten, durch Veräußerung oder Privatisierung ${ }^{257}$ von öffentlichem Vermögen das Budget zu entlasten und einen Beitrag zum Schuldenabbau zu leisten. Im Abschnitt I.2.c wurde gezeigt, daß Bond- und Aktivaverkäufe aus finanzpolitischer Sicht weitgehend äquivalent sind, wenn von perfekter Voraussicht, vollkommenen Kapitalmärkten, sowie identischer Produktionseffizienz im öffentlichen und privaten Sektor ausgegangen wird.

Die Aufhebung obiger restriktiver Bedingungen kann dieses Ergebnis modifizieren, wenn der Staat und der Privatsektor aufgrund unterschiedlicher Diskontierung nicht zu dem gleichen Gegenwartswert des Vermögens gelangen. Beispielsweise können erstens imperfekte Voraussicht des Privatsektors über die zukünftigen Nettoerträge, sowie eine höhere Risikoaversion des privaten verglichen mit dem öffentlichen Sektor, ${ }^{258}$ dazu führen, daß der Privatsektor eine höhere Diskontierung für den Vermögenserwerb verlangt. Zweitens, wenn der Staat den Diskontfaktor des privaten Sektors nicht kennt, wird er bei seinem Verkaufsangebot den Reservationspreis des Privatsektors möglicherweise unterschätzen. Diese Auswirkungen sind besonders wahrscheinlich, wenn wie in Großbritannien der Staat zwecks einer breiteren Vermögensverteilung kleine, erstmalige Käufer ansprechen möchte, denen Aktienbesitz noch nicht vertraut ist. Eventuelle Verkaufsverluste aufgrund Unterbewertungen werden nicht durch Verkaufsgewinne aufgrund Überbewertun-

${ }^{257}$ Der Begriff „Privatisierung“ im weiteren Sinne wird verwendet, um verschiedene Änderungen in der Beziehung zwischen öffentlichem und privaten Sektor zu bezeichnen, siehe z. B. Blankart (1985). Hier wird er in der engen Abgrenzung verstanden, nämlich als Besitztransfer, vor allem von öffentlichen Unternehmen, vom Staat, an die privaten Haushalte.

258 Die öffentliche Hand kann ihr Risiko breiter streuen als private Individuen, Arrow und Lind (1970). 
gen in anderen Fällen kompensiert, weil für überbewertetes Vermögen kein Kauf zustande kommt. Sollten aus diesen Gründen die erzielten Privatisierungserlöse den Gegenwartswert des Unternehmens nicht erreichen, dann kann die Privatisierung zu einer Netto-Budgetbelastung in zukünftigen Perioden werden. ${ }^{259}$ Wäre dies der Fall, dann ließen sich unter längerfristigen budgetpolitischen Gesichtspunkten Vorbehalte gegenüber einer Privatisierungspolitik während einer Phase der Schuldenkonsolidierung erheben.

Dagegen läßt sich einwenden, daß die Privatisierung eines öffentlichen Unternehmens zu Effizienzverbesserungen und vor allem zu Gewinnerhöhungen führt. Dies ist eine häufige Begründung für die Privatisierung. ${ }^{260}$ Davon profitiert auch der Staat entweder bereits beim Verkaufspreis oder in späteren Perioden aufgrund höherer Steuereinnahmen. Potentielle negative Fiskalwirkungen werden überkompensiert, und die Folge in beiden Fällen ist eine Budgetentlastung und ceteribus paribus ein Verschuldungsabbau. Nach dieser Auffassung ist die Privatisierung eine geeignete Strategie zur Schuldenkonsolidierung.

Neben diesen Überlegungen hängt die Vorteilhaftigkeit von Privatisierungseinnahmen zwecks Schuldenkonsolidierung von den politischen Zielen der Regierung bei der Privatisierungspolitik ab. Der Verkauf von Staatsaktiva zwecks Einnahmebeschaffung wurde bereits von Adam Smith (1776) propagiert. ${ }^{261}$ Darin liegt jedoch die Gefahr, daß erstens die Privatisierung den Schein einer budgetpolitischen Konsolidierung erweckt und den Druck zu anderen Reformen mindert. Zweitens kann die politische Zweckmäßigkeit kurzfristiger Einnahmen den Staat zu unterbewerteten Verkäufen bewegen. ${ }^{262}$ Yarrow (1986) dokumentiert teilweise massive Aktienpreiserhöhungen nach der Handelsfreigabe von privatisierten, britischen Staatsbetrieben, welche auf Unterbewertungen hindeuten.

Die flankierende Regulierung und wettbewerbspolitische Maßnahmen bei einer Privatisierung sind ebenfalls wichtig für die langfristigen budgetpolitischen Auswirkungen, weil diese die Effizienz des privatisierten Betriebs beeinflußen. ${ }^{263} \mathrm{Da}-$ bei sind es weniger die Eigentumsverhältnisse per se, sondern Wettbewerbsbedingungen, welche die notwendige Voraussetzung für erhöhte Effizienz darstellen. ${ }^{264}$ Führt die Privatisierung zu Effizienzverbesserungen ohne ein für den Staat kost-

\footnotetext{
259 Ein Verkauf von staatlichen Vermögen an das Ausland bedeutet in diesem Fall einen realen Vermögensverlust für das Inland, Yarrow (1986), S. 359.

${ }^{260}$ Mansoor (1993), S. 354

${ }^{261}$ Siehe hierzu Yarrow (1986), S. 324.

262 Bös (1989), S. 234.

263 Yarrow (1986), S. $361 \mathrm{f}$.

${ }^{264}$ Djaroueh (1994), S. 216 und die dort zitierte Literatur. 
spieliges Marktversagen zu verursachen, dann sind langfristig Einnahmeverbesserungen zu erwarten. Dies kann der Fall sein, wenn die betroffenen Industrien einem Wettbewerbsmarkt ausgesetzt werden. Wenn jedoch unangetastete staatliche Monopolbetriebe veräußert werden - ohne begleitende Regulierung oder Förderung des Wettbewerbs - beruht der finanzielle Erfolg der privatisierten Betriebe auf der effizienten Ausbeutung ihrer Marktmacht, und nicht auf effizienter Produktion unter Wettbewerbsbedingungen. Dies führt zwar kurzfristig zu höheren Erlösen aus der Privatisierung, weil höhere zukünftige Gewinne erwartet werden. Langfristig entstehen jedoch volkswirtschaftliche Kosten in Form von Effizienzverlusten, welche sich negativ auf die Budgeteinnahmen wirken. ${ }^{265}$ Insbesondere wenn zwecks Einnahmenbeschaffung die Preise von Produkten aus Staatsbetrieben hoch, jedoch zwecks Ausgabenersparnis die Investitionen niedrig gehalten werden, wie dies seit 1979 im Vereinigten Königreich der Fall ist, dann wird die politische Versuchung sehr groß, das Preisniveau durch wettbewerbspolitische Schutzmaßnahmen hoch zu halten, um den Erlös bei einer Privatisierung zu steigern. Die Effizienz der betroffenen Betriebe und damit auch der zukünftige budgetpolitische Beitrag wird in diesem Fall jedoch niedriger. ${ }^{266}$

Schließlich hat das Vertrauen und die Unterstützung der Öffentlichkeit einen Einflu $\beta$ auf den Preisbildungsproze $\beta$. Die Angst einer Wieder-Nationalisierung oder der Besteuerung von „windfall“ Gewinnen infolge eines Regierungswechsels wirkt sich negativ auf den budgetpolitischen Beitrag einer Privatisierung aus.

Zweifelslos erbringen die Mittel aus einer Privatisierung eine kurzfristige Budgetentlastung. Die Diskussion zeigte jedoch, da $\beta$ ihre langfristigen Wirkungen infolge Einflüsse vieler Rahmenbedingungen auch negativ ausfallen kann. Noch ist unklar, welche Effekte dominieren und wie die Privatisierung budgetpolitisch längerfristig zu beurteilen ist. ${ }^{267}$ Es besteht auf jeden Fall die Gefahr, aus den kurzfristigen und einmaligen Einnahmen eine scheinbare Konsolidierung abzuleiten.

\section{Zusammenfassung des zweiten Kapitels}

In diesem Kapitel werden die verschiedenen Möglichkeiten zur Senkung der Staatsverschuldung identifiziert und diskutiert. Inflation wirkt sich für den Staat in

\footnotetext{
${ }^{265}$ Kay und Thompson (1986), S. 28ff. In diesem Sinne beurteilt der „Economist“ vom 13.04.96 z. B. die Wettbewerbsentwicklung der privatisierten britischen Elektrizitätsindustrie, S. $31 \mathrm{f}$.

${ }^{266}$ Hierzu Eltis (1983), S. 100ff.

${ }^{267}$ Eine neue Untersuchung kommt sogar zu einem skeptischen Urteil der langfristigen Budgetwirkungen, Djaroueh (1994), S. 204ff. 
verschiedener Weise günstig auf die Budgetfinanzierung aus. Wenn er sich aus der Geldschöpfung finanziert, kann dies nach quantitätstheoretischen Zusammenhängen einen Preisanstieg auslösen. Die Geldschöpfung ist damit nicht nur eine griffige kurzfristige Finanzierungsquelle, sondern führt längerfristig über die dadurch ausgelöste Inflation zu einer realen Schuldentilgung. Selbst wenn der Staat für seine Finanzierung nicht zur Notenpresse greift, kann er trotzdem versucht sein, Inflation zu dulden, weil die resultierende Schuldentilgung seine Budgetbeschränkung lockert.

Eine mikroökonomische Betrachtung anhand einer erweiterten Lafferkurve, sowie ein Vergleich der konkurrienden Steuersätze im europäischen Ausland zeigt den Spielraum für Steuereinnahmen zur Erhöhung des Aufkommens auf. Die politische Unbeliebtheit von Steuererhöhungen macht diese Option der Mittelbeschaffung jedoch unattraktiv. So haben beispielsweise kollektive Bewegungen des Steuerwiderstands im Vereinigten Königreich die Erhebung von bestimmten Steuern verhindert.

Ausgabenkürzungen werden mit Bezugnahme auf die Theorien der wachsenden Staatstätigkeit erörtert. Daraus wird deutlich, daß generell ein Aufwärtsdruck auf die Ausgaben besteht und deshalb für eine Senkung der Ausgaben überkompensiert werden müßte. Soziale, demographische und technologische Kräfte üben vor allem einen Druck auf diejenigen Ausgaben aus, welche Umverteilungscharakter haben. Dies betrifft primär die Bereiche Erziehung und Gesundheit, aber auch die Transferzahlungen. $\mathrm{Zu}$ diesen gesellschaftlichen Veränderungen kommt die von Meltzer und Richard (1981) formulierte These hinzu, wonach eine zunehmende Ungleichmäßigkeit der Einkommensverteilung die Nachfrage nach staatlicher Verteilungspolitik erhöht. Auf der Angebotsseite wird gezeigt, wie verschiedene Ineffizienzen entstehen können, welche ebenfalls einen Aufwärtsdruck auf die Ausgaben ausüben. Die Anwendung verschiedener marktorientierter Mechanismen, einschließlich Maßnahmen zur Reform der Dienst- und Besoldungsbedingungen der Staatsangestellten können die Effizienz und Effektivität der Staatstätigkeit erhöhen und dadurch Ersparnisse erzielen.

Privatisierung bringt kurzfristige Einnahmen ein. Ihre langfristige Wirkung auf die staatliche Budgetbeschränkung ist jedoch ungewiß. Entscheidend ist die Werteinschätzung von Verkäufern und Käufern. Wenn ein Verkaufsabschluß zustande kommt, welcher das Vermögen unterbewertet, dann übersteigen die zukünftigen Erträge den Gegenwartswert des veräußerten Vermögens. Eine Privatisierung unter diesen Bedingungen bedeutet deshalb langfristig eine Netto-Budgetbelastung. 
Judith Safford - 978-3-631-75199-2

Downloaded from PubFactory at 01/11/2019 07:12:45AM

via free access 


\title{
Drittes Kapitel
}

\section{DIE SCHULDENPOLITIK DER KONSERVATIVEN REGIERUNG}

Nach der theoretischen Darstellung und Diskussion der möglichen Strategien zur Senkung der Staatsverschuldung im vorigen Kapitel, geht es im folgenden Kapitel um die konkrete Schuldenpolitik der Konservativen Regierung im Vereinigten Königreich. Diese wird besser verständlich vor dem Hintergrund der britischen Wirtschaftsgeschichte der Nachkriegszeit und der wirtschaftspolitischen Konzeption der damaligen Regierungen. Nach einem kurzen Überblick über den historischen Verlauf der britischen Staatsverschuldung werden deshalb die wesentlichen Züge der britischen Wirtschaftsentwicklung und -politik bis zur Machtübernahme der Konservativen Regierung 1979 kurz umrissen. Danach folgt eine Darstellung und Diskussion der Strategien und Ziele der Konservativen Schuldenpolitik.

\section{Historischer Abriß der Staatsverschuldung im Vereinigten Königreich}

\begin{abstract}
Abbildung 9 zeigt die historische Entwicklung der Verschuldung des britischen Zentralstaats im Verhältnis zum Bruttosozialprodukt seit 1700 und somit die Schwankungen der Schuldenquote über fast dreihundert Jahre.
\end{abstract}

Auffallend ist zunächst, daß eine hohe Verschuldung keine Neuigkeit ist. Bereits im frühen 18 . Jh. überschritt die Verschuldungsquote $60 \%$ und erreichte damit ein Niveau, das entsprechend den 1991 vereinbarten Maastrichter-Bedingungen für die Aufnahme in die Europäische Währungsunion als Obergrenze gilt. Im Jahre 1764 kurz vor der Veröffentlichung von Adam Smiths „Wealth of Nations“ - erreichte die Schuldenquote sogar $130 \% .^{268}$

268 Die Zahlen gehen auf die Arbeit von Barro (1987) zurück. Die Schätzungen sind vor allem vor 1830 mit großer Unsicherheit behaftet. Grundsätzlich waren die damaligen Erfassungs- und Bewertungsprobleme für Datensammler und Statistiker noch größer als heute. Die Zahlen zu den Staatsfinanzen wurden jedoch relativ gut dokumentiert. Dagegen geht die Statistik zum nominalen BSP nur bis 1830 zurück. Frühere Werte wurden aus einem Deflator für den Großhändler und einer Schätzung des Trendwachstums für das reale BSP konstruiert. Eine ausführliche Beschrei- 


\section{Abbildung 9: Die Staatsverschuldung im Vereinigten Königreich seit 1700}

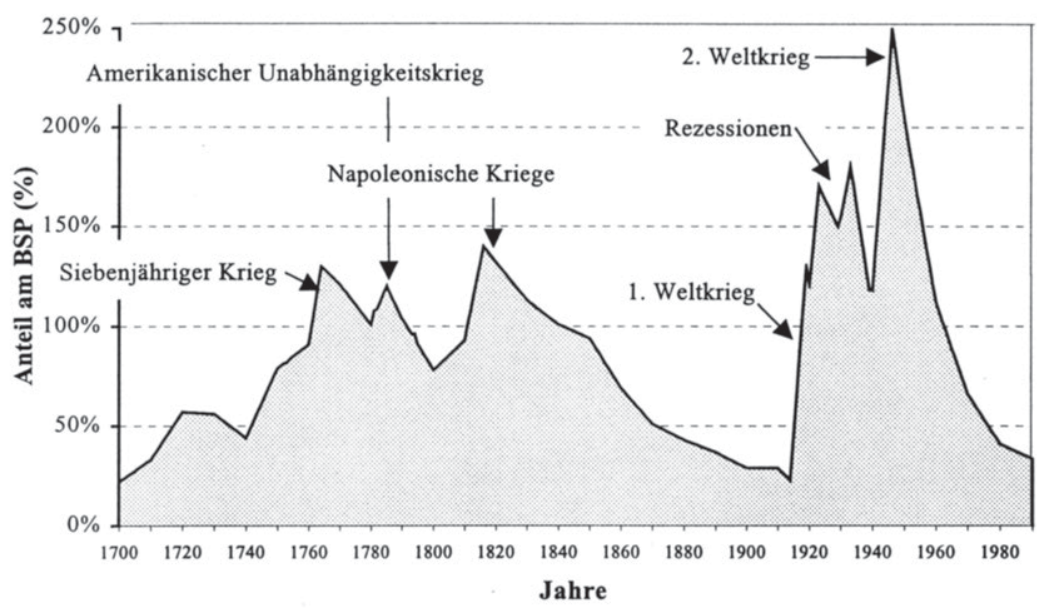

Quellen: Barro (1987), Barro und Grilli (1994)

In den folgenden Jahrzehnten wurden die sogenannten „Klassischen Prinzipien“ der Staatsverschuldung u. a. von Hume (1741), Smith (1776) und Ricardo (1820) entwickelt. Dieser Zeitraum gilt als eine Epoche der fiskalischen Vernunft und „Austerity“. ${ }^{269}$ Die Graphik macht deutlich, daß sich dies trotz des damals aktuellen Interesses der Ökonomie an der Staatsverschuldung nicht in schuldenpolitischen Konsequenzen niederschlug. Im Gegenteil: Die durchschnittliche Schuldenquote lag zwischen 1760 und 1860 bei circa $100 \% .{ }^{270}$

Die Zeitreihe verdeutlicht ebenfalls den bekannten Zusammenhang, daß die Staatsverschuldung in Kriegs- oder Rezessionszeiten rapide ansteigt. In Zeiten des Friedens und des Wirtschaftswachstums nimmt die Verschuldungsquote wieder ab. ${ }^{271}$ Besonders deutlich sind die Maxima im Zusammenhang mit hohen Kriegsausgaben: für den Krieg der Spanischen Nachfolge (1702-13), den Siebenjährigen Krieg

bung der empirischen Vorgehensweise findet sich bei Barro (1987) sowie seiner Originalquelle Mitchell und Deane (1962).

269 So beschreiben z. B. Buchanan und Wagner (1977) in „Democracy in Deficit. The Political Legacy of Lord Keynes“ die Prinzipien und Praxis der Klassischen Verschuldungspolitik vor 1946 im Kapitel 2, „The Old-Time Fiscal Religion“.

${ }^{270}$ Der Mittelwert der Schuldenquote für den Zeitraum 1760-1860 beträgt 106\%.

271 Bereits die Klassischen Ökonomen erkannten die zentrale Bedeutung von Großbritanniens fast ununterbrochener Kriegführung für die Staatsfinanzen, siehe z. B. Smith (1776), S. 412. Auf die Schwankungen zwischen Kriegs- und Friedenszeiten macht Pigou (1928) Kap. VI aufmerksam. Barro (1979) formalisierte diesen Gedanken mit seiner Theorie des „Tax-Smoothing“" 
(1756-1763), den Amerikanischen Unabhängigkeitskrieg (1775-1783), die Kriege mit Frankreich, einschließlich der Napoleonischen Kriege (1793-1815), sowie die zwei Weltkriege. Nach dem Ersten Weltkrieg (1914-1918) stand die Schuldenquote wieder auf 130\%. Nach dem Zweiten Weltkrieg (1939-1945) wurde 1946 der historische Höchststand von $250 \%$ erreicht. Ebenfalls deutlich zu erkennen sind die Auswirkungen der Rezessionen von 1920-23 und 1929-1933. Damals stieg die Schuldenquote von $121 \%$ auf $170 \%$, beziehungsweise von $150 \%$ auf $180 \%$.

Die Schuldenquote ist eine Verhältniszahl, ${ }^{272}$ die sich zusammensetzt aus der absoluten Höhe der Staatsschuld (d. h. die finanziellen (Brutto) Verbindlichkeiten des Staatssektors) im Zähler und einem geeigneten Einkommenskonzept im Nenner. Folglich läßt sich ihre dynamische Entwicklung aus den Bewegungen dieser Bestandteile erklären. Kriegführung führt vor allem zu einer temporären Erhöhung der Staatsausgaben, was den Schuldenstand ansteigen läßt. Wirtschaftliche Rückgänge wirken sich einerseits negativ auf das Einkommen und andererseits positiv auf die Schuldenaufnahme aus.

Die Abbildung 10 fokussiert nun die letzten zwanzig Jahre: Sie zeigt die Entwicklung der Schuldenquote, wie sie von der Bank of England (BoE) seit 1975 ermittelt wird. ${ }^{273}$ Zum Vergleich sind auch die von der OECD (1995) und von Barro (1987) ermittelten Zeitreihen dargestellt.

Die BoE-Zeitreihe (sowie auch diejenige von der OECD) umfaßt neben dem Zentralstaat auch die Verschuldung der Gemeinden ${ }^{274}$ und der staatlichen Unternehmen. Sie ist auf das Bruttoinlandsprodukt bezogen, das im UK etwas kleiner als das BSP ausfällt. Deshalb wäre zu erwarten, daß diese Quoten höher liegen als Barros Zeitreihe. ${ }^{275}$ Dies ist auch bis 1980 der Fall. Danach nähern sich die Quoten an, was auf die Konzentration der Verschuldung auf den Zentralstaat zurückzuführen ist.

272 Streng genommen ist sie keine echte Verhältniszahl, hierzu Francke und Kotz (1994) S. 12f.

${ }^{273}$ Die im Maastrichter Vertrag vereinbarte Schuldenquote liegt geringfügig höher (circa 1-2\%).

274 Die lokalen Gebietskörperschaften im Vereinigten Königreich sind die Districts (ungefähr mit dem deutschen „Kreis“ gleichzusetzen) und die Councils (Gemeinde- und Stadträte), welche das Hauptorgan darstellen. Der Einfachheit halber werden diese kommunalen Gebietskörperschaften zusammen als Gemeinden bezeichnet, obwohl dies nicht genau der deutschen Verwendung des Begriffs entspricht.

275 Die Abweichung zwischen OECD- und BoE-Zahlen liegt an unterschiedlichen Erfassungs- und Bewertungsverfahren zwecks internationaler Vergleichbarkeits. 


\section{Abbildung 10: Die Entwicklung der Staatsverschuldung im Vereinigten Königreich seit 1975}

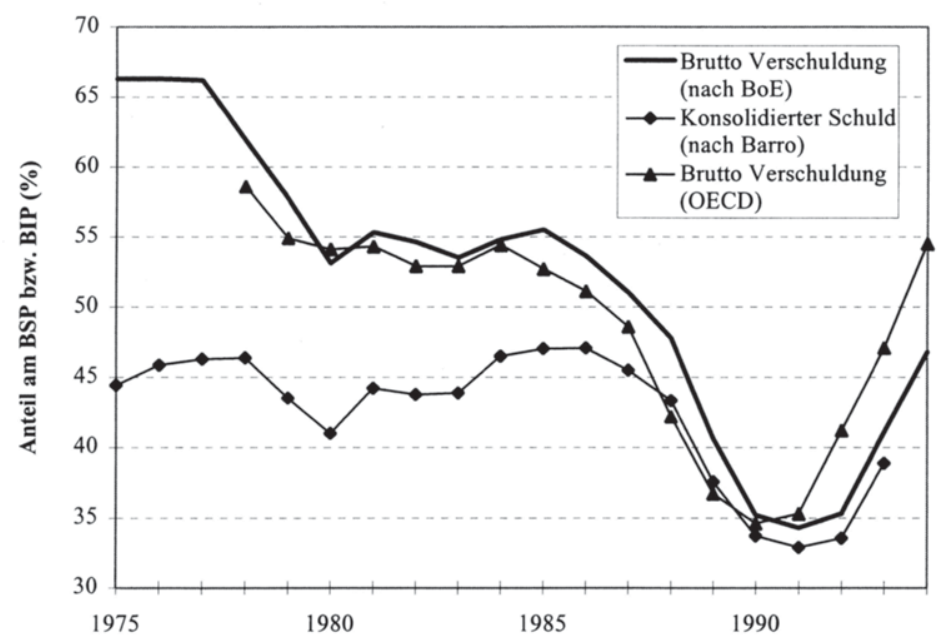

Quellen: BoE, verschiedene Ausgaben, OECD (1995), Barro (1987), Barro und Grilli (1994), eigene Berechnungen

Von 1946 bis 1980 nahm die Schuldenquote regelmäßig ab. 1980 wird dieser Trend unterbrochen. Nach einer anfänglichen Stabilität bis 1985 nimmt die Schuldenquote bis 1990 rapide ab. Nach der Rezession von 1990-91 folgt 1992 erneut ein geringer und nach 1993 ein beträchtlicher Anstieg. Ende März 1995 betrug die britische Staatsverschuldung £342,109 Mrd. und somit 49,4\% des BIP. Gemäß OECD-Prognosen wird die Senkung der 80er Jahre bis 1997 rückgängig gemacht werden. ${ }^{276}$

\section{Aktueller geschichtlicher Hintergrund: Die britische Wirtschaft nach 1945}

Der unablässige Niedergang Großbritanniens von einer großen Weltmacht und einem Wirtschaftskoloß zu einem der ärmeren Länder der Europäischen Union gemessen am Pro-Kopf-Einkommen wurde bereits häufig aufgezeichnet, bedauert,

${ }^{276}$ OECD Wirtschaftsausblick, Dez. 1995, Tab. 34. 
analysiert und zu erklären versucht. ${ }^{277}$ Dabei wird der Beginn dieser Entwicklung bereits vor circa hundert Jahren ausgemacht. In den Jahren nach dem 2. Weltkrieg war die wirtschaftliche Entwicklung Großbritanniens im Vergleich mit den Vorperioden sogar sehr gut und wurde durch hohes Wirtschaftswachstum in Verbindung mit Vollbeschäftigung gekennzeichnet. Die Wachstumsrate der britischen Wirtschaft lag jedoch deutlich niedriger als die anderer großer Industrieländer, so daß bis Anfang der 70er Jahre das Pro-Kopf-Einkommen unter dem OECD Durchschnitt lag. ${ }^{278}$ Die unmittelbare Ursache dieser Entwicklung war die im internationalen Vergleich relativ geringe Produktivitätszunahme in Großbritannien, welche die internationale Wettbewerbsfähigkeit der britischen Industrie stetig schwächte. ${ }^{279}$ Der britische Anteil am Welthandel sank entsprechend: Von circa $25 \%$ Ende der 40 er auf $10 \%$ Anfang der 80 er Jahre. ${ }^{280}$ Die schwache relative Produktivität hatte nicht nur Folgen für den Exportmarkt, sondern betraf auch den Inlandsmarkt. Vor allem in konjunkturellen Boomphasen wurde der Aufschwung weniger durch einen Beschäftigungs- und Produktionsanstieg, als durch erhöhte Importausgaben absorbiert.

Als wirtschaftspolitische Antwort auf diese Entwicklung wurde das britische Pfund zweimal, 1949 und 1967, abgewertet. ${ }^{281}$ Im Juni 1972 wurde nach der Smithsonian Wechselkursvereinbarung das Pfund freigegeben und wertete sich bis 1976 um mehr als $30 \%$ weiter ab. Diese Maßnahmen vermochten jedoch die britische Wettbewerbsfähigkeit nicht dauerhaft wiederherzustellen. Dagegen verursachte die Abwertung Importpreiserhöhungen, welche die Preisstabilität beeinträchtigten. Die britische Inflationsrate lag höher als die anderer westlicher Industriestaaten. Tabelle 2 gibt die wesentlichen britischen Kennzahlen im Vergleich mit der OECD wieder.

${ }^{277}$ Siehe z. B. Kaldor (1966), Bacon und Eltis (1978), Burk und Cairncross (1992), Olson (1982).

${ }^{278}$ Meen (1988), S. xxiv.

${ }^{279}$ Feinstein (1988), S. 4.

${ }^{280}$ Cairncross (1992), S. 286.

2811949 von $\$ 4.02$ auf $\$ 2,80$ und 1967 auf $\$ 2,40$. 
Tabelle 2: Ökonomische Entwicklung des Vereinigten Königreichs und der OECD 1948-79 (Zunahme per annum in \%)

\begin{tabular}{|c|c|c|c|c|c|c|c|c|}
\hline & \multicolumn{2}{|c|}{ BIP } & $\begin{array}{c}\text { Zivile Beschäfti- } \\
\text { gung }\end{array}$ & \multicolumn{2}{c|}{$\begin{array}{c}\text { Arbeits- } \\
\text { produktivität }\end{array}$} & \multicolumn{2}{c|}{ BIP Deflator } \\
\hline & UK & OECD & UK & OECD & UK & OECD & UK & OECD \\
\hline $1948-51$ & 3,1 & $7,0^{\mathrm{a}}$ & 1,0 & - & 2,1 & $(5,9)^{\mathrm{b}}$ & 3,8 & - \\
\hline $1948-56$ & 2,9 & $5,4^{\mathrm{a}}$ & 0,9 & - & 2,0 & $(4,3)^{\mathrm{b}}$ & 4,3 & - \\
\hline $1956-73$ & 3,1 & 4,5 & 0,3 & 1,0 & 2,8 & 3,5 & 4,5 & 4,0 \\
\hline $1973-79$ & 1,5 & 2,6 & 0,2 & 1,1 & 1,2 & 1,5 & 16,0 & 9,0 \\
\hline
\end{tabular}

a) OEEC; b) OEEC pro Einwohner; Quelle: Cairncross (1992), S. 18

Allerdings erklären die stetig sich verteuernden Importe nur einen Teil der Inflationsentwicklung. ${ }^{282}$ Wichtiger waren die Lohnabschlüsse, welche das Produktivitätswachstum laufend überschritten und eine sich fortsetzende Lohn-Preis-Spirale auslösten. Nach verbreiteter Ansicht erklärt sich dies aus den wirtschaftspolitischen Beziehungen zwischen den Sozialpartnern sowie den institutionellen Bedingungen des britischen Arbeitsmarktes. ${ }^{283}$

Leitfaden für die Wirtschaftspolitik nach dem 2. Weltkrieg war das Weißbuch $1944,{ }^{284}$ das die Erkenntnisse von Keynes „General Theory“ (1936) mit der im 2. Weltkrieg erprobten Kooperation zwischen den Sozialpartnern vereinigte. ${ }^{285}$ Nach seiner Forderung war es die Aufgabe der Regierung, anhand Keynesianischer Stabilisierungspolitik für Vollbeschäftigung, eine niedrige Inflationsrate, einen ausgeglichenen Leistungsbilanzsaldo und ein stetiges Wirtschaftswachstum zu sorgen. Darauf baute der gesellschaftliche Konsens sowohl unter Konservativen als auch Labour Regierungen bis 1979. Durch aktive Nachfragesteuerung versuchten die Regierungen der Nachkriegszeit die Beschäftigung und die Produktion anzuregen und somit Wirtschaftswachstum herbeizuführen. Preisstabilität wurde auf mikroökonomischer Ebene durch die Lohn- und Einkommenspolitik angestrebt. Diese

282 Nach Schätzungen der Bank of England (1977), S. 144 waren circa 25\% der Inflation auf die Importpreise und $50 \%$ auf die Lohnentwicklung zurückzuführen.

283 Siehe z. B. Crafts (1986).

284 HMSO (1944).

285 Buiter und Miller (1983), S. $305 \mathrm{f}$. 
setzte die Kooperation der Sozialpartner, insbesondere der Gewerkschaften, implizit voraus.

Die Gewerkschaften befanden sich nach dem Zweiten Weltkrieg in einer sehr starken Machtposition: Die Gesetzgebung der Vorkriegszeit gewährte ihnen weitgehenden Schutz vor Strafverfolgung, und der Mitgliedsanteil unter der Arbeiterschaft sowie das Beschäftigungsniveau auf dem Arbeitsmarkt waren sehr hoch. Bei ihrer Wirtschaftspolitik suchten die Regierungen bis 1979 die Kooperation der Gewerkschaften, weil sie im Namen des Nachkriegskonsens den impliziten Sozialvertrag nicht gefährden wollten. Diese Strategie mündete in einer Einkommenspolitik, welche stillschweigend Konfrontation mit den Gewerkschaften scheute. Notwendige institutionelle Reformen des Arbeitsmarktes, insbesondere eine Einschränkung der Gewerkschaftsmacht, welche den Weg zu Produktivitätsfortschritten eröffnet hätten, wurden deswegen nicht durchgeführt.

Die stagnierende Wirtschaftslage erschwerte die Kooperationsbedingungen der Sozialpartner, und deren Beziehungen verschlechterten sich. Spätestens Anfang der 70er Jahre, mit der ersten Ölpreiserhöhung durch die OPEC, stellten zunehmende Inflation und Arbeitslosigkeit, niedrige Produktivität, eine Verschlechterung des Außenbeitrags und eine Verlangsamung des Wachstums die Effektivität der Kooperation der Sozialpartner ernsthaft in Frage. ${ }^{286}$ Die Gewerkschaften waren nicht bereit, im Rahmen von einkommenspolitischen Vereinbarungen mehrere Perioden ohne vollständigen Teuerungsausgleich bzw. Reallohnerhöhungen zu akzeptieren. 1974 scheiterte die Konservative Regierung unter Edward Heath beim Versuch, mit der Einkommenspolitik die Preis-Lohn-Spirale zu durchbrechen.

Die nachfolgende Labour Regierung unter James Callaghan setzte 1974 die bisherige wirtschaftspolitische Strategie zunächst einmal fort. In der Rezession versuchte sie anhand einer Defizitpolitik, welche sich vor allem aus großen Lohnerhöhungen im öffentlichen Dienst und staatlichen Investitionsprojekten zusammensetzte, die Nachfrage anzukurbeln. ${ }^{287}$ Der erhoffte Aufschwung blieb jedoch aus. Wie das theoretische Ergebnis im ersten Kapitel, Abschnitt II erwarten ließ, führte die schuldfinanzierte Erhöhung der Staatsausgaben statt dessen zu rapide anwachsenden Budgetdefiziten, die zunehmend durch Auslandsverschuldung gedeckt werden mußten. Massive Kapitalflucht komprimierte die Finanzierungsschwierigkeiten für den Staat, bis 1976 der Druck auf die Wechselkurs- und Zinsentwicklung die Regierung zwang, sich für einen Überbrückungskredit an den Internationalen Währungsfonds (IWF) zu wenden. Der IWF setzte im Rahmen seiner Konditionalität u. a. der staatlichen Kreditfinanzierung strikte Grenzen. Danach stabilisierte sich die

${ }^{286}$ Brittan, (1983), S. 187ff.

287 Allgemein zu den Regierungen zwischen 1974-79 siehe Caimcross (1992), S. 201ff. und Britton (1991), S. 18ff. Zur damaligen Ausgabenpolitik, siehe Jackson (1991/, S. 74ff 
Wirtschaft für kurze Zeit. Das Budgetdefizit sank von 9,6\% des BIP 1975 auf 4,9\% 1978 , die Inflationsrate fiel von $27,1 \%$ auf $11,1 \%$, und die reale Wachstumsrate kehrte sich von $-0,9 \%$ in $3,6 \%$ um.

Gleichwohl zerbrach im sogenannten „Winter of Discontent“ 1978/79 der wirtschaftliche Konsens erneut am Widerstand der Gewerkschaften, die sich weigerten, ihre Lohnforderungen an die im Rahmen der Einkommenspolitik vorgeschlagenen $5 \% \mathrm{zu}$ halten. Die daraus resultierenden Auseinandersetzungen führten im Mai 1979 zu Neuwahlen und einem Regierungswechsel.

\section{Die schuldenpolitische Konzeption der Konservativen Regierung}

\section{1) Die Konservative Interpretation der Wirtschaftsentwicklung}

Die neugewählte Konservative Regierung versprach Veränderung. Nach ihrer Auffassung lag Großbritanniens wirtschaftliche Schwäche vor allem in der fortdauernden Inflation, welche den Staat auf Kosten der „Kapitalisten“ und „Kleinsparer“ zugleich bereicherte, an der überhöhten staatlichen Kreditaufnahme und Steuerbelastung der Bürger, die zur Finanzierung der öffentlichen Hand benötigt wurden, und an der staatlichen Regulierung der Wirtschaft, welche die Handlungsfreiheit einschränkte und die Innovationsbereitschaft hemmte. ${ }^{288}$ Ihre Interpretation der britischen Wirtschaftsschwäche - oder der „britischen Krankheit“, wie sie genannt wurde - war stark von der Lehrmeinung geprägt, die im weitesten Sinne Monetarismus genannt wird. ${ }^{289}$

Grundlegende Ursache für die Ungleichgewichte auf makroökonomischer Ebene war nach Ansicht der Konservativen Regierung die finanzielle Mißwirtschaft der Staatsfinanzen, deren Defizite durch erhöhte Kapitalüberschüsse entweder vom Privatsektor oder vom Ausland gedeckt werden mußten. Wenn der private Sektor seinen Kapitalüberschuß erhöhen sollte, bedeutete dies entweder steigende Ersparnisse oder eine Senkung der Investitionen. Paßte sich das Sparen dem erhöhten staatlichen Finanzierungsbedarf nicht ausreichend an, wurden Aktivitäten im Pri-

\footnotetext{
288 Siehe hierzu Walters (1983) S. 13f.

${ }^{289}$ Obwohl der Monetarismus eine breite Palette an Ansichten über wirtschaftliche Zusammenhänge umfaßt, sind allen gemeinsam der Glaube an eine langfristig stabile Beziehung zwischen Geldmengenwachstum und Wachstum des nominellen Einkommens, an einen langfristigen Zusammenhang zwischen Geldangebot und Budgetdefizit und an die Selbststabilisierungskräfte der Wirtschaft, die eine effiziente Allokation bei voller Ausnutzung der Ressourcen gewährleisten. Siehe z. B. Laidler (1975). 
vatsektor verdrängt. Wichtiger war nach Ansicht der Regierung jedoch die Finanzierung der öffentlichen Defizite durch den Erwerb von britischem Finanzvermögen durch das Ausland.

Bekanntlich entspricht der Nettoerwerb von britischem Finanzvermögen durch Ausländer gleichzeitig der Finanzierung des Leistungsbilanzdefizits. Die Konservative Regierung nahm an, daß die inländische Sparquote in der Regel ziemlich konstant blieb ${ }^{290}$ und eine Erhöhung des staatlichen Defizits sich deshalb in einer entsprechenden Zunahme des Leistungsbilanzdefizits widerspiegelt. Zum einen wurde die britische Exportindustrie verdrängt, zum anderen entstand ein Importsog, der die durch das Defizit gebildeten Ressourcen absorbierte. Im Laufe der Zeit - vor allem in den 70er Jahren - zeigte sich aber das Ausland immer weniger bereit - bzw. nur zu immer härteren Bedingungen - die öffentlichen Kredite zu finanzieren. Neben dem oben beschriebenen Ungleichgewicht des Außenbeitrags kam es dann auch verstärkt zum „Crowding Out“ des inländischen Privatsektors.

Die öffentlichen Finanzen waren nach Ansicht der neuen Regierung auch maßgeblich für die hohe Inflationsrate im Vereinigten Königreich verantwortlich. Erstens mußte zur Stabilisierung des Wechselkurses die Zentralbank den Devisenangebotsüberhang gegen Inlandgeld aufkaufen, was die Geldmenge erhöhte (1. Kapitel, Abschnitt IV.5). Zweitens, wie unten gezeigt wird, ist das öffentliche Defizit selbst eine Komponente des Geldangebots und demzufolge nährten steigende Defizite dessen Wachstum. Drittens führten die staatlichen Defizite zu steigenden Zinsen. Folgendes Zitat aus dem Haushaltsplan 1979 faßt nach Ansicht der Konservativen Regierung die Wirkungen hoher staatlicher Kreditaufnahme zusammen:

„High government borrowing has fuelled inflation, complicated the task of controlling the money supply, raised interest rates, and thus denied the wealth creating sector's some of the external finance they need for expansion." ${ }^{\text {291 }}$

2) Die Konservative Lösung - „a spoonful of courageous medicine“6292

Die Konservative Regierung setzte neue Ziele für die makroökonomische Wirtschaftspolitik - wie im ersten Haushaltsplan der Konservativen Regierung von November 1979 formuliert wurde:

\footnotetext{
${ }^{290}$ Beziehungsweise sollte sie bei stabilen Inflationsraten konstant bleiben, hierzu Walters (1985), S. $148 \mathrm{f}$.

${ }^{291}$ HMSO (1979), zitiert in Jackson (1980), S. 66.
}

292 Zitiert nach Alan Walters. 
„First, to bring down the rate of inflation. To achieve this it is essential to contain and reduce progressively the growth of the money supply. This means that government borrowing must, in turn, be controlled. It is a main determinant of monetary growth. Second to restore incentives. This means that the government must hold down and, if possible, reduce taxes particularly on incomes. Third to plan for spending which is not only compatible with the necessary objectives for taxation and borrowing but is also based on the realistic assessment of the prospects for economic growth.“293

Ein Wirtschaftsprogramm wurde vorgestellt, welches sich radikal von der vorigen Keynesianischen Konzeption unterschied und dessen budgetpolitische Komponenten sich wie folgt zusammenfassen lassen:

- Vorrangiges Ziel war die Verringerung der Inflationsrate durch eine restriktive, an einem Geldmengenziel orientierte Geldpolitik,

- begleitend hierzu war eine Senkung der staatlichen Kreditaufnahme beabsichtigt und ein Abbau der Staatsverschuldung,

- eine Umstrukturierung der Steuerlast sollte durch eine Verringerung der direkten Steuern und eine Erhöhung der indirekten Steuern erreicht werden; längerfristig waren Steuersenkungen vorgesehen,

- schließlich wollte die Regierung durch Ausgabenkürzungen und die Privatisierung von öffentlichen Unternehmen den Umfang des öffentlichen Sektors senken sowie dessen Effizienz erhöhen.

Im Vergleich $\mathrm{zu}$ den vorherigen Konzeptionen unterschied sich die Konservative Strategie insbesondere in drei Punkten. ${ }^{294}$ Erstens kehrte sie sich von der Vorstellung ab, Wachstums- und Beschäftigungsziele anhand der Stabilisierungspolitik erreichen zu wollen. Zweitens distanzierte sie sich von jeglicher Art von Einkommenspolitik zur Inflationsbekämpfung. Drittens erstrebte sie eine Reduktion des staatlichen Einflusses auf das Wirtschaftsgeschehen in allen Bereichen.

Neu bei der Formulierung des Programms war u. a. der Glaube an eine Wirtschaftspolitik, welche die Erwartungen der Wirtschaftssubjekte berücksichtigt. Denn aus der Sicht von Patrick Minford vom geldpolitischen Ausschuß der Regierung:

${ }^{293}$ HMSO (1979), zitiert in Jackson (1980), S. 66f.

${ }^{294}$ Vgl. hierzu Hennings (1985), S. 145f. sowie Begg (j987), S. 39f. 
"The overwhelming problem we face in the U.K. economy is that of breaking, once and for all, the inflation psychology." ${ }^{295}$

Zur Beseitigung der Inflationserwartungen war eine Strategie notwendig, welche langfristig ausgelegt und vor allem glaubwürdig war. Die Konservative Regierung war sich bewußt, daß ihre radikale Politikwende nicht ohne Widerstand hingenommen würde, daß jedoch die Erreichung von gesteckten Zielen unbedingt notwendig für ihre Glaubwürdigkeit und demzufolge auch für ihren Erfolg war. Deswegen war sie fest entschlossen, ihre makroökonomische Strategie auch gegen größte Widerstände ohne Abweichungen durchzusetzen.

\section{3) Die mittelfristige Finanzstrategie}

Im Mittelpunkt der makroökonomischen Konzeption der Konservativen Regierung stand die "mittelfristige Finanzstrategie“ (medium-term financial strategy oder MTFS). Diese stellte ein komplementäres Zielbündel aus restriktiven geld- und fiskalpolitischen Maßnahmen im Rahmen eines mehrjährigen Programmes dar. ${ }^{296}$ So bestand die Fiskalpolitik für einen insgesamt vierjährigen Zeitraum in jährlichen Zielvorgaben für die Budgetdefizite als Anteil am BIP, welche im Zeitablauf sukzessive abnahmen. In ähnlicher Weise und über den gleichen Zeitraum hinweg formulierte die Geldpolitik Ziele für die Wachstumsrate der Geldmenge.

Die MTFS wurde zum ersten Mal 1980 formuliert und seitdem jährlich im Haushaltsplan verlängert. Ihre fundamentale Logik basiert auf der Quantitätstheorie:

$$
\mathrm{MV}=\mathrm{PY} .
$$

Unter der Annahme, daß die Umlaufgeschwindigkeit des Geldes voraussehbar und relativ stabil ist, besteht ein enger Zusammenhang zwischen der Geldmenge und dem nominalen Einkommen. Nimmt man ferner an, daß das reale Einkommen kurzfristig konstant ist, muß sich eine Geldmengenerhöhung automatisch auf eine Erhöhung des Preisniveaus übertragen. Deswegen kann der Staat durch Steuerung der Geldmengen-Wachstumsrate die Inflationsrate kontrollieren.

\footnotetext{
295 HMSO (1980a), S. 131.
}

${ }^{296}$ Im Unterschied zu Deutschland lagen bis Mai 1997 in Großbritannien alle strategischen geldpolitischen Entscheidungen in der Hand der Regierung. Die praktische Ausübung der Geldpolitik oblag zwar der Notenbank (Bank of England). Allerdings war diese politisch nicht unabhängig, sondern befolgte die Anweisungen des Schatzkanzlers der Regierung. 
Aufgrund der starken Inflationserwartungen der $80 \mathrm{er}$ Jahre gründete die MTFS nicht auf der Quantitätstheorie in ihrer einfachsten Form, sondern auf der realistischeren Vorstellung, $\mathrm{da} \beta$ sich eine fallende Wachstumsrate der Geldmenge nicht nur auf die Inflationsrate, sondern auch auf das reale Wachstum auswirken würde. Nach Anpassung der Inflationserwartungen an die neue restriktive Geldpolitik und der entsprechenden Senkung der Inflationsrate, könnte jedoch höheres Realwachstum bei der vorgesehenen Wachstumsrate der Geldmenge akkommodiert werden. Um die rasche Anpassung der Inflationserwartungen an die neue Politik zu fördern, wurde das Geldmengenziel durch die Kontrolle eines einzigen Geldmengenbegriffs innerhalb eines Zielkorridors angestrebt. Denn nach Ansicht der Regierung würde mehr als ein Geldmengenziel:

„make it much more difficult for the market and the public to appraise the determination of the authorities to meet their monetary objectives.“297

$\mathrm{Zu}$ diesem Zwecke wurde ein breit definiertes Geldmengenaggregat in der sogenannten Abgrenzung Sterling M3 gewählt. ${ }^{298}$ Entsprechend der Konservativen Überzeugung von der Effizienz ungestörter Marktprozesse sollte dieses Geldmengenziel „without excessive reliance on interest rates" erreicht werden, ${ }^{299}$ sondern durch einen stetig und langsam sinkenden Wachstumspfad für das Budgetdefizit. ${ }^{300}$

Der unterstellte Zusammenhang zwischen dem Budgetdefizit und der Geldmenge läßt sich vor dem Hintergrund einer langen Tradition der Kreditrationierung als Bestandteil der Geldpolitik im Vereinigten Königreich verstehen. ${ }^{301}$ Entsprechend diesem Usus orientierte sich die Geldmengenkontrolle an der Kreditnachfrage bei den Banken und nicht an der Nachfrage nach Depositen. Das Budgetdefizit stellt als staatliche Kreditnachfrage eine Komponente der gesamten Kreditnachfrage dar, weshalb die Regierung glaubte, mit der Kontrolle dieser Komponente die Entwicklung der gesamten Kreditvergabe und damit der Geldmenge beeinflussen zu können. Um die Wirkungsweise der beabsichtigten Kontrolle besser zu verstehen, sind folgende Definitionen hilfreich. ${ }^{302}$

\footnotetext{
${ }^{297}$ HMSO (1980c), S. iv.

${ }^{298}$ Es handelt sich um den Bargeldumlauf zuzüglich sämtlicher Bankeinlagen (inklusive Termingelder und Depositenzertifikate), soweit sie von britischen Nichtbanken gehalten werden und auf Pfund Sterling lauten.

${ }^{299}$ HMSO (1980b), S. 9.

300 Buiter und Miller (1981), S. 340.

${ }^{301}$ Hierzu Dow und Saville (1988), insbesondere S. 153ff.

302 Siehe HMSO ( 1980b).
} 
$£ \mathrm{M3} \equiv$ Bargeldumlauf + alle Einlagen in Sterling des Inlands bei inländischen Banken.

Die Bilanz des Bankensektors im Vereinigten Königreich läßt sich vereinfacht folgendermaßen darstellen:

\begin{tabular}{|l|l|}
\hline \multicolumn{1}{|c|}{ Aktiva } & \multicolumn{1}{c|}{ Passiva } \\
\hline Sterling-Darlehen an den Privatsektor & Sterling-Einlagen des Inlands \\
\hline Sterling-Darlehen an den Staatssektor & Sterling-Einlagen des Auslands \\
\hline Sterling-Darlehen an das Ausland & Einlagen in Fremdwährungen \\
\hline Darlehen in Fremdwährungen & Andere Passiva \\
\hline
\end{tabular}

Daraus folgt die Definition von Änderungen von $£ \mathrm{M} 3$ in Abhängigkeit der Aktivitäten des Bankensektors:

$\Delta £ \mathrm{M} 3 \equiv \Delta$ Bargeldumlauf

$+\Delta$ Sterling-Darlehen an die privaten und öffentlichen Sektoren

$+\Delta$ Sterling-Darlehen an das Ausland

- $\Delta$ Sterling-Einlagen des Auslands - $\Delta$ Netto-Transaktionen in Fremdwährungen

- $\Delta$ andere Passiva.

Das Budgetdefizit läßt sich folgendermaßen definieren:

$$
\begin{aligned}
\text { Budgetdefizit } \equiv & \Delta \text { Bargeldumlauf } \\
+ & \text { Darlehen von inländischen Nichtbanken und dem } \\
& \text { Ausland } \\
+ & \Delta \text { Sterling Darlehen der Banken an den öffentlichen } \\
& \text { Sektor. }
\end{aligned}
$$

Folglich kann die Kreditvergabe der Banken an den öffentlichen Sektor dargestellt werden als das Budgetdefizit abzüglich aller anderen Verkäufe von öffentlicher Schuld. Durch Einsetzen dieser Definition in die £M3 Gleichung und mit einigen Vereinfachungen ergibt sich die Veränderung der Geldmenge £M3 in Abhängigkeit des Budgetdefizits. ${ }^{303}$

${ }^{303}$ Eine ausführliche Ableitung von $£ \mathrm{M} 3$ und ihrer Finanzierungskomponenten befindet sich in Temperton (1986), S. 29ff. 
Folgende Identität wird von der Bank of England regelmäßig veröffentlicht: ${ }^{304}$
$\triangle £ \mathrm{M} 3 \equiv$ Budgetdefizit
- Verkäufe von öffentlicher Schuld an inländische Nichtban- ken
$+\Delta$ Sterling Darlehen der Banken an den Privatsektor
+ Netto-Transaktionen in Fremdwährungen und mit dem Aus- land
- $\Delta$ andere Passiva.

Die ersten drei Termini stellen die Komponente des inländischen Kreditwachstum dar. Wenn es dem Staat gelingt, seine Kreditnachfrage zu reduzieren, dann sinkt ceteris paribus das Geldmengenwachstum.

In der Realität ist allerdings die Korrelation zwischen dem Wachstum von £M3 und dem Budgetdefizit nicht sehr eng, weil die ceteris paribus-Annahme der Konstanz anderer Kreditkomponenten nicht gilt. ${ }^{305}$ Es wurde jedoch geglaubt, daß anhaltend hohe öffentliche Defizite sich letztendlich in einer hohen Wachstumsrate der Geldmenge niederschlagen würden. ${ }^{306}$

Die MTFS war der zentrale Bestandteil der von der Konservativen Regierung versprochenen Veränderung: ${ }^{307}$ Die Stellung der Fiskalpolitik ergab sich aus der monetären Zielsetzung und nicht aus einer Nachfragepolitik, welche auf die realen Größen des Outputs und der Beschäftigung abstellte. Die Zielvorgabe bis zu vier Jahren sollte der Strategie dahingehend Glaubwürdigkeit verleihen, da $B$ die Ziele nicht kurzfristigen konjunkturellen oder politischen Überlegungen zum Opfer fal-

\footnotetext{
${ }^{304}$ Siehe Bank of England Quarterly Bulletin, Tabellen 11.3. und 12.1.

${ }^{305}$ Beispielsweise führte Anfang der 80er Jahre die schrittweise Liberalisierung der Bankenindustrie zu großen Schwankungen bei der Kreditvergabe der Banken. Diese konkurrierten stark mit den Bausparkassen, vor allem um die Hypotheken. Die geldschaffenden Aktivitäten der Bausparkassen zählten in den 80 er Jahren nicht zu $£ \mathrm{M} 3$, so daß wettbewerbsbedingte Verschiebungen der Einlagen zwischen Banken und Sparkassen sich in der Entwicklung von fM3 niederschlugen. Auch die Liberalisierungen des Zahlungsverkehrs und der Konsumentenkreditgesetze, sowie der Verkauf öffentlicher Wohnungsbauten, wirkten sich auf $£ \mathrm{M} 3$ aus.

${ }^{306}$ Vgl. hierzu das obige Argument von Sargent und Wallace (1981) im Abschnitt II, S. 54.

307 Siehe Buiter und Miller $(1981,1983)$ für eine ausfuhrliche Diskussion der MTFS und ihrer Konsequenzen. 
len könnten. Endziel der ersten Strategie von 1980 war ein jährliches Geldmengenwachstum von $6 \%$ und eine Defizitquote von $1,5 \%$ bis $1984 .{ }^{308}$

Diese Vorgaben erfuhren in den folgenden Jahren allerdings einige Modifikationen. Mehrmals wurden neue Zwischenziele für die Geldpolitik formuliert, und auch die Endziele der Fiskalpolitik änderten sich. Ab 1982 wurde der Wechselkurs als zusätzliche Zwischenzielgröße in die Strategie integriert, 1984 die Geldbasis M0 ${ }^{309}$ und 1986 das nominale BIP. Die Wechselkursentwicklung wurde seit 1982 als Orientierungsgröße bekanntgegeben und während der Mitgliedschaft im Wechselkursmechanismus von Oktober 1990 bis September 1992 als explizites Ziel verfolgt. Seitdem wird auch das Endziel der Inflationsrate selbst angegeben. ${ }^{310}$

Während in den ersten Jahren die MTFS in erster Linie die Geldpolitik unterstützte, traten zunehmend die Zielvorstellungen für den öffentlichen Schuldenbedarf und die Senkung der Staatsverschuldung in den Vordergrund. ${ }^{311} 1986$ gab die Regierung als Ziele eine stabile Schuldenquote und Preisstabilität bekannt, was eine Defizitquote von $1 \%$ bedeutete. Schließlich wurde 1988 ein mittelfristiger PSBR von Null als Ziel bekanntgegeben. Im Unterschied zur früheren Formulierung der MTFS wurden jedoch kurzfristige Abweichungen vom Ziel im Konjunkturverlauf eingeräumt. Im Aufschwung sollten Budgetüberschüsse erwirtschaftet werden und die PSBR wurde in die PSDR (Public Sector Debt Repayment - öffentliche Schuldentilgung) umbenannt.

\section{4) Die verwendeten Strategien zur Senkung des Budgetdefizits}

Die MTFS setzte nicht nur Ziele für das Budgetdefizit, sondern umfaßte auch Pläne für ihre Verwirklichung. Dafür standen, wie die Analyse der Budgetbeschränkung im vorigen Kapitel (Abschnitt I) gezeigt hat, vier Finanzierungsquellen zur Verfügung: Inflation, Erhöhung der Steuereinnahmen, Senkung der Ausgaben oder Einnahmen aus der Veräußerung von öffentlichem Vermögen.

Die MTFS erlaubte selbstverständlich keine Budgetfinanzierung durch die Inflation. Entsprechend ihrem Glauben an die negativen Auswirkungen der Besteuerung auf die Effizienz der freien Marktkräfte kamen für die Konservative Regierung Steuererhöhungen ebenfalls nicht in Frage. Im Gegenteil, sie strebte eine Senkung

\footnotetext{
${ }^{308}$ Budd (1985), S 19.

${ }^{309}$ M0 = Bargeldumlauf + Guthaben des Bankensektors bei der Zentralbank.

${ }^{310}$ Artis (1988), S. 16 und King (1994), S. 115 f.

${ }^{311}$ Buiter und Miller (1983), S. 364. Budd (1988), S. 5 f.
} 
der Steuerlast an. Damit verblieben als Finanzierungsoptionen noch Ausgabensenkungen oder Einnnahmen aus der Privatisierung.

\section{a) Ausgabensenkungen}

Wenn die geplante Senkung der Neuverschuldung nicht durch Inflation oder Steuererhöhungen erfolgen sollte, dann mußte eine Hauptlast auf Einsparungen bei den Ausgaben liegen. In den Anfangszeiten der Konservativen Regierungen waren denn auch große Ausgabenkürzungen geplant.

„We shall look for major savings in the cancellation of Socialist programmes; an end to nationalisation; the reduction of indiscriminate subsidies ... We shall also press for a major increase in the efficiency of local government including much less duplication with Whitehall, [dem Zentralstaat, J.S.] we shall be looking for very substantial savings here over a five year period.“"312

In bezug auf die Ausgaben für den Wohlfahrtsstaat betonte die Regierung jedoch, daß die Einsparungen keine Leistungseinbuße zur Folge haben sollten. Ihre Strategie lautete:

,... not being a prescription for poorer social provision, it is a recipe for better housekeeping in all the public services. If we are to maintain standards of services we must root out waste and unnecessary bureaucracy.“313

Im Rahmen der ersten MTFS von 1980 bis 1984 war eine jährliche Senkung der realen Staatsausgaben um 1\% vorgesehen. Wie die Analyse im nächsten Kapitel zeigen wird, wurde dieses Ziel nicht erreicht. Deswegen gab nach 1984 die Regierung die Vorstellung realer Ausgabensenkungen auf und verfolgte statt dessen eine sogenannte „Konsolidierungsstrategie“, wonach die budgetierten Staatsausgaben real konstant gehalten werden sollten. Eine Senkung der Ausgabenquote von $42,5 \%$ auf $34,5 \%$ bis 1993 sollte durch ein Wachstum des Sozialprodukts erreicht werden. ${ }^{314}$ Dieses Ziel wurde 1993 erneut modifiziert. Die neue Ausgabenpolitik plante einen jährlichen Anstieg der Ausgaben um real 1,5\%, was unter der Annah-

\footnotetext{
312 Conservative Central Office (1977), S. 11.

${ }^{313}$ Ebenda.

${ }^{314}$ Budd et al. (1985), S. 23 sowie Burton (1985), S. 29
} 
me des Trendwachstums von 2\% eine geringe Senkung der Ausgabenquote bedeutete. ${ }^{315}$

\section{b) Die Privatisierungspolitik}

Pläne für die Privatisierung von öffentlichen Unternehmern tauchten im Konservativen Wahlmanifest 1979 nicht auf. Erst in der zweiten Wahlperiode nach 1983 entwickelten sie sich allmählich aus der traditionellen Konservativen Unterstützung von Privateigentum zu einem wichtigen Eckstein der angebotsorientierten Politik. Ab 1983 wurde die Privatisierung dann intensiv auf zentralstaatlicher Ebene verfolgt ${ }^{316}$. Auf lokaler Ebene wurden durch die Veräußerung von sozialen Wohnungsbauten auch die Vermögensbestände der Gemeinden abgebaut. Die aus den Verkäufen gewonnenen Mittel, verwendet zur Finanzierung der öffentlichen Hand, stellten einen zusätzlichen Anreiz für die weitere Entwicklung eines umfassenden Privatisierungsprogramms dar.

\section{Die britische Budgetpolitik im Lichte der staatlichen Budgetbeschränkung}

Im folgenden wird im Rahmen der staatlichen Budgetbeschränkung gezeigt, wie die MTFS gestaltet war und wie diese Gestaltung sich auf die Budgetfinanzen auswirken sollte. Als erstes wird die britische Abgrenzung des Budgetdefizits erläutert. Danach wird die Bedeutung der Ausgaben als endogene Variable in der Budgetbeschränkung aufgezeigt. Schließlich wird die Strategie der Inflationsbekämpfung durchleuchtet.

\section{1) Die Abgrenzung des Budgetdefizits im Vereinigten Königreich}

Die öffentliche Diskussion um das Budgetdefizit wird traditionellerweise nicht als eine Gegenüberstellung von Einnahmen und Ausgaben geführt. ${ }^{317}$ Vielmehr liegt ihr eine Vorstellung des öffentlichen Entsparens (bzw. Sparens) im Zeitverlauf zu-

\footnotetext{
315 CSO (1993), S. $11 \mathrm{ff}$.

${ }^{316}$ Ausführlicher im 4. Kapitel, Abschnitt II.2. Eine Auflistung der wichtigsten Privatisierungen befindet sich im Anhang.

${ }^{317}$ Vgl. Gleichung 2, im zweiten Kapitel I.2, S. 49. 
grunde, das in der volkswirtschaftlichen Gesamtrechnung als eine Zunahme (Abnahme) des staatlichen Anteils an Geld- und Schuldbeständen definiert wird. Die folgende Gleichung zeigt das öffentliche Entsparen oder den sogenannten öffentlichen Kreditbedarf („Public Sector Borrowing Requirement“ - PSBR). Diese Kennzahl gilt als wichtigster finanzpolitischer Parameter im Vereinigten Königreich.

$$
P S B R \equiv P_{t} G_{t}+T r_{t}-T_{t}+i_{t-1} B_{t-1}-\left(A_{t}-A_{t-1}\right)=\left(M_{t}-M_{t-1}\right)+\left(B_{t}-B_{t-1}\right) .
$$

Auffallend bei der Abgrenzung des PSBR ist es, daß die Privatisierungserlöse implizit als negative Ausgaben oder im Sinne Musgrave et al. als ein finanzwirtschaftspolitisches Instrument behandelt werden. ${ }^{318}$ Dies hat Konsequenzen für die Budgetfinanzierung, wie im nächsten Abschnitt gezeigt wird.

\section{2) Die Bedeutung der Staatsausgaben bei der mittelfristigen Finanzstrategie}

Um die budgetpolitischen Gestaltungsmöglichkeiten der MTFS näher zu betrachten, sollen die Finanzierungsparameter des PSBR kurz diskutiert werden. Es ist offensichtlich, daß eine Verwendung der Seignorage $\left[\left(M_{t}-M_{t-1}\right)\right.$ definiert im Kapitel II] zur Budgetfinanzierung ausgeschlossen ist, da das übergeordnete Ziel eine Inflationsbekämpfung ist. Die Höhe der Neuverschuldung $\left(B_{t}-B_{t-1}\right)$ wurde im Rahmen der MTFS festgelegt und steht nur in dieser Höhe als Finanzierungsquelle zur Verfügung.

Auf der linken Seite der Gleichung sind die Steuereinnahmen $\left(T_{t}\right)$. Ziel der Regierung war es, die Steuerquote zu senken, weshalb Steuererhöhungen zur Einhaltung der Zielsetzung für die Neuverschuldung nicht eingesetzt werden konnten. Die Privatisierungserlöse $\left(A_{t}-A_{t-1}\right)$ bringen zusätzliche Einnahmen für den Staat. Ihre Höhe in einzelnen Jahren ist aufgrund administrativer und politischer Rahmenbedingungen im Zusammenhang mit der Verkaufsstrategie beschränkt. Sie kann jedoch für einige Jahre einen guten Anteil zur Budgetfinanzierung betragen und damit den budgetpolitischen Spielraum erweitern.

Schließlich gibt es die Staatsausgaben, welche sich aus den nominalen Staatskäufen und den Transferzahlungen zusammensetzen $\left(P_{t} G_{t}+T_{t}\right){ }^{319}$ Wenn zunächst einmal von den Einnahmen der Privatisierungspolitik abgesehen wird, so bedeutet die Festlegung der Neuverschuldung im Rahmen der MTFS, zusammen mit dem Ziel der Steuersenkung, daß die Staatsausgaben insgesamt die einzige variable An-

${ }^{318}$ Musgrave, Musgrave und Kullmer (1994), S. 15.

${ }^{319}$ Von den exogenen Zinszahlungen auf der Staatsschuld wird abgeseheng-3/8-3-631-75199-2 
passungsgröße zur Erfüllung der Budgetbeschränkung waren. Darin sind die Transferzahlungen $\left(\mathrm{Tr}_{\mathrm{t}}\right)$ wegen der Verpflichtungen des Sozialstaats ${ }^{320}$ zumindest kurzfristig fest vorgegeben. Folglich blieben nur die Staatskäufe $\left(P_{t} G_{t}\right)$ als Anpassungsgröße der Budgetbeschränkung übrig. Mit anderen Worten: Mit dieser Gestaltung der MTFS wurde das Ausmaß der Staatskäufe durch die Strategie der Inflationsbekämpfung und das Ziel der Steuersenkung bestimmt und nicht nach den konventionellen Methoden der Präferenzoffenbarung oder Bedarfsschätzung. Einzig Einnahmen aus dem Verkauf von öffentlichem Vermögen konnten demnach den budgetpolitischen Spielraum vorübergehend erweitern.

\section{3) Die Auswirkungen der Inflation auf die Budgetbeschränkung}

Im zweiten Kapitel wurde gezeigt, daß dem Staat eine Inflation bei seinen Budgetfinanzen nützlich sein kann - auf Kosten der Gläubiger. Deswegen war es gerade für die Regierung wichtig, die Öffentlichkeit glaubhaft zu überzeugen, daß sie die Inflation nicht zur Finanzierung des Budgets ausnutzen wolle. In den folgenden Abschnitten soll erstens gezeigt werden, wie die MTFS eine Budgetfinanzierung aus der Inflation verhindern und zweitens, wie die Inflation sich auf die Entwicklung der Staatsausgaben auswirken sollte.

\section{a) Die Auswirkungen der Inflation auf das Defizitziel}

Die Ziele des öffentlichen Kreditbedarfs (PSBR) im Rahmen der MTFS wurden als nominale Größen formuliert. Die reale Wirkung der MTFS auf die Budgetfinanzen beruhte auf dem im vorigen Kapitel beschriebenen Entwertungseffekt der Inflation und läßt sich unter Berücksichtigung der konkreten Zielvorgaben und einiger vereinfachenden Annahmen veranschaulichen.

Folgende Tabelle gibt die Ziele der MTFS für das Geldmengenwachstum £M3 und den PSBR im Verhältnis zum BIP wieder, wie sie 1980 und dann erneut 1984 formuliert wurden. ${ }^{321}$ Wie oben bereits erläutert (Abschnitt III.3) wurde nach 1986/87 das Ziel $£ \mathrm{M} 3$ aufgegeben und das Geldmengenziel nur in der engen Abgrenzung M0 formuliert. Deswegen ist es sinnvoll, diese Darlegung der Wirkung der MTFS auf die Finanzjahre 1980/81 bis 1986/87 zu beschränken.

\footnotetext{
${ }^{320}$ Hierzu Andel (1990), S. 225 f.
}

${ }^{321}$ Die angegebenen Ziele wurden je nach wirtschaftlicher Entwicklung kurzfristig etwas revidiert. Davon wird an dieser Stelle abgesehen. 
Tabelle 3: Die MTFS, Geldmengenwachstum und der PSBR

\begin{tabular}{|l|c|c|c|}
\hline Finanzjahr & fM3 Wachstum (\%) & PSBR/BIP (\%) & Impliziertes Inflationsziel \\
\hline $1980 / 81$ & $7-11$ & 3,75 & $5-9$ \\
\hline $1981 / 82$ & $6-10$ & 3 & $4-8$ \\
\hline $1982 / 83$ & $5-9$ & 2,25 & $3-7$ \\
\hline $1983 / 84$ & $4-8$ & 1,5 & $2-6$ \\
\hline $1984 / 85$ & $6-10$ & 2 & $4-8$ \\
\hline $1985 / 86$ & $5-9$ & 2 & $3-7$ \\
\hline $1986 / 87$ & $4-8$ & 2 & $2-6$ \\
\hline
\end{tabular}

Quelle: Temperton (1986), S. 11

Die Berechnungen für die Zielgrößen der MTFS unterstellten eine reale Wachstumsrate zwischen $1,75 \%$ und $2,5 \%$. Vereinfachend wird hier ein Durchschnittsoder Trendwert von $2 \%$ angenommen. ${ }^{322}$ Ferner wird vorausgesetzt, daß die Quantitätstheorie gilt und Änderungen der Geldmenge sich in der Inflationsrate widerspiegeln. Daraus folgt, daß beispielsweise für das Finanzjahr 1980/81 der Zielkorridor für das Geldmengenwachstum von 7-11\% nach Abzug des Realwachstums einer erwarteten Inflationsrate von 5-9\% entsprach (3. Kolonne der Tabelle). Das geplante Budgetdefizit lag bei 3,75\%.

322 Ein realer Wachstumstrend von $2 \%$ liegt den langfristigen Haushaltsplänen zugrunde, vgl. CSO (1993), S. 12. 
Die Beziehung läßt sich graphisch darstellen:

Abbildung 11: Die Ziele der MTFS von 1980/81 bis 1986/87

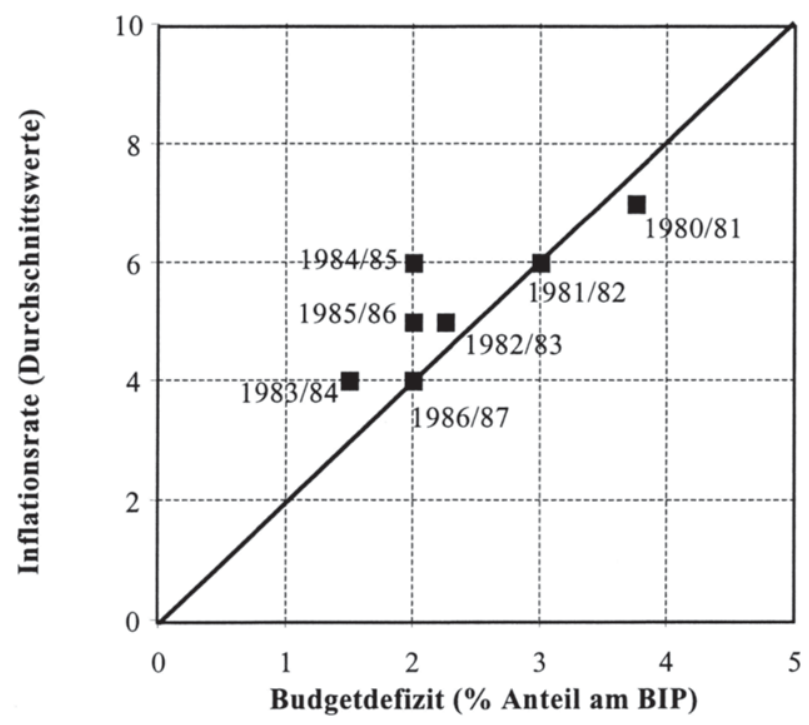

Eine doppelt so enge Skalierung für die Inflationsrate wurde gewählt, weil dann eine $45^{\circ}$-Linie die Höhe der Inflationsbereinigung ( $\pi \mathrm{B}$ wie sie im Anhang II abgeleitet wurde), ausgedrückt als Anteil am BIP, für eine Schuldenquote von $50 \%$ wiedergibt. Dies ist im Vereinigten Königreich der 80er Jahre annähernd der Fall gewesen (vgl. Abbildung 10). Somit stellt diese $45^{\circ}$-Linie gerade den geometrischen Ort von realem Budgetausgleich dar. Der Bereich links von der $45^{\circ}$-Linie bedeutet einen realen Budgetüberschuß, derjenige rechts davon ein reales Budgetdefizit.

Die Ziele der MTFS streuen alle nah um die $45^{\circ}$-Linie. Die Schuldentilgung infolge Inflation gleicht in etwa die geplanten nominalen Budgetdefizite aus, so daß die 
geplanten realen Budgetdefizite annähernd bei Null liegen. Mit anderen Worten, die MTFS schien eine inflationsbereinigte Parallelpolitik verfolgen zu wollen. ${ }^{323}$

Um die geplanten mit den realisierten Werten zu vergleichen, gibt die Tabelle 4 die tatsächlichen Inflationsraten des BIP und den jährlichen Kreditbedarf des öffentlichen Sektors an.

Tabelle 4: Inflation und der PSBR 1980-1994

\begin{tabular}{|c|c|c|c|}
\hline Jahr & Inflation ${ }^{324}(\%)$ & PSBR/BIP (\%) & Inflationsbereinigtes Budgetdefizit \\
\hline 1980 & 19,4 & 5,1 & $-4,6$ \\
\hline 1981 & 11,5 & 4,2 & $-1,6$ \\
\hline 1982 & 7,5 & 1,7 & $-2,1$ \\
\hline 1983 & 5,3 & 3,8 & 1,2 \\
\hline 1984 & 4,5 & 3,2 & 1 \\
\hline 1985 & 5,7 & 2,1 & $-0,8$ \\
\hline 1986 & 3,3 & 0,6 & $-1,1$ \\
\hline 1987 & 5,1 & $-0,3$ & $-2,9$ \\
\hline 1988 & 6,0 & $-2,5$ & $-5,5$ \\
\hline 1989 & 7,1 & $-1,8$ & $-3,4$ \\
\hline 1990 & 6,4 & $-0,4$ & $-3,6$ \\
\hline 1991 & 6,5 & 1,3 & $-2,0$ \\
\hline 1992 & 4,3 & 4,8 & 2,7 \\
\hline 1993 & 3,3 & 6,7 & 5,1 \\
\hline 1994 & 2,1 & 5,5 & 4,5 \\
\hline
\end{tabular}

${ }^{323}$ Die Logik der Zielvorgaben der MTFS bleibt umstritten. Interpretationen bieten z. B. Minford (1980), Budd und Burns (1981), Buiter und Miller (1981, 1983), Miller (1982), Walters (1985, 1986).

${ }^{324}$ BIP zu Marktpreisen (CSO), siehe statistischen Anhangth Safford - 978-3-631-75199-2 
So lag beispielsweise 1980 die Inflation bei $19,4 \%$ und der PSBR bei 5,1\%. Die Schuldentilgung infolge der Inflation machte die Hälfte von $19,4 \%$, d. h. 9,7\% des BIP aus. Folglich wurde entsprechend diesem Modell 1980 ein realer Budgetüberschuß von 4,6\% des BIP erwirtschaftet. Analog wurde für 1981 bei einer Inflationsrate von $11,5 \%$ ein nominales Budgetdefizit von $4,2 \%$ ausgewiesen. Das reale Ergebnis war ein Budgetüberschuß von 1,6\%. Die Werte bis 1994 sind graphisch in Abbildung 12 abgebildet.

\section{Abbildung 12: Die realisierte MTFS: Inflation und der PSBR 1980 bis 1986}

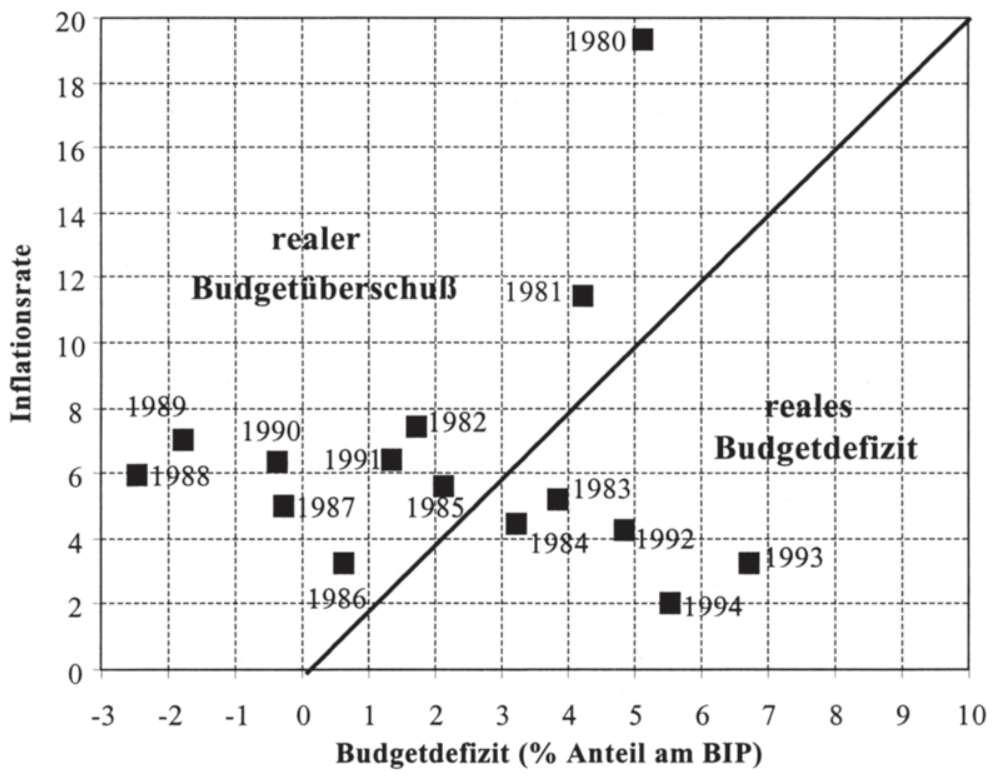

Es fällt sofort auf, daß entsprechend den Annahmen dieses Modells mit Ausnahme der Jahre 1983 und 1984 und den drei letzten Jahren reale Budgetüberschüsse erwirtschaftet wurden.

Dieses einfache Modell und die Zahlenbeispiele sind relativ grobe Näherungen. Wie im nächsten Kapitel gezeigt wird, liefern präzisere Berechnungen Werte, die leicht von diesen Zahlen abweichen. Dennoch veranschaulicht das Modell, wie die MTFS eine Verbindung zwischen Budgetdefizit und Inflationsrate erzeugte, und 
wie diese sich ausgewirkt hat. Gemäß den Zielen der MTFS wurde neu geschaffenes staatliches Finanzvermögen infolge einer Neuverschuldung durch die Inflation real vernichtet, so daß das reale Budgetdefizit annähernd ausgeglichen blieb. $\mathrm{Ab}$ weichungen vom Inflationsziel hatten bei Einhaltung der geplanten Neuverschuldung auch Folgen für die Staatsfinanzen. Lag beispielsweise die Inflationsrate höher als die vorausgesehene (oder das Budgetdefizit niedriger), fand eine automatische reale Schuldentilgung durch die Inflation statt. Für den Fall einer ungeplanten Deflation, wäre ein reales Budgetdefizit die Folge.

Die Auswirkungen der MTFS als fiskalpolitische Strategie sind vielerorts diskutiert worden ${ }^{325}$ und sollen hier nicht erörtert werden. Deutlich ist jedoch, wie die MTFS als Finanzstrategie eine makroökonomische Strategie der Schuldentilgung beinhaltete.

\section{b) Der Einfluß der Inflation auf die Staatsausgaben}

Im zweiten Kapitel wurde auch dargestellt, wie die Auswirkungen der Inflation von den Verteuerungsraten der einzelnen Ausgaben im Verhältnis zur gesamtwirtschaftlichen Inflationsrate abhängen. Die Konservative Regierung versuchte nicht, unter Berücksichtigung dieser Tendenz Einflu $B$ auf die einzelnen Ausgabekategorien zu nehmen. Vielmehr strebte sie an, das nominale Wachstum der Staatsausgaben insgesamt zu begrenzen. ${ }^{326}$ Die MTFS legte nominale Grenzen, sogenannte "Cash Limits“, für die Ausgaben fest, gesamthaft definiert als „Planning Total“ (PT). Diese Planungsgröße umfaßte die Ausgaben der zentralstaatlichen Ministerien und der staatlichen Unternehmen, die Zuweisungen an die lokalen Gebietskörperschaften und an einen Reserve-Fonds, sowie die eingenommenen Finanzmittel aus der Veräußerung von staatlichem Vermögen. Ihre Festlegung setzte bei der Haushaltsplanung eine Annahme über das reale Wachstum sowie über die Inflationsentwicklung voraus. Mit anderen Worten, es mußte in der vorigen Periode ( $t-1)$ die Höhe der zukünftigen Inflationsraten $\left(\mathrm{P}_{\mathrm{t}+\mathrm{x}}\right)^{327}$ prognostiziert werden. Das PT funktionierte dann in ähnlicher Weise wie das oben diskutierte Ziel für Neuverschuldung. Fiel die tatsächliche Inflation höher aus als die prognostizierte, dann mußte bei unveränderten nominalen Staatsausgaben der reale Wert der Ausgaben sinken. Unterschritt dagegen die tatsächliche Inflation die prognostizierte, dann wurde ein Raum für eine reale Erhöhung der Ausgaben geschaffen.

${ }^{325}$ Siehe unter vielen Buiter und Miller (1981, 1983), sowie Begg (1987).

${ }^{326}$ Hierzu Peacock (1985).

${ }^{327}$ Im Rahmen der MTFS $x=\{0,1,2,3\}$. 
Einige Ausgabenkategorien wurden von den Sparplänen ausgenommen. Die wichtigste Modifikation dabei war die Regelung der personellen Transferzahlungen. Diese wurden nicht mehr der Lohnentwicklung, sondern lediglich der Inflation angepaßt, womit das reale Wachstum unberücksichtigt blieb. Wenn die Anzahl der Transferempfänger gleich bleibt, dann nehmen die Transferzahlungen in einer wachsenden Wirtschaft als Anteil am BIP ab. Auch bei zunehmender Zahl von Transferempfängern wird so dem Kostenanstieg etwas Einhalt geboten. Zudem hoffte die Regierung, durch eine relative Abnahme der Transferleistungen im Verhältnis zur Lohnentwicklung positive Arbeitsanreize zu schaffen und damit ebenfalls Ausgaben zu sparen.

1993 wurde das PT durch eine neue Planungsgröße („new control total“ - NCT) ersetzt. Diese Größe unterschied sich vom PT, indem sie die gesamten Ausgaben der Gemeinden, aber weder die Privatisierungserlöse noch die konjunkturabhängigen Transferzahlungen enthielt. Das NCT sah einen realen Anstieg der Ausgaben um 1,5\% vor, was unter der Annahme des Trendwachstums von $2 \%$ wieder in ähnlicher, aber gemilderter Weise als beim PT, eine Senkung der Ausgabenquote bedeutete.

\section{Zusammenfassung des dritten Kapitels}

Im dritten Kapitel wird die Schuldenpolitik der Konservativen Regierung dargestellt. Die Konservative Politikwende nach 1979 ist nur verständlich vor dem Hintergrund der vorherigen politischen und ökonomischen Entwicklung im Vereinigten Königreich. Deshalb wird nach einem kurzen Überblick der britischen Schuldenentwicklung im zweiten Abschnitt gezeigt, wie das britische Modell der Nachkriegszeit gescheitert ist. Danach wird die wirtschaftspolitische Konzeption der Konservativen Regierung umrissen. Es folgt eine tiefere Analyse der mittelfristigen Finanzstrategie, welche die makroökonomische Politik darstellt. Inbesondere wird gezeigt, wie die mittelfristige Finanzstrategie sowie die begleitende Ausgabenpolitik eine Strategie zum Schuldenabbau beinhaltete. 
Judith Safford - 978-3-631-75199-2

Downloaded from PubFactory at 01/11/2019 07:12:45AM

via free access 


\section{Viertes Kapitel}

\section{EINE ANALYSE DER SCHULDENSENKUNG IM VEREINIGTEN KÖNIGREICH}

In diesem Kapitel wird die tatsächliche Entwicklung der Staatsfinanzen zwischen 1979 und 1994 untersucht. Um einen Vergleich mit der vorherigen Regierung zu ermöglichen und damit das Ausmaß des Politikwechsels zu verdeutlichen, beginnen einige der folgenden Zeitreihen allerdings mit dem Amtsantritt der Labour Regierung im Jahre 1974. Ziel des Kapitels ist es, die Beiträge der jeweiligen Bestandteile der Budgetbeschränkung zum Abbau der Staatsverschuldung zu identifizieren und soweit als möglich zu quantifizieren.

Die Analyse beginnt mit der Ermittlung des Finanzierungsdefizits, welches als Ausgangspunkt der Untersuchung dient. ${ }^{328}$ Danach wird der jeweilige Beitrag von Inflation, realem Wachstum und nominalem Finanzierungsdefizit an der Entwicklung der gesamten Schuldenquote geschätzt. Anschließend wird das Finanzierungsdefizit um die Inflation bereinigt, um zum realen Finanzierungsdefizit zu gelangen. In den folgenden Abschnitten werden dann die einzelnen Komponenten der Budgetbeschränkung isoliert und ihr Beitrag zum Schuldenabbau dargelegt. Zuerst wird der jeweilige Beitrag der drei Finanzierungskomponenten geschätzt. Danach wird der Finanzbedarf nach öffentlichen Betrieben, Gemeinden und Zentralstaat aufgeschlüsselt. Schließlich wird die Entwicklung der Einnahmen und Ausgaben untersucht.

\section{Der öffentliche Kreditbedarf, das reale Finanzierungsdefizit und der Schuldenstand}

\section{1) Die Abgrenzung des Finanzierungsdefizits}

Im vorigen Kapitel wurde gezeigt, wie die Abgrenzung des öffentlichen Kreditbedarfs (PSBR) im Vereinigten Königreich die Privatisierungserlöse als negative Ausgaben oder als ein finanzwirtschaftspolitisches Instrument erfaßt. Der PSBR wurde durch die Gleichung (1) definiert:

${ }^{328}$ Die relevanten Zeitreihen sowie Erläuterungen zu den Berechnungen in diesem Kapitel befinden sich im Anhang. 
(1)

$$
P S B R \equiv P_{t} G_{t}+T r_{t}-T_{t}+i_{t-1} B_{t-1}-\left(A_{t}-A_{t-1}\right)=\left(M_{t}-M_{t-1}\right)+\left(B_{t}-B_{t-1}\right) .
$$

Die Analyse der Finanzierungsquellen hat jedoch gezeigt, daß es sich wegen ihrer finanzpolitischen Äquivalenz mit der Staatsverschuldung empfiehlt, die Privatisierungserlöse als ein Finanzierungsinstrument zu behandeln, d. h. als positiven Beitrag auf der rechten Seite der Gleichung (1) zu rechnen:

$$
P_{t} G_{t}+T_{t}-T_{t}+i_{t-1} B_{t-1}=\left(M_{t}-M_{t-1}\right)+\left(B_{t}-B_{t-1}\right)+\left(A_{t}-A_{t-1}\right)
$$

Unter Berücksichtigung dieser Korrektur und dem Abzug von einigen anderen Finanztransaktionen, ${ }^{329}$ läßt sich das Finanzierungsdefizit ermitteln, das für die weitere Analyse zugrunde gelegt wird. Die beiden Zeitreihen sind in Abbildung 13 dargestellt als Prozentanteile des BIP. Wie zu erwarten, fällt das Finanzierungsdefizit fast durchweg höher als der PSBR aus. Der Beitrag der Privatisierung zur Entlastung der Staatsfinanzen, welcher dem Unterschied zwischen dem PSBR und dem Finanzierungsdefizit entspricht, fällt seit 1984 kontinuierlich positiv aus.

\section{Abbildung 13: PSBR, Finanzierungsdefizit und Schuldenquote}

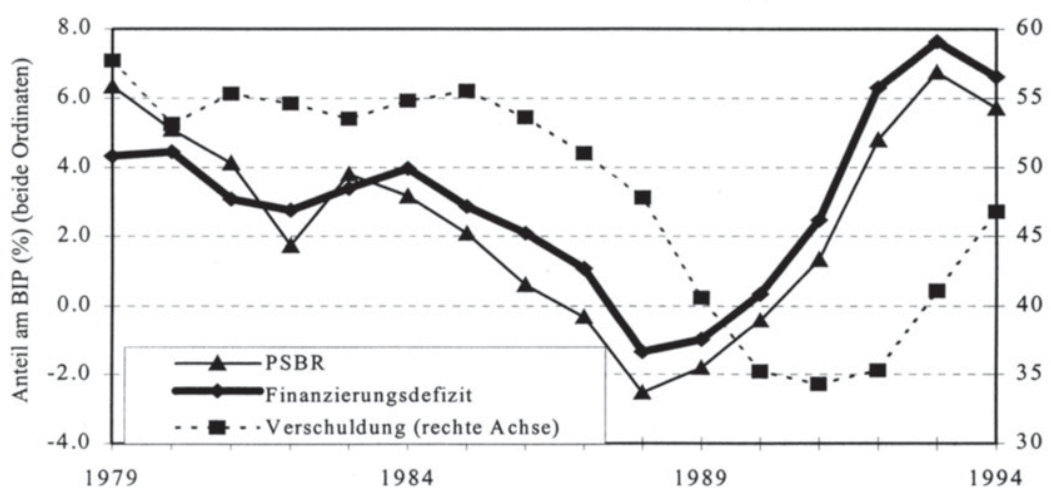

Quelle: CSO Blue Book, BoEQB und eigene Berechnungen

Die Abbildung gibt auch die Brutto-Schuldenquote wieder (vgl. Abbildung $10 \mathrm{im}$ 3. Kapitel, S. 98). Nicht überraschend weisen die Kurven der beiden Finanzierungsströme und diejenige des Schuldenbestandes im Verhältnis zum BIP einen relativ ähnlichen Verlauf auf. Die Budgetüberschüsse zwischen 1987 und 1990, sowie die ansteigende Neuverschuldung Anfang der 80er und 90er Jahre spiegeln

${ }^{329}$ Der öffentliche Kreditbedarf umfaßt neben den Privatisierungseinnahmen verschiedene sonstige Finanztransaktionen, darunter als wichtigste diejenigen für die Pensionskasse des öffentlichen Dienstes und furr periodengerechte Werteberichtigungen (das sogenannte "accruals adjustment"). 
sich, wie zu erwarten wäre, in einer Änderung der Schuldenquote wider. Die Entwicklung in den anderen Defizitjahren deckt sich jedoch nicht mit den jährlichen Änderungen der Schuldenquote. In der Regel geht eine positive Netto-Neuverschuldungsquote mit einer sinkenden oder stabilen Schuldenquote einher. Diese Abweichungen lassen sich teilweise mit Unterschieden im Geltungsbereich und Bewertungsverfahren bei der Berechnung dieser Zeitreihen erklären. ${ }^{330}$ Ein wichtigerer Schritt zur Konsistenz zwischen der Entwicklung von Schuld- und Budgetquote wird jedoch durch die Berücksichtigung der Inflations- und Wachstumswirkungen auf den Schuldenbestand gemacht.

\section{2) Die Auswirkungen von Wachstum, Inflation und Neuverschuldung auf die Schuldenquote}

Es wurde bereits im ersten Kapitel gezeigt, wie die Schuldenquote sich aus dem Schuldenstand im Zähler und dem nominalen Einkommen im Nenner zusammensetzt und folglich von Veränderungen des Schuldenbestandes, des realen Einkommenswachstums und des Preisniveaus bestimmt wird $(b=B / Y P)$. Eine Schätzung der jeweiligen Beiträge dieser drei Komponenten zur Entwicklung der Schuldenquote läßt sich arithmetisch durch die Aufspaltung der Veränderung nach Wirkung der Inflations- und Wachstumsrate auf den Nenner und des Finanzierungsdefizits auf den Zähler ex post ermitteln. Das Ergebnis ist in der Abbildung 14 graphisch dargestellt. Eine Darstellung der Finanzierungskomponenten als Anteil am BIP wurde gewählt, weil dies die jeweiligen Beiträge zur Entwicklung der Schuldenquote sehr anschaulich macht.

${ }^{330}$ Im folgenden wird davon abgesehen, daß sich beispielsweise die Zahlen zur Neuverschuldung auf das Kalenderjahr beziehen, der Schuldenbestand jedoch am 31. März gemessen (am Ende des Finanzjahrs) wird. Deshalb wird die Entwicklung der Neuverschuldung nach dem 31.03. eines gegebenen Jahres erst in der Bestandsänderung im darauffolgenden Jahr erfaßt. Methodische und statistische Gründe erklären weitere Bewertungsunterschiede zwischen der Zeitreihe der Bestandsgröße Staatsschuld und der Stromgröße Budgetdefizit. Für ausführliche Erläuterungen und Berechnung der Korrekturposten (Stock-flow adjusfment) siehe Bank of England (1994), S. 349. 


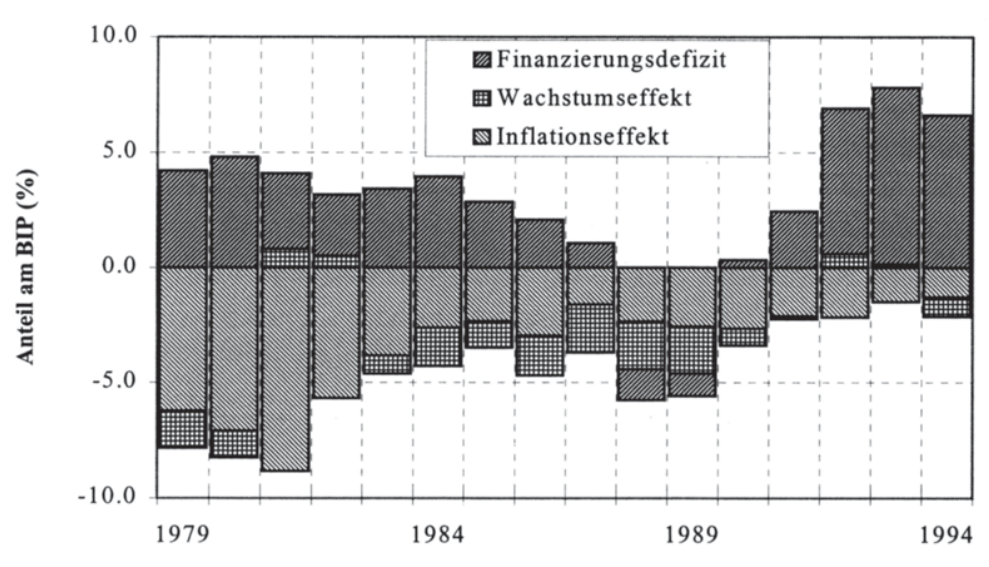

Quellen: BoEQB, CSO Blue Book und eigene Berechnungen

Abbildung 14 veranschaulicht, wie zwischen 1979 und 1991 eine Senkung der Schuldenquote um über $23 \%$ ermöglicht wurde und wie sie anschließend bis 1994 wieder um 13\% anstieg (vgl. hierzu Abbildung 13). In den Rezessionsjahren von 1981-82 und 1992-93 wirkte negatives Wachstum erhöhend auf die Schuldenquote. Finanzierungsüberschüsse in den Jahren 1988 und 1989 trugen zur Senkung der Schuldenquote bei. Ansonsten haben positive Wachstums- und Inflationsraten die Auswirkungen der Finanzierungsdefizite auf die Schuldenquote in der Regel überkompensiert, seltener auch gemildert. Vor allem am Anfang des betrachteten Zeitraums fiel die Inflationswirkung sehr hoch aus. Die niedrigen Inflationsraten ab 1982 bedeuten, daß das gegebene Defizit sich stärker auf den Schuldenstand auswirkte. Dies erklärt die relative Konstanz der Schuldenquote zwischen 1982 und 1985. Ab Mitte der 80er Jahre fielen die Wachstumseffekte stärker ins Gewicht und die Schuldenquote nahm wieder ab. In den 90er Jahren fielen Inflation und Wachstum wesentlich niedriger aus. Somit kamen die höheren Finanzierungsdefizite auf den Schuldenbestand stärker zum Tragen, und die Schuldenquote stieg wieder.

Insgesamt haben zwischen 1979-1994 reale Wachstumseffekte eine Senkung der Schuldenquote von circa $14 \%$ bewirken können. ${ }^{331}$ Der kumulierte Beitrag der Inflation reduzierte die Schuldenquote real um $56 \%$ des BIP. Die Neuverschuldung sorgte dagegen für einen kumulierten Anstieg der Schuldenquote von ungefähr

331 Das Wirtschaftswachstum hat auch im Konjunkturverlauf starke Auswirkungen auf die Budgeteinnahmen und -ausgaben, welche jedoch bei dieser Berechnung nicht direkt ausgewiesen werden können. 
$50 \% .{ }^{332}$ Mit anderen Worten, zwischen 1979 und 1994 wurde der Wert der Neuverschuldung durch die Inflation komplett vernichtet.

\section{3) Inflationsbereinigung des Finanzierungsdefizits}

Die obigen Berechnungen schätzten die Auswirkungen der Inflation auf den Nenner der Schuldenquote. Eine andere Methode, die Inflationswirkungen zu erfassen, berechnet den Tilgungseffekt der Inflation auf das Budgetdefizit, welcher infolge der Inflation zwischen zwei Perioden auftritt (vgl. die theoretischen Ausführungen im 2. Kapitel, Abschnitt II.2). Die Abbildung 15 zeigt die Entwicklung des Finanzierungsdefizits, sowie das Defizit nach Bereinigung dieses Inflationseffekts.

\section{Abbildung 15: Inflationsbereinigung des Finanzierungsdefizits}

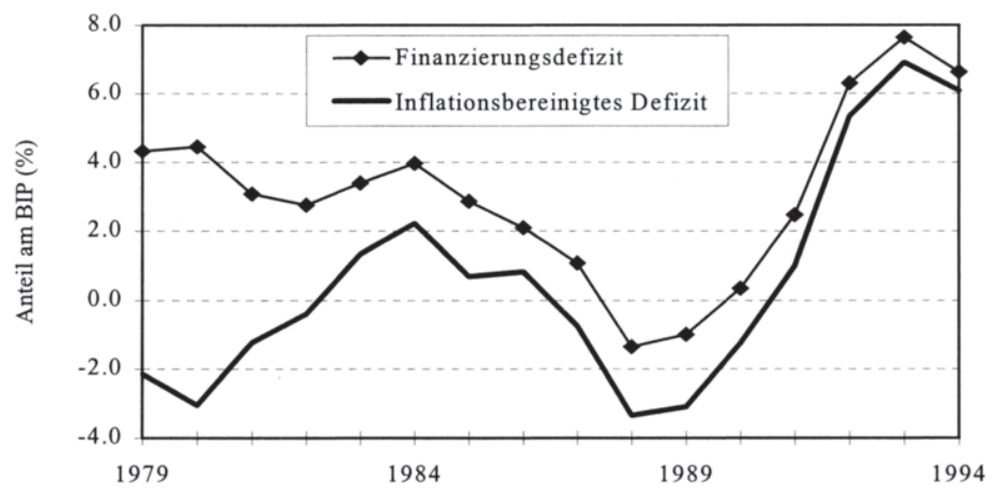

Quellen: Abbildung 13, BoEQB, CSO und eigene Berechnungen

Nach Berücksichtigung der Inflation verwandelt sich das Budgetdefizit häufig in einen Überschuß, was konsistent ist mit einer sinkenden Staatsverschuldung (vgl. Abbildung 13). Während ein Finanzierungsüberschuß erst 1988 erreicht wurde, war das inflationsbereinigte Defizit zwischen 1979 und 1982 positiv, sowie erneut bereits wieder ab 1987. Ab 1991 sind die inflationsbereinigten Staatsfinanzen abermals defizitär. Es fällt auf, daß die zwei Kurven sich im Zeitverlauf nähern, weil fallende Inflationsraten die Inflationstilgung reduzieren.

332 Ungeklärt für die Entwicklung der Schuldenquote bleibt ein Fehlbetrag von 4\% über der ganzen Periode (vergleiche die berechnete und die tatsächliche Verschuldungsquote in Abbildung 31 des III. Anhangs) . 


\section{4) Auswirkungen der Inflation auf die Steuereinnahmen ${ }^{333}$}

Im zweiten Kapitel wurde gezeigt, wie sich die Inflation auf die Steuereinnahmen auswirkt. Dabei wurde der Effekt der kalten Progression und die Auswirkungen der Nominalwertbesteuerung von Zinseinkünften vorgestellt. Der erste Effekt dürfte im Vereinigten Königreich relativ unbedeutend sein. Das „Rooker-Wise Amendment“ (1977) des Finanzgesetzes sieht die periodische Inflationsanpassung der Steuertarife vor. Nur in speziellen Fällen, die eines gesonderten Gesetzes bedürfen, kann diese Anpassung verzögert werden. Die Konservative Regierung machte drei Mal von dieser Ausnahmeregel Gebrauch: 1981 wurde der Einkommensteuertarif der Inflation von $11,5 \%$ nicht angepaßt, ${ }^{334}$ ebenso 1993 und 1994 bei Inflationsraten von jeweils 3,3\% und 2,1\%. Diese Anpassungen wurden in den folgenden Jahren nachgeholt. Insgesamt sanken im Rahmen der Steuerreform die Steuertarife real. Aus diesem Grund fielen die Gewinne aus der kalten Progression im Vereinigten Königreich vermutlich nicht sehr hoch aus und werden hier vernachlässigt. ${ }^{335}$

Die Gewinne aus der Besteuerung der Zinszahlungen auf die Staatsschuld nach dem Nominalwertprinzip brachten vermutlich höhere inflationsbedingte Einnahmen. Eine genaue Berechnung ist jedoch nicht möglich, da weder der Realzins noch der effektive Steuersatz auf die Zinserträge bekannt sind. Die nominale Zinslast kann jedoch in eine Realzinskomponente von schätzungsweise $2,5 \%$ und eine Inflationsrate von $7 \%$ aufgeteilt werden. ${ }^{336}$ Daraus läßt sich die Inflationskomponente bei den nominalen Zinszahlungen berechnen. Nach dem Nominalwertprinzip werden Kapitalerträge im Vereinigten Königreich zu einem Grenzsteuersatz von $25 \%$ besteuert. Unter der Annahme, daß die durchschnittliche Besteuerung bei $15 \%$ liegt, ${ }^{337}$ erzielt der Staat infolge der Inflation Steuereinkünfte in Höhe von $15 \%$ der Inflationskomponente. Entsprechend dieser Rechnung führte die Besteuerung der nominalen Zinsen auf der Staatsschuld zwischen 1979 und 1994 zu einer jährlichen Budgetentlastung von durchschnittlich etwa $0,45 \%$ am BIP.

\footnotetext{
${ }^{333}$ Die Inflationsauswirkungen auf die Staatsausgaben tragen nicht zur Konsolidierung der Staatsfinanzen bei (2. Kapitel, Abschnitt II.3).

334 Was praktisch einer Anhebung des Eingangssteuersatzes um 3\% gleichkam, Shaw (1983), S. 331.

335 Giles und Johnson (1994), S. 69ff.

${ }^{336}$ Die durchschnittliche Inflationsrate zwischen 1979 und 1994 lag bei 7\%. Entsprechend den Bräuchen der Bank of England wird ein durchschnittlicher Realzins von 2,5\% angenommen, Kennedy (1989), S. 246.
}

337 Ähnlich verfahren Buiter und Miller (1983), S. 328. 


\section{Die Zusammensetzung des Finanzierungsdefizits und ihre institutionelle Aufteilung}

Nachdem die jeweiligen Beiträge des Wachstums und der Inflation zum Schuldenabbau ermittelt worden sind, widmet sich die Analyse nun der Zusammensetzung des Finanzierungsdefizits. Zunächst werden die jeweiligen Finanzierungsbeiträge des staatlichen Budgets aus den Einnahmen der Privatisierung, der Geldfinanzierung und der Schuldfinanzierung aufgeteilt. Anschließend wird das Finanzierungsdefizit auf die drei öffentlichen Schuldner aufgeteilt.

\section{1) Die Finanzierung des staatlichen Budgets}

Die Finanzierungsquellen des Finanzierungsdefizits sind in der Abbildung 16 dargestellt. Die Finanzierungskomponenten werden auch hier als Anteil am BIP veranschaulicht, weil so die jeweiligen Beiträge zur Senkung der Defizitquote leicht zugeordnet werden können.

\section{Abbildung 16: Quellen der Defizitfinanzierung im Vereinigten Königreich}

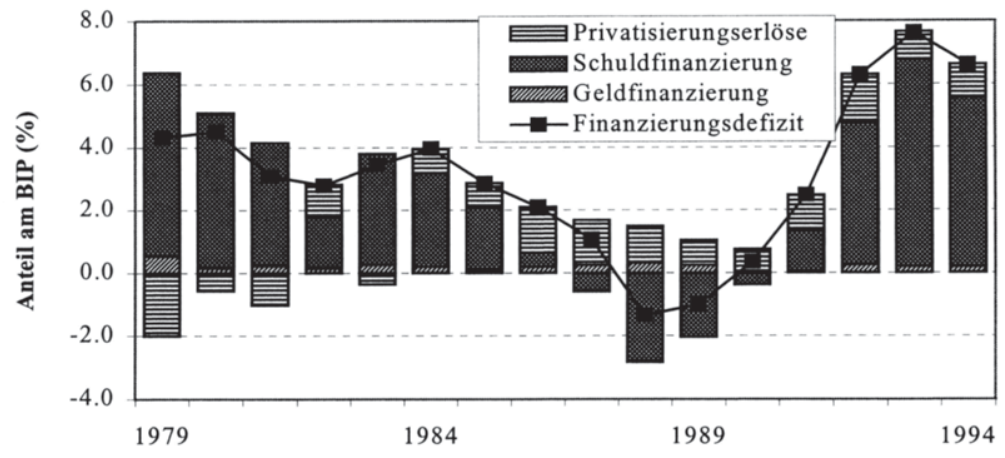

Quelle: CSO Blue Book, eigene Berechnungen

\section{a) Einnahmen aus der Geldemission}

Die Geldfinanzierung oder der Seignorage, wie sie im zweiten Kapitel abgeleitet wurde, spielt eine sehr geringe Rolle bei der Budgetfinanzierung im Vereinigten Königreich. Unter der Labour-Regierung zwischen 1974 und 1978 lag sie im 
Durchschnitt bei $0,73 \%$ des BIP. Der Anteil der Geldfinanzierung sank noch weiter nach 1979 und blieb relativ konstant bis 1994 mit durchschnittlich $0,22 \%$ des BIP. ${ }^{338}$ Dieser geringe Anteil ist auch konsistent mit den Inflationszielen der MTFS.

\section{b) Finanzierung aus der Privatisierungspolitik}

Eine wesentlich größere Rolle spielen dagegen die Privatisierungserlöse, wobei sie erst seit 1984 signifikant zur Budgetfinanzierung beitragen, mit einem Anteil am BIP von circa $0,5 \%$ bis $1,5 \%$. Im ersten Haushaltsplan 1979 waren Einnahmen in Höhe von lediglich $£ 1 \mathrm{Mrd}$. vorgesehen, im wesentlichen durch einen Teilverkauf von British Petroleum (BP). In den drei Folgejahren beschränkten sich die Verkäufe auf weitere Teile des Ölunternehmens, der Ölvorräte und der Ölforschungsrechte. Nach 1983 nahmen die Einnahmen aus der Privatisierungspolitik zu. Die staatlichen Unternehmen für Gas, Elektrizität, Wasser und Telekommunikationen wurden veräußert. Außerdem wurden Häfen, Flug-, Bus- und Schiffsunternehmen, sowie die Firmen Jaguar, British Aerospace, und Cable und Wireless wieder privatisiert. ${ }^{339}$ Bis Ende 1994 verzeichnete die Regierung Nettoeinnahmen von ungefähr $£ 60 \mathrm{Mrd} .{ }^{340}$ Ende der $80 \mathrm{er}$ Jahre reichten die Beiträge aus, um fast das gesamte Budgetdefizit abzudecken.

\section{c) Der Anteil der Schuldfinanzierung}

Die Schuldfinanzierung macht in der Regel mit durchschnittlichen $75 \%$ den größten Finanzierungsanteil aus (durchschnittlich 2,3\% des BIP). 1994 teilte sie sich vorwiegend in langfristige Staatspapiere (circa 80\%) und nicht-börsengängige Sparbriefe (circa $20 \%$ ) auf. ${ }^{341}$ Derzeit sind circa $15 \%$ der britischen Staatsschuld als indexierte Schuldpapiere emittiert und die Regierung ist bemüht, diesen Anteil zu erhöhen. ${ }^{342}$ Die Abbildung 16 zeigt, wie ab 1983 die Schuldenausgabe als Anteil am BIP bis 1987 abnimmt. 1986 war die Finanzierung aus Privatisierungserlösen höher als die Schuldenausgabe. Zwischen 1987 und 1990 ist die Staatsverschul-

${ }^{338}$ Cukierman et al. schätzen die Seignorage ebenfalls als gering ein: Zwischen 1975 und 1982 circa 0,55\% am BIP, S. 50. Nach Buiter (1985), S. 25 lagen die Einnahmen aus der Seignorage bei durchschnittlich 0,55\% sogar über dem Zeitraum 1948 bis 1983.

${ }^{339}$ Eine Zusammenstellung der wichtigsten Privatisierungen findet sich im Anhang.

${ }^{340}$ Djaroueh (1994), S. 80 und CSO Financial Statistics. Diese Zahl unterschätzt die budgetwirksamen Einnahmen etwas, weil manche Privatisierungserlöse an die Industrien selbst bezahlt und dann durch Anpassung ihrer Kreditaufnahme kompensiert wurden.

${ }^{341}$ Bank of England (1994), S. 350, und eigene Berechnungen.

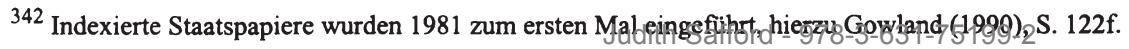


dung sogar negativ gewesen. Zwischen Oktober 1988 und Januar 1991 wurden keine langfristigen Staatspapiere herausgegeben. ${ }^{343}$ Danach steigt die Schuldausgabe allerdings wieder sehr stark an.

\section{2) Die Aufteilung der Verschuldung zwischen den öffentlichen Schuldnern}

Die Finanzen der öffentlichen Hand im Vereinigten Königreich sind in drei Bereiche gegliedert: Den Zentralstaat, die Gemeinden und die verstaatlichten Industriebetriebe. Eine interessante Entwicklung bei der Schuldenaufnahme wird sichtbar, wenn entsprechend dieser Aufteilung die jeweiligen Finanzierungsdefizite errechnet werden, wie die Abbildung 17 veranschaulicht.

\section{Abbildung 17: Die Finanzierungsdefizite der öffentlichen Institutionen}

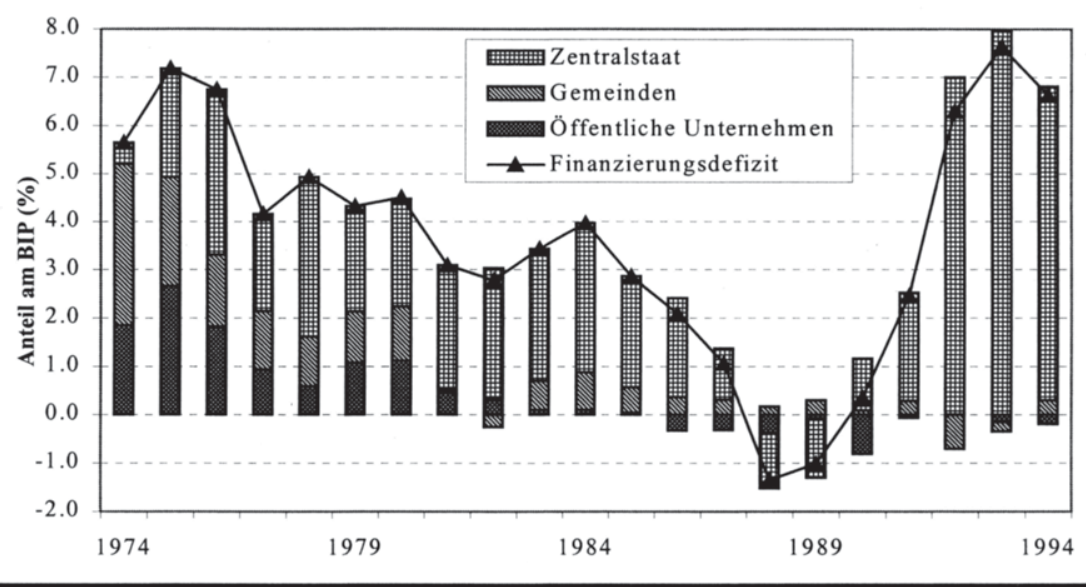

Quelle: CSO Blue Book, eigene Berechnungen

Es fällt sofort auf, daß sich die Verteilung der Neuverschuldung auf die öffentlichen Institutionen in den letzten zwanzig Jahren verschoben hat. Am Anfang der Periode war das Schuldverhalten der drei Bereiche relativ gleichmäßig verteilt. Von 1974 bis 1979 wurden die Defizite durchschnittlich zu 40\% vom Zentralstaat verursacht. Die Gemeinden hatten einen Anteil von $33 \%$ und die öffentlichen Unternehmen von $27 \%$. Schon in den ersten fün Jahren (1979 bis 1984) der Konservativen Regierung fiel der Anteil der Neuverschuldung des Zentralstaats mit 69\% wesentlich höher aus. Ab der zweiten Hälfte des Jahrzehnts (nach 1986) erwirt- 
schafteten die öffentlichen Unternehmen dann Überschüsse, welche den zentralstaatlichen Defizitanteil mitfinanzierten. Von 1989 bis einschließlich 1994 konnten die Nettoüberschüsse der anderen zwei Sektoren die zentralstaatliche Schuldaufnahme um durchschnittlich $5 \%$ entlasten.

Diese Zentralisierungstendenz hängt mit den wesentlichen Reformen bei der Finanzierung von öffentlichen Institutionen der Konservativen Regierungen seit 1979 zusammen. Erstens wurde während der Vorbereitungen zur Privatisierung die Kreditaufnahme der öffentlichen Unternehmen massiv reduziert. Dies geschah durch Vermögensveräußerung, starke Preiserhöhungen ihrer Produkte sowie Rationalisierungsmaßnahmen, wie beispielsweise Personalabbau. Im Verlauf der 80er Jahre wurden infolge der Privatisierung die öffentlichen Unternehmen dann auch zunehmend aus dem öffentlichen Budget ausgegliedert. Zweitens wurden die Kontrollbefugnisse der Zentralregierung über die Schuldfinanzierung der Gemeinden und der übrigen öffentlichen Unternehmen ausgeweitet, einschließlich der Festsetzung von effektiven Höchstgrenzen der Schuldaufnahme bzw. Auflagen zur Schuldentilgung durch Vermögensveräußerung. ${ }^{344}$ Insgesamt hat sich der Zentralstaat seit 1979 eine weitreichende Kontrolle über die strukturellen, gesetzlichen und finanziellen Rahmenbedingungen aller öffentlichen Institutionen gesichert. ${ }^{345}$

In der weiteren Analyse der Budgetrestriktion wird auf die Finanzen der öffentlichen Unternehmen aufgrund ihrer relativ geringen Bedeutung nicht weiter eingegangen. Somit bezieht sich die weitere Analyse der Schuldenentstehung auf die Neuverschuldung der Zentralregierung und der Gemeinden.

\section{Die budgetpolitischen Faktoren der Schuldensenkung}

Bis hierhin wurde gezeigt, wie das Wirtschaftswachstum und die Inflation die reale Last der Budgetfinanzierung mindert und wie der Seignorage und die Privatisierung den schuldenfinanzierten Anteil des Finanzierungsdefizits verringert. Die Regierung hat aber auch aktive budgetpolitische Maßnahmen zur Schuldensenkung ergriffen, die im folgenden zusammen mit der resultierenden Entwicklung der Einnahmen und Ausgaben untersucht werden. Die Auswirkungen der verfolgten Politik werden mit den Ergebnissen des zweiten Kapitel über die Strategien zur Schuldensenkung verglichen.

344 Ausführlicher hierzu Safford (1994), S. 431f.

${ }^{345}$ Glynn (1987), S. 69f., auch Humes IV, (1991), S. 109f, sowie Economist, 8.08.92, S. 26. 


\section{1) Der Primärsaldo}

Der nominale Primärsaldo $\left(P_{t} G_{t}+T_{t}-T_{t}\right)$ zeigt den Deckungsgrad der Steuern und sonstiger Einnahmen gegenüber den realen Ausgaben und den Transferzahlungen. Er mißt wie aktuelle Politikmaßnahmen die öffentliche Netto-Verschuldung verbessern oder verschlechtern. Er hängt zum einen von der Einnahmen- und Ausgabenpolitik der Regierung ab, zum anderen wird er stark von konjunkturellen Einflüssen geprägt, da die Einkommensentwicklung die Höhe der Ausgaben, insbesondere der Transferzahlungen, sowie der Steuereinnahmen beeinflußt. Diesen Unterschied zwischen beabsichtigter Gestaltung und ungeplanten Schwankungen der Einnahmen und Ausgaben im Konjunkturzyklus versucht man oft durch die Aufteilung des Defizits auf einen sogenannt strukturellen und konjunkturellen Teil zu erfassen. Das strukturelle Defizit mißt die Wirkung von aktiven Politikmaßnahmen im Sinne der nachfrageorientierten Steuerung und wird von nationalen Institutionen, aber auch von supranationalen Organisationen wie z. B. der EUKommission, dem IWF und der OECD ermittelt. ${ }^{346}$

Auf eine derartige Aufteilung wird jedoch in dieser Arbeit verzichtet. Zum einen ist die Berechnung des strukturellen Defizits konzeptionell sehr schwierig. Unterschiedliche Modellannahmen führen zu beträchtlichen Divergenzen, wie die teilweise sehr unterschiedlichen Ergebnisse der obengenannten Stellen bezeugen. Somit sind Aussagen über das strukturelle Defizit mit einer gewissen Willkür behaftet. Zum anderen hat sich die Konservative Regierung seit 1979 bewußt von der Nachfragesteuerung abgewendet. Ihre mittelfristige Finanzierungsstrategie verfolgte sie unabhängig von der wirtschaftlichen Entwicklung. Somit stellte die Konjunkturentwicklung zwar eine Rahmenbedingung für den Schuldenabbau dar, sollte aber weder einen Einfluß auf die verfolgte Politik haben, ${ }^{347}$ noch ein Kriterium für ihren Erfolg sein. Deswegen wird im folgenden nur kurz auf die konjunkturelle Entwicklung in der Untersuchungsperiode eingegangen.

${ }^{346}$ Barrell et al. (1994) geben einen Überblick der verschiedenen Ansätze der supranationalen Institutionen (IWF, OECD und EC).

347 Seit 1993 räumt die Regierung jedoch ein, daß das Budgetdefizit im Konjunkturverlauf schwanken dürfte. Siehe 3. Kapitel, Abschnitt III.3. 


\section{Abbildung 18: Der Primărsaldo 1979 bis 1994}

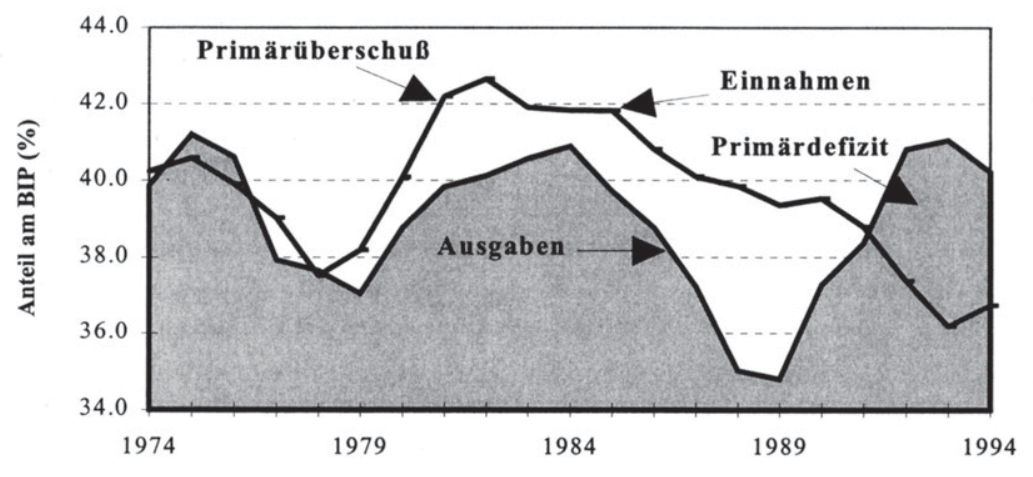

Quelle: CSO Blue Book, eigene Berechnungen

Abbildung 18 zeigt die Entwicklung des Primärdefizits. Die Ausgaben sind als schattierter Bereich und die Einnahmen als Linie eingezeichnet. Der Primärsaldo stellt folglich den Unterschied zwischen diesen zwei Kurven dar.

Das expansive Ausgabeverhalten der Labour Regierung 1974 und 1975 läßt sich am damaligen Primärdefizit und an der hohen Ausgabenquote ablesen. Nach Einführung des IWF-Anpassungsprogramms 1976 sank die Ausgabenquote sehr rasch und der Saldo wandelte sich wieder in einen Überschuß um. 1978 verschlechterten sich aber die Staatsfinanzen erneut und der Primärsaldo befand sich wieder knapp im Defizit.

Die Konservative Partei kam im Mai 1979 an die Macht. In diesem Monat befand sich die Wirtschaft auf dem Höhepunkt eines Konjunkturzyklus. ${ }^{348}$ Der folgende Abschwung erreichte seinen Tiefpunkt im Januar 1981. Entsprechend den Vorstellungen der MTFS wurde die konjunkturelle Entwicklung bei der Gestaltung der Fiskalpolitik nicht berücksichtigt. Die Ausgaben wurden in der Rezession nicht antizyklisch erhöht, weswegen ihr Anstieg verhältnismäßig bescheiden ausfiel. $\mathrm{Zu}$ sammen mit dem gleichzeitigen Anstieg der Einnahmenquote führte dies während der gesamten Rezession 1980-81 zur Erhaltung eines Überschusses. Erst im Laufe des wirtschaftlichen Aufschwungs 1982 nahm die Einnahmenquote prozyklisch ab. Ein kleiner wirtschaftlicher Abschwung im Frühjahr 1984 dauerte nur einige Monate. Er hing mit dem jahrelangen Bergarbeiterstreik zusammen, der im März 1984

348 Die Indikatoren für den Konjunkturzyklus stammen vom britischen statistischen Bundesamt, CSO, Economic Trends. 
anfing und das Wirtschaftswachstum 1984 etwas drosselte. Die Entwicklung der Einnahmen flachte sich in dieser Zeit bis $1985 \mathrm{ab}$. Danach nahm sie unabhängig vom Konjunkturzyklus bis 1993 praktisch ununterbrochen ab.

Von 1979 bis 1984 nahmen die Ausgaben zu. Danach nahmen sie rapide ab und ermöglichten trotz sinkender Einnahmen bis 1989 immer größer werdende Überschüsse. In der nächsten Rezession 1990-92 stiegen die Ausgaben aber sehr schnell an. Aufgrund gleichzeitig stark fallender Einnahmen geriet 1991 der Primärsaldo zum ersten Mal seit 1978 ins Defizit.

Bei diesem ersten Überblick wird der Beitrag der Primärüberschüsse zum Schuldenabbau der $80 \mathrm{er}$ Jahre offenbar. ${ }^{349}$ Auch wird die prozyklische Wirkung der MTFS bis 1990 deutlich. Während der Rezession 1980-82 stieg die Einnahmenquote um 4,5\% des BIP 350 und im langen Aufschwung bis 1989 wurde sie um 3,3\% gesenkt. Danach beschleunigte sich die Einnahmensenkung mit einer weiteren $\mathrm{Ab}$ nahme um 3,2\% bis 1993. Die Ausgabenentwicklung entspricht eher dem antizyklischen Muster. Der Anstieg um 2,8 \% in der Rezession fiel jedoch relativ gering aus und setzte sich während des Aufschwungs bis 1984 fort. Danach sind die Ausgaben bis 1989 von $40,9 \%$ auf $34,8 \%$ stark gesunken. Nach dem zyklischen Höhepunkt im August 1988 stiegen in der Rezession 1990-92 und danach bis 1993 die Ausgaben erneut sehr rapide bis auf $41 \%$ an. Bei weiter sinkender Einnahmenquote entstand ein beträchtliches Primärdefizit, das auch im starken Aufschwung ab Mitte 1992 erhalten blieb.

Nach diesem Überblick des Primärsaldos und dessen Beitrag zur Konsolidierung gibt eine nähere Analyse der Einnahmen und Ausgaben Auskunft, wie Politikmaßnahmen seine Entwicklung beeinflußt haben.

\section{2) Der Beitrag der Einnahmen}

Nach einem Überblick über die gesamten Einnahmen wird die Entwicklung der einzelnen Steuereinnahmen vor dem Hintergrund der Konservativen Steuerpolitik näher betrachtet.

\footnotetext{
${ }^{349}$ Im Durchschnitt betrug der Primärüberschuß 2,4\% des BIP von 1979 bis 1990.

${ }^{350}$ Wenn nicht anders vermerkt, beziehen sich die Prozentangaben im folgenden immer auf den Anteil der Meßgröße am BIP. 


\section{a) Die Entwicklung der gesamten Einnahmen}

Die Einnahmen setzen sich aus den Steuern, den Zinseinkünften sowie den sonstigen Einkünften zusammen. Ihre Entwicklung wird in der Abbildung 19 veranschaulicht. Die Einnahmenquote im Vereinigten Königreich lag in den letzten zwanzig Jahren knapp unter 40\%, mit leicht steigender Tendenz. 1994 liegt sie im internationalen Vergleich deutlich unter dem Durchschnitt der OECD Länder. ${ }^{351}$

\section{Abbildung 19: Die Entwicklung der Staatseinnahmen 1974-1994}

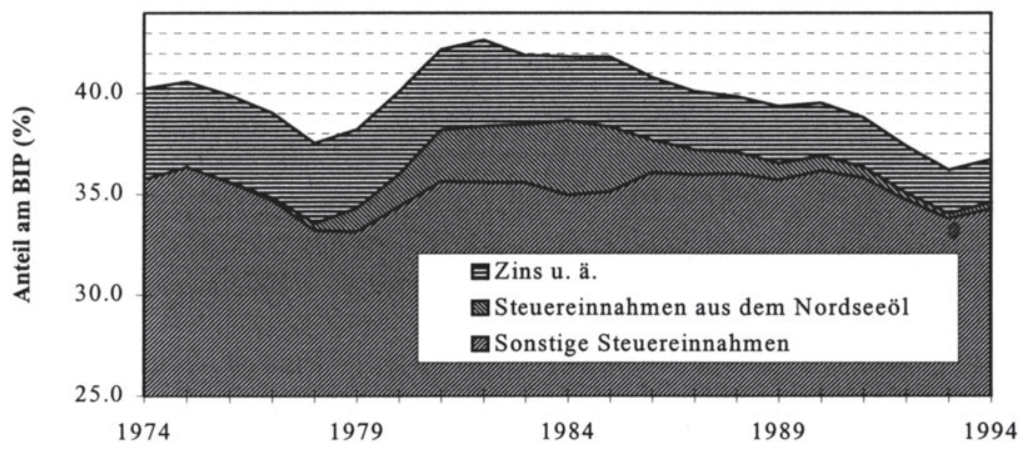

Quelle: CSO Blue Book, Bean (1987), eigene Berechnungen

Die Abbildung zeigt, wie die Steuereinnahmen zwischen zwei Tiefpunkten (1978: 33,6\% und 1993: 34,0\%) auf 38,7\% im Jahre 1984 stiegen. Drei Entwicklungen bei den britischen Staatseinnahmen werden aus der Abbildung deutlich.

Erstens läßt sich die Zunahme der Einnahmen im wesentlichen auf die vorübergehenden Steuereinnahmen und Beteiligungen am Nordseeöl zurückführen. Diese stiegen von $£ 0,56$ Mrd. 1979 - dem Jahr der Konservativen Machtübernahme - auf ihr Maxima von $£ 12$ Mrd. 1984, wo sie 8,8\% der Einnahmen ausmachten (oder $3,7 \%$ am BIP). Ab 1986/87 fielen sie etwas geringer aus, weil der Ölpreissturz sich negativ auf die Einnahmen aus der Nordseeölsteuer auswirkte. ${ }^{352}$ Ende der $80 \mathrm{er}$ Jahre gingen die ausbeutbaren Ölreserven dann langsam zu Ende. Im Jahre 1994 lagen die Einnahmen bei circa $£ 2,2$ Mrd. (0,3\% am BIP) und sind damit wieder unbedeutend geworden. Von 1979 bis 1990 trugen sie im Durchschnitt 4,5\% zu den gesamten Einnahmen bei.

${ }^{351}$ Der OECD-Durchschnitt liegt bei fast 45\%. Hills (1996), S. 73.

352 Budd (1988), S. 2 f. 
Zweitens sind die anderen Steuereinnahmen bereinigt um die Einnahmen aus der Nordseeölproduktion relativ konstant geblieben. Ihr Tiefpunkt war 1979, als die Konservative Partei zur Macht kam. Damals lag die Steuerquote bei 33,2\%. 1990 erreichte sie mit 36,7\% ihr Maximum. Sie variierten somit in der ganzen Perioden lediglich um 3,5\%. Trotz Tarifsenkungen bei den direkten Steuern mit der Absicht die Steuerlast zu senken, ist sie - auch abzüglich der Nordseeöleinnahmen - unter der Konservativen Regierung leicht angestiegen.

Drittens ist der Zinsanteil und ähnlicher Einkünfte stetig von 3,9\% (1979) auf 2,2\% (1993) gesunken. Diese Posten umfassen Einnahmen aus Zins und Dividenden der öffentlichen Unternehmungen und den zugeschriebenen jährlichen Wertzuwachs des öffentlichen Vermögens. Offensichtlich ist diese Entwicklung größtenteils durch die Privatisierungspolitik zu erklären. Die Veräußerung öffentlicher Betriebe bedeutet den Verzicht auf zukünftige Zinseinkünfte und Dividenden und mindert auch die Abschreibungsmöglichkeiten auf öffentliches Vermögen. Ein Teil der reduzierten Abschreibung ist jedoch die Folge einer Senkung im Wert des öffentlichen Vermögenbestandes, die mit niedrigen öffentlichen Investitionen zusammenhängt. ${ }^{353}$

\section{b) Steuerreform und Steuereinnahmen ${ }^{354}$}

Die Steuerpolitik der Konservativen Regierung war vom Glauben an die negativen Anreizeffekte der direkten Besteuerung und vom Wunsch, die Steuerquote zu senken, bestimmt. Bereits 1979 im ersten Haushaltsplan setzte die Regierung den Höchstsatz der Einkommensteuer von $83 \%$ auf $60 \%$, sowie den Normalsatz von $33 \%$ auf $30 \%$ herab. Wie die Analyse der erweiterten Laffer-Kurve im zweiten Kapitel, Abschnitt III.1 zeigte, hängen die Auswirkungen dieser Maßnahmen auf das Steueraufkommen von der Ausgangsposition auf der Laffer-Kurve ab. Die Regierung erwartete von dieser Reform keinen Anstieg der Steuereinnahmen, sondern eine Einnahmenlücke von $£ 4,5$ Mrd. (2,3\% des BIP) im folgenden Jahr, welche nicht durch Privatisierungseinnahmen oder Ausgabensenkungen ausgeglichen werden konnte und das Defizitziel der MTFS gefährdet hätte. ${ }^{355}$ Deshalb erhöhte sie als Kompensationsmaßnahme die indirekten Steuern. Der Standardsatz der Mehrwertsteuer wurde von $8 \%$ auf $15 \%$ angehoben. ${ }^{356}$ Darüber hinaus wurde auch die

\footnotetext{
353 Hills (1996), S. 75.
}

${ }^{354}$ Siehe hierzu insbesondere Keegan (1984), S. 118f, Walters (1986), S. 76ff. und Cairncross (1992), S. 236ff.

355 Vgl. hierzu die Regierungsberichte über das jährliche Finanzgesetz und seine erwarteten Auswirkungen auf das Steueraufkommen im CSO, Economic Trends, November Ausgabe (bis 1992 März Ausgabe).

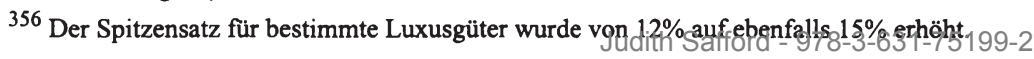


Mineralölsteuer stark hinaufgesetzt. Dazu kam 1980 eine Anhebung der Sozialabgaben und die Einführung einer neuen Steuer auf die Erdöl- und Erdgasförderung.

Im nächsten Jahr befand sich die britische Wirtschaft im Tal einer tiefen Rezession. Konjunkturbedingte Steuerausfälle machten weitere Steuererhöhungen notwendig. Die Regierung erhöhte die direkten Steuersätze nicht. ${ }^{357}$ Statt dessen wurden verschiedene indirekte Steuern, insbesondere die Ölförderungssteuern, die Kraftfahrzeugsteuer sowie die Verbrauchsteuern auf Kraftstoffe, alkoholische Getränke und Tabakwaren erhöht und eine einmalige Sondersteuer auf Bankeinlagen eingeführt. Im Mai 1981 begann ein Aufschwung, der bis 1989 dauerte. In dieser Zeit nahm die Regierung mehrere Senkungen der Einkommensteuer vor. Der Normalsatz wurde schrittweise auf $25 \%$ und der Spitzensatz auf $40 \%$ reduziert und die Freibeträge real erhöht. Von 1982 bis 1989 wurden mit Ausnahme von 1984 im Haushaltsplan jährlich Einkommensteuersenkungen bekanntgegeben. Die erwarteten Einnahmeverluste wurden in den meisten Jahren mit Erhöhungen der Verbrauchssteuern kompensiert.

1990 fiel die Wirtschaft erneut in eine schwere Rezession. Zugleich waren die Ölreserven Großbritanniens weitgehend erschöpft. Beide Entwicklungen hatten große Steuerausfälle zur Folge. Wesentliche Einnahmenerhöhungen wurden jedoch zunächst nicht vorgenommen. ${ }^{358}$

Im gleichen Jahr nahm die Regierung eine umfassende Reform der Gemeindefinanzen vor, die vor allem die Einführung der ,poll tax“ beinhaltete (hierzu 2. Kapitel, Abschnitt III.2). Die Einnahmen von dieser Steuer waren viel niedriger und die Erhebungskosten viel höher als erwartet. ${ }^{359}$ Eine Erhöhung des Normalsatzes der Mehrwertsteuer im Jahre 1991 von $15 \%$ auf 17,5\% sollte die Einnahmenausfälle ersetzen.

Trotz der rapiden Verschlechterung der öffentlichen Finanzen infolge der vergangenen Rezession wurden Maßnahmen zur Erhöhung der Steuereinnahmen erst nach der Wiederwahl der Konservativen Regierung im April 1992 beschlossen. Ein niedriger Eingangssatz der Einkommensteuer von 20\% wurde sogar kurz vor der Wahl eingeführt. Nach der Wahl wurden 1993 die größten Steuererhöhungen seit 1981 verabschiedet. ${ }^{360}$ Die Freibeträge auf die Einkommensteuer wurden eingefroren, für Verheiratete sogar gesenkt und die Sozialabgaben für Arbeitnehmer um 1\%

357 Obwohl der unveränderte Einkommensteuertarif bei einer Inflationsrate von $15 \%$ für zusätzliche Einnahmen aus der kalten Progression sorgte.

${ }^{358}$ Es wurde sogar ein Grenzsteuersatz von $20 \%$ für niedriges Einkommen eingeführt. Zur neuen Steuerpolitik, siehe Giles und Johnson (1994), S. $65 \mathrm{ff}$.

${ }^{359}$ Smith (1994), S. 4.

${ }^{360}$ Hierzu Giles und Ridge (1993). 
erhöht. Die steuerliche Absetzung von Hypothekarschuldzinsen wurde stark eingeschränkt. Schließlich wurden die Steuern auf Mineralbrennstoffe und Tabak erhöht. Die bedeutendste der geplanten Reformen dabei war, Brennstoffe für den privaten Haushaltsverbrauch dem Normalsatz der Mehrwertsteuer zu unterwerfen. Diese Veränderung stieß auf großen Widerstand in der Bevölkerung, und das entsprechende Finanzgesetz wurde vom Parlament nur mit einem ermäßigten Steuersatz von $8 \%$ angenommen (vgl. auch 2. Kapitel, Abschnitt III.2).

Die wesentlichen Steuerreformen zwischen 1979 und 1994 sind in der folgenden Tabelle zusammengefaßt:

Tabelle 5: Die wichtigsten britischen Steuersätze

\begin{tabular}{|l|c|c|}
\hline & 1979 & 1994 \\
\hline Spitzensatz der Einkommensteuer & $83 \%$ & $40 \%$ \\
\hline Normalsatz der Einkommensteuer & $33 \%$ & $25 \%$ \\
\hline Eingangssatz der Einkommensteuer & $25 \%$ & $20 \%$ \\
\hline Sozialversicherungsbeiträge (Arbeitgeber) & $10 \%$ & $10,4 \%$ \\
\hline Sozialversicherungsbeiträge (Arbeitnehmer) & $6,5 \%$ & $10 \%$ \\
\hline Körperschaftssteuer & $52 \%$ & $34 \% *$ \\
\hline Normalsatz der Mehrwertsteuer & $8 \%$ & $17,5 \%$ \\
\hline * Für kleine Unternehmen jeweils von 42\% (1979) auf 25\% (1994). & \\
\hline
\end{tabular}

Quellen: Byatt (1989), Giles und Johnson (1994), Shaw (1994)

Insgesamt verfolgten die steuerpolitischen Maßnahmen der Konservativen Regierung ein strukturelles Ziel, nämlich die negativen Anreizeffekte der direkten Besteuerung zu senken. Entsprechend dieses Ziels wurde die Last der direkten Besteuerung vor allem für die oberen Einkommensschichten gesenkt. ${ }^{361}$ Die indirekten Steuern - vor allem die Mehrwertsteuer und die Verbrauchsteuern auf Brennstoffe, Tabakwaren und alkoholische Getränke - wurden erhöht. Abgesehen von der Steuerreform von 1979 ist diese Verlagerung von direkten zu indirekten Steuern allmählich vonstatten gegangen. Ihre Auswirkungen seit 1979 lassen sich anhand der Abbildung 20 veranschaulichen.

${ }^{361}$ Giles und Johnson (1994), S. 79. 


\section{Abbildung 20: Die Zusammensetzung der britischen Steuereinnahmen 1979-1994}

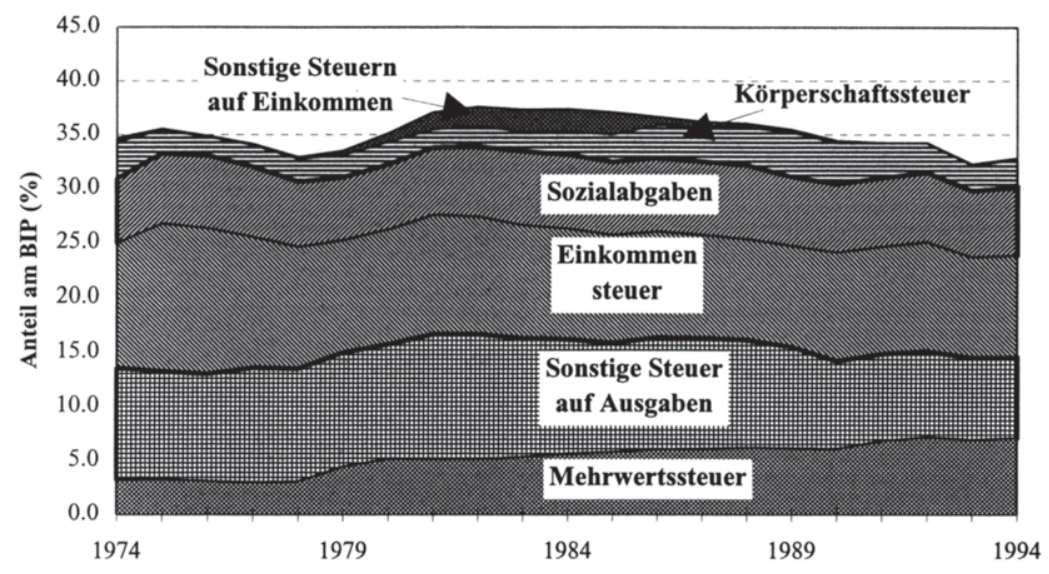

Quelle: CSO Blue Book, eigene Berechnungen

Der Anteil der Einkommensteuer ist um 1\% gesunken von 10,3\% 1979 auf 9,3\% 1994. Trotz der großen Tarifsenkungen haben sich die Einnahmen behauptet, was vor allem eine Folge der Ausdehnung der Bemessungsgrundlage war, aber auch für einen positiven Effekt von Steuersenkungen auf die Motivation und Leistung spricht. Dieser Effekt ist noch deutlicher bei der Körperschaftssteuer, deren Anteil trotz Tarifsenkungen von 2,1\% (1979) auf 2,6\% am BIP (1994) gestiegen ist. Die sonstigen direkten Steuern bestehen im wesentlichen aus Ölförderungsteuern und wurden bereits kommentiert. Die Sozialabgaben, insbesondere die Beiträge der Arbeitgeber und Niedriglohnverdiener, wurden in den 80er Jahren gesenkt. Erhöhungen der Arbeitnehmerbeiträge haben diese Entwicklung teilweise konterkariert. Das Verhältnis der Sozialabgaben am BIP ist bei circa 6,1\% relativ konstant geblieben. Bei den indirekten Steuern wird der großen Zunahme der Mehrwertsteuer in gestiegenen Einnahmen entsprochen. Die Quote lag 1979 bei 4,4\% und ist fast ununterbrochen auf 7,1\% im Jahre 1994 gestiegen. Dagegen haben Erhöhungen bei den sonstigen Verbrauchsteuern keine erhöhten Einnahmen bewirkt, das Aufkommen ist vielmehr um über $3 \%$ von $10,5 \%$ auf $7,4 \%$ gesunken. Diese Entwicklung kann teilweise auf sinkende Einnahmen aus dem Nordseeöl zurückgeführt werden, welches nicht nur mit direkten, sondern auch mit indirekten Steuern belastet wurde. 


\section{3) Der Beitrag der Staatsausgaben}

Angesichts der Bedeutung der Ausgabenziele für den Erfolg der MTFS (vgl. 3. Kapitel, Abschnitt III.2) wird der Beitrag der Staatsausgaben zur Konsolidierungsstrategie ausführlich untersucht. Nach einem Überblick des Verlaufs und der Ursachen der gesamten Ausgabenentwicklung werden die konkreten Maßnahmen zur Senkung der Ausgaben diskutiert. Anschließend wird die tatsächliche Entwicklung der Ausgaben analysiert aufgeteilt nach Aufgabenbereich, wirtschaftlichen Arten und Transferzahlungen.

\section{a) Die Entwicklung der gesamten Ausgaben}

Abbildung 21 zeigt die Entwicklung der Ausgabenquote (ohne Zinszahlungen auf die Staatsschuld) seit 1974. Im Vergleich zur Einnahmenquote (vgl. Abbildung 19) variierte im Betrachtungszeitraum die Ausgabenquote viel stärker. Insgesamt schwankte sie um über 6\%-Punkte. In ungefähr zehnjährigen Zyklen erreichten die Ausgaben 1974, 1984 und 1993 circa 41\% am BIP, dazwischen sanken sie stark. 1979 - dem Jahr der Konservativen Machtübernahme - lag die Ausgabenquote bei $37 \%$ und das Vorhaben der Konservativen Regierung, die Ausgaben nach ihrer Machtübernahme zu senken, wurde offensichtlich verfehlt. Erst nach der zweiten Wahlperiode und mit dem wirtschaftlichen Aufschwung konnte ab 1984 der Anstieg rückgängig gemacht werden. Bis 1989 sank die Ausgabenquote wieder und lag mit $34,8 \%$ circa $2 \%$ tiefer als zehn Jahre zuvor. Mit dem wirtschaftlichen Rückgang Ende der 80 er Jahre ist sie allerdings erneut stark und sehr schnell auf $41 \%$ angestiegen.

\section{Abbildung 21: Die Entwicklung der Staatsausgaben 1974-1994}

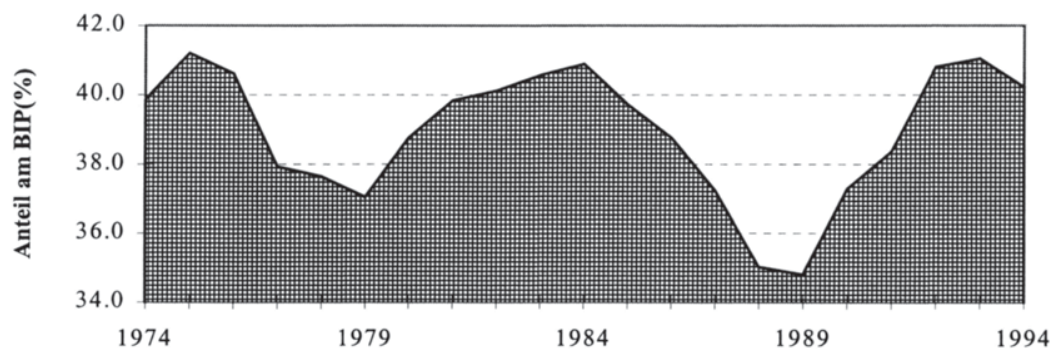

Quelle: CSO Blue Book, eigene Berechnungen 
Der Anstieg der Ausgaben hatte zwei wesentlichen Ursachen. Erstens war die Entwicklung offensichtlich stark vom Konjunkturzyklus geprägt. Die Ölpreiserhöhung von 1979 und die daraus resultierende Rezession, welche durch die restriktive Geld- und Fiskalpolitik vertieft wurde, ${ }^{362}$ erhöhten die zyklischen Ausgaben stark. Auch mit dem wirtschaftlichen Aufschwung ab circa 1982 ging der Anstieg der Ausgabenquote bis 1984 ununterbrochen, aber langsam weiter. Diese Entwicklung spiegelt diejenige der Beschäftigung wider. Die Arbeitslosigkeit stieg in der Rezession rasch von 5\% (1979) auf 9,8\% (1981) an. Danach nahm sie bis 1983 langsamer weiter zu, bis fast $12,4 \%$ der Erwerbspersonen arbeitslos waren. Bis 1987 blieb sie auf hohem Niveau. Schließlich sank sie dann bis 1990 auf 5,6\%, um in der Rezession 1993 erneut einen Höhepunkt bei 10,4\% zu erreichen. ${ }^{363}$ Die generell hohe und andauernde Arbeitslosigkeit dieser Periode verursachte nicht nur erhöhte Ausgaben für die Unterstützungszahlungen, sondern auch für Industrieförderung, Weiterbildungs- und Beschäftigungsprogramme. ${ }^{364}$

Zweitens gab es politische Gründe für den Anstieg. 1979 erforderten die abgegebenen Wahlversprechungen Ausgabenerhöhungen für Pensionszahlungen, Verteidigung, Gesundheitswesen, sowie Justiz und Polizei. ${ }^{365}$ Bei anderen Ausgabenkategorien, wie z. B. Bildung, waren Ausgabensenkungen in der Bevölkerung unbeliebt, weil ihnen Angebotseinschränkungen folgen mußten. ${ }^{366} 1982$ umfaßten die Wahlversprechungen Ausgabenerhöhungen für Verteidigung, soziale Sicherung, Beschäftigungsprogramme, Justiz und Polizei, Gesundheit, Bildung und Wissenschaft und kommunale Dienstleistungen. ${ }^{367}$ Nach der Wiederwahl der Konservativen Regierung 1983 waren die Anstrengungen zur Senkung der Ausgaben erfolgreicher. Die Ausgabenquote ging während des ganzen Aufschwungs bis $1989 \mathrm{zu}-$ rück - dies trotz wiederholter Versprechungen zu Ausgabenerhöhungen für Gesundheit, Beschäftigungsmaßnahmen, die innere Sicherheit und Pensionszahlungen während der Wahlkampagne 1987. Bei der folgenden Rezession und im Vorfeld der Wahl 1992 stieg die Ausgabenquote jedoch erneut sehr schnell an. Danach verlangsamte sich der Anstieg und nahm 1994 vom Höhepunkt 1993 bei 41\% wieder etwas ab.

\footnotetext{
362 Buiter und Miller (1983), S. $311 \mathrm{ff}$, Cairncross (1990) S. 241, Britton (1991) S. 54ff.

${ }^{363}$ Zur Entwicklung der Arbeitslosigkeit vgl. Abbildung 27.

${ }^{364}$ Ein Überblick dieser Programme findet sich bei Maynard (1988), Kapitel 6.

365 Vgl. Conservative Central Office (1977), S. 66.

${ }^{366}$ Caimcross (1992), S. 264.

${ }^{367}$ Conservative Central Office (1987), S. 19. 


\section{b) Maßnahmen zur Senkung der Ausgaben}

Wie im dritten Kapitel erläutert wurde, setzte die Strategie zur Senkung der Staatsquote einerseits auf makroökonomischer Ebene an mit den sogenannten „Cash Limits" für die geplanten Ausgaben (das Planning Total). Andererseits waren auf mikroökonomischer Ebene Maßnahmen zur Effizienzsteigerung im gesamten öffentlichen Sektor vorgesehen. Der oben erwähnte konjunkturelle und politische Druck auf die Ausgaben wirkte dann wie eine Nebenbedingung auf die Strategie und schränkte ihre Wirksamkeit erheblich ein. Zur Einhaltung der Ausgabengrenzen blieben für Kürzungen nur wenige Ausgabenbereiche übrig, in denen weder Ausgabenverpflichtungen noch Wahlversprechungen bestanden.

Damit mußte die Regierung große Hoffnung in eine erhöhte Effizienz der Staatstätigkeit setzen, die Einsparungen bei gleichbleibenden Leistungen ermöglichen sollte. Grundsätzlich werden Effizienzverbesserungen erreicht, wenn innerhalb des öffentlichen Sektors selbst sowie im Verhältnis zwischen öffentlichen und privaten Leistungsanbietern verstärkt der Wettbewerb als Mittel zur Kostenkontrolle eingesetzt wird. ${ }^{368}$ Die wichtigsten Instrumente dazu sind sogenannte Markttests und öffentliche Ausschreibungen - die öffentlichen Stellen müssen Angebote öffentlicher und privater Anbieter für ihre Dienstleistungen einholen und gegebenenfalls die Ausführung der Arbeiten dem privaten Sektor übertragen. Dies fand bei der Konservativen Regierung vor allem Anwendung bei den kommunalen Dienstleistungen sowie im Gesundsheitssektor. Im Durchschnitt wurden bei den öffentlichen Arbeiten, welche Markttests unterzogen wurden, Einsparungen von circa 17\% bis $25 \%$ erwirtschaftet. $^{369}$

Ebenfalls sehr bedeutsam für Effizienzsteigerung im öffentlichen Sektor sind Maßnahmen zur Arbeitsmarktreform im Sinne von Dienst- und Besoldungsreformen im öffentlichen Sektor. Dabei war die Senkung der öffentlichen Beschäftigung das zentrale Element in diesem Bereich, zumal die Regierungschefin Margaret Thatcher eine offene Antipathie für Bürokraten hegte und deswegen die Reduzierung des öffentlichen Dienstes zu einem eigenständigen Ziel machte. ${ }^{370}$ Allein die Privatisierung der öffentlichen Unternehmen führte zu bedeutenden Entlassungen aus dem öffentlichen Dienst, ${ }^{371}$ vor allem von Industriearbeitern. Öffentliche Ange-

${ }^{368}$ Hierzu OECD (1993b), S. $37 \mathrm{ff}$.

${ }^{369}$ Im Verhältnis zum Ausgabenvolumen wurden circa 7\% Markttests unterzogen, OECD 1989, S. $76 \mathrm{ff}$.

${ }^{370}$ Dunsire und Hood (1989), S. 15.

${ }^{371}$ Die Beschäftigung der öffentlichen Unternehmen sank von 2 Mio. 1979 auf 700.000 1991. Danach verdoppelte sie sich bis 1994 wieder (CSO, Blue Book). Letztere Entwicklung hing jedoch mit einer Umstrukturierung des öfentlichen Sektors zusammen. Sie wurde durch einen starken 
stellte - die Bürokraten im herkömmlichen Sinne - wurden weit weniger betroffen. ${ }^{372}$ Trotzdem ist die öffentliche Beschäftigung insgesamt von 7,5 Mio. (1979) auf 7,1 Mio. (1984) und dann auf 5,6 Mio. (1994), d. h. um 34\% zurückgegangen. ${ }^{373}$

Reformen der Lohn- und Gehaltsregelungen und der Beschäftigungsbedingungen wurden auch durchgeführt. Sie erstreckten sich von vorübergehenden Maßnahmen zur Dämpfung der Bezüge im öffentlichen Dienst, wie z. B. Ausgabengrenzen, über Besoldung nach Leistungsindikatoren und sonstigen Vergleichsmechanismen mit dem privaten Sektor, bis hin zu dauerhaften Reformen der Beziehungen zwischen den Tarifpartnern. ${ }^{374}$

Ferner ergriff die Regierung besondere Maßnahmen zur Einschränkung der Gemeindeausgaben, weil diese nach Ansicht der Regierung die geplanten Ausgaben nicht einhielten. ${ }^{375}$ Eine direkte Kontrolle über die Ausgaben der Gemeinde war zwar nicht möglich. Aber erstens wurden die Möglichkeiten der Gemeinden zur Mittelbeschaffung durch die verstärkte Kontrolle ihrer Schuld- und Steuerfinanzierung eingeschränkt. Zweitens sollte die Finanzierung der Kapitalausgaben möglichst durch Vermögensveräußerung abgedeckt werden. Insbesondere wurde 1980 das „Right to Buy“ Programm lanciert, welches die Mieter von Häusern im Staatsbesitz zum günstigen Kauf ermunterte.

Schließlich wurde eine Reform der sozialen Sicherung vorgenommen. Im Laufe der 80er Jahre wurden die Anspruchsrechte auf viele Leistungen - insbesondere die Unterstützung bei Erwerbslosigkeit - eingeschränkt und strengere Verfahren zur Überprüfung der Bedürftigkeit eingeführt. Zudem wurden Anfang der 80er Jahre implizite oder gesetzlich vorgeschriebene Anpassungen der Leistungen an die Lohnentwicklung oder sonstige Einkommensbegriffe abgeschafft und die Anpassungen der Leistungen eingestellt oder durch Preisindexierung ersetzt. Somit wurde das reale Niveau der Leistungen gesenkt oder zumindest eingefroren ${ }^{376}$ (vgl. hierzu auch 3. Kapitel, Abschnitt III.3.b).

Die Auswirkungen all dieser Maßnahmen auf die Entwicklung der Ausgaben sind in der aggregierten Ausgabenquote nicht zu erkennen, weil die konjunkturbeding-

Rückgang der zentralstaatlichen Beschäftigung von 900.000 im gleichen Zeitraum mehr als ausgeglichen.

372 Zwischen 1976 und 1986 betrafen lediglich 12,4\% des Personalabbaus die öffentlichen Angestellten, Dunsire und Hood (1989), S. 198ff.

${ }^{373}$ Circa 300.000 Beschäftigte in Arbeitsbeschaffungsmaßnahmen sind darin eingeschlossen.

${ }^{374}$ Hierzu OECD (1993c).

375 Conservative Central Office (1987), S. 19.

${ }^{376}$ Hierzu Webb (1995), S. 17 und Hills (1995b), S. 57füdith Safford - 978-3-631-75199-2 
ten Schwankungen davon isoliert und die Ausgaben weiter aufgeschlüsselt werden müßten. Deswegen werden für die weitere Analyse zwecks Vergleichbarkeit im Konjunkturverlauf ähnliche Perioden im Verlauf der Ausgabenentwicklung ausgewählt: in der Hochkonjunktur werden die Tiefpunkte der Ausgabenquote 1979 und 1989 miteinander verglichen, sowie die Jahre der höchsten Quoten 1984 und 1993. Zudem werden die Ausgaben in ihre verschiedenen Kategorien nach Aufgabenbereichen und anschließend nach wirtschaftlichen Arten getrennt. Zuletzt werden die Transferzahlungen behandelt.

\section{c) Die Verteilung der Ausgaben nach Aufgabenbereichen}

Abbildung 22 zeigt die Verteilung der Ausgaben nach Aufgabenbereichen in den vier Jahren 1979, 1984, 1989 und 1993. Ähnlich wie in der Bundesrepublik kommen den Ausgaben für soziale Sicherung das größte Gewicht zu. Die Ausgaben für Bildung und Gesundheit, einschließlich Sport und Erholung, nehmen ebenfalls einen großen Anteil ein. Letztes beruht auf der Verstaatlichung des Gesundheitswesens. Ebenso bedeutsam sind die Ausgaben für reine öffentliche Güter. Diese umfassen zu jeweils ungefähr der Hälfte die Ausgaben für die innere Sicherheit sowie für allgemeine Staatsleistungen, wie z. B. Parlament, Steuerbehörden, Außendienst und Verwaltung.

\section{Abbildung 22: Die britischen Staatsausgaben nach Aufgabenbereichen}

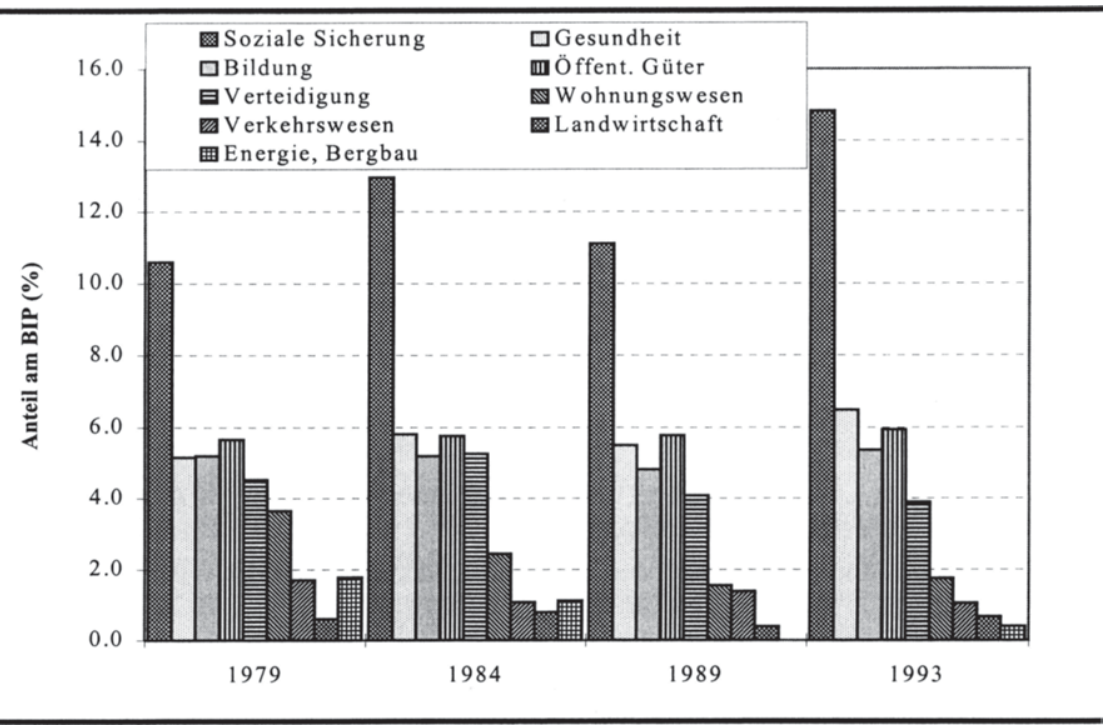


Abbildung 22 offenbart eine strukturelle Veränderung bei den Ausgaben. Erstens sind diejenigen für die soziale Sicherung als Anteil am BIP gewachsen: in den Vergleichsjahren der Hochkonjunktur 1979 und 1989 von $10,6 \%$ auf $11,1 \%$ und in den Vergleichsjahren der höchsten Ausgabenquote von $13 \%$ auf $14,8 \% .{ }^{377}$ Ein Teil dieser Entwicklung erklärt sich daraus, daß die Arbeitsmarktaufwendungen stark angestiegen sind. ${ }^{378}$ Im wesentlichen aber spiegelt der Anstieg die erhöhten Ansprüche an den Staat zur Erfüllung seiner Vor- und Fürsorgepflichten wider, die, wie im nächsten Kapitel gezeigt wird, eine Folge verschiedener Politikmaßnahme waren.

Auch die Ausgaben für den Gesundheitssektor sind gestiegen. Ihr Anteil am BIP erhöhte sich in den beiden Vergleichsjahren 1979 und 1989 von 5,2\% auf 5,5\%, und in denjenigen von 1984 und 1993 von 5,8\% auf $6,5 \%$. Die Bildungsausgaben blieben relativ konstant, obwohl die Anzahl der Schüler aus demographischen Gründen bis Ende der 80er Jahre um zwei Millionen gefallen ist. Gegen diesen Trend wirkte eine längere Schulausbildung (mehr Abiturienten) und ein steigender Anteil der Schulabgänger, die anschließend studierten. ${ }^{379}$ Schließlich haben die Ausgaben für öffentliche Güter im betrachteten Zeitraum ebenfalls geringfügig an Bedeutung gewonnen. Dies hängt vor allem mit einer bemerkenswerten öffentlichen Besorgnis über die sich ausweitende Kriminalität zusammen, ${ }^{380}$ welcher $u$. a. mit zunehmenden Ausgaben für die innere Sicherheit (Polizei, Gefängnisbau und Ähnliches) begegnet worden ist.

Die Ausgaben als Anteil am BIP sind gesunken in den Bereichen Wohnungswesen und kommunale Gemeinschaftsdienste, Verkehrs- und Nachrichtenwesen, sowie Energieversorgung und Bergbau, ${ }^{381}$ wobei die Einsparungen vor allem durch die Privatisierung dieser Aktivitäten ermöglicht wurde. Die Verteidigungsausgaben sind während des Falklands-Kriegs 1982 gestiegen, konnten aber danach gekürzt werden. Relativ unverändert blieben die Ausgaben für Landwirtschaft, Forstwirtschaft und Fischerei.

${ }^{377}$ Gemessen als Anteil an den gesamten Gesamtausgaben sind sie zwischen 1979 und 1993 sogar kontinuierlich angestiegen: von $27 \%$ auf $37 \%$.

${ }^{378}$ Im Vereinigten Königreich werden derartige Ausgaben direkt vom Zentralhaushalt getragen. Eine Bundesanstalt für Arbeit wie in der Bundesrepublik existiert nicht.

${ }^{379}$ Hibberd (1993a), S. 29.

380 Analysen der öffentlichen Meinung über Kriminalität und ihre Bekämpfung führten Dowds und Ahrendt (1996) und Brook und Cape (1996) durch.

${ }^{381}$ Siehe auch Cairncross (1992), S. 264f. 


\section{d) Die Verteilung der Ausgaben nach der Verwendungsrechnung}

Weitere Einsichten in die Entwicklung der Ausgaben lassen sich durch ihre Unterteilung nach wirtschaftlichen Charakter gewinnen. Zunächst kann zwischen den Staatsausgaben $\left(P_{t} G_{t}\right)$ im engeren Sinne, d. h. wo der Staat selbst im Markt tätig ist, und den Transferzahlungen $\left(\mathrm{Tr}_{\mathrm{t}}\right)$, wo der Staat lediglich Finanzmittel im privaten Sektor umverteilt, unterschieden werden. ${ }^{382}$ Die Staatsausgaben im engeren Sinne lassen sich weiter aufteilen in staatliche Konsum- und Investitionstätigkeiten sowie lokale und zentralstaatliche Ausgaben. Schließlich lassen sich die zentralstaatlichen Ausgaben in Lohnausgaben und Staatskäufe aufspalten. ${ }^{383}$ Abbildung 23 zeigt die Entwicklung der Staatsausgaben im engeren Sinne $\left(P_{t} G_{t}\right)$, unterteilt nach investiven und konsumtiven Bereichen. Dabei muß beachtet werden, daß die öffentlichen Investitionen sich unterschiedlich abgrenzen lassen. Die hier gewählte Definition umfaßt Ausgaben für Anlagevermögen, wie z. B. Grund und Boden, Gebäude und Maschinen. Dagegen werden Investitionen in Humankapital, wie z. B. für Bildung oder Forschung und Entwicklung, sowie in Verteidigungskäufe, welche zukünftige Leistungen verbessern, ${ }^{384}$ entsprechend den Bräuchen der britischen Statistik zu den laufenden Ausgaben gezählt. ${ }^{385}$ Die öffentlichen Investitionen werden auch als Netto-Investitionen betrachtet, $d$. h. der geschätzte laufende Verbrauch und Verschlei $\beta$ vom Vermögen werden abgezogen. Ebenfalls abgezogen werden Einnahmen aus dem Verkauf von Anlagevermögen. Deswegen ist es angesichts der Privatisierungspolitik und vor allem angesichts des Verkaufs der öffentlichen Wohnungsbauten sehr schwierig, ein Urteil über die Entwicklung der Kapitalausgaben zu fällen. Insofern sollte die Abbildung 23 mit Vorsicht interpretiert werden.

382 Transferzahlungen in Form von Zinszahlungen auf der öffentlichen Schuld werden getrennt weiter unten behandelt.

383 Die Trennung der Gemeindeausgaben in Lohnkosten und Staatskäufe läßt sich aus der Statistik des National Accounts (Blue Book) nicht ermitteln.

384 Hier unterscheidet sich die britische Vorgehensweise von der NATO-Abgrenzung, Hibberd (1993b), S. 64.

${ }^{385}$ Hierzu Hibberd (1993b), S. $56 f$. 


\section{Abbildung 23: Die Entwicklung der Ausgaben nach investiven und konsumtiven Bereichen}

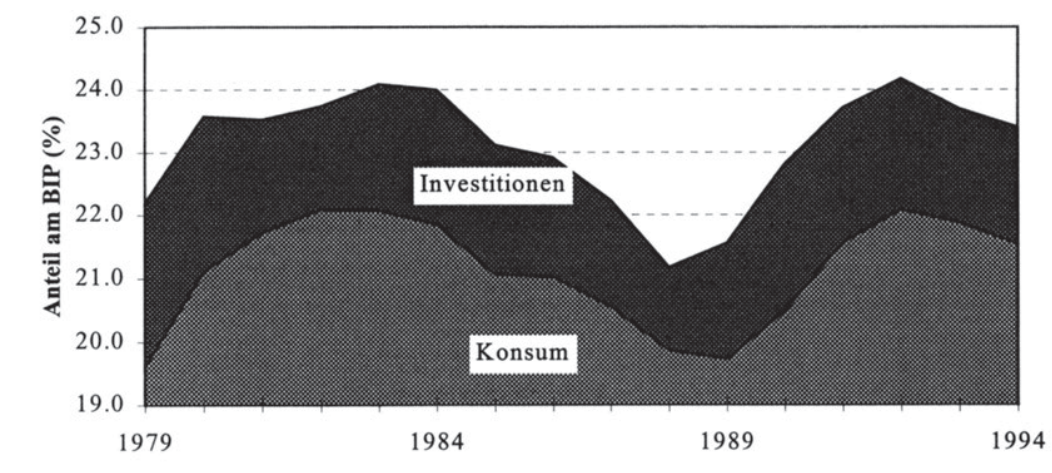

Quelle: CSO Blue Book, eigene Berechnungen

Trotz dieses Einwands läßt sich aus dem Diagramm schließen, daß die relativ starken Schwankungen der gesamten Quote vor allem auf die Entwicklung der Konsumausgaben zurückzuführen sind, die sehr stark variierten insbesondere mit einem markanten Rückgang zwischen 1983 und 1989. Die Investitionsquote veränderte sich weniger, von $2,6 \% 1979$ auf einen Tiefpunkt 1988 bei 1,3\%, und einen darauffolgenden leichten Wiederanstieg. ${ }^{386}$

Eine Aufgliederung der Staatsausgaben im engeren Sinne zeigt die Abbildung 24 für die ausgewählten vier Jahre. Dabei wird der zentralstaatliche Konsum in Lohnkosten und Staatskäufe aufgeteilt. Wegen der fehlenden Statistik ist dies für den Konsum der Gemeinden nicht möglich (vgl. Fußnote 383).

Die Unterteilung läßt erkennen, da $\beta$ der Rückgang der gesamten Investitionen vorwiegend auf sinkende Investitionen der Gemeinden zurückzuführen ist. ${ }^{387} \mathrm{Die}$ Gemeinden sind für die Investitionen in Nahverkehr und lokale Straßen, Wohnungswesen, lokale Dienstleistungen (z. B. für Freizeit und Erholung) und Bildung zuständig, ${ }^{388} \mathrm{~d}$. h. im wesentlichen für die Bereiche, in denen Einsparungen erreicht wurden.

386 Aus der Statistik des National Accounts (Blue Book) lassen sich die Bruttoinvestitionen nicht ermitteln. Nach einer Schätzung der OECD (1993b), S. 49, machte der Verkauf von Vermögen (Land, Wälder und Gebäude) jährlich bis zu 0,66\% am BIP aus, was bei einer Senkung der Nettoinvestitionsquote von 2,6 (1979) bis 1,3\% (1988) die Entwicklung etwas abmilderte.

${ }^{387}$ Zur Entwicklung der Kapitalausgaben im folgenden, siehe Hibberd (1993b).

${ }^{388}$ Stoker, G. (1988), S. 31. 
Allerdings brachte der Verkauf von sozialem Wohnungsbestand und sonstiges Vermögen den Gemeinden zwischen 1979 und 1994 Einnahmen von über $£ 42$ Mrd., ${ }^{389}$ welche in diesen Zeitreihen nicht erfaßt sind, aber circa $25 \%$ der Investitionen in diesem Zeitraum ausmachten. ${ }^{390}$

\section{Abbildung 24: Die Staatsausgaben nach der Verwendungsrechnung}

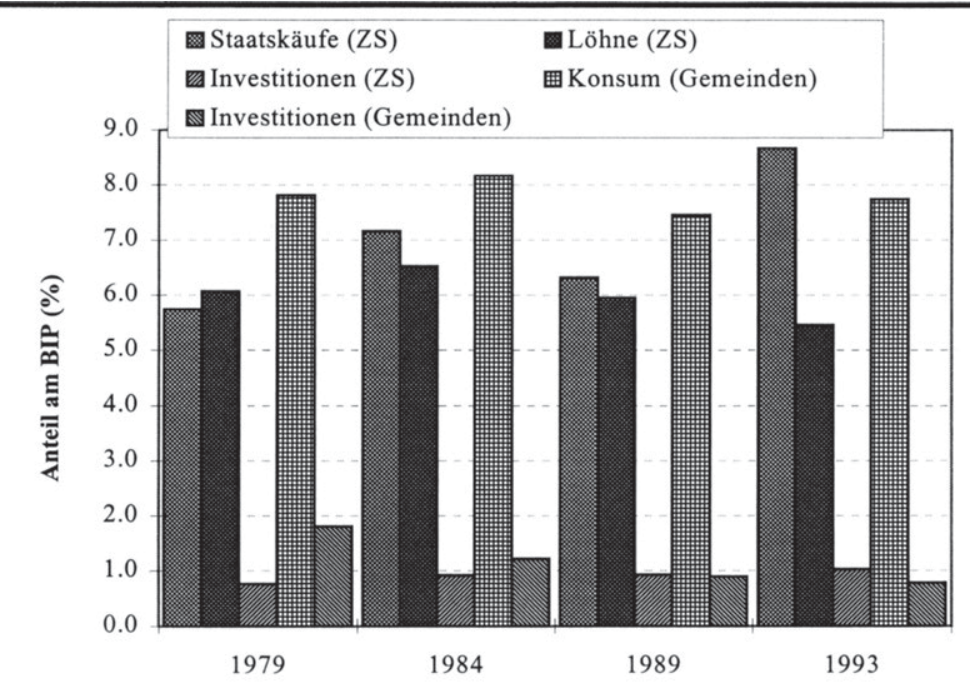

Merke: ZS bedeutet Zentralstaat; Quelle: CSO Blue Book, eigene Berechnungen

Die zentralstaatlichen Investitionen (ZS) sind relativ konstant geblieben. Diese Entwicklung ist jedoch ebenfalls teilweise der Veräußerung von Anlagevermögen $\mathrm{zu}$ verdanken. So haben beispielsweise in den 80er Jahren die Bruttokapitalausgaben für den Gesundheitssektor jährlich um real 3,75\% zugenommen. Der Bau von zusätzlichen Gefängnissen und Gerichtsgebäuden hat die Bruttokapitalausgaben für die innere Sicherheit um jährlich 13,2\% ansteigen lassen. Lediglich beim nationalen Straßenbau und bei den Verkehrsausgaben konnte die Wachstumsrate leicht unter derjenigen der Gesamtwirtschaft gehalten werden und somit eine geringe Senkung dieser Ausgaben erreicht werden. ${ }^{391}$

Der Konsum der Gemeinden als Anteil am BIP war höher in den beiden Rezessionsjahren 1984 und 1993 verglichen mit den beiden Hochkonjunkturjahren 1979

${ }^{389}$ CSO Blue Book, Tabelle 8.3.

${ }^{390}$ Wegen der fehlenden Jahres-Auflösung können sie in die Abbildung 24 nicht dargestellt werden.

${ }^{391}$ Hibberd (1993b), S. 62. 
und 1989. Zwischen den Vergleichsjahren (1979 und 1989 sowie 1984 und 1993) ist er jedoch geringfügig gesunken. Die erschwerten Finanzierungsmöglichkeiten sowie die Maßnahmen zur Effizienzsteigerung bei den kommunalen Dienstleistungen haben die Ausgabenentwicklung offenbar gedämpft. Einsparungen konnten vor allem beim Nahverkehr und dem Straßenbau erzielt werden.

Der zentralstaatliche Konsum wird aufgeteilt in Lohnkosten und Staatskäufe. Erstere stiegen von 6,1\% (1979) und auf 6,5\% (1984). Diese ansteigenden Lohnkosten trotz des Abbaus von 0,4 Mio. Stellen (vgl. S. 142) lassen sich politisch erklären. Während ihrer Wahlkampagne im Jahre 1978 hatte die Regierung versprochen, die Empfehlungen der Königlichen Untersuchungskommission (der sogenannten Clegg Commission) über Tarife im öffentlichen Sektor zu respektieren. Nachdem in den 70er Jahren die Lohn- und Einkommenspolitik bei hohen Inflationsraten zu erheblichen Reallohnsenkungen geführt hatte, empfahl die Kommission Tariferhöhungen von durchschnittlich $25 \%$ im Finanzjahr 1979/80, was demzufolge erhebliche Mehrausgaben verursachte. ${ }^{392}$ Nach 1984 gingen die Lohnkosten zurück bis auf 4,4\% des BIP im Jahre 1994. Mit anderen Worten, der Abbau der öffentlichen Beschäftigten um fast 2 Mio. oder um 34\% im Untersuchungszeitraum ermöglichte lediglich eine Senkung der Lohnkosten um 1,7\% am BIP. Ohne in diesem Rahmen

auf eine tiefere Analyse dieser Entwicklung eingehen zu können, ${ }^{393}$ läßt sich für die Zwecke dieser Arbeit feststellen, daß der enorme Abbau des öffentlichen Dienstes insgesamt wenig zur erhofften Ausgabensenkung beizutragen vermochte.

Darüber hinaus wurde diese geringe Senkung der zentralstaatlichen Lohnkosten durch einen starken Anstieg der Staatskäufe mehr als kompensiert. Gemessen am BIP stiegen sie in den Vergleichsjahren von 5,7\% (1979) auf 6,3\% (1989) und von 7,2\% (1984) auf 8,7\% (1993). Die Ursachen hierfür wurden bei der Analyse der Ausgabenkategorien (Abbildung 22) ersichtlich: Sie liegen beim Anstieg der Ausgaben für die innere Sicherheit, für das zentralstaatliche Bildungsangebot (im wesentlichen eine Beteiligung an den Einrichtungen der höheren Bildungsstätten, d. h. Universitäten und Fachhochschulen), und für das Gesundheitswesen. Weiter läßt sich daraus schließen, daß die eingeleiteten Effizienzmaßnahmen den Ausgabenanstieg nicht verhindern konnten.

\section{e) Die Entwicklung der Transferzahlungen}

Gewisse Ähnlichkeiten zu den Staatsausgaben $\left(P_{t} G_{t}\right)$ lassen sich bei der Entwicklung der Transferzahlungen feststellen. Diese sind in Abbildung 25 nach den lau-

392 Buiter und Miller (1981), S. 327.

393 Siehe hierzu Dunsire und Hood (1989), Kapitel 8 sgwieftFry (1988)- 978-3-631-75199-2 
fenden und den sogenannten investiven Übertragungen (d. h. Subventionen) aufgeteilt. Insgesamt sind die Transferzahlungen im Verhältnis zum BIP geringfügig angestiegen. Die Subventionen, welche der Industrie und Landwirtschaft zuflossen, blieben im ersten Jahrzehnt insgesamt relativ konstant (1979 0,9\%; 1989 0,8\%). Im Jahre 1990 verdoppelten sie sich auf 1,8\% am BIP und blieben dann auf etwas höherem Niveau. Diese Entwicklung war zu Beginn die Folge der Unterstützungszahlungen an die beiden Unternehmen Rover und Short Brothers sowie der Subventionierung des Schuldendienstes der Elektrizitätsunternehmen vor und während ihrer Privatisierung. Das höhere Niveau wurde danach durch Subventionen an die staatlichen Transportunternehmen British Rail und London Transport gehalten. ${ }^{394}$

Die laufenden Transfers schwankten ähnlich wie der Staatskonsum, sehr stark im Konjukturzyklus. Vor allem nahmen sie ab 1980 rapide zu. Zwischen 1982 und 1985 verharrten sie auf hohem Niveau, um dann bis 1989 rasch wieder abzusinken. Danach nahmen sie erneut stark zu. 1993 erreichten sie ihren höchsten Stand mit $16 \%$. Um diese Schwankungen zu interpretieren, ${ }^{395}$ ist zunächst zu berücksichtigen, daß viele Transferzahlungen stark von der Entwicklung der Beschäftigung abhängig sind, da die Anzahl der Transferempfänger in Zeiten hoher Arbeitslosigkeit steigt. Die Entwicklung der Arbeitslosenrate im Vereinigten Königreich zwischen 1979 und 1994 wurde durch die Entwicklung der persönlichen Transferzahlungen widergespiegelt, wie die Abbildung 25 auch zeigt.

\section{Abbildung 25: Die Entwicklung der laufenden Transferzahlungen und der Subventionen}

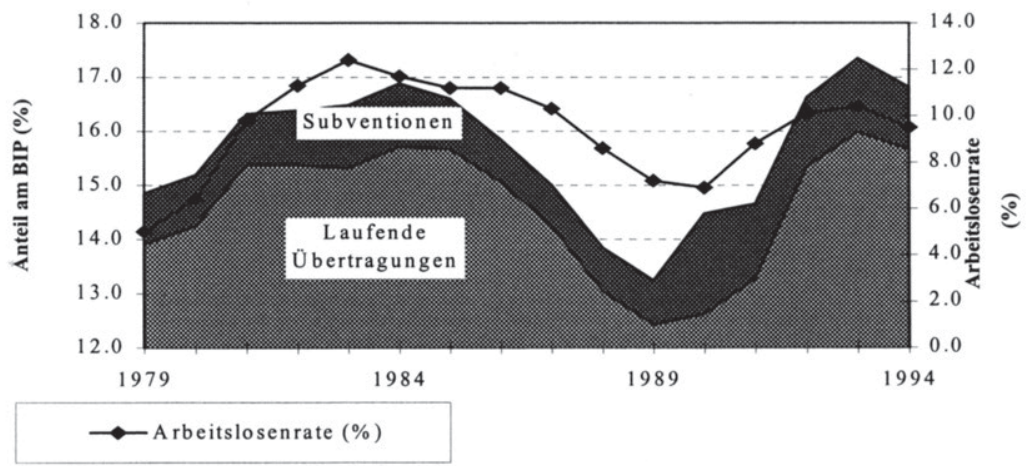

Quelle: CSO Blue Book, OECD 1996, Tab. 22 und eigene Berechnungen

${ }^{394}$ Hibberd (1993b), S. 60.

${ }^{395}$ Hierzu Hibberd (1993a), S $28 \mathrm{ff}$. 
Die laufenden Übertragungen bestehen im wesentlichen aus personellen Transferzahlungen an die Haushalte und ihr Anstieg ist auch allein auf diese zurückzuführen. ${ }^{396}$ Um ihre Entwicklung zu untersuchen, werden sie in Abbildung 26 nach Transferarten aufgeteilt.

Aus der Abbildung 26 wird neben der Beschäftigungsentwicklung ein zweiter Faktor für die Entwicklung der Transferzahlungen erkennbar. Wie in den meisten OECD-Ländern beanspruchen die Pensionszahlungen einen hohen Anteil der Transfers. Dies ist demographisch bedingt durch die zunehmende Zahl der Rentenempfänger sowie deren zunehmender Lebenserwartung. Zwischen 1979 und 1994 nahm die Anzahl von Rentnern um 5,5\% zu und die Anzahl von Rentenempfängern über 75 Jahre um 24\%. Ältere Menschen erhielten auch einen relativ hohen Anteil der gestiegenen Zahlungen für Pflegeaufwand und Behindertenrenten.

\section{Abbildung 26: Die Entwicklung der persönlichen Transferzahlungen}

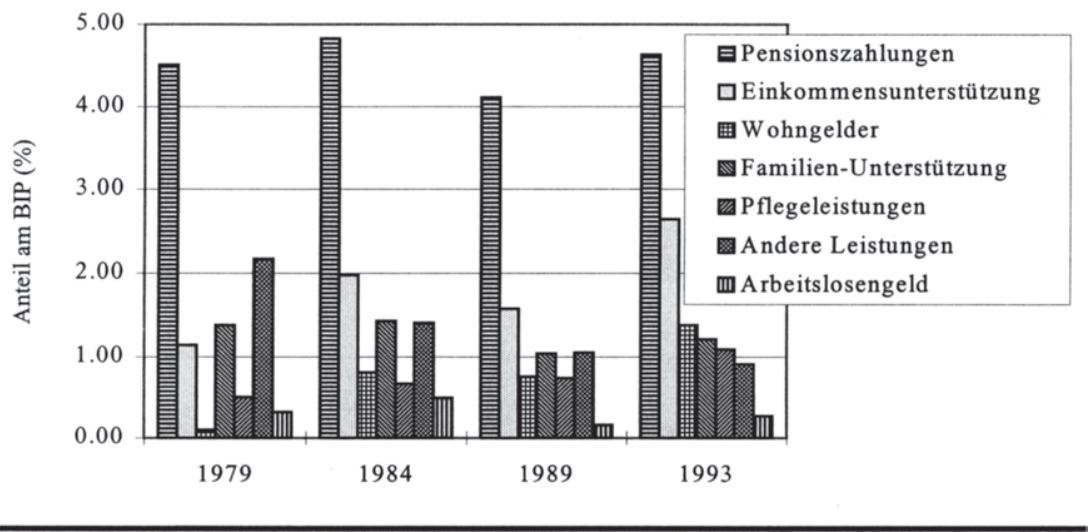

Quelle: CSO Blue Book, eigene Rechnungen

Dennoch sind, überraschenderweise, die Rentenzahlungen in den jeweiligen Vergleichsjahren (1979 und 1989, sowie 1984 und 1993) als \%-Anteil am BIP zurückgegangen. Gesenkt wurde auch der Anteil für Familienunterstützung, für das Arbeitslosengeld und für die sogenannten „Anderen Leistungen.“ Diese umfassen etwa zur einen Hälfte verschiedene Sonderleistungen im Todes-, Invaliditäts- oder Krankheitsfall, für Witwenrenten, Mutterschaftsgelder und Ähnliches, sowie zur

${ }^{396}$ Die Transferzahlungen an das Ausland machen durchschnittlich 0,9\% am BIP aus und sind geringfügig gesunken. Die laufenden Subventionen machten 1979 2,3\% am BIP aus und sanken stetig auf $1,1 \%(1994)$. 
anderen Hälfte das allgemeine Kindergeld. In diesen Bereichen hat die Konservative Politik einem Anstieg der Transferzahlungen als Anteil am BIP Einhalt gebieten können. ${ }^{397}$

Gestiegen ist dagegen der Anteil in den drei Kategorien Pflegeleistungen, Wohngelder und Einkommensunterstützung. Pflegeleistungen werden vor allem an ältere Menschen im Pflegefall bezahlt, und ihr Anstieg ist, wie bereits erwähnt, demographisch zu erklären. Wohngelder und Einkommensunterstützung sind beitragsunabhängige Zahlungen. Sie dienen zur Sicherung der Mindestbedürfnisse von Leistungsabhängigen, die keinen Anspruch auf sonstige Unterstützungszahlungen erheben können. Die Einkommensunterstützung entspricht der deutschen Sozialhilfe. Die häufigsten Empfänger sind Rentner, die keine anderen Einkünfte als die staatliche Rente beziehen. Alleinerziehende und ihre Kinder sind ebenfalls häufig auf beitragsunabhängige Unterstützungszahlungen angewiesen. Anfang der 90er Jahre waren zwei Drittel alleinerziehender Eltern von staatlicher Unterstützung abhängig, verglichen mit einem Drittel zehn Jahre zuvor. Schließlich erhalten viele Arbeitslose auch Wohngelder und Einkommensunterstützung, wenn sie keinen Anspruch auf Arbeitslosengeld geltend machen können. Arbeitslosengeld wird für höchsten ein Jahr gewährt, wenn Beiträge während der vorherigen zwei Jahre geleistet wurden. Jugendliche, Selbständige und Frauen haben häufig ungenügende Beiträge in die Arbeitslosenversicherung geleistet. Langzeitarbeitslose und ältere Erwerbslose (über 60) erhalten auch keine Arbeitslosengelder mehr. Dies hat zur Folge, daß insgesamt $75 \%$ der Arbeitslosen vom Arbeitslosengeld ausgeschlossen sind. ${ }^{398}$

Diese Verschiebungen bei den Transferzahlungen sind zum einen das Ergebnis der oben erwähnten Reform des Wohlfahrtsstaats. ${ }^{399}$ Senkungen waren in einigen Bereichen durchaus möglich. Gleichzeitig haben die Reformen zu Verschiebungen innerhalb der persönlichen Transferzahlungen geführt, weil viele Empfänger von den beitragsabhängigen Leistungen ausgeschlossen wurden und deswegen die Leistungen des Sozialnetzes in Form von beitragsunabhängigen Unterstützungsgeldern in Anspruch nehmen mußten. ${ }^{400}$

Zum zweiten hat eine Zunahme der Armut im Vereinigten Königreich im Laufe der 80er Jahre zu einer erhöhten Beanspruchung dieser Transferzahlungen geführt. Der Bevölkerungsanteil mit einem Einkommen unter der Hälfte des durchschnittlichen Einkommens stieg von 4,5 Mio. oder circa 10\% (1979) auf 11,4 Mio. oder 20\%

\footnotetext{
${ }^{397}$ Insgesamt machten diese Transferzahlungen $19798,3 \%$ und $19897,1 \%$, sowie $19847,2 \%$ und $19936,2 \%$ am BIP aus.

398 Siehe hierzu Haskel und Jackman (1988), S. 380f., sowie Ashby (1985), S. 1f.

${ }^{399}$ Siehe hierzu auch den Sammelband Dilnot und Walker (Hrsg.) (1989).

${ }^{400}$ Hierzu insbesondere Bradshaw und Huby (1989). 
(1991) an. ${ }^{401}$ Diese Bevölkerungsgruppe ist in der Regel von staatlichen Unterstützungszahlungen abhängig. 1979 erhielten beispielsweise vier Millionen Menschen Einkommensunterstützung, 1994 waren es zehn Millionen. ${ }^{402}$

\section{4) Die Zinszahlungen für die Staatsschuld}

Die letzte Komponente der Budgetbeschränkung umfaßt die Zinszahlungen auf die Staatsschuld. Obwohl diese keine Politikvariablen sind, werden sie in diesem Abschnitt behandelt, da sie den Budgetspielraum entscheidend beeinflussen. Die nominale Zinslast als Anteil am BIP ist in der Abbildung 27 veranschaulicht; sie ist nach einem kurzen Anstieg von 1979 bis 1982 stark gefallen. 1982 machte sie 5\% am BIP aus. Zehn Jahre später sank sie auf ihren tiefsten Punkt von 2,9\%. Nur in diesem und im folgenden Jahr (1993) fiel sie unter die 3\% Grenze des Maastrichter Vertrags für das sekundäre Defizit und ließ somit noch einen minimalen Raum für Neuverschuldung übrig. Mit anderen Worten: Selbst nach den enormen Konsolidierungsbemühungen der 80er Jahre wurde praktisch der gesamte Spielraum des Maastrichter Defizitkriteriums durch den Schuldendienst auf die vergangene Kreditaufnahme beansprucht.

Ebenfalls in der Abbildung eingetragen ist die Zinsentwicklung für Staatspapiere mit einer zehnjährigen Laufzeit, (was ungefähr der durchschnittlichen Restlaufzeit der britischen Staatsschuld entspricht). Die rückläufige Zinsentwicklung seit 1981 konnte zur Senkung der Zinskosten beitragen.

\footnotetext{
401 Goodmann und Webb (1994), S. 39ff. Nach einer üblichen Messung werden alle Haushalte mit einem Einkommen unter der Hälfte des Durchschnittseinkommens (Mittelwert) als arm bezeichnet, Atkinson (1983), S. 238f.

402 Webb (1995), S. 16. 


\section{Abbildung 27: Die Brutto-Zinszahlung auf die Staatsverschuldung}

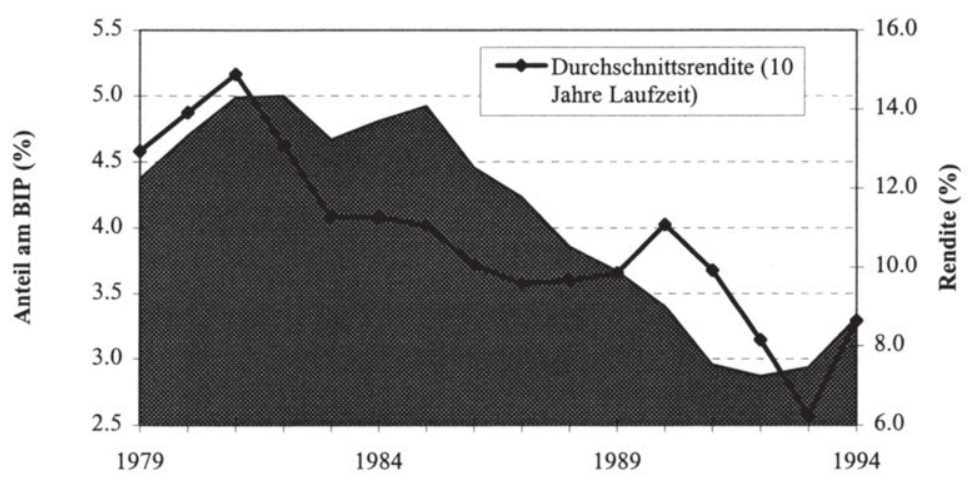

Quelle: CSO Blue Book, OECD Country Survey und eigene Berechnungen

Im dritten Kapitel (Abschnitt IV.3.a) wurde gezeigt, wie die mittelfristige Finanzstrategie der Konservativen Regierung eine Verbindung zwischen Budgetdefizit und Inflationsrate herstellte, bei der die geplante staatliche Kreditaufnahme durch den Tilgungseffekt der Inflation real vernichtet wurde $\left(B_{t}-B_{t-1}=\pi_{t} B_{t-1}\right)$.

Die Zinszahlungen auf die Staatsschuld setzen sich aus einer realen Komponente und einem Inflationsausgleich zusammen: $i_{t-1} B_{t-1}=r B_{t-1}+\pi_{t} B_{t-1}$. Gemäß der mittelfristigen Finanzstrategie kann der Inflationsausgleich durch die Neuverschuldung finanziert werden. $\mathrm{Zu}$ finanzieren bleibt damit noch die reale Zinslast $\left(\mathrm{rB}_{\mathrm{t}-1}\right)$.

Folglich muß für eine Stabilisierung der Schuldenquote der Primärüberschuß nicht die ganzen nominalen Zinszahlungen decken, sondern lediglich die reale Zinslast $\left(P_{t} G_{t}+T_{t}-T_{t}=r B_{t-1}\right)$. Durch den Abzug der im Abschnitt I.3 berechneten Inflationstilgung von den obigen nominalen Zinszahlungen wird eine Vorstellung dieser realen Zinslast erhalten $\left(\mathrm{iB}_{\mathrm{t}-1}-\pi \mathrm{B}_{\mathrm{t}-1}=\mathrm{rB}_{\mathrm{t}-1}\right)$. Diese wird dann in Abbildung $28 \mathrm{mit}$ dem Primärsaldo verglichen, um dessen Deckungsgrad gegenüber der realen Zinslast abzuschätzen. 


\section{Abbildung 28: Der Deckungsgrad der Realzinslast durch den Primäruberschuß 1979 bis 1994}

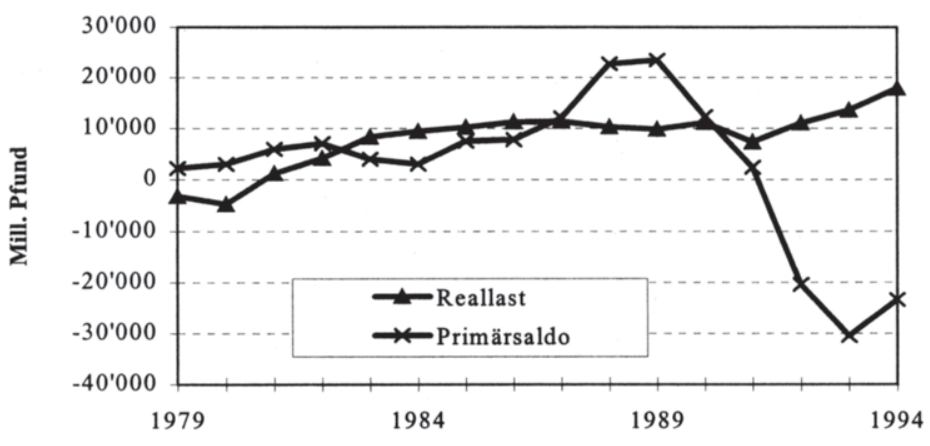

Quelle: CSO Blue Book, eigene Berechnungen

Zwischen 1979 und 1982 sowie erneut von 1987 bis 1990 reichte der Primärüberschuß aus, um die so berechnete Reallast der Verschuldung abzudecken. Zwischen 1983 und 1986 fiel die Inflationstilgung niedriger aus, und das Primärsaldo deckte die reale Last nicht ab. Zwischen 1991 und 1994 schlußendlich geriet bei weiter steigender Realzinslast das Primärsaldo in ein starkes Defizit - von Deckung keine Rede mehr.

\section{Zusammenfassung des vierten Kapitels}

In diesem Kapitel wird die Entwicklung der Staatsfinanzen untersucht und insbesondere die jeweiligen Beiträge der Bestandteile der Budgetbeschränkung zum Abbau der Staatsverschuldung ermittelt. Im ersten Abschnitt werden die Auswirkungen des realen Wachstums und der Inflation auf die Entwicklung der Schuldenquote geschätzt. Unter Berücksichtigung der Jahre des negativen Wachstums hat das Wachstum insgesamt circa 14 Prozentpunkte oder jährlich im Durchschnitt 1\% zur Senkung der Schuldenquote beigetragen. Die Auswirkungen der Inflation fielen wesentlich stärker aus. Vor allem am Anfang der Periode (1979-1981), als die Inflation zweistellig war, bewirkte diese eine effektive Tilgung der Staatsverschuldung. Im Periodendurchschnitt reichte diese Tilgung aus, um den Wert der Neuverschuldung auszugleichen. 
Im zweiten Abschnitt werden die jeweiligen Beiträge der Finanzierungskomponenten der Budgetbeschränkung ermittelt. Die Einnahmen aus der Geldschöpfung fallen sehr gering aus. In Übereinstimmung mit ihrer Strategie der Inflationsbekämpfung hat die Konservative Regierung nicht gezielt auf diese inflationäre Finanzierungsquelle zugegriffen. Dagegen erzielte die Konservative Regierung einen wesentlichen Finanzierungsbeitrag durch die Veräußerung des staatlichen Vermögens bei ihrer Privatisierungspolitik. Dadurch konnte die öffentliche Neuverschuldung nach 1983 jährlich um ungefähr 1\% des BIP reduziert werden. Die tatsächliche Neuverschuldung durch die Ausgabe von staatlichen Schuldpapieren, Schatzpapieren und sonstigen Sparbriefen machte im Durchschnitt 2,3\% am BIP aus.

Im dritten Abschnitt wird die Entwicklung der Primär- und Sekundärsaldi untersucht. Der Primärsaldo war von 1979 bis 1991 im Überschuß. Damit haben die politischen Maßnahmen in dieser Zeit nicht zur Neuverschuldung beigetragen. Ab 1992 geriet der Primärsaldo jedoch stark ins Defizit. Die detaillierte Untersuchung der Einnahmekomponenten offenbart den wichtigen Beitrag der Einnahmen aus dem Nordseeöl in den achtziger Jahren. Trotz umfassender Reformen, einschließlich einer beträchtlichen Senkung der Einkommensteuersätze, bleiben die anderen Steuereinnahmen relativ konstant.

Die Entwicklung der Ausgaben wird durch starke Schwankungen gekennzeichnet. Sie scheint im besonderen von der Beschäftigungsentwicklung beeinflußt zu sein. Die Ausgaben für die soziale Sicherung, Gesundheit und die Leistungen für Verwaltung, die innere Sicherheit sind jedoch auch stetig gestiegen und entwickelten sich unabhängig von der Arbeitslosenzahl. Trotz eines absoluten Rückgangs der Schülerzahlen waren auch die Ausgaben für Bildung nicht rückläufig.

Der Großteil der Ausgaben für die soziale Sicherung werden für Transferzahlungen aufgewendet. Obschon Reformmaßnahmen ergriffen wurden in bezug auf die Berechtigung auf staatliche Aufwendungen sowie auf ihre Höhe, stiegen die persönlichen Transferzahlungen als Anteil am BIP seit 1979. Eine Zunahme der Leistungsberechtigten überkompensierte die Kürzungen der Leistungen.

In einigen Bereichen konnte die Regierung Einsparungen durchsetzen und damit den Aufwärtstendenzen Ausgabenkürzungen entgegensetzen. Die Ausgaben für Verkehr, Wohnungswesen und Energie sanken als Anteil am BIP. Hinter dieser Entwicklung stand die Privatisierung vieler Träger in diesen Bereichen sowie eine Senkung der investiven Ausgaben, insbesondere der Gemeinden. Letztere wurden durch die Veräußerung des sozialen Wohnbaubestandes ermöglicht.

Schließlich konnte die Last der Zinszahlungen auf die Staatsverschuldung im Verhältnis zum BIP um über 3\% gesenkt werden. Gegen Ende der Periode stieg infolge der erneuten Kreditaufnahme ab 1991 die Zinslast wieder an. 
Judith Safford - 978-3-631-75199-2

Downloaded from PubFactory at 01/11/2019 07:12:45AM

via free access 


\section{Fünftes Kapitel}

\section{KONSEQUENZEN UND PERSPEKTIVEN DER KONSOLIDIERUNGSPOLITIK IM VEREINIGTEN KÖNIGREICH}

Im folgenden sollen die Ergebnisse des vorhergehenden Kapitels mit den im zweiten Kapitel entwickelten Kriterien für die Abbaustrategien verglichen werden. Dies soll ein Urteil über den Erfolg der Strategie des Schuldenabbaus und die Perspektiven der zukünftigen Entwicklung ermöglichen. Zuerst werden die jeweiligen Beiträge der staatlichen Einnahmen zum Schuldenabbau überprüft und ihre zukünftige Bedeutung für die Staatsfinanzen eingeschätzt. Im zweiten Abschnitt wird der Anstieg der Ausgaben wieder aufgegriffen. Die Finanzierungsfrage wird im dritten Abschnitt erneut unter Berücksichtigung der Ausgabenentwicklung untersucht.

\section{Die Entwicklung der Staatseinnahmen}

\section{1) Der Wegfall der Inflation als Lösung des Schuldenproblems}

Bei ihrem Amtsantritt hatte sich die Konservative Regierung zur Beseitigung der Inflation verpflichtet. Auch wenn ihr dieses Ziel nicht vollkommen gelungen ist, so sank die Inflation im Laufe der 80er Jahre doch stark. Die Ziele der MTFS, die niedrige Seignorage, die Vorkehrungen gegen Inflationsgewinne durch das Steuersystem, sowie der hohe Anteil von indexierten Staatsschuldpapieren machten die Haltung der Konservativen Regierung gegen die Inflation glaubwürdig. Sie hat die Inflation auch nicht gezielt zur Reduktion der Staatsverschuldung eingesetzt. Das bedeutet aber nicht, daß die Inflation sich nicht vorteilhaft auf die Schuldenquote ausgewirkt hätte. Im Gegenteil: Zwischen 1979 und 1994 hat die Inflation die Schuldenlast enorm gemindert. Die fallenden Inflationsraten vor allem gegen Ende der Periode bedeuteten jedoch, da $\beta$ diese Finanzierungsquelle immer unwichtiger wurde. Wenn die Regierung in ihrer Inflationsbekämpfung erfolgreich bleibt, wird der Druck zu Einsparungen auf die anderen Einnahmenquellen, bzw. auf die Ausgaben in der Zukunft zunehmend größer. ${ }^{403}$

${ }^{403}$ Reales Wachstum hat in den 80 er Jahren zur Senkung der Schuldenquote beigetragen (vgl. drittes Kapitel, Abschnitt I.2) und kann auch einen wichtigen Beitrag zur zukünftigen Konsolidierung 


\section{2) Erlöse aus der Privatisierung}

Die Privatisierungserlöse konnten in den 80er Jahren die Staatsfinanzen ebenfalls ganz wesentlich entlasten. Dieser Finanzierungsquelle sind naturgemä $B$ durch das Ausmaß des veräußerbaren öffentlichen Vermögens Grenzen gesetzt, und im wesentlichen ist sie jetzt erschöpft. Noch beabsichtigt die Regierung, die staatliche Eisenbahn und die Kernenergieanlagen zu veräußern. Beide Unternehmen sind nicht rentabel und bedürfen erheblicher Investitionen und/oder Subventionen vor dem Verkauf. Seit 1989 wurden die Investitionen für die Eisenbahn von anfänglich circa $£ 0,5$ Mrd. bis 1992 real verdreifacht. ${ }^{404}$ Dem stehen bisher (Ende 1996) Erlöse von $£ 1,1 \mathrm{Mrd}$. für das Bahnnetz gegenüber. ${ }^{405}$ Der Verkauf der Kernenergieanlagen scheiterte schon 1990, weil die Folgekosten bei der Endlagerung der Abfallprodukte und andere Produktionsrisiken diese Energieträger aus Sicht des privaten Investors unrentabel machten. Mit Preisabschlägen und sonstigen Subventionen ging das Unternehmen der Kernenergie seit Sommer 1996 an die Londoner Börse. ${ }^{406}$ Die hohen Investitionen und Subventionen dieser Unternehmen lassen substantielle Nettoeinnahmen infolge ihrer Privatisierung fragwürdig erscheinen.

In den 80er Jahren erzielten die Gemeinden beträchtliche Einnahmen aus der Veräußerung von sozialem Wohnungsbestand. Der Restbestand hat einen geschätzten Verkehrswert (1993) von $£ 40$ Mrd. ${ }^{407}$ Mit der Erholung des Wohnungsmarktes nach der Rezession von Anfang der 90er Jahre können die Gemeinden diese Verkäufe fortsetzen. Allerdings wurde die Nachfrage nach Wohneigentum durch die günstigen Verkaufsbedingungen der 80er Jahre im wesentlichen bereits befriedigt. $^{408}$

Eine bisher kaum erschlossene Einnahmequelle ist der Verkauf von staatlichen Grundstücken und Gebäuden. ${ }^{409}$ Eine Budgetentlastung erbrächte beispielsweise der Verkauf der Londoner Grundstücke und Gebäude aus dem Besitz der Gesundheits- und Verteidigungsministerien, sowie des Kronbesitzes, welcher neben Büro-, Wohn- und Industriegebäude auch Agrarland und Küste mit einem Verkehrswert von schätzungsweise £2,3 Mrd. umfaßt. Nach Ansicht von einigen Regierungskreisen könnten die daraus gewonnenen Mittel weitere Steuersenkungen finanzieren.

\footnotetext{
leisten. Ein politisches Instrument hierzu ist es jedoch nicht und wird deswegen nicht weiter betrachtet.

${ }^{404}$ Hibberd (1993b), S. 70.

${ }^{405}$ Financial Times, 16.6.96, S. 1.

${ }^{406}$ Financial Times, 5.6.96, S. 1 sowie 27.6.96, S.1.

407 Wilcox (1994), S. 45.

408 Wilcox (1994), S. 44.

${ }^{409}$ Hierzu Economist, 31.10.96, S. 31 f. 
Gerade unter dem Druck von derartig kurzfristigen politischen Überlegungen besteht jedoch die Gefahr, daß das Vermögen unterbewertet verkauft wird. Wie die Analyse im zweiten Kapitel zeigt, stellt der Verkauf vom unterbewertetem Vermögen langfristig eine Budgetbelastung dar, weil die kurzfristigen Erlöse die langfristigen Vermögenserträge nicht kompensieren. Nach verbreiteter Ansicht wurde das britische Staatsvermögen häufig zu unterbewerteten Preisen veräußert. ${ }^{410}$ Dies ist wohl mit ein Grund für die Verschlechterung der staatlichen Nettovermögensposition seit Anfang der 80er Jahre. ${ }^{411}$

Auch wenn starke Argumente für die Privatisierung des öffentlichen Vermögens existieren, ist es nicht erwiesen, daß sie langfristig gesehen ein sinnvolles Instrument zur Budgetfinanzierung ist. Privatisierungseinnahmen befreien den Staat auch nicht von dauerhaften Budgetreformen. Die Aufnahme der Privatisierungserlöse in die Ausgabenvorgaben des „Planing Total“ hat aber gerade dazu verleitet, die Ausgabenziele durch Privatisierungseinnahmen einzuhalten und dauerhafte Budgetreformen zu umgehen. In dieser Hinsicht ist die neue Plangröße für die Ausgaben seit 1993 - das „New Control Total“ eine positive Entwicklung, weil es die Privatisierungserlöse nicht enthält.

\section{3) Steigende Steuereinnahmen}

Die Steuererträge wurden in den 80er Jahren weiter auch durch einmalige Einnahmen erhöht. Verschiedene Steuern auf das geförderte Nordseeöl brachten hohe Einnahmen und damit eine große Entlastung der öffentlichen Finanzen. Mit der Erschöpfung der Ölressourcen fielen diese Einnahmen seit Anfang der 90er Jahre weitgehend weg.

Die sonstigen Steuereinnahmen blieben insgesamt relativ konstant, und es wurde nicht versucht, durch erhöhte Steuern zum Schuldenabbau beizutragen. Eher wollte die Konservative Regierung die Steuerquote senken. Im Verhältnis zu den anderen EU-Ländern liegen die wesentlichen Steuersätze relativ tief, und die Steuerquote ist unter allen EU-Mitgliedsländern außer Portugal am niedrigsten. ${ }^{412}$

Deswegen ist es bei der Beurteilung des steuerpolitischen Spielraums für Einnahmenerhöhungen anzunehmen, daß der Harmonisierungsdruck der EU noch keine Grenze der Besteuerung für das Vereinigte Königreich darstellt. Lediglich das in-

\footnotetext{
${ }^{410}$ Siehe z. B. Kay und Thompson (1986), S. 28ff, Yarrow (1986) und Djaroueh (1994).

411 Das Nettostaatsvermögen sank von $£ 99$ Mrd. 1979 auf $£ 45$ Mrd. 1992, Hills (1996), Tabelle 4.1.

412 Eurostat, (1995), S. 218. 
folge relativ hoher Verbrauchsteuern auf Alkohol und Tabak populäre grenzüberschreitende Einkaufen stellt durch die entsprechenden Einnahmeverluste eine gewisse Beschränkung dar. Die wesentlich niedrigeren Verbrauchsteuern auf Alkohol in Frankreich haben britische Konsumenten zu Einkaufsausflügen bewegt. Die resultierenden Steuerausfälle wurden 1993 auf $£ 600$ Millionen geschätzt, was circa 4-5\% der gesamten Einnahmen aus diesen Steuern ausmachte. ${ }^{413}$ Aber selbst für diese hoch besteuerten Güter scheint die Preiselastizität der Nachfrage insgesamt nicht so empfindlich zu sein, daß höhere Steuersätze eine Einnahmensenkung bedeuten würde. ${ }^{414}$

Eine zweite Grenze der Besteuerung wurde durch die Auslösung von Substitutionshandlungen festgestellt, wie sie die erweitertete Laffer-Kurve im zweiten Kapitel beschreibt. Die Diskussion zeigte auch, daß der kritische Steuersatz im Sinne der Laffer-Analyse sehr hoch liegt. Wie die offiziellen Berechnungen der Auswirkungen der Steueränderungen im jährlichen Finanzgesetz zeigen, hat die Regierung selbst auch keinen Aufkommensanstieg infolge der Steuersenkungen erwartet. ${ }^{415}$ Mit anderen Worten glaubte sie, daß die Einkommensteuersätze auf dem aufsteigenden Ast der Laffer-Kurve liegen und Steuersenkungen zu Einnahmeausfällen führten. Wenn budgetpolitische Überlegungen dies erforderlich machten, wurden deswegen die Tarifsenkungen durch andere einnahmenerhöhende Maßnahmen (Tariferhöhungen bei anderen Steuern oder eine Verbreiterung der Bemessungslagen) kompensiert. Insgesamt scheinen die Effizienzüberlegungen der Laffer-Analyse auch keine ökonomische Grenzen für die Steuereinnahmen im Vereinigten Königreich darzustellen.

In zwei Fällen sind eindeutige politische Grenzen der Besteuerung auszumachen. Bei der Einführung der kommunalen „Poll tax“ und der Erhebung der Mehrwertsteuer auf Brennstoffe für den Haushaltsverbrauch konnte die Konservative Regierung die gewünschten Steuererhöhungen aufgrund politischen Widerstands nicht durchsetzen. In beiden Fällen war die abrupte und regressive Wirkung der Steuerveränderung ausschlaggebend für den Widerstand. ${ }^{416}$ Die sonstigen Einkommenund Mehrwertsteuerreformen haben seit 1979 die Progressivität des Steuersystems eigentlich viel stärker reduziert, als die zwei mißlungenen Steuergesetze. ${ }^{417}$ Aber weil diese Veränderungen nur allmählich geschahen, lösten sie keine wirkungsvolle Opposition aus. Es gibt keine Hinweise dafür, daß politische Grenzen für die

413 Gaysford (1994), S. 24. Zum grenzüberschreitenden Einkaufen in der EU siehe auch Fitz Gerald (1990).

${ }^{414}$ Davies et al. (1994), Tabelle 6.12. sowie Crawford und Tanner (1995), S. 104.

415 Vgl. hierzu die jährlichen Berichte über die Haushaltspläne und ihre Auswirkungen in der November (bis 1992 März) Ausgabe von Economic Trends (CSO).

${ }^{416}$ Hierzu Smith, P. (1991), S. 434f, Fletcher (1992), S. 81ff, sowie Financial Times vom 10.12.94.

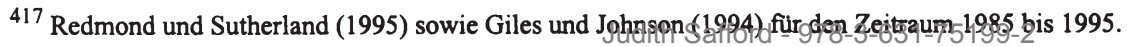


wesentlichen Steuern erreicht sind und eine Einnahmeerhöhung unmöglich machen. Trotzdem werden in der politischen Diskussion wesentliche Steuererhöhungen als ein politisches Tabu angesehen. ${ }^{418}$ Bislang hat die Konservative Regierung den seit Anfang der 90er Jahre erhöhten Finanzierungsbedarf nicht durch Steuern, sondern durch Neuverschuldung gedeckt (vgl. hierzu die Abbildungen 19 und 13).

\section{Die Entwicklung der Staatsausgaben}

\section{1) Wachsender Druck zu Mehrausgaben}

Das letzte Kapitel zeigt den Anstieg der Ausgabenquote unter der Konservativen Regierung auf, und wie die Ausgaben die Entwicklung der Staatsfinanzen entscheidend bestimmen. Der Ausgabenanstieg betraf nur bestimmte Ausgabenkategorien, und es ist anzunehmen, daß der Aufwärtstrend in diesen Bereichen in den nächsten Jahren fortbesteht. ${ }^{419}$

- Ein wesentlicher Teil der Einsparungen bei den Ausgaben in den 80er Jahren betraf die Investitionen. Allerdings wurde bereits nach 1976 bei den Investitionen gespart. Infolgedessen hat sich ein Investitionsbedarf angehäuft, welchem durch höhere Investitionsausgaben in der nächsten Zukunft entsprochen werden muß. ${ }^{420}$ Verzögerungen bei Instandhaltung, Reparatur- und Sanierungsarbeiten haben zu einer Verschlechterung des öffentlichen Kapitalstocks geführt (vgl. viertes Kapitel, Abschnitt III.2.a) und die Kosten der Instandsetzung ansteigen lassen, was die Ausgaben in der Zukunft zusätzlich belasten wird. ${ }^{421}$

- Eine mutmaßliche Zunahme der Kriminalität sowie eine härtere öffentliche Einstellung gegenüber Rechtsbrechern übt einen fortlaufenden Druck auf die Ausga-

${ }^{418}$ Vgl. hierzu The Independent, 2.9.96, S. 13, Economist, 23.11.96, S. 20.

419 So die Ansicht des britischen Schatzamts, siehe dazu Hibberd (1993a), S. 31. Siehe auch Dilnot (1995), S. 5 und Webb (1995), S. 12.

${ }^{420}$ Hibberd (1993b), S. 74.

${ }^{421}$ Siehe hierzu NEDC (1985). Beim Straßenunterhalt beispielsweise führt eine Unterlassung der minimalen Wartungsarbeiten alle vier bis acht Jahre zu einer Verzehnfachung des Kostenanstiegs, wenn aufgrund unterlassener Wartung der gesamte Belag erneuert werden muß. Wird auch die Erneuerung nicht rechtzeitig unternommen und das Fundament der Straße geschädigt, steigen die Kosten um das Fünfzigfache. Eine Befragung der Gemeinden 1984 im Rahmen der NEDC Studie ergab, daß eine minimale Wartung der Hauptstraßen durchschnittlich alle zehn bis zwölf Jahre stattfand, S. $6 \mathrm{f}$. 
ben für die innere Sicherheit aus. ${ }^{422}$ So werden beispielsweise der Bau von neuen Gefängnissen und die Aufstockung der Polizei zusätzliche Ausgaben erfordern.

- Bei sinkenden Schülerzahlen ist der Ausgabenanteil am BIP für den Bildungssektor im Untersuchungszeitraum relativ konstant geblieben. Trotzdem werden die Bildungsausgaben von den Wählern als unzureichend empfunden. ${ }^{423}$ Die Anforderungen an das Gut Bildung nehmen mit steigendem Einkommen überproportional $\mathrm{zu}$, was einen fortlaufenden Druck auf die Bildungsausgaben erwarten läßt.

- Die Gesundheitsausgaben sind ebenfalls einem starken Aufwärtsdruck ausgesetzt. Die Überalterung der Bevölkerung sowie technischer Fortschritt im Gesundheitswesen, welcher zu kostenspieligeren Behandlungsmethoden führt, sind zwei Gründe für steigende Kosten. Zudem wollen die Haushalte mit steigendem Einkommen auch relativ mehr Gesundheitsgüter und -leistungen konsumieren.

- Aus sozialen und demographischen Gründen wird in den nächsten Jahren eine steigende Anzahl von Empfängern staatlicher Unterstützung erwartet. Dadurch besteht ein starker Druck auf die Ausgaben für die soziale Sicherung. Die einzelnen Unterstützungszahlungen sind bereits auf ein sehr tiefes Niveau gedrückt worden, ${ }^{424}$ was weitere Einsparungen politisch kaum durchsetzbar macht.

Der im ersten Absatz dargestellte Investitionsbedarf entstand wohl deshalb, weil Einsparungen bei den Investitionen politisch am einfachsten durchzusetzen sind..$^{425}$ Die sonstige Entwicklung scheint die Wagnersche Hypothese des Strukturwandels zu unterstützen (2. Kapitel, Abschnitt IV.2.a). Gesellschaftliche Veränderungen haben die Nachfrage nach den Einrichtungen des Mindeststaates und den Vorsorgeleistungen des Wohlfahrtsstaats erhöht. Dazu kommt eine steigende Nachfrage nach einkommensabhängigen Gütern und Dienstleistungen.

Die zweite Wagnersche Hypothese findet in der Entwicklung aber auch Unterstützung (2. Kapitel, Abschnitt IV.2.b). Dieser zufolge widmet sich der Wohlfahrtsstaat vermehrt Umverteilungsaufgaben. Die höchsten Zuwachsraten erfuhren die Bereiche der sozialen Sicherung, der Gesundheit und - unter Berücksichtigung sinkender Anzahl von Schülern - der Bildung. Diese drei Ausgabenkategorien machten $197950 \%$ der Gesamtausgaben aus. 1994 ist der Anteil auf 62\% angestiegen.

\footnotetext{
422 Hierzu Brook und Cape (1995), insbesondere S. 204f.

${ }^{423}$ Skidelsky (1995), S. 61.

4241991 betrug beispielsweise das durchschnittliche Arbeitslosengeld 15,4\% vom durchschnittlichen Lohn zuzüglich Arbeitgeberversicherungsbeiträge. Zum Vergleich liegt der Anteil in Deutschland bei $34,3 \%$ und im OECD Durchschnitt bei 32,8\%, OECD (1993d), S. 44.
}

425 Mullard (1987), S. 173. 
Allen drei Kategorien ist gemeinsam, daß sie in erster Linie Verteilungscharakter haben. ${ }^{426}$

Die Analyse im 2. Kapitel über die Nachfrage nach Umverteilungsaufgaben zeigt, daß hinter einem Anstieg der Staatsausgaben mit Verteilungscharakter Interessengruppen stehen könnten (Abschnitt IV.2.c). Auch wenn dies in einzelnen Fällen vorgekommen sein mag, ist das staatliche Angebot in den Bildungs- und Gesundheitssektoren gerade dadurch gekennzeichnet, daß es im Lebenszyklus Nutzen für alle stiftet und nicht ausgesprochene Vorteile für spezielle Gruppen bietet. Dies gilt ebenso für die bedeutendsten Transferzahlungen. Die staatlichen Pensionszahlungen und das Kindergeld sind Pauschalzahlungen, welche in einem bestimmten Lebensabschnitt allen gleichermaßen zustehen. Die anderen wichtigen Transferzahlungen sind diejenigen für die Vor- und Fürsorgeleistungen des Staates, welche eine Art Versicherung für den einzelnen gegen die finanziellen Folgen von unvorhersehbaren Ereignissen, wie z. B. Krankheit, Unfall oder Erwerbslosigkeit, bietet. ${ }^{427}$ Diese Ausgaben für Transferzahlungen scheinen nicht eine Folge starker Interessengruppen zu sein, sondern ergeben sich aus der sozialen Verpflichtung des Staates, ein Existenzminimum für seine Bürger zu gewährleisten.

Die Analyse im zweiten Kapitel betont auch, daß die Ursache von steigenden Staatsausgaben auch beim Angebot liegen kann. Ein ,relativer Preiseffekt“ kann z. B. durch nutzenmaximierende Bürokraten oder X-Ineffizienzen entstehen (Vgl. 4. Kapitel, Abschnitt IV.3). Die Konservative Regierung hat den relativen Preiseffekt als einen wichtigen Bestimmungsgrund für die Höhe der Ausgaben betrachtet. Der Abbau des öffentlichen Dienstes und wettbewerbsstärkende Maßnahmen haben einen Beitrag dazu geleistet, die Kosten des öffentlichen Angebots zu senken. ${ }^{428}$

Zusammenfassend kann festgestellt werden, daß der Anstieg der Staatsausgaben im wesentlichen Güter und Dienstleistungen betraf, welche erstens Verteilungscharakter hatten und zweitens der Allgemeinheit zugute kamen. Bei den Transferzahlungen waren es entweder ebenfalls Überweisungen mit universalem Nutzen oder nach Bedürftigkeit zur Sicherung des Existenzminimums erteilte Transfers. Nach den Überlegungen des zweiten Kapitels kann für derartige Ausgaben die Streuung der Einkommensverteilung eine wichtige Rolle spielen (Abschnitt IV.2.b). Um dies

${ }^{426}$ Es gibt auch Effizienzargumente für die Bereitstellung von Bildung und Gesundheit, sowie die Zahlung von Transfers, welche durch Marktversagen begründet wird. Diese können aber das Ausmaß des Wohlfahrtsstaates nicht erklären, Dilnot (1995), S. $3 f$.

${ }^{427}$ Ein Teil dieser Risiken kann und wird im Vereinigten Königreich privat versichert. Allerdings versagt vor allem für Individuen, welche als hohe Risiken eingestuft werden, der Markt, Disney und Webb (1989), S. 55.

${ }^{428}$ OECD (1989), S. $63 \mathrm{ff}$. 
zu untersuchen, bedarf es einiger ausholender Erläuterungen, die im Exkurs des nächsten Abschnittes dargelegt werden.

\section{2) Exkurs: Der Einfluß der Einkommensverteilung auf die Ausgaben}

Als Grundlage ist es zunächst notwendig, die Entwicklung der Einkommensverteilung im Vereinigten Königreich seit 1979 kurz zu beschreiben. Danach wird dargelegt, wie diese Entwicklung mit dem Anstieg der Ausgaben, insbesondere derjenigen für Transfers, zusammenhängt.

\section{a) Die Entwicklung der Einkommensverteilung seit 1979}

Die Ungleichheit der Einkommensverteilung im Vereinigten Königreich nahm zwischen 1979 und 1992 rapide $\mathrm{zu}$ und erreichte seit dem Zweiten Weltkrieg das höchste Maß. ${ }^{429}$ Abbildung 29 gibt einen Eindruck der relativen Veränderungen.

\section{Abbildung 29: Anteile der Einkommensverteilung}

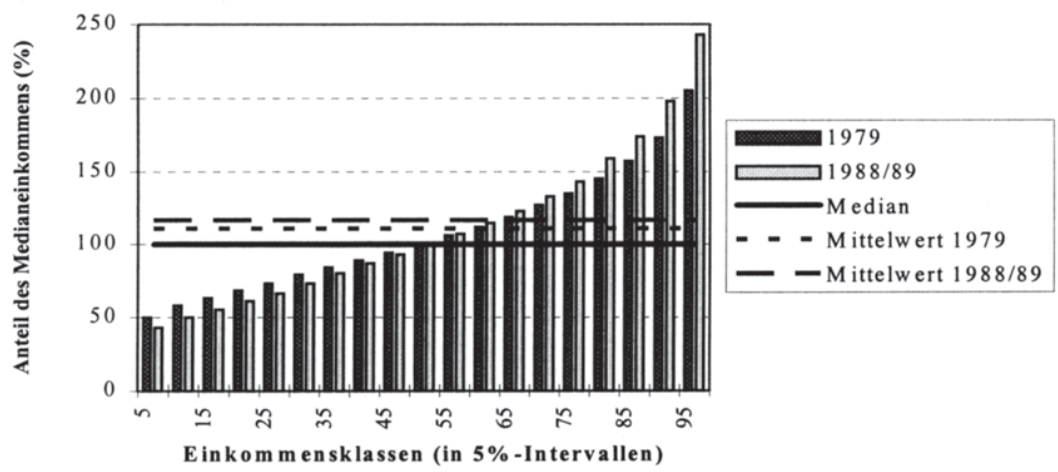

Anmerkung: Netto Einkommen (vor Wohnkosten)

Mittelwerte: $1979111=£ 167 /$ Woche; 1988/89 117=£202/ Woche (April 1992 Preise).

Quelle: Jenkins und Cowell (1994), Tabelle 1

${ }^{429}$ Letzte Erhebungen reichen bis spätestens 1992. Unter den neuen Untersuchungen siehe Atkinson (1993), Jenkins und Cowell (1993), Jenkins und Cowell (1994), Goodman und Webb (1994), Hills (1995a,b). 
Auf der Abszisse sind die jeweiligen Einkommensgruppen im Verhältnis zur Medianeinkommensgruppe (bei 50\%) eingetragen. Die Gruppen, welche weniger als das Medianeinkommen erhalten, sind links und in neun unterdurchschnittlichen Einkommensgruppen eingeordnet. Rechts von der Medianeinkommensgruppe liegen die neun überdurchschnittlichen Einkommensgruppen. Auf der Ordinate ist ihr jeweiliger Anteil am Medianeinkommen eingetragen.

Zwischen 1979 bis 1988/89 nahm beispielsweise das durchschnittliche Einkommen der ärmsten Gruppe der Einkommensbezieher relativ zum Medianeinkommen von $50 \%$ auf $43 \%$ ab. 1979 war das durchschnittliche Einkommen der höchsten Einkommensgruppe (oberste 5\%) viermal größer als dasjenige der niedrigsten Einkommensgruppe. Bis 1988/89 wuchs dieses Verhältnis auf 5,7 an.

Dieser Trend setzte sich auch während der Rezession Anfang der 90er Jahre fort. Zwischen 1979 und 1992 haben die ärmsten $20 \%$ bis 30\% der Bevölkerung praktisch keine reale Einkommensverbesserung erfahren. ${ }^{430}$ Die Abbildung 30 verdeutlicht diese Entwicklung bis 1988/89. Die Einkommensgruppen sind auch bei dieser Abbildung auf der Abszisse eingeteilt. Die Ordinate zeigt die Nettoeinkommensveränderungen. ${ }^{431}$ Die ärmsten fünf Prozent der Bevölkerung hatten 1988/89 einen Einkommensverlust von 2,4\% gegenüber 1979 zu verzeichnen. Dagegen nahm das Einkommen der reichsten Fünfprozent um $28,6 \%$ zu. Das durchschnittliche Einkommen (Mittelwert) nahm insgesamt um $27,5 \% \mathrm{zu} .{ }^{432}$

${ }^{430}$ Hills (1995a), S. 15, sowie Goodman und Webb (1994), S. 33 f.

${ }^{431}$ Unter Abzug der Wohnkosten (Mieten, Wasserabgaben, Netto-Hypothekarzinszahlungen, Versicherungsbeiträge, sonstige Abgaben). Im Vereinigten Königreich wird aufgrund der Struktur des Wohnungsmarkts (sehr hoher Eigentumsanteil, Mietbedarf im wesentlichen durch öffentliches Angebot abgedeckt) angenommen, $\mathrm{da} B$ vor allem Niedrigeinkommensbezieher wenig Einfluß auf ihre Wohnkosten ausüben können und diese deswegen eher die Eigenschaften von einer Steuer haben als von Ausgaben. Deswegen wird für manche verteilungspolitische Statistiken das Nettoeinkommen abzüglich der Wohnkosten berechnet. Allerdings verstärkt sich dadurch die Ungleichmäßigkeit der Einkommensverteilung nur gering, Jenkins und Cowell (1993), S. 3. Zur Diskussion siehe Johnson und Webb (1992).

432 Jenkins und Cowell (1994), S. $110 \mathrm{f}$. 


\section{Abbildung 30: Durchschnittliche Einkommensverănderungen zwischen 1979 und 1988/89 nach Einkommensgruppen}

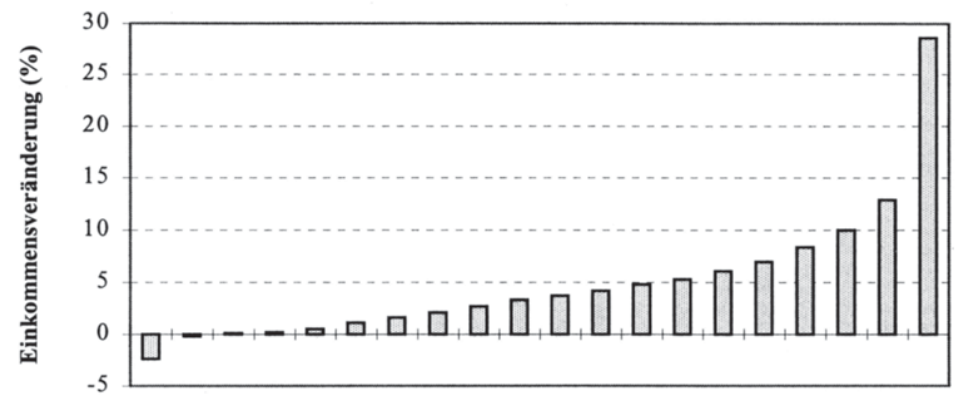

Einkommensgruppe (zwanzigstel)

Quelle: Jenkins und Cowell (1993), Tabelle 4

\section{b) Folgen der Konservativen Politik auf die Einkommensverteilung ${ }^{433}$}

Die Gründe für diese Veränderung waren erstens, wie bereits im 4. Kapitel festgestellt, eine absolute Zunahme der Empfänger von staatlichen Unterstützungszahlungen, und zweitens eine starke Zunahme der Einkommensunterschiede zwischen Beschäftigten und Transferempfängern. Zwischen 1979 und 1994 sind die Transferleistungen relativ zu den Löhnen gesunken. Dieser Effekt trat besonders stark beim wachstumsbedingten Anstieg der Reallöhne zwischen 1984 und 1990 auf, weil die Unterstützungszahlungen seit Anfang der 80er Jahre entweder, wie das Arbeitslosengeld, zeitweise eingefroren, oder, wie die Pensionszahlungen, lediglich der Inflation angepaßt wurden (vgl. 3. Kapitel, Abschnitt IV.3.b). Das Ausmaß dieser Entwicklung wird deutlich, wenn die Änderungen der Ungleichheitsmaße der Primär- und Bruttoeinkommensverteilungen (d. h. einschließlich Transferzahlungen, aber vor Steuern) in den $80 \mathrm{er}$ Jahren miteinander verglichen werden. Von 1984 bis 1990 stieg der Gini-Koeffizient der Primärverteilung um vier Prozentpunkte von $49 \%$ auf $53 \%$ an. In der gleichen Periode stieg der Koeffizient für das Bruttoeinkommen um sieben Prozentpunkte. Mit anderen Worten, die Transferleistungen wirkten der Ungleichheit der Primäreinkommensverteilung nicht mehr entgegen, sondern verstärkten diese noch. ${ }^{434}$

${ }^{433}$ Hierzu Johnson und Webb (1993) und Hills (1995b), S. 38ff.

${ }^{434}$ Hierzu Hills (1995a), S. 25f. und Abbildung 11. 
Drittens haben zunehmende Lohndifferentiale infolge arbeitsmarktpolitischer Reformen $^{435}$ die Ungleichmäßigkeit der Einkommensverteilung unter den Beschäftigten erhöht. Ein zunehmender Anteil der Niedriglohnverdiener erhalten staatliche Unterstützungszahlungen, weil ihre Einkünfte das Existenzminimum nicht abdekken. Ein Beispiel dafür ist die nach Bedürftigkeit gestaffelte Familienunterstützung (Family Credit) für beschäftigte Familien mit Kindern (vgl. 4. Kapitel, Abbildung 26, S. 150). 1994/95 erhielten fast 600.000 Familien im Durchschnitt $£ 48 /$ Woche von dieser Zuwendung. Die Regierung plant auch die Einführung einer neuen $\mathrm{Zu}-$ wendung namens „Earnings Top Up“, welche ähnlich wie der „Family Credit“, jedoch an kinderlose Haushalte gewährt wird. ${ }^{436}$

\section{3) Steigende Staatsausgaben für Umverteilung}

Gemessen an der relativen Definition der Armut, wie sie in der Ökonomie und anderen Sozialwissenschaften üblich ist, ${ }^{437}$ hat die Entwicklung der Einkommensverteilung zu einer Zunahme der Bedürftigkeit geführt. Dem wird automatisch mit erhöhten Transferzahlungen begegnet, vorausgesetzt, daß die Höhe der Unterstützungszahlungen sich ebenfalls am relativen Armutsbegriff orientiert, indem beispielsweise der durchschnittliche Lohn als Bemessungsgrundlage für die Bedürftigkeit herangezogen wird. Die Konservative Strategie der Inflationsanpassung von Transferzahlungen basiert jedoch auf einem absoluten Armutskonzept, ${ }^{438}$ weil dadurch nur gerade der absolute Wert der Unterstützung erhalten bleibt (vgl. 4. Kapitel, Abschnitt III.3.b). Deswegen hat sich die Kluft zwischen Beschäftigten und Transferempfängern vergrößert.

Bei der Analyse dieser Strategie und ihrer möglichen Entwicklung können die Überlegungen von Meltzer und Richard (1981) herangezogen werden (vgl. 2. Kapitel, Abschnitt IV.2.b). Im Modell bedeutet die Einkommensabhängigkeit der Transferzahlungen, daß diese sich im Zeitverlauf proportional zur Einkommensentwicklung verhalten, d. h. sie unterstellt implizit einen relativen Armutsbegriff. Darüber hinaus nimmt bei zunehmender Ungleichmäßigkeit der Einkommensverteilung die Umverteilung in Form von Erhöhungen der Transferzahlungen über den Wahlprozeß noch überproportional zu.

\footnotetext{
${ }^{435}$ Hierzu siehe z. B. Hutschinson und Church (1989), S. 160f.

436 Webb (1995), S. $18 f$.

${ }^{437}$ Ein relatives Konzept von Armut bezieht sich auf das Wohlstandsniveau, gemessen beispielsweise am durchschnittlichen Einkommen, siehe z. B. Aktinson (1983), S. $237 \mathrm{ff}$.

${ }^{438}$ Diese Ansicht ist auch Taylor-Goodby (1995), S. 7 Judith Safford - 978-3-631-75199-2 
Zunehmende Ungleichmäßigkeit der Einkommensverteilung kommt im Modell von Meltzer und Richard (1981) durch einen größeren Abstand zwischen Mittelwertund Medianeinkommen zum Ausdruck, bzw. durch einen größeren Anteil des gesamten Einkommens, der über dem Medianeinkommen liegt. Wie die Abbildung 30 zeigt, ist diese Entwicklung im Vereinigten Königreich eingetreten. Entsprechend den Kriterien des Modells hätte deswegen die Umverteilung durch Transferzahlungen in den $80 \mathrm{er}$ Jahren zunehmen müssen. Wie oben gezeigt wurde, ist zwischen 1984 und 1990 genau das Gegenteil geschehen. Erstens bewirkte die Abkoppelung der Transferzahlungen von der Einkommensentwicklung, da $\beta$ in Wachstumsperioden wie 1984 bis 1990, die Transferzahlungen die Veränderung der Primärverteilung nicht kompensierte. Zweitens wurde die Konservative Regierung nicht abgewählt, bzw. nicht gezwungen, für ihre Wiederwahl diese Politik zu verändern.

Bei der erwarteten Zunahme der Anzahl der Transferempfänger stellt sich jedoch die Frage, ob die bisher verfolgte Strategie fortgesetzt, und die Auswirkung einer zunehmenden $\mathrm{Zahl}$ von Berechtigten durch sinkende relative Leistungen zumindest teilweise absorbiert, oder sogar langfristig ein Anstieg der Transferzahlungen verhindert werden kann. Wird aus der Sicht der Wähler das Einfrieren des Lebensstandards für Leistungsabhängige auf das Niveau von 1980 politisch akzeptierbar sein, wenn die Anzahl von Leistungsabhängigen weiterhin zunimmt und beispielsweise die Lebensstandards anderer Gesellschaftsgruppen sich verdoppelt haben? Wird der von Meltzer und Richard (1981) postulierte Verteilungsmechanismus langfristig doch zum Tragen kommen, und werden die Wähler Erhöhungen der Transferzahlungen durchsetzen?

Wie ist im Hinblick auf das langfristige Ziel einer Senkung der Staatsquote diese Politik zu beurteilen? Nach Meltzer und Richard (1981) müßte eine Koalition der relativ ärmeren Gesellschaftsmitglieder bis hin zum Medianwähler, (d. h. knapp über $50 \%$ der Wähler) für höhere Transferzahlungen sein. Dies scheint jedoch nicht der Fall zu sein. Nach Wählerumfragen befürwortet zwar ein Großteil der Bevölkerung Staatsausgaben zur Behebung der Armut. Der Anteil von Befürwortern lag aber bereits 1979 bei $83,5 \%$ - weit über $50 \%$ ! - und stieg bis 1987 nur geringfügig auf $87,9 \%$ an. Diese Zunahme ist zudem nicht auf den erhöhten Anteil an Transferempfängern zurückzuführen. Eine Aufteilung der Befragten in drei Einkommensklassen zeigte nämlich, daß diese sich in ihren Wünschen nur geringfügig voneinander unterschieden. ${ }^{439}$

Möglicherweise haben negative Externalitäten der zunehmenden Armut die NichtEmpfänger zur Unterstützung von staatlichen Ausgaben zur Armutslinderung bewegt. Unsicherheit unter den mittleren Einkommensklassen könnte die Unterstüt-

${ }^{439}$ Crewe, I. et al. (1991), Tabellen 10.19 und 10.26. 
zung für erhöhte Transferzahlungen von dieser Seite ebenfalls stärken. ${ }^{440}$ Denn wenn Beschäftigung keine Garantie mehr für das Existenzminimum darstellt und Beschäftigte zunehmend auf staatliche Unterstützung angewiesen sind, steigt nicht nur die objektive Last der Transferzahlungen, sondern auch die gesellschaftliche Empfindsamkeit für staatliche Verteilungsmaßnahmen an. Daraus kann sich ein zusätzlicher Druck auf andere Ausgaben, wie z. B. für Gesundheit und Bildung, entwickeln.

Wenn fast $90 \%$ der Wähler staatliche Maßnahmen zur Behebung der Armut wünschen, so ergibt sich die Frage wie sich dies im Hinblick auf konkrete Maßnahmen äußert. Weitere Umfragen in bezug auf die einzelnen Ausgabenkategorien zeigen, daß heute die Wähler höhere Transferleistungen wollen: waren im Jahre 1979 nach Meinung von $50,1 \%$ der befragten Wähler die Sozialehilfeleistungen zu großzügig und nur für $17 \%$ nicht genügend, waren 1987 nur noch 24,8\% für einen Abbau, wogegen sich $34,9 \%$ für höhere Sozialhilfeleistungen aussprachen. ${ }^{441}$ Und 1993 befürworteten $78 \%$ der Befragten höhere Ausgaben für Pensionszahlungen und $48 \%$ höheres Arbeitslosengeld. ${ }^{442}$ Diese Ergebnisse müssen jedoch aus zwei Gründen relativiert werden. Erstens, wenn die Wähler gleichzeitig mit ihrer Befürwortung höherer Transferzahlungen ihre Bereitschaft zu Steuererhöhungen erklären müssen, sinkt die Unterstützung für Ausgabenerhöhungen. ${ }^{443}$ Zweitens, werden die Prioritäten für Transferzahlungen im Vergleich mit anderen Ausgaben als eher tief eingestuft. So gab 1994 die überwiegende Mehrheit der Wähler die erste oder zweite Priorität dem Gesundheits- (72\%), Bildungs- (60\%) oder Wohnungssektor (19\%). Lediglich $12 \%$ der 3.500 Befragten räumte Ausgaben für die soziale Sicherung eine vergleichbar hohe Priorität ein. Die Prioritätensetzung wich zudem bei der niedrigsten Einkommensgruppe kaum von der Grundgesamtheit ab: Für Gesundheit lag dort die Unterstützung bei $67 \%$, für Bildung bei $57 \%$ und für Wohnungspolitik und soziale Sicherung bei jeweils $21 \% .{ }^{444}$

Die Meltzer/Richard-Hypothese, daß die ärmeren Gesellschaftsmitglieder für höhere Transferleistungen stimmen, wird durch diese Ergebnisse nicht unterstützt. Die Staatsausgaben für die allgemeinen meritorischen Güter wie Gesundheit und Bildung scheinen auch ihnen wichtiger als das Niveau der Unterstützungszahlungen zu sein. Deswegen ist es sehr schwer für die Konservative Regierung, die Ausga-

\footnotetext{
${ }^{440}$ So die These von Hutton (1995).

441 Frage zur Armut: 19791.175 und 19873.711 Befragte; Frage zur Sozialhilfe: 19791.812 und 1987 3.643 Befragten, siehe Crewe, I. et al. (1991), Tabelle 10.19.

442 Es wurden circa 3.500 Personen befragt. Lipsey (1994), Tabelle 1.

443 Skidelsky, (1995). Immerhin erklärten sich 1993 63\% der Befragten bereit, für mehr Ausgaben für Bildung, Gesundheit und soziale Sicherung Steuererhöhungen in Kauf zu nehmen. 1983 waren es nur 32\%, Taylor-Gooby (1995), S. 3.

444 Taylor-Gooby (1995), S. 4ff. 
ben in den Bereichen Gesundheit und Bildung zu senken. Solange andererseits noch fast $90 \%$ der Befragten die Aufhebung der Armut als staatliche Aufgabe sehen, wird die soziale Sicherung ebenfalls eine bedeutende Ausgabenkategorie bleiben müssen.

\section{Die Finanzierung zusätzlicher Staatsausgaben}

Wenn der Druck auf die Ausgaben für Gesundheit, Bildung und soziale Sicherung in den nächsten Jahren voraussichtlich nicht abnimmt, muß die Frage gestellt werden, wie diese Ausgaben zu finanzieren sein werden (vgl. hierzu 1. Kapitel, Abschnitt II.2.f). Aus der Diskussion im Abschnitt I dieses Kapitel wurde deutlich, daß unter diesen Finanzierungsquellen lediglich die Steuern in Frage kommen. Diese Option möchte jedoch die Konservative Regierung auf alle Fälle vermeiden. Deswegen muß nach alternativen Finanzierungskonzepten gesucht werden.

\section{1) Marktorientierte Mechanismen}

Eine zumindest partielle Lösung der Finanzierungsfrage ist es, die Bereitstellung von Leistungen und Gütern von staatlichen auf private Anbieter zu übertragen und sie so aus dem staatlichen Sektor auszugliedern. Bildung und Gesundheit sind meritorische Güter, und deswegen könnte der private Markt zumindest einen Teil der wachsenden Nachfrage befriedigen. ${ }^{445}$ Selbst bei öffentlichen Gütern und Leistungen kann die Bereitstellung häufig durch Private gewährleistet werden. Wie bereits diskutiert wurde, wird diese Strategie auch im Vereinigten Königreich verfolgt (4. Kapitel, Abschnitt III.3.b), und ihr weiterer Ausbau ist von der Regierung geplant. ${ }^{446}$

Eine Dezentralisierung von distributiven Ausgaben wie beispielsweise Gesundheit und Bildung könnte durch die Ausbildung von stärkeren lokalen Strukturen alternative staatliche Angebotsquellen in konkurrierenden Gebieten schaffen (2. Kapitel, Abschnitt IV.4). Zudem sind Effizienzgewinne in einer lokalen Körperschaft durch administrative und informationelle Kostenersparnisse im Sinne von Coase

\footnotetext{
${ }^{445}$ Für Konzepte hierzu siehe z. B. Skidelsky (1995) sowie den von Seldon (1996) herausgegebenen Sammelband.

${ }^{446}$ Siehe hier auch Seldon (1996). 
(1937) möglich. ${ }^{447}$ Auswirkungen von Dezentralisierung auf die Staatsausgaben und ihre Finanzierung sind ein breites und umstrittenes Thema, das bisher unter Berücksichtigung politischer Rahmenbedingungen wenig erforscht wurde. Im Rahmen dieser Abhandlung kann es deshalb hier nur am Rande erwähnt werden. ${ }^{448}$ Ohnehin hat die Konservative Regierung bis jetzt eine Strategie der Zentralisierung staatlicher Aufgaben und ihrer Finanzierung verfolgt (vgl. Abbildung 17, sowie 4. Kapitel, Abschnitt III.3.b), so daß eine Dezentralisierungspolitik von ihr auch zukünftig kaum zu erwarten sein wird.

Wettbewerbsorientierte Lösungen stoßen bei den Ausgaben für soziale Sicherung an Grenzen. Eine Verteilung von den „Reichen“ zu den „Armen“, wie es die meisten Ausgaben für soziale Sicherung letztlich darstellen, kommt auf privaten Märkten nicht freiwillig zustande. ${ }^{449}$ Migration im Sinne von Tiebout (1956) schränkt die Anwendung von lokaler Umverteilungspolitik ein. ${ }^{450}$ Derartige Umverteilungen, sowie ihre Finanzierung, liegt grundsätzlich in zentralstaatlicher Verantwortung. ${ }^{451}$

\section{2) Finanzierung durch Staatsverschuldung}

Die kurzfristig einfachste Lösung der Finanzierungsfrage ist eine staatliche Kreditaufnahme. Beurteilt nach der Entwicklung der Staatsverschuldung seit 1991, hat sich die Konservative Regierung dieser Strategie bedient und dabei einen rasanten Anstieg der Staatsverschuldung von bisher unbekannten Ausmaß während einem wirtschaftlichen Aufschwung und in einer Friedenszeit herbeigeführt.

Eine Erklärung für diese Entwicklung bietet die Diskussion der Politökonomie der Staatsverschuldung im ersten Kapitel (Abschnitt V.4). Cukierman und Meltzer (1989) zeigten Bedingungen auf, unter denen eine zunehmende Ungleichmäßigkeit der Einkommens- und Vermögensverteilung an eine erhöhte Bereitschaft zur öffentlichen Schuldenaufnahme begünstigt. Erstens führt die Verteilungsveränderung zu einem erhöhten Bevölkerungsanteil an liquiditätsbeschränkten Transferempfängern. Wie die Ausführungen im Abschnitt II.2.a zeigten, ist dies in den 80er Jahren der Fall gewesen.

\footnotetext{
${ }^{447}$ Helm und Smith (1987), S. ixf.

${ }^{448}$ Neuere Beiträge sind Persson und Tabellini (1993), Eichengreen und von Hagen (1996).

${ }^{449}$ Adverse Selektion und „moral hazard“ führen dazu, daß ein privater Versicherungsmarkt für viele Für- und Vorsorgerisiken versagt, Jackson (1996), S. 6 f.

${ }^{450}$ Helm und Smith (1987), S. viif.

${ }^{451}$ Dilnot (1995), S. 10. 
Zweitens werden die Präferenzen für Staatsverschuldung verstärkt, wenn derjenige Gesellschaftsanteil zunimmt, welcher sein Einkommen aus Kapitalerträgen bezieht. Jenkins (1995) fand, daß die Verteilung von Erträgen aus Vermögen zwischen 1981 und 1986 ungleichmäßig geworden ist und die Korrelation zwischen Kapitalerträgen und anderen Einkommensquellen zunahm. Ryan (1996) fand bei einer Untersuchung der funktionellen Verteilungsentwicklung, daß Einkünfte aus Kapital zugenommen haben. Insgesamt machten in der zweiten Hälfte der 80er Jahre Kapitaleinkommen einen wichtigen Beitrag zur gesamten Ungleichheitsentwicklung aus. ${ }^{452}$ Zudem gibt es zahlreiche anekdotische Hinweise für die relative Stärke der Kapitalinteressen im Vereinigten Königreich. Bereits Keynes $(1922,1923)$ machte auf die Stärke der Interessen von britischen Kapitalbesitzern im Hinblick auf die Verschuldungsneigung kritisch aufmerksam. Die im Vereinigten Königreich einzigartige Verbindung zwischen dem Londoner Finanzmarkt einerseits und der Regierung und ihren Bediensteten andererseits, ${ }^{453}$ schafft nach Ingham (1984) eine Präferenz für die Interessen der Kapitalempfänger vor allem bei Konservativen Regierungen. Dies kann zu erhöhter Verschuldung führen. ${ }^{454}$

Drittens werden nach Cukierman und Meltzer (1989) die Präferenzen für Staatsverschuldung im Wachstumsprozeß verstärkt, wenn aufgrund von arbeitssparendem technischem Fortschritt die Reallöhne sinken und infolgedessen der Anteil von liquiditätsbeschränkten Individuen sich erhöht. Eine Senkung der Reallöhne für die Niedriglohnempfänger und ein entsprechender Anstieg der Transferzahlungen wurde im Vereinigten Königreich festgestellt (Abschnitt II.2.b). ${ }^{455}$ Daraus ist eine erhöhte Bereitschaft für Staatsverschuldung ebenfalls zu erwarten.

Schließlich nimmt die Wahrscheinlichkeit, liquiditätsbeschränkt zu sein, mit steigendem Alter zu, weil Konsum relativ wichtiger als Sparen wird. Deswegen führt nach Cukierman und Meltzer (1989) ein höherer Anteil von älteren Gesellschaftsmitgliedern zu einer erhöhten Verschuldungsneigung. Dieser Effekt wird selbstverständlich komprimiert, wenn die staatlichen Pensionszahlungen relativ zum Lohnniveau gekürzt werden.

Alle diese Bedingungen scheinen nach Cukierman und Meltzer (1989) den Umständen im Vereinigten Königreich zu entsprechen und bieten eine Erklärung da-

\footnotetext{
452 So auch das Ergebnis von Goodman und Webb (1994), S. 65.

453 Diese Beziehung geht zurück bis in das 17. Jahrhundert, als Londoner Finanziers, welche bereits die britische Politik und das Geschäftsleben dominierten, 1694 die Bank of England gründeten, Plumb (1956), S. 22

${ }^{454}$ Alesina (1988), S. 40, sowie Aghion und Bolton (1989).

455 Diese Entwicklung läßt sich allerdings nicht eindeutig auf den Wachstumsprozeß zurückführen. Sicherlich spielten Arbeitsmarktreformen, wie z. B. die Abschaffung von Mindestlöhnen, eine wichtige Rolle. 
für, warum hauptsächlich die Staatsverschuldung zur Finanzierung der steigenden Ausgaben gewählt wurde.

\section{Ausblick}

In dieser Arbeit wurde die Entwicklung der Staatsverschuldung seit 1979 bis 1994 aufgezeigt. Es wurde deutlich, daß die Konsolidierung der Staatsfinanzen in den 80er Jahren größtenteils auf einmaligen Einnahmen und dem Verteilungsmechanismus der Inflation beruhte. Ein stetiger Druck auf die Ausgaben konnte trotz einiger Reformen auf der Angebotsseite gegen die Wählermehrheit nicht durchgesetzt werden. Seit dem Wegfall der oben erwähnten Finanzierungsquellen Anfang der 90er Jahre wird erneut auf die Kreditfinanzierung zurückgegriffen.

Im März 1996, am Ende des Finanzjahres 1995/96, lag die Brutto-Verschuldung des öffentlichen Sektors im Vereinigten Königreich bei 51,8\%. ${ }^{456}$ Gegenüber 1979 ist dies immer noch 4,7\% tiefer. Allerdings macht der Anstieg innerhalb von vier Jahren vom Tiefpunkt im März 1991 bei 34,3\% zum heutigen Wert von fast $52 \%$ deutlich, wie schnell sich die öffentlichen Finanzen in den 90er Jahren verschlechtert haben.

Die Regierung ist jedoch immer noch nicht bereit, die für die Stabilisierung der Schuldenquote notwendigen Steuererhöhungen durchzuführen. Im Finanzgesetz vom November 1996 wurde sogar eine weitere Senkung des Einkommensteuersatzes um ein Prozent beschlossen. ${ }^{457}$ Deshalb wären aus einer langfristigen Perspektive Ausgabensenkungen dringend notwendig. Der Haushaltsplan sieht jedoch im Rahmen der NCT eine weitere Erhöhung der Ausgaben im Gesundheitssektor vor. Für die exogenen Komponenten der Zins- und Transferzahlungen wird ebenfalls ein Anstieg erwartet. Nach allgemeinem Konsens waren diese ökonomisch gesehen widersinnigen finanzpolitischen Entscheidungen als kurzfristige Reaktion der auf Wiederwahl hoffenden Regierung zu werten. ${ }^{458}$ Da Wahlen im Mai 1997 stattfinden mußten, war dieser Druck dannzumal besonders stark.

Für die langfristige Entwicklung der Staatsverschuldung im Vereinigten Königreich wird die Zunahme der Ungleichheit der Einkommensverteilung seit Anfang der 80er Jahre die Durchsetzung der notwendigen Reformen bei den Ausgaben und

\footnotetext{
${ }^{456}$ Bank of England, (1996), S. 427.

${ }^{457}$ Hierzu Economist von 30.11.96, S. 39 f.

${ }^{458}$ Economist von 23.11.96, S. 22.
} 
ihrer Finanzierung besonders schwierig machen. Eine ungleichmäßige Einkommensverteilung erhöht nicht nur in direkter Weise die Transferzahlungen, sondern scheint auch den politischen Druck der Wählerschaft für sonstige Ausgaben mit Verteilungscharakter zu verstärken.

Langfristig könnten viele Staatsausgaben mit Verteilungscharakter größtenteils dem privaten Sektor anvertraut werden. Auf den ersten Blick schaffen derartige Reformen eine Entlastung der Staatsfinanzen. Dabei blieben für den Staat fast nur noch die Transferzahlungen übrig und seine Rolle entwickelte sich immer stärker $\mathrm{zu}$ einem reinen Umverteilungsorgan hin. Es ist vielleicht möglich, dabei Kosten der staatlichen Aktivitäten zu sparen, aber wahrscheinlich wird der Finanzierungsdruck dadurch nicht gemindert. Wenn die Wohlhabenden in einer Gesellschaft ihre Gesundheit, Renten und Bildungsausgaben selber finanzieren müssen, werden sie dadurch den Ertrag ihrer Steuerzahlungen immer kleiner einschätzen, und dann wird die Steuerfinanzierung der restlichen Umverteilungsausgaben politisch noch schwieriger durchzusetzen sein.

Somit verbleibt für die Finanzierung der Transferzahlungen als Ausweg die Staatsverschuldung. Wie bereits im Rahmen der Diskussion des Ricardianischen Äquivalenztheorems (1. Kapitel, Abschnitt II.2), sowie beim Modell von Cukierman und Meltzer (1989) gezeigt wurde, ziehen die ärmeren und reicheren Mitglieder einer Gesellschaft die Staatsverschuldung sowieso als Finanzierung vor oder stehen ihr gleichgültig gegenüber. In der einkommensmäßig stark gespaltenen Gesellschaft des Vereinigten Königreichs sind deshalb die Bedingungen für eine weitere Erhöhung der Staatsverschuldung vorhanden. 


\section{Anhang}

\section{Crowding Out in IS-LM Modell (1. Kapitel, Abschnitt III)}

\section{1) Das kurzfristige IS-LM Modell}

Das gegebene Geldangebot wird zu Spekulations- und Transaktionszwecken nachgefragt:

$$
\text { LM: } \quad \frac{\mathrm{M}}{\mathrm{P}}=\mathrm{L}(\mathrm{r}, \mathrm{Y}), \text { mit } \mathrm{L}_{\mathrm{r}}<0 \text { und } \mathrm{L}_{\mathrm{Y}}>0 .{ }^{459}
$$

Die IS-Kurve gibt das Gütermarktgleichgewicht wieder:

IS: $\quad \mathrm{Y}=\mathrm{C}[\mathrm{Y}-\mathrm{T}(\mathrm{Y})]+\mathrm{I}(\mathrm{r})+\mathrm{G}$, mit $0>\mathrm{C}_{\mathrm{Y}}<1, \mathrm{~T}^{\prime}<1$ und $\mathrm{I}_{\mathrm{r}}<0$.

Die aggregierte Nachfrage setzt sich aus dem vom verfügbaren Einkommen abhängigen Konsum, den zinsabhängigen Investitionen und den Staatsausgaben zusammen. Die Betrachtung geht von einer Differentialanalyse aus. ${ }^{460}$ Bei gleichbleibenden Ausgaben substituiert der Staat die Erhebung von Steuern heute durch eine Schuldaufnahme. Wenn die Steuerfunktion die Form $\mathrm{T}(\mathrm{Y})=\tau \mathrm{Y}$ hat, lautet der Multiplikator für eine Senkung des proportionalen Grenzsteuersatzes von $\tau_{0} \mathrm{Y}$ auf $\tau_{1} \mathrm{Y}$ bei Konstanz der Geldmenge und der Staatsausgaben: ${ }^{461}$

${ }^{459}$ Die Schreibweise $Z_{x}$ bedeutet $\partial Z / \partial x$.

${ }^{460}$ Die Annahme geht auf Musgrave und Musgrave (1969) zurück, S. 158f.

${ }^{461}$ Die komparativ-statische Analyse des Systems läßt sich mit Hilfe der Jacobi-Determinanten Rechnung lösen. Nach Differenzieren der IS-LM Gleichungen

$$
: d Y=C_{Y}\left(d Y-\tau_{1} d Y-Y d \tau\right)+I_{r} d r \text { sowie } d\left(\frac{M}{P}\right)=L_{r} d r+L_{Y} d Y=0
$$

läßt sich das zwei-Gleichungen-System in Matrixform darstellen und eine endogene variable Jacobi-Determinante bilden:

$$
\left(\begin{array}{cc}
{\left[1-C_{Y}\left(1-\tau_{1}\right)\right]} & -I_{r} \\
L_{Y} & L_{r}
\end{array}\right)\left(\begin{array}{c}
d Y \\
d r
\end{array}\right)=\left(\begin{array}{c}
-C_{Y} Y d \tau \\
d M / P
\end{array}\right) .
$$

Die Jacobi Determinate $|\mathrm{J}|=\left[1-\mathrm{C}_{\mathrm{Y}}\left(1-\tau_{1}\right)\right] \mathrm{L}_{\mathrm{r}}+\mathrm{I}_{\mathrm{r}} \mathrm{L}_{\mathrm{Y}}<0$.

Anhand der Cramer'schen Regel kann die Lösung für die exogene Größe $\mathrm{d} \tau \mathrm{mit} d(\mathrm{M} / \mathrm{P})=0$ berechnet werden 


$$
\frac{d Y}{d \tau}=\frac{-C_{Y} Y}{\left[1-C_{Y}\left(1-\tau_{1}\right)\right]+\frac{I_{r} L_{Y}}{L_{r}}} .
$$

Die Auswirkungen der Steuersenkung lassen sich in drei Komponenten unterteilen. Im Nenner gibt der Ausdruck [1- $\left.\mathrm{C}_{\mathrm{Y}}\left(1-\tau_{1}\right)\right]$ die expansive Nachfragewirkung auf das verfügbare Einkommen beim neuen Grenzsteuersatz $\tau_{1}$ wieder. Im Zähler stellt $\mathrm{C}_{\mathrm{Y}} \mathrm{Y}$ die durch die Steuersenkung $d \tau$ induzierte Konsumänderung dar. Schließlich mißt $\mathrm{I}_{\mathrm{r}} \mathrm{L}_{\mathrm{Y}} / \mathrm{L}_{\mathrm{r}}$ das Ausmaß des „Crowding Outs“. Die Elastizitätseigenschaften der LM-Kurve ( $\mathrm{L}_{\mathrm{Y}} / \mathrm{L}_{\mathrm{r}}$ ist die Steigung der LM-Kurve), sowie die Zinselastizität der Investitionen ( $I_{r}$ beeinflußt die Steigung der IS Kurve) schränken die expansive Einkommenswirkung der Steuersenkung ein. Zum einem wird das Geldmarktgleichgewicht erhalten durch die entgegenwirkenden Kräfte des positiven Einkommenseffektes $\left(\mathrm{L}_{\mathrm{Y}}\right)$ und des negativen Portfolioeffektes $\left(\mathrm{L}_{\mathrm{r}}\right)$ auf die Kassenhaltung. Zum anderen wird einen negativen Zinseffekt auf die Investitionen postuliert. Je nachdem wie diese Determinanten Bedeutung erlangen, wird das Ausmaß an „Crowding Out" unterschiedlich auffallen. Grundsätzlich gilt: Je größer der Ausdruck $\mathrm{I}_{\mathrm{r}} \mathrm{L}_{\mathrm{Y}} / \mathrm{L}_{\mathrm{r}}$, desto geringer der Multiplikator.

\section{2) Das klassische Modell}

Mit einer vollkommen zinsunelastischen Geldnachfrage $\left(\mathrm{L}_{\mathrm{r}}=0\right)$ geht der Ausdruck $\mathrm{I}_{\mathrm{r}} \mathrm{L}_{\mathrm{Y}} / \mathrm{L}_{\mathrm{r}}$ im kurzfristigen Multiplikator gegen $\infty$ und die expansive Politik wird vollständigt verdrängt.

\section{3) Das langfristige IS-LM Modell}

Das bestandserweitete Gütermarktgleichgewicht ist

IS: $\quad Y=C[Y+B-T(Y+B) ; M+B / r+K]+I(r, K, Y)+G$, mit $0>\mathrm{C}_{\mathrm{Y}}<1, \mathrm{C}_{\mathrm{w}}>0, \mathrm{~T}^{\prime}<1$ und $\mathrm{I}_{\mathrm{r}}<0, \mathrm{I}_{\mathrm{K}}<0, \mathrm{I}_{\mathrm{Y}}>0$.

$$
\frac{d Y}{d \tau}=\frac{\left(\begin{array}{cc}
-C_{Y} Y & -I_{r} \\
0 & L_{r}
\end{array}\right)}{|J|}=\frac{\left(-C_{Y} Y\right) L_{r}}{\left[1-C_{Y}\left(1_{d i t} \tau_{1}\right) L_{L_{f f}}+I_{r} L_{978}\right.}<0
$$


Der Konsum hängt vom verfügbaren Einkommen einschließlich der Zinszahlungen auf die Staatsschuld sowie vom realen Vermögen $(\mathrm{W}=\mathrm{M}+\mathrm{B} / \mathrm{r}+\mathrm{K})$ ab. $\mathrm{M}$ ist die Geldmenge und $\mathrm{K}$ der Kapitalstock. $\mathrm{B}$ ist die Anzahl der ausstehenden Bonds. Wenn unterstellt wird, daß jeder Bond bis in alle Ewigkeit eine Verzinsung von einer Geldeinheit pro Periode abwirft, dann sind die Zinszahlungen mit der Anzahl der Bonds B identisch und der Marktwert der zinspflichtigen Staatsverschuldung beträgt $\mathrm{B} / \mathrm{r}$. Die Zinszahlungen $\mathrm{B}$ sind eine zusätzliche Einkommenskomponente, die der Besteuerung unterworfen werden.

Das Geldmarktgleichgewicht ist:

$$
\begin{aligned}
& \text { (2) } L M: \frac{M}{P}=L(r, Y, M+B / r+K) \text {, } \\
& \text { mit } L_{r}<0 \text { und } L_{w}>0, L_{Y}>0 \text {. }
\end{aligned}
$$

Die staatliche Budgetrestriktion

$$
\text { (3) } \mathrm{G}+\mathrm{B}-\mathrm{T}(\mathrm{Y}+\mathrm{B})=\dot{\mathrm{M}}+\dot{\mathrm{B}} / \mathrm{r}^{462}
$$

besagt, daß die gesamten Staatsausgaben (reale Ausgaben $G$ und die Zinszahlungen auf die Staatsschuld B) durch Steuereinnahmen $T(Y+B)$, Verschuldung über Anleihen $(\dot{B} / r)$ oder Geldschöpfung $(\dot{M})$ finanziert werden müssen. Im langfristigen Gleichgewicht sind $\dot{M}=\dot{B}=0$ und das Budget ist ausgeglichen.

Die Nettoinvestitionen entsprechen der Veränderung des Kapitalstocks:

$$
\text { (4) } I \equiv \dot{K}=I(r, K, Y) \text {. }
$$

Die Investitionstätigkeit stellt einen Anpassungsproze $B$ zum optimalen Kapitalstock dar. Bei $\dot{\mathrm{K}}=0$ ist dieser erreicht und Zins, Einkommen und Kapitalstock befinden sich im Gleichgewicht.

Durch totale Differenzierung von (3) und entsprechende Umformulierung wird der langfristige Staatsausgabenmultiplikator ermittelt:

$$
\text { (5) } \frac{d Y}{d G}=\frac{1+\left(1-T^{\prime}\right) \frac{d B}{d G}}{T^{\prime}} \text {. }
$$

${ }^{462} \dot{\mathrm{X}}=\mathrm{dX} / \mathrm{dt}$. 
dB/dG zeigt die Entwicklung der Bondsausgabe bei einer Erhöhung der Staatsausgaben. Um einen expansiven Ausgabenmultiplikator ( $\mathrm{dY} / \mathrm{dG}>1)$ zu gewährleisten, $\mathrm{mußdB} / \mathrm{dG}>-1$ gelten. ${ }^{463}$

Der Budgetmultiplikator zeigt, wie eine fiskalpolitische Maßnahme langfristig das Einkommen beeinflußt. Vernachläßigt bei dieser Analyse wird jedoch der Anpassungsproze $B$ zum neuen Gleichgewicht. Das komparativ-statische Ergebnis ist nur dann sinnvoll, wenn das System sich tatsächlich zu einem neuen langfristigen Gleichgewicht hin bewegt. Dort gehen keine Vermögenseffekte vom System aus und das Budget ist ausgeglichen.

Dafür lassen s̈ich zwei Stabilitätsbedingungen ableiten. Erstens ist es notwendig, $\mathrm{da} \beta$ die Bondsausgabe expansiv auf das Einkommen wirkt $(\partial \mathrm{Y} / \partial \mathrm{B}>0)$. Zweitens muß dieser Einkommenseffekt ausreichend groß sein, um ein im Zeitverlauf abnehmendes Budgetdefizit zu gewährleisten. Bei zunehmender Bondsausgabe muß der Finanzierungsbedarf des Budgetdefizits sich stetig verringern $(\mathrm{d} \dot{\mathrm{B}} / \mathrm{dB}<0) .464$

In der allgemeinen Form lassen sich die statischen IS und LM Gleichgewichtsbedingungen (1) und (2) umformulieren in

$$
\begin{aligned}
& \text { (6) } \quad \mathrm{Y}=\mathrm{F}(\mathrm{B}, \mathrm{K}, \overline{\mathrm{M}} ; \mathrm{G}) \\
& \text { (7) } \quad \mathrm{r}=\mathrm{H}(\mathrm{B}, \mathrm{K}, \overline{\mathrm{M}} ; \mathrm{G})
\end{aligned}
$$

wobei $\mathrm{M}$ an dieser Stelle konstant gehalten wird.

Durch totale Differenzierung und Umformulierung läßt sich das zwei-GleichungenSystem in Matrixform darstellen und die Jacobi Determinante für die endogenen Variablen $Y$ und $r$ bilden. ${ }^{465}$ Für die exogene Größe $\mathrm{dB} \neq 0$ ergibt sich

$$
\left(\begin{array}{cc}
1-C_{Y}\left(1-T^{\prime}\right)-I_{Y} & -\left(I_{r}-C_{W} B / r^{2}\right) \\
L_{Y} & L_{r}-L_{W} B / r^{2}
\end{array}\right)\left(\begin{array}{c}
d Y \\
d r
\end{array}\right)=\left(\begin{array}{c}
C_{Y}\left(1-T^{\prime}\right)+C_{W} / r \\
-L_{W} / r
\end{array}\right)
$$

Die Jacobi-Determinante ist

\footnotetext{
${ }^{463}$ Bei einem Vergleich zwischen Geld- und Schuldfinanzierung ist die relevante Bedingung $\mathrm{dB} / \mathrm{dG}>0$, was einen größeren Multiplikator bei der Bondfinanzierung bedeutet, siehe Blinder und Solow (1973) S. 326f.

464 Analog für die Kapitalstockentwicklung muß bei zunehmendem Kapitalstock die Investitionstätigkeit mit sinkendem Investitionsbedarf stetig abnehmen $(\mathrm{d} \dot{\mathrm{K}} / \mathrm{dK}<0)$.

${ }^{465}$ Zum Verfahren vergleiche Fußnote 461.
} 


$$
|J| \equiv 1-C_{Y}\left(1-T^{\prime}\right)-I_{Y}+\sigma L_{Y}, \text { mit } \sigma \equiv \frac{I_{r}-\left(B / r^{2}\right) C_{W}}{L_{r}-\left(B / r^{2}\right) L_{W}} .
$$

$|\mathrm{J}|$ gibt die Einkommens- und Zinsveränderungen infolge der expansiven Politik unabhängig von ihrer Finanzierung wieder [d.h. 1/(dY/dG)]. Der Ausdruck setzt sich zusammen aus dem kurzfristigen Multiplikator (einschießlich einem Einkommenseffekt auf die Investitionen) und die Vermögenseffekte der Kapitalgewinne $\left(B / r^{2}\right)$ auf dem Konsum $C_{W}$ und der Liquiditätspräferenz $L_{W}$, die auf den „Crowding-Out" Ausdruck wirken. Hieraus läßt sich eine erste Konsequenz der modifizierten Investitionsfunktion erkennen: Das Vorzeichen des kurzfristigen Multiplikators läßt sich nicht eindeutig determinieren. Bei einer sehr elastischen Investitionsbereitschaft könnte er negativ werden. Von diesem extremen Grenzfall wird jedoch abgesehen. Für die nachfolgende Analyse wird zunächst von einem kurzfristigen expansiven Effekt ausgegangen, das heißt $|\mathrm{J}|>0$.

Mit Hilfe der Cramer'schen Regel läßt sich die Auswirkung der Bondsausgabe auf das Einkommen ermitteln

$$
F_{B} \equiv \frac{\partial Y}{\partial B}=\frac{C_{Y}\left(1-T^{\prime}\right)+\frac{C_{W}-L_{W} \sigma}{r}}{|J|} .
$$

Der erste Ausdruck $\mathrm{C}_{\mathrm{Y}}\left(1-\mathrm{T}^{\prime}\right)$ im Zähler ist der kurzfristige Einkommenseffekt und ist eindeutig positiv. Der zweite Teil erfaßt die Vermögensargumente, deren Auswirkungen einen zentralen Streitpunkt zwischen Kritikern und Verfechtern aktiver Fiskalpolitik darstellt. Der Ausdruck besteht aus den positiven Vermögenseffekt auf Konsum $C_{w}$ und den Vermögenseffekt auf die Geldnachfrage $L_{w}$, der um $\sigma$ - die Zinswirkungen auf Investitionen und auf die Geldnachfrage sowie die vermögensabhängigen Konsum- und Geldnachfrage - gewichtet ist. Die Vermögenszunahme wirkt expansiv auf die Gütermärkte, da annahmegemäß $C_{w}>0$ (Im IS-LM Schema eine Rechtsverschiebung der IS-Ortslinie). Die Geldnachfrage reagiert auch positiv auf den Vermögenszuwachs und verursacht einen Zinsanstieg (eine Linksverschiebung der LM-Ortslinie). Von zentraler Bedeutung für die Beurteilung der Politikmaßnahme ist die Frage, welcher Effekt stärker auftritt. $C_{w}-L_{w} \sigma>0$ ist hinreichend, aber nicht notwendig für $\mathrm{F}_{B}>0$. Ist dagegen $\mathrm{C}_{w}-\mathrm{L}_{\mathrm{w}} \sigma<0$ - mit anderen Worten der Geldnachfrageeffekt überwiegt den Konsumeffekt - dann wird der kurzfristige expansive Impuls durch die später auftretenden negativen Vermögenseffekte gemindert, neutralisiert oder sogar überkompensiert.

Ist der Einkommenseffekt insgesamt positiv $\left(\mathrm{F}_{\mathrm{B}}>0\right)$, aber mit $\mathrm{Cw}-\mathrm{Lw} \sigma<0$, dann läßt sich nicht a priori sagen, ob ein stabiles Ergebnis resultiert (vgl. Abbildung 5), oder der expansive Effekt nicht dafür ausreicht (vgl. Abbildung 4). Dafür müssen 
die dynamische Anpassung des Systems und insbesondere die zweite Stabilitätsbedingung $(\mathrm{d} \dot{\mathrm{B}} / \mathrm{dB}<0)$ untersucht werden.

Mit Hilfe der Gleichungen (6) und (7) werden die staatliche Budgetrestriktion (3) und die Kapitalstockanpassungsgleichung (4) umformuliert:

$$
\begin{aligned}
& \text { (9) } \dot{\mathrm{B}}=\mathrm{f}(\mathrm{B}, \mathrm{K} ; \mathrm{G})=\mathrm{r} \cdot\{\mathrm{G}+\mathrm{B}-\mathrm{T}[\mathrm{F}(\mathrm{B}, \mathrm{K})+\mathrm{B}]\} \\
& \text { (10) } \dot{\mathrm{K}}=\mathrm{g}(\mathrm{B}, \mathrm{K})=\mathrm{I}\{\mathrm{H}(\mathrm{B}, \mathrm{K}), \mathrm{K},[\mathrm{F}(\mathrm{B}, \mathrm{K})+\mathrm{B}]\}
\end{aligned}
$$

Die Eigenschaften des Systems lassen sich in einem zwei Variablen Phasendiagram im B-K Raum darstellen. Zuerst werden die Gleichgewichtspfade $\dot{B}=0$ (und $\dot{\mathrm{K}}=0$ ) in Abhängigkeit von $\mathrm{B}$ und $\mathrm{K}$ ermittelt.

Für die Bondsemission: ${ }^{466}$

$$
\left.\frac{d B}{d K}\right|_{\dot{B}=0}=-\frac{f_{K}}{f_{B}}=-\frac{-r\left(T^{\prime} F_{K}\right)}{r\left(1-T^{\prime}-T^{\prime} F_{B}\right)}
$$

Das Vorzeichen des Ausdrucks bestimmt die Steigung der $\dot{B}=0$ Ortslinie. Sie hängt von der Auswirkung des Kapitalstocks auf das Einkommen $F_{K}$ und dem bereits diskutierten Wert vom $F_{B}$ ab. Die Stabilitätseigenschaften des Systems läßt sich somit nicht zweifellos feststellen. Es lassen sich aber ökonomisch einsichtigen Bedingungen formulieren, unter denen die Stabilität einer schuldfinanzierten Budgetpolitik behauptet werden kann. ${ }^{467}$ Die erste Bedingung lautet, daß infolge einer Kapitalstockerhöhung das Einkommen sinkt:

$$
\text { i) } \quad \mathrm{F}_{\mathrm{K}}<0 \text {. }
$$

Dies scheint plausibel: Ein hoher Kapitalbestand bedeutet eine geringe Differenz zwischen gewünschtem und tatsächlichem Kapitalstock. Durch niedrige Investitionstätigkeit wird das Einkommen negativ beeinflußt. Eine genaue Betrachtung der komparativ-statischen Ableitung ${ }^{468}$ ergibt

\footnotetext{
${ }^{466}$ Mit Hilfe des impliziten Funktionentheorems lassen sich die Eigenschaften der allgemeinen Funktionen untersuchen.
}

${ }^{467}$ Vgl. hierzu Blinder und Solow (1973) S. 334f.

$$
{ }^{468} \mathrm{~F}_{\mathrm{K}}=|\mathrm{J}|^{-1}\left[\begin{array}{cc}
\mathrm{C}_{\mathrm{w}}+\mathrm{I}_{\mathrm{K}} & -\left(\mathrm{I}_{\mathrm{r}}-\mathrm{C}_{\mathrm{w}} \frac{\mathrm{B}}{\mathrm{r}^{2}}\right) \\
-\mathrm{L}_{\mathrm{w}} & \mathrm{L}_{\mathrm{r}}-\mathrm{L}_{\mathrm{w}} \frac{\mathrm{B}}{\mathrm{r}^{2}}
\end{array}\right]=|\mathrm{J}|^{-1}\left(\mathrm{I}_{\mathrm{K}}+\mathrm{C}_{\mathrm{w}}-\mathrm{L}_{\mathrm{w}} \sigma\right)
$$




$$
F_{K}=\frac{I_{K}+C_{w}-L_{w} \sigma}{|J|} .
$$

Überwiegt der negative Effekt der Kapitalstockzunahme auf die Investitionstätigkeit die kumulierten Vermögenseffekte auf dem Konsum und der Geldnachfrage, dann ist $\mathrm{F}_{\mathrm{K}}<0$.

Die oben abgeleitete Stabilitätsbedingung $f_{B} \equiv \partial \dot{B} / \partial B$ ist erfüllt, wenn der expansive Impuls der Bondemission einen vom Grenzsteuersatz bestimmten Schwellenwert überschreiten:

$$
\text { ii) } \quad \mathrm{F}_{\mathrm{B}}>\left(1-\mathrm{T}^{\prime}\right) / \mathrm{T}^{\prime} \text {. }
$$

Infolge einer Bonderhöhung steigen das Einkommen um $F_{B} d B$ und damit auch die Steuereinnahmen um $T^{\prime} F_{B} d B$. Die Kosten betragen dagegen $\left(1-T^{\prime}\right) d B$. Nur wenn die Einnahmen diese Kosten übersteigen, schließt sich die Finanzierunglücke.

Sind die Bedingungen i) und ii) erfüllt, dann ist $-\mathrm{f}_{\mathrm{K}} / \mathrm{f}_{\mathrm{B}}>0$ und somit ist die Steigung $\operatorname{der} \dot{B}=0$ Kurve positiv.

Für die Entwicklung des gleichgewichtigen Kapitalstocks ergibt sich:

$$
\left.\frac{\mathrm{dB}}{\mathrm{dK}}\right|_{\dot{K}^{\prime}=0}=-\frac{\mathrm{g}_{\mathrm{K}}}{\mathrm{g}_{\mathrm{B}}}=-\frac{\mathrm{I}_{\mathrm{K}}+\mathrm{H}_{\mathrm{K}} \mathrm{I}_{\mathrm{r}}+\mathrm{F}_{\mathrm{K}} \mathrm{I}_{\mathrm{Y}}}{\mathrm{I}_{\mathrm{r}} \mathrm{H}_{\mathrm{B}}+\mathrm{I}_{\mathrm{Y}} \mathrm{F}_{\mathrm{B}}} .
$$

Angenommen daß die Bedingung i) $\mathrm{F}_{\mathrm{K}}<0$ erfüllt sei, sind noch zwei unbekannte Größen, $\mathrm{H}_{\mathrm{K}}$ und $\mathrm{H}_{\mathrm{B}}$ unbestimmt.

Die Annahme, daß der Zinssatz aufgrund des Vermögenseffektes des Kapitals steigt, scheint einleuchtend

$$
\text { iii) } \mathrm{H}_{\mathrm{K}}>0 \text {. }
$$

Ist diese Bedingung erfüllt, dann entwickelt sich auch das Kapital entlang eines Anpassungspfad zum Gleichgewicht hin: $\mathrm{g}_{\mathrm{K}} \equiv \mathrm{d} \dot{\mathrm{K}} / \mathrm{dK}<0$.

Schließlich ist die Auswirkung der Bondsausgabe auf die Kapitalstockentwicklung $\left(g_{B}\right)$ zu untersuchen. Die Bondsausgabe erhöht sowohl den Zinssatz $\left(H_{B}>0\right)$ als auch das Einkommen $\left(\mathrm{F}_{\mathrm{B}}>0\right)$. Zu klären gilt, ob die Investitionen durch den höheren Zinssatz stärker abnehmen als sie durch das gestiegene Einkommen zunehmen. Dies hängt von den Elastizitätseigenschaften der Investitionsnachfrage und läßt sich nicht a priori determinieren. Allerdings ist eine explosive Kapitalzunahme als Folge der Bondserhöhung kaum plausibel $\left(g_{B} \equiv d \dot{K} / d B>0\right)$. Unter der Annahme, 
daß bei der Bondsausgabe die Investitionsverdrängung durch den Zinsanstieg dem Einkommensimpuls überwiegt, gilt:

$$
\text { iv) } \mathrm{I}_{\mathrm{r}} \mathrm{H}_{\mathrm{B}}+\mathrm{I}_{\mathrm{Y}} \mathrm{F}_{\mathrm{B}}<0 \text {. }
$$

Gelten iii) und iv), dann ist die $d \dot{K}=0$ Ortslinie negativ geneigt. Die beiden Kurve sind in der Abbildung 31 eingetragen.

\section{Abbildung 31: Dynamische Stabilităt im vermögenserweiteten IS-LM Modell}

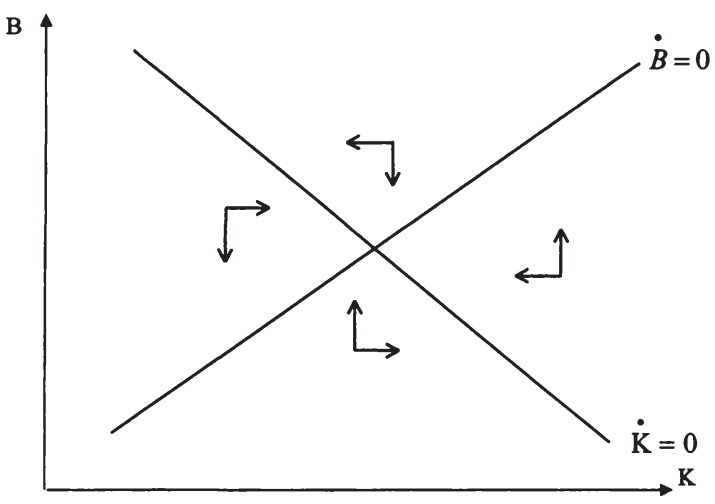

Die Richtung der dynamischen Anpassung des Systems ist durch die Pfeile im Phasendiagram aufgezeigt. $\mathrm{Da} \partial \dot{\mathrm{B}} / \partial \mathrm{B} \equiv \mathrm{F}_{\mathrm{B}}<0$ nimmt mit zunehmender Bondsemission die Emissionsgeschwindigkeit ab. Anders formuliert: Liegt die Anzahl von Bonds B unter ihrem Gleichgewichtswert, wird eine positive Bondsemission den Bondbestand auf sein optimales Niveau hinziehen. Analog gilt für die Investitionstätigkeit, da $g_{K}<0$. Die Investitionen sind positiv, solange der Kapitalstock unter ihrem optimalen Niveau liegt. Sind die Bedingungen i) bis iv) erfüllt, dann ist das System stabil, wie die Abbildung 31 zeigt.

Allerdings sind unter Berücksichtigung der Einkommensabhängigkeit der Investitionen die Stabilitätsbedingungen streng, wie sich unmittelbar an der Bedingung iv) ablesen läßt. $I_{r} H_{B}+I_{Y} F_{B}<0$ erfordert einen relativ geringen Wert für $F_{B}$, insbesondere bei einer starken Akzeleratorwirkung und/oder einer schwachen zinsabhängigen Investitionsnachfrage. Dagegen geht $F_{B}$ auch in die Stabilitätsbedingung ii) $\left(F_{B}>\left(1-T^{\prime}\right) / T^{\prime}\right)$ ein und muß hierfür eine gewisse Mindestgröße erreichen. Ist ii) verletzt, dann ist die Anpassung nur über einen Sattelpfad erreichbar. Das System ist sattelpfadstabil. 
Die Einbeziehung einkommensabhängiger Investitionen hat jedoch auf einem anderen Weg Auswirkungen auf ii). In $F_{B}$ geht der Staatsausgabenmultiplikator dY/dG ein, der wie bereits erwähnt im Falle einer stark einkommensabhängigen Investitionsnachfrage sehr klein oder sogar negativ werden kann. Dadurch ist es auch möglich, da $\beta$ die Bedingung ii) verletzt wird. ${ }^{469}$ Dieses Ergebnis ist unabhängig von den Bedingungen i)-iv) und somit von der Stabilität des Systems.

\section{Zur Berechnung der Entwertung des Schuldenstandes durch die}

\section{Inflation (2. Kapital, Abschnitt II.2)}

Bei einer erwarteten und tatsächlichen Inflation von beispielsweise $10 \%$ ist es klar, da $\beta$ eine nominale Defiziterhöhung von ebenfalls $10 \%$ keinen realen Anstieg bedeutet. In diesem Sinne läßt sich die reale staatliche Budgetrestriktion ermitteln, wenn ihre Komponenten mit einem geeigneten Preisniveau deflationiert werden.

$$
G_{t}+\frac{T r_{t}}{P_{t}}-\frac{T_{t}}{P_{t}}+i_{t-1} \frac{B_{t-1}}{P_{t}}-\frac{A_{t}-A_{t-1}}{P_{t}}=\frac{M_{t}-M_{t-1}}{P_{t}}+\frac{B_{t}-B_{t-1}}{P_{t}}
$$

Bei dieser Deflationierung wurde jedoch vernachläßt, daß der Schuldenbestand in Höhe der Inflation zwischen $\mathrm{t}-1$ bis $\mathrm{t}$ sich entwertet. ${ }^{470}$ Die oben beschriebene Anpassung des Nominalzinses infolge des inflationsbedingten Einkommensanstiegs zieht einen Wertminderungseffekt mit sich und bedeutet, daß selbst bei vollkommen antizipierter Inflation eine Schuldentilgung stattfindet.

Dieser Effekt läßt sich erfassen, wenn die nominale Verzinsung des Schuldenbestandes explizit als Zusammensetzung des realen Zinssatzes und der erwarteten Inflationsrate in die staatliche Budgetbeschränkung eingesetzt wird. Der Realzins wird von Preisänderungen nicht tangiert und sei konstant. ${ }^{471}$ Es herrsche vollkommene Voraussicht über die zukünftige Preisentwicklung, so daß die in t-1 gebildete Inflationserwartung der tatsächlichen Inflationsrate $\pi_{\mathrm{t}}$ entspricht. ${ }^{472}$ Unter Berück-

\footnotetext{
${ }^{469}$ Wird der kurzfristige Multiplikator negativ, dann werden die Bedingung i), iii) und iv) auch verletzt. Dann wird je nach relativer Steigung der Kurven entweder das System explodieren oder sich ein Gleichgewicht nur über einen Sattelpfad einstellen.

${ }^{470}$ Ausführlich hierzu siehe z. B. Siegel (1979) S. 84f., Huber (1990) S.20ff., Miller (1982) S. 50ff.

${ }^{471}$ Von anderen Einflüssen auf den Realzins, wie z. B. variablen Risikoprämien oder Transaktionskosten, wird damit abstrahiert.

472 Diese Vereinfachung hat keine wesentlichen Konsequenzen für die Analyse, da es sich hier um eine Bewertungsdivergenz handelt, die unabhängig von den Erwartungen der Inflation aguftritt.
} 
sichtigung dieser temporären Komponente läßt sich die Fisher Gleichung (bei vollkommener Voraussicht, d. h. $\pi^{e}=\pi$ ) schreiben:

$$
\text { (3) } i_{t-1}=\bar{r}+\pi_{t} \text {. }
$$

Durch entsprechende Einsetzung der Fisher Gleichung in (2) und einige Umformungen und Vereinfachungen ${ }^{473}$ läßt sich die reale Budgetbeschränkung umformen in

(4) $G_{t}+\frac{T r_{t}}{P_{t}}-\frac{T_{t}}{P_{t}}+\bar{r} \frac{B_{t-1}}{P_{t}}-\frac{A_{t}-A_{t-1}}{P_{t}}=\frac{M_{t}-M_{t-1}}{P_{t}}+\frac{B_{t}-B_{t-1}}{P_{t}}-\pi t \frac{B_{t-1}}{P_{t}}$.

Die Differenz zwischen (2) und (4) beträgt

$$
\begin{aligned}
& {\left[\frac{M_{t}-M_{t-1}}{P_{t}}+\frac{B_{t}-B_{t-1}}{P_{t}}\right]-\left[\frac{M_{t}-M_{t-1}}{P_{t}}+\frac{B_{t}-B_{t-1}}{P_{t}}-\pi_{t} \frac{B_{t-1}}{P_{t}}\right]} \\
& =i_{t-1} \frac{B_{t-1}}{P_{t}}-\bar{r} \frac{B_{t-1}}{P_{t}} \text { oder: } \pi_{t} \frac{B_{t-1}}{P_{t}}
\end{aligned}
$$

Dieser Unterschied gibt die implizite Tilgung des Schuldenstandes $B_{t-1} / P_{t}$ infolge der Inflation zwischen $t$ und t-1 an. Anders formuliert entspricht er der realen Budgetentlastung für den Staat durch die Berechnung der nominalen Zinszahlungen auf der Staatsschuld in der Budgetbeschränkung statt der realen Zinszahlungen, die ihn tatsächlich belasten.

\section{Berechnung der Veränderung der Schuldenquote, aufgeteilt nach Inflations-, Wachstums- und Neuverschuldungseffekten} (4. Kapitel, Abschnitt I.2)

Ausgehend von einem Basisjahr 1978 wurden die jährlichen Auswirkungen des nominellen Wachstums auf den Nenner und des Finanzierungsdefizits auf den Zähler der Bruttoverschuldungsquote in den darauffolgenden Jahren berechnet. Die Abbildung 32 zeigt, wie die so berechnete Bruttoverschuldungsquote annähernd der von der Bank of England ermittelten Verschuldungsquote entspricht.

473 Geldbestände $\left(\mathrm{M}_{\mathrm{t}-1}\right)$ werden nicht nominal verzinst. Daraus ergibt sich eine negative reale Verzinsung auf Geld in Höhe der Inflationsrate, die sich bei einer Betrachtung der Finanzierung der Neuverschuldung positiv auswirkt. Bei dieser Herleitung, die an Änderungen des Schuldenbe-

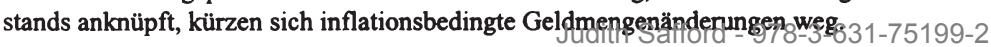




\section{Abbildung 32: Vergleich zwischen berechneter und tatsächlicher Verschuldungsquote}

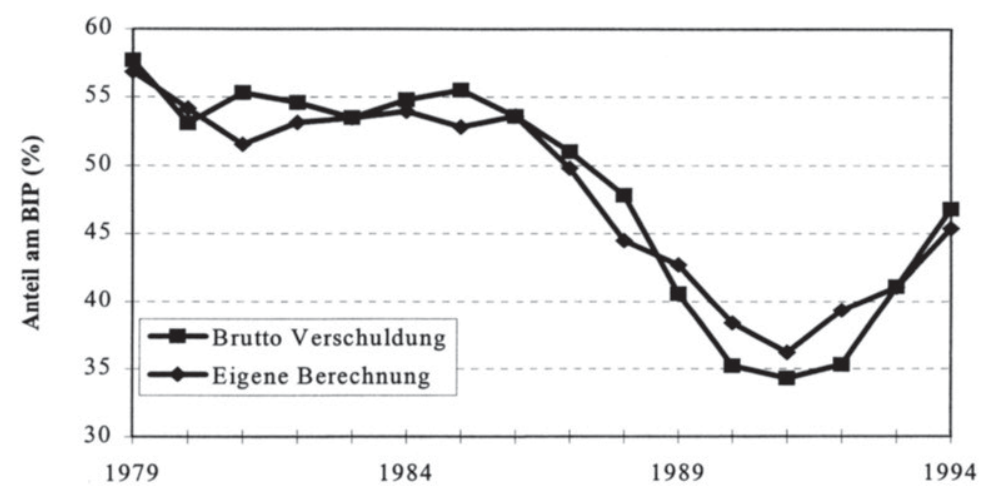

Quelle: CSO, BoEQB und eigene Berechnungen

In einem zweiten Schritt wurde das nominelle Wachstum in seine beiden Bestandteile Inflation und reales Wachstum aufgespalten. Die verwendeten Zeitreihen sind in der Tabelle „Eine Zerlegung der Veränderung der Schuldenquote“ im Anhang dargestellt.

\section{Methodische Anmerkungen zur Berechnung der Inflationsbereini- gung des Finanzierungsdefizits (4. Kapitel, Abschnitt I.3)}

Bei der Berechnung der Inflationsbereinigung $\pi_{t}\left(B_{t-1}\right)$, wie sie im Abschnitt I.3 abgeleitet wurde, gibt es verschiedene methodische Schwierigkeiten bei der Definition und Bewertung der gewählten Variablen: ${ }^{474}$

i) Bei der Definition der Nettoschuld $B_{t-1}$ kann zwischen jährlichen Bestandsgrößen und kumulierten Stromgrößen, berechnet von einem Ausgangsbestand, gewählt werden. Wie bereits erwähnt, sind diese Zeitreihen aus verschiedenen Gründen nicht miteinander konsistent (vgl. Fußnote 330). In dieser Abhandlung wurden die Netto-Schuldbestände nach der BoE-Statistik gewählt. Weil bei den indexierten 
Schuldverschreibungen Wertveränderungen infolge Inflation definitionsgemäß ausgeschlossen sind, wurden diese von der Staatsschuld $\left(B_{t-1}\right)$ abgezogen.

ii) Die ermittelten Bestände können nach dem Marktwert- oder dem Nominalwertprinzip bewertet werden. Für die meisten finanziellen Verbindlichkeiten und Forderungen ist dies unwichtig, aber gerade bei der Staatsschuld können Zinsschwankungen zu Bewertungsunterschieden zwischen Markt- und Nominalwert von bis zu $10 \%$ führen. Eine Bewertung nach dem Marktwertprinzip ist schwierig, weil die Kursschwankungen nicht nur auf Änderungen der Inflationsrate, sondern auch des Realzins zurückzuführen sind. Es ist jedoch kaum möglich, diese zwei Einflüsse voneinander zu isolieren, was die Berechnung nach dem Marktwertprinzip vor groBe praktische Probleme stellt. ${ }^{475}$ Die gewählte BoE-Statistik bewertet die Staatsschuld nach dem Nominalwertprinzip. ${ }^{476}$

iii) Für die Deflationierung kann entweder ein ex-ante oder ex-post Verfahren gewählt werden. Für das ex-post Verfahren wird die aktuelle Inflationsrate verwendet. Das ex-ante Verfahren berücksichtigt die erwartete Inflationsrate. Diese wird ermittelt als Unterschied zwischen realem und nominalem Ertrag auf die jeweils indexierte und nicht indexierte Staatsschuld mit gleich langen Laufzeiten. Die ermittelte Inflationsrate ist folglich ein gewichteter Durchschnitt der Inflationserwartungen bis zur Fälligkeit des Schuldpapiers. Deswegen führt die Anwendung der ex-ante Messung der Inflation dazu, daß die Wertminderung des Finanzvermögens im Zeitverlauf relativ gleichmäßig verläuft. Dagegen schwanken mit der expost Methode die Wertänderungen der Schuld entsprechend der aktuellen Inflationsrate. Zudem ist anzumerken, daß die ex-post Methode eine eventuelle Anpassung des Nominalzins durch die Inflationserwartungen nicht berücksichtigt und somit die Umverteilung zugunsten des Staates möglicherweise überschätzt. Wegen der großen methodischen Schwierigkeiten bei der Schätzung der Inflationserwartungen wurde jedoch die ex-post Methode gewählt. ${ }^{477}$

iv) Schließlich muß zwischen verschiedenen Preisindices gewählt werden. Dies ist vor allem wichtig, wenn ein signifikanter Anteil der betroffenen Vermögensteile in

${ }^{475}$ Die Bestandsgröße in der Nationalstatistik berücksichtigt Wechselkursveränderungen nicht, welche zu Wertveränderungen führen. Dies ist allerdings für die staatlichen Verbindlichkeiten weniger wichtig, weil sie im wesentlichen auf Pfund-Sterling (circa 95\%) lauten.

476 Dieses Vorgehen wird auch bei der Bank of England-Statistik gewählt, siehe hierzu, Taylor and Threadgold (1979), Kennedy (1989) und Peters (1990). Cukierman et al. (1985) wählen das Marktwertprinzip.

${ }^{477}$ Miller (1982) und Cukierman et al. (1985) wählen die ex-ante Methode. Millers (1982) Ergebnisse sind der ex-post Methode von Taylor and Threadgold (1979) ähnlich. Nach den Schätzungen von Cukierman et al. (1985) machte für das Vereinigte Königreich zwischen 1975 und 1982 die gesamte Umverteilung des Vermögens an den Staat jährlich 1,61\% vom BIP aus. Davon waren nur $0,1 \%$ auf die unerwartete Umverteilung zurückzuführen. 
Fremdwährungen gehalten werden, was jedoch bei der britischen Staatsschuld nicht der Fall ist. Für Studien, welche die Vermögensverluste der Haushalte in den Vordergrund stellen, ${ }^{478}$ erscheint der Konsumentenpreisindex angebracht, weil das Endziel der wirtschaftlichen Tätigkeit annahmegemäß der Konsum ist. Für diese Abhandlung erschien der Deflator des Bruttoinlandsproduktes angemessen, um die Konsistenz mit den anderen Zeitreihen zu bewahren. Die zwei Inflationsraten verliefen ohnehin relativ ähnlich.

Um zum inflationsbereinigten Finanzierungsdefizit zu gelangen, wurde dann die ermittelte Inflationsbereinigung vom konventionellen Finanzierungsdefizit abgezogen. 


\section{Wichtige Privatisierungen und ihre Erlöse}

\begin{tabular}{|c|c|c|c|}
\hline Unternehmen & Industriezweig & Verkaufsdatum & $\begin{array}{l}\text { Brutto Erlös } \\
\text { (Mill. £) }\end{array}$ \\
\hline British Petroleum & Erdöl & $1979-83$ & 827 \\
\hline British Technology Group (NEB) & Technologie & $1979-1984$ & 190 \\
\hline British Aerospace & Luft- und Raumfahrt & $1981-85$ & 389 \\
\hline British Sugar Corporation & Zucker-Raffinerie & Juli 1981 & 44 \\
\hline Cable \& Wireless & Telekommunikation & $1981-85$ & 1.045 \\
\hline British Steel Corporation & Steel & 1981 & 592 \\
\hline Amersham & Radioakt. Chemikalien & Feb 82 & 64 \\
\hline National Freight Corporation & Transport & 1982 & 7 \\
\hline Britoil & Erdöl & $1982-85$ & 1.052 \\
\hline Assoc. British Ports & Seehäfen & $1983-84$ & 97 \\
\hline Internat. Aeradio & Luftfahrt-Kommunikation & März 83 & 60 \\
\hline British Rail Hotels & Hotels & März 83 & 51 \\
\hline United Medical Ent. & Medizinische Geräte & 1983 & 17 \\
\hline Wytch Farm & Erdöl & Mai 84 & 82 \\
\hline Enterprise Oil & Erdöl & Juni 84 & 380 \\
\hline Sealink & Häfen/Fähren & Juli 84 & 66 \\
\hline Jaguar & Autos & Juli 84 & 297 \\
\hline British Telecom & Telekommunikation & $1984-94$ & 11.200 \\
\hline National Bus Company & Transport & $1986-88$ & 325 \\
\hline Royal Ordonance & Rüstung & $1986-89$ & 236 \\
\hline National Seed Dev. Org. & Landwirtschaft & 1987 & 66 \\
\hline Rover Group (BL) & Autos & $1987-88$ & 217 \\
\hline Giroleasing & & 1990 & 342 \\
\hline British Gas & Gas & April 92 & 600 \\
\hline Scottish Electricity & Strom & Mai 92 & 800 \\
\hline Electricty & Strom & Sept 92 & 1.400 \\
\hline $\begin{array}{l}\text { Electricity (England and Scot- } \\
\text { land) }\end{array}$ & Strom & April 93 & 1.400 \\
\hline N. Ireland Electricty Service & Strom & $1993-94$ & 400 \\
\hline Electricity Debenture & Strom & April 94 & 400 \\
\hline British Coal & Kohlenindustrie & Dez. 94 & 700 \\
\hline \multirow{2}{*}{\multicolumn{3}{|c|}{ Andere }} & 716 \\
\hline & & cs & \\
\hline
\end{tabular}




\section{Statistische Grundlage (3. und 4. Kapitel)}

\begin{tabular}{|c|c|c|c|c|c|}
\hline \multicolumn{6}{|c|}{ Die Bruttoverschuldung seit 1975} \\
\hline \multicolumn{6}{|c|}{ Anteil am BIP / BSP } \\
\hline Jahr & $\begin{array}{l}\text { Konsolidierter } \\
\text { Schuldenstand }\end{array}$ & BIP & $\begin{array}{l}\text { Konsolidierte } \\
\text { Schuldenquote }\end{array}$ & $\begin{array}{c}\text { Brutto- } \\
\text { Schuldenquote }\end{array}$ & $\begin{array}{c}\text { Brutto- } \\
\text { Schuldenquote }\end{array}$ \\
\hline 1975 & $46^{\prime} 856$ & $105^{\prime} 400$ & 44.46 & 66.3 & \\
\hline 1976 & $57^{\prime} 162$ & $124^{\prime} 600$ & 45.88 & 66.3 & \\
\hline 1977 & $67 ' 325$ & $145^{\prime} 400$ & 46.30 & 66.2 & \\
\hline 1978 & $77^{\prime} 841$ & $167^{\prime} 814$ & 46.39 & 61.9 & 58.6 \\
\hline 1979 & $86^{\prime} 087$ & $197^{\prime} 831$ & 43.52 & 57.7 & 54.9 \\
\hline 1980 & $94^{\prime} 830$ & $231^{\prime} 272$ & 41.00 & 53.1 & 54.1 \\
\hline 1981 & $112^{\prime} 483$ & $254^{\prime} 294$ & 44.23 & 55.3 & 54.3 \\
\hline 1982 & $122^{\prime} 065$ & $278^{\prime} 887$ & 43.77 & 54.6 & 52.9 \\
\hline 1983 & $133^{\prime} 563$ & $304^{\prime} 314$ & 43.89 & 53.5 & 52.9 \\
\hline 1984 & $151^{\prime} 191$ & $325^{\prime} 091$ & 46.51 & 54.8 & 54.4 \\
\hline 1985 & $167 ' 528$ & $356^{\prime} 083$ & 47.05 & 55.5 & 52.7 \\
\hline 1986 & $180^{\prime} 318$ & $382^{\prime} 942$ & 47.09 & 53.6 & 51.1 \\
\hline 1987 & $191 ' 598$ & $421 ' 198$ & 45.49 & 51 & 48.6 \\
\hline 1988 & $202^{\prime} 757$ & $467^{\prime} 863$ & 43.34 & 47.8 & 42.2 \\
\hline 1989 & $192 ' 349$ & $512^{\prime} 221$ & 37.55 & 40.6 & 36.7 \\
\hline 1990 & $185 ' 477$ & $550^{\prime} 198$ & 33.71 & 35.2 & 34.6 \\
\hline 1991 & $189 ' 291$ & $575^{\prime} 321$ & 32.90 & 34.3 & 35.3 \\
\hline 1992 & $200^{\prime} 450$ & $597 ' 242$ & 33.56 & 35.3 & 41.2 \\
\hline 1993 & $245^{\prime} 093$ & $630^{\prime} 707$ & 38.86 & 41.1 & 47.1 \\
\hline 1994 & & $668^{\prime} 866$ & & 46.8 & 54.5 \\
\hline Quelle & Barro/Grilli 1994 & $\mathrm{CSO}, \mathrm{CAOB}$ & & BoE & OECD \\
\hline
\end{tabular}




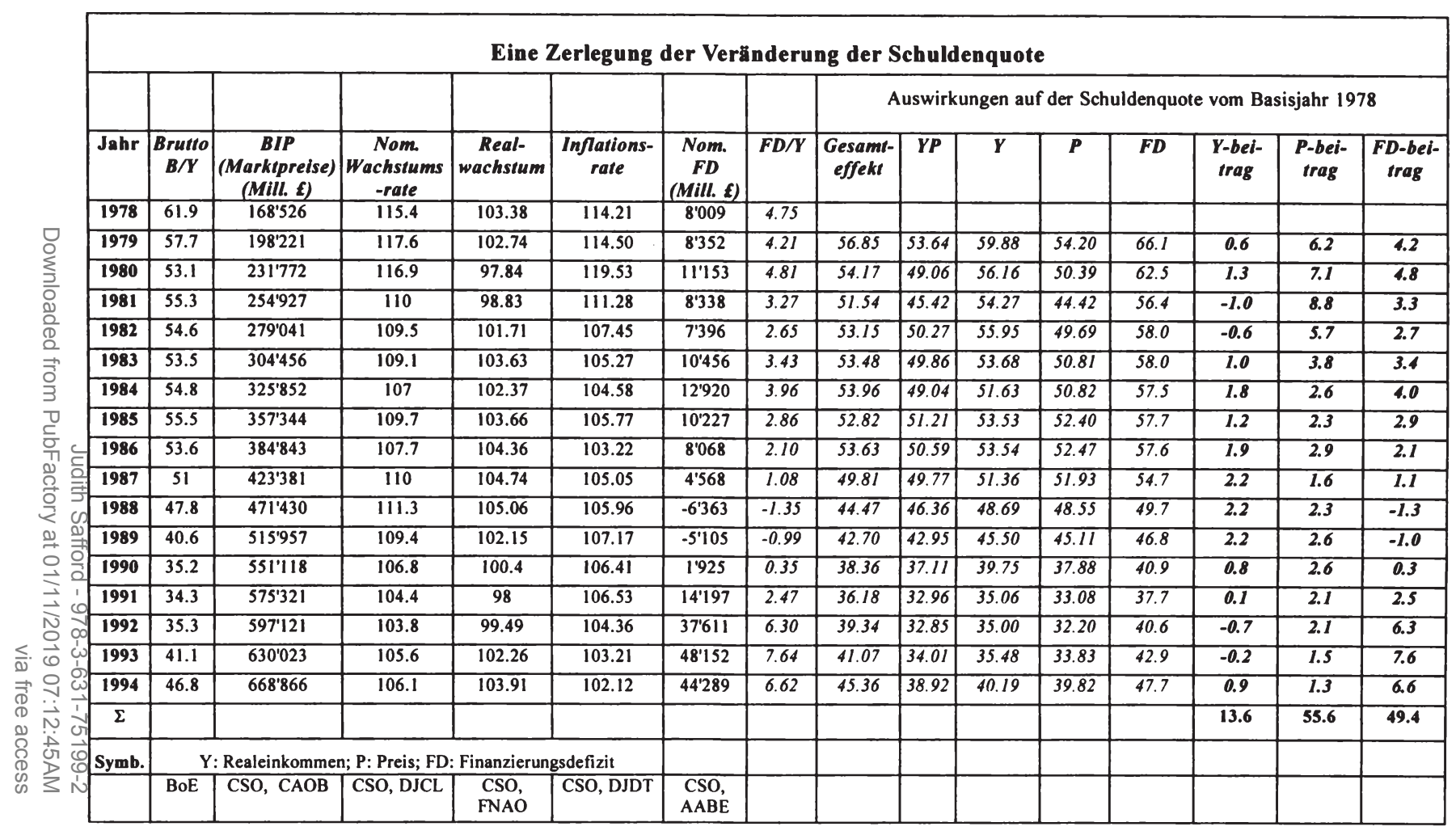




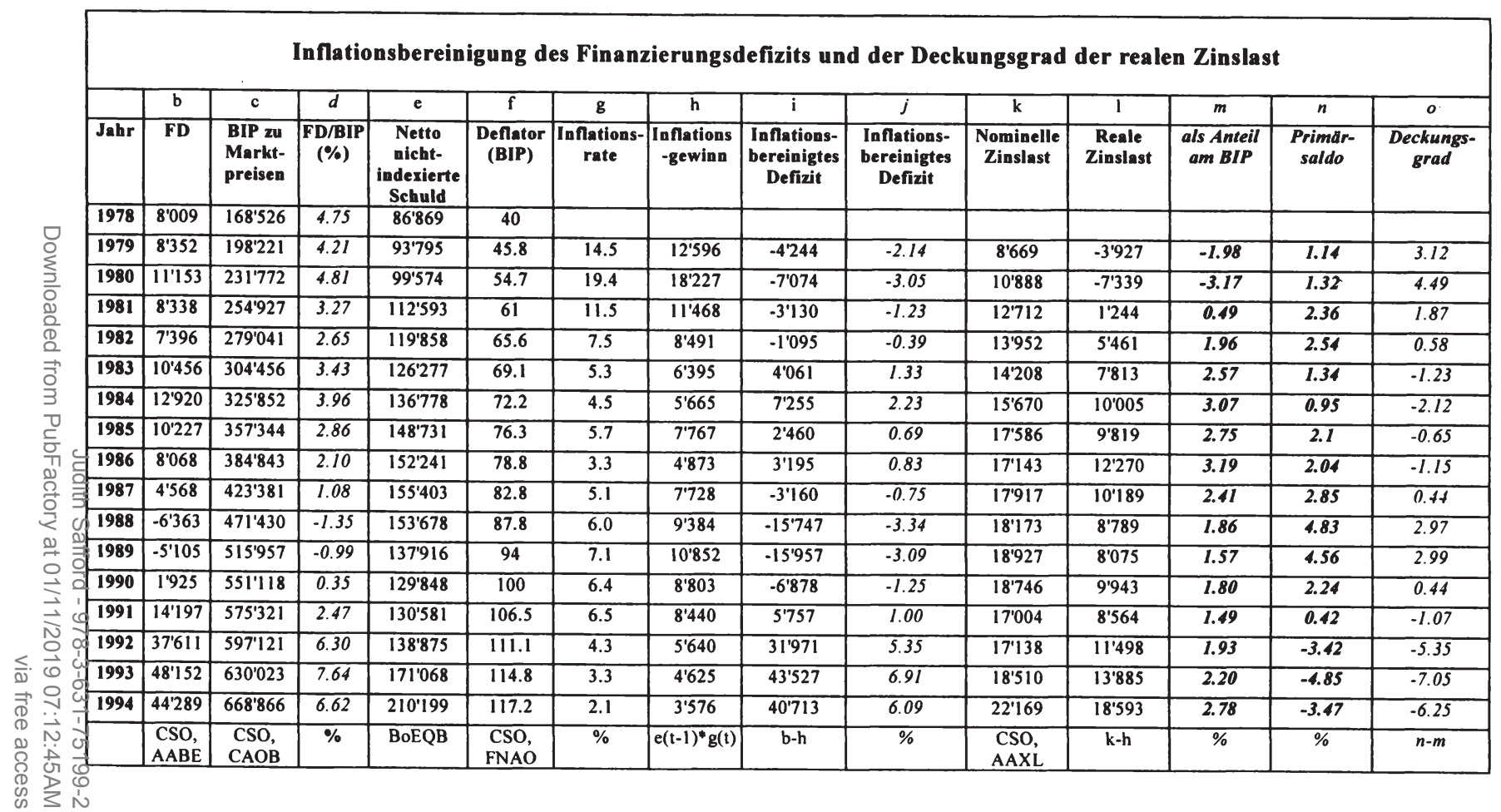




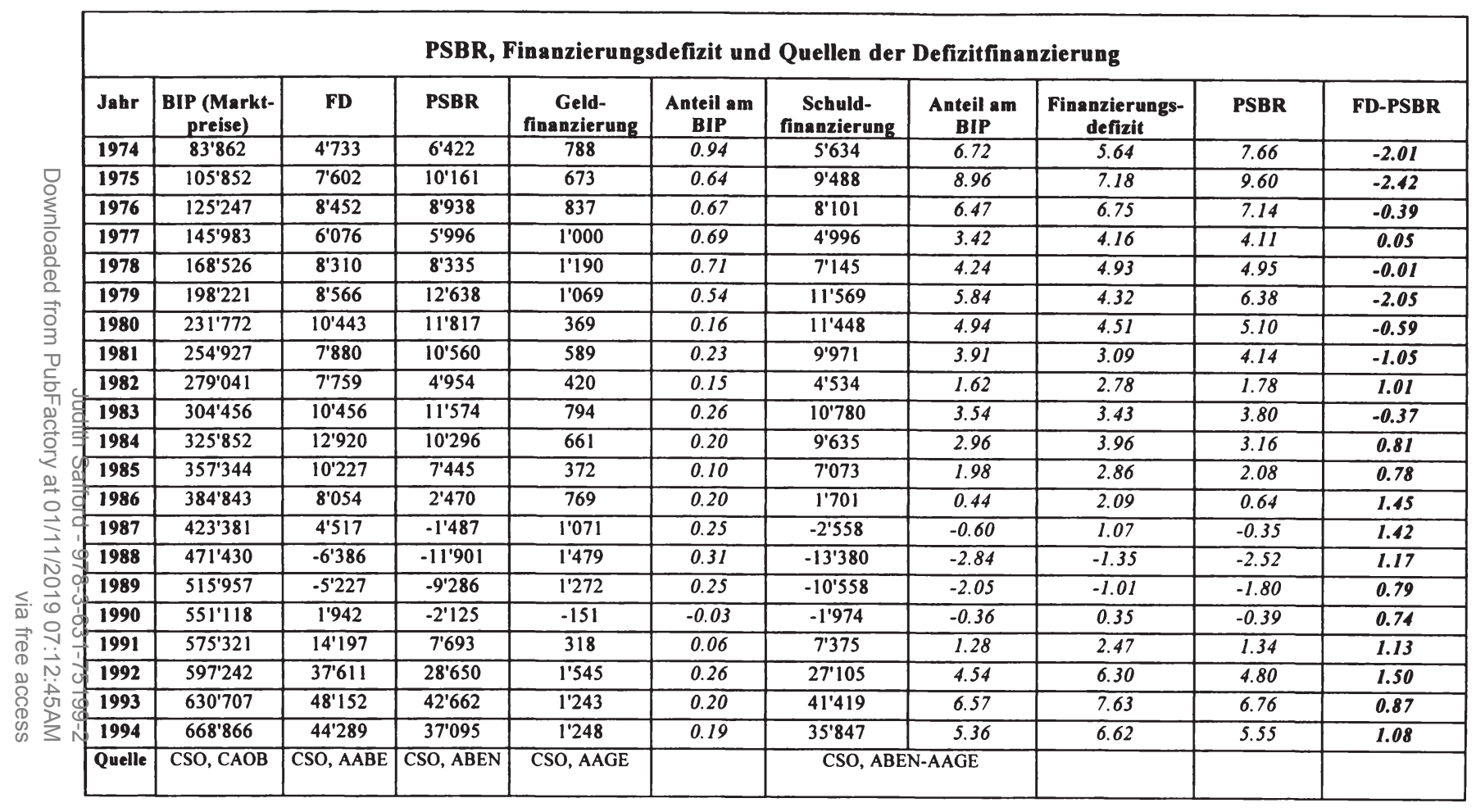


Die Finanzierungsdefizite der öffentlichen Institutionen

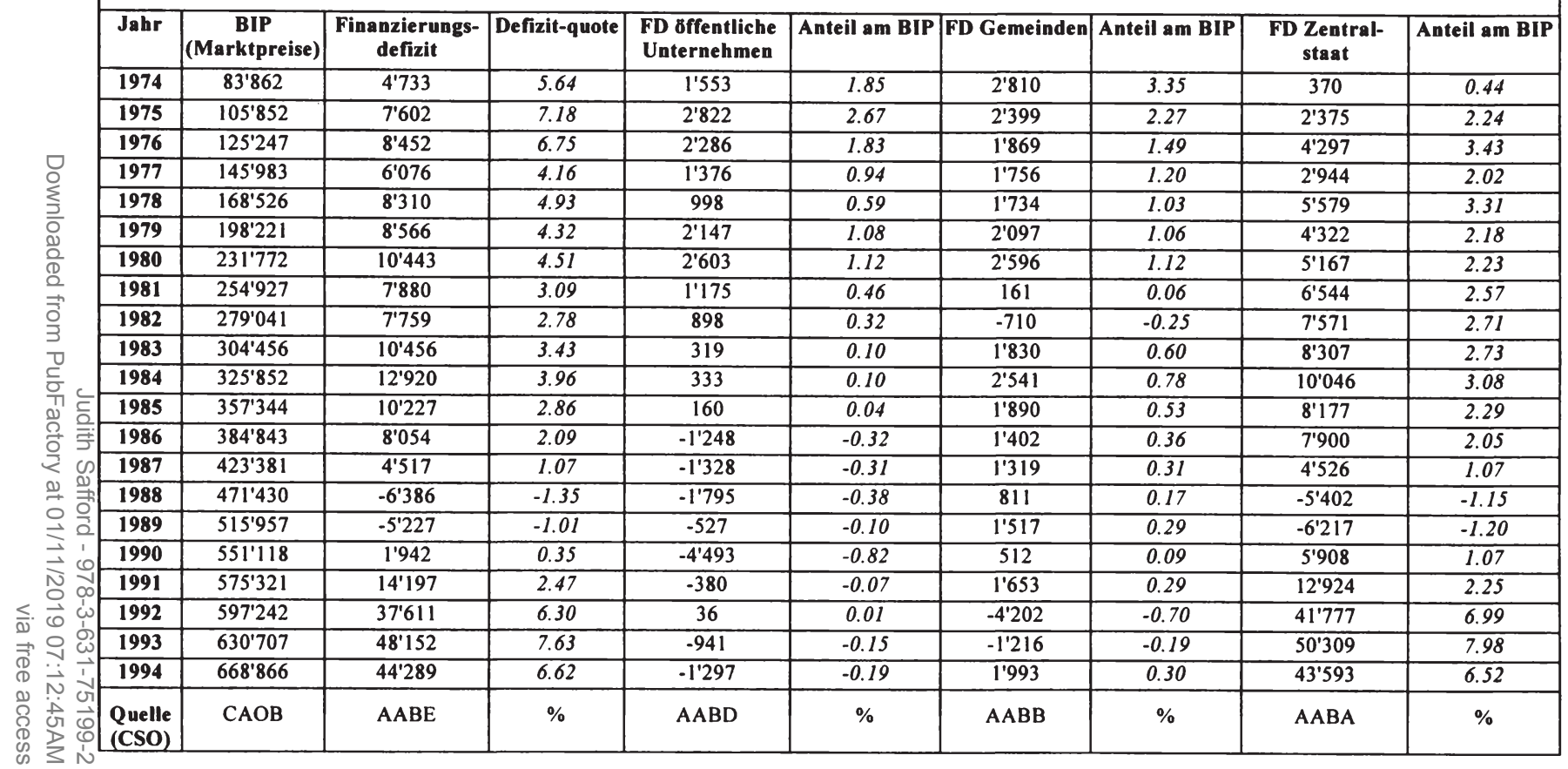




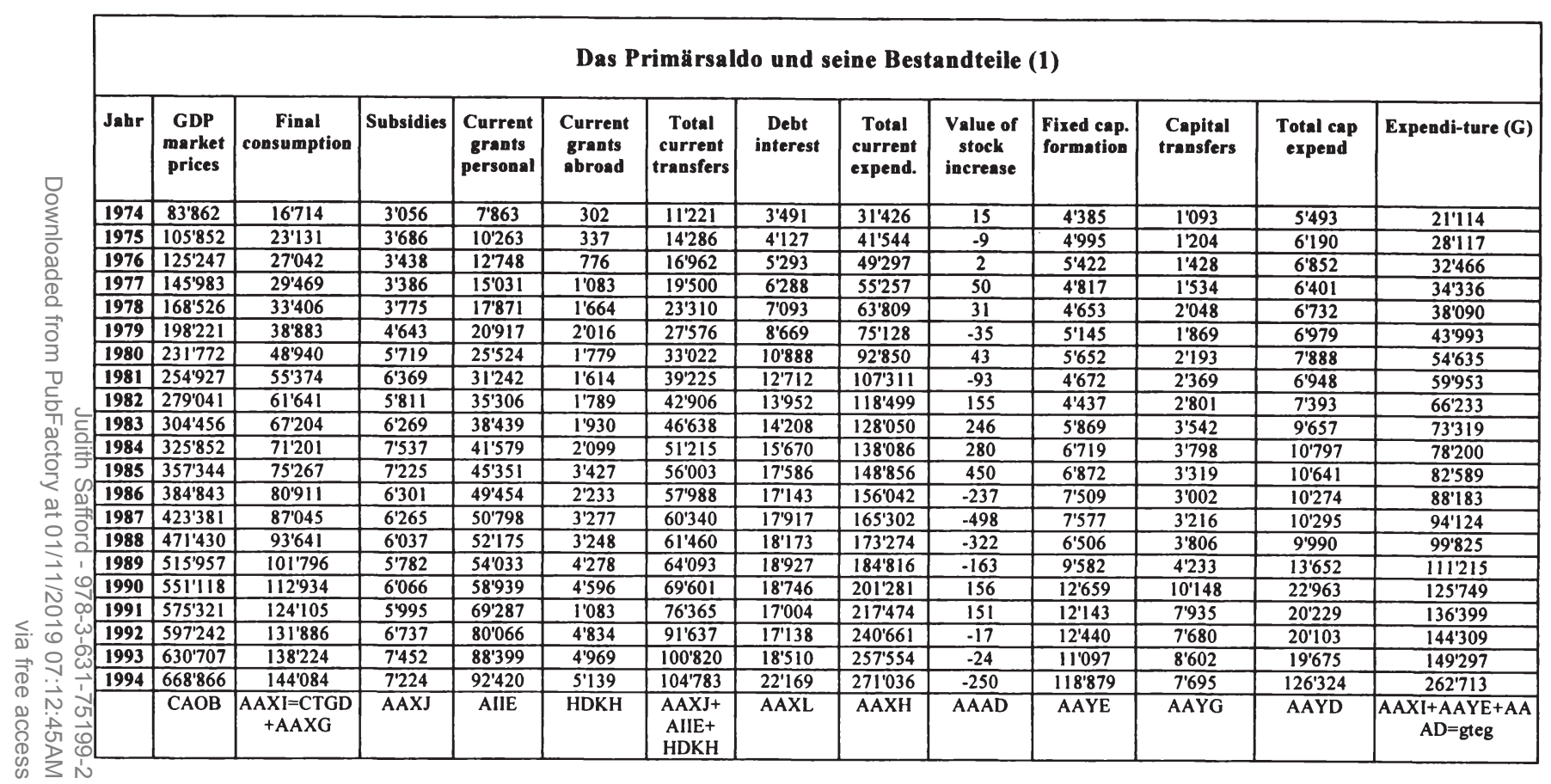


Das Primärsaldo und seine Bestandteile (2)

\begin{tabular}{|c|c|c|c|c|c|c|c|c|c|c|c|c|c|c|}
\hline & $\begin{array}{c}\text { Total } \\
\text { transfers } \\
\text { (Tr) }\end{array}$ & $\begin{array}{c}\text { Total } \\
\text { expendi- } \\
\text { ture (1) }\end{array}$ & \begin{tabular}{|c|} 
Taxes, Soc. \\
Securitiy, \\
com. \\
Charge
\end{tabular} & $\begin{array}{c}\text { Cap. } \\
\text { taxes and } \\
\text { receipts }\end{array}$ & $\begin{array}{l}\text { Zinsen, } \\
\text { Divi- } \\
\text { denden } \\
\text { u. a. }\end{array}$ & \begin{tabular}{|c|} 
Total \\
revenue (1) \\
(T)
\end{tabular} & $\begin{array}{c}\text { Primär- } \\
\text { saldo }\end{array}$ & $\begin{array}{c}\text { Steuern und } \\
\text { Beteili- } \\
\text { gungen }\end{array}$ & \begin{tabular}{|c|} 
Oil \\
revenus
\end{tabular} & Ausgaben & $\begin{array}{c}\text { Ein- } \\
\text { nahmen }\end{array}$ & $\begin{array}{c}\text { Primilr- } \\
\text { aberschua }\end{array}$ & Konsum & Reale Ausgaben \\
\hline \multirow{8}{*}{ 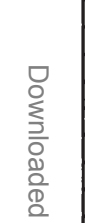 } & 12314 & $36^{\prime} 919$ & $32 ' 861$ & 878 & 31771 & $33 ' 739$ & -311 & $29^{\prime} 968$ & & 39.86 & 40.23 & 0.37 & 19.93 & 25.18 \\
\hline & 15490 & $47 \cdot 734$ & $42 ' 157$ & 797 & 4496 & $42^{\prime} 954$ & 653 & 38.458 & & 41.20 & 40.58 & -0.62 & 21.85 & 26.56 \\
\hline & $18^{\prime} 390$ & 56.149 & $49^{\prime} 170$ & 813 & 5425 & $49^{\prime} 983$ & 873 & 44,558 & & 40.60 & 39.91 & -0.70 & 21.59 & 25.92 \\
\hline & $21^{\prime} 034$ & $61^{\prime} 658$ & $56^{\prime} 134$ & 824 & $6 ' 102$ & $56^{\prime} 958$ & $-1 ' 588$ & 50.856 & 200 & 37.93 & 39.02 & 1.09 & 20.19 & 23.52 \\
\hline & $25{ }^{\prime} 358$ & $70 ' 541$ & $62 ' 395$ & 834 & $6^{\prime} 666$ & $63^{\prime} 229$ & 219 & 56.563 & 600 & 37.65 & 37.52 & -0.13 & 19.82 & 22.60 \\
\hline & $29 ' 445$ & $82^{\prime} 107$ & $74 ' 673$ & 11015 & $7 ' 687$ & $75^{\prime} 688$ & $.2 ' 250$ & $68^{\prime} 001$ & $2 \cdot 300$ & 37.05 & 38.18 & 1.14 & 19.62 & 22.19 \\
\hline & $35 ' 215$ & $100^{\prime} 738$ & $91 ' 718$ & $1 ' 181$ & $9 ' 499$ & $92 ' 899$ & -31049 & 83.400 & $3 ' 700$ & 38.77 & 40.08 & 1.32 & 21.12 & 23.57 \\
\hline & $41 ' 594$ & 114259 & $106^{\prime} 047$ & $1 ' 507$ & $10^{\prime} 220$ & $107^{\prime} 554$ & $-6^{\prime} 007$ & $97 ' 334$ & $6 ' 500$ & 39.83 & 42.19 & 2.36 & 21.72 & 23.52 \\
\hline \multirow{2}{*}{$\frac{\overrightarrow{3}}{3}$} & $45^{\prime} 707$ & 125892 & 117413 & $1 ' 618$ & $11^{\prime} 954$ & $119^{\prime} 031$ & $-7^{\prime} 091$ & 107077 & $7 ' 800$ & 40.12 & 42.66 & 2.54 & 22.09 & 23.74 \\
\hline & $50 ' 180$ & 137707 & $125 ' 910$ & 1660 & $10^{\prime} 493$ & $127 ' 570$ & $-4^{\prime} 071$ & $117^{\prime 077}$ & $8^{\prime} 800$ & 40.56 & 41.90 & 1.34 & 22.07 & 24.08 \\
\hline \multirow{3}{*}{$\begin{array}{l}0 \\
0 \\
\frac{c}{0} \\
\frac{1}{T}\end{array}$} & $55^{\prime} 013$ & $148^{\prime} 883$ & $134{ }^{\prime} 464$ & 1,832 & $10^{\prime} 364$ & 136296 & .3083 & $125^{\prime} 932$ & $12^{\prime} 000$ & 40.88 & 41.83 & 0.95 & 21.85 & 24.00 \\
\hline & $59^{\prime} 322$ & $159 ' 497$ & 147.059 & $2 ' 371$ & $12^{\prime} 418$ & $149^{\prime} 430$ & $-7 ' 519$ & 137012 & 11500 & 39.71 & 41.82 & 2.10 & 21.06 & 23.11 \\
\hline & $60^{\prime} 990$ & $166^{\prime} 316$ & $153 ' 833$ & $3^{\prime} 181$ & $12^{\prime} 103$ & $157^{\prime} 014$ & $-7 ' 841$ & $144^{\prime} 911$ & $6^{\prime} 100$ & 38.76 & 40.80 & 2.04 & 21.02 & 22.91 \\
\hline \multirow{2}{*}{ 光 함 } & $63 ' 556$ & 175.597 & $166^{\prime} 466$ & 3286 & $12^{\prime} 276$ & $169^{\prime} 752$ & $-12^{\prime} 072$ & $157^{\prime} 476$ & $5 ' 200$ & 37.24 & 40.09 & 2.85 & 20.56 & 22.23 \\
\hline & $65^{\prime} 266$ & $183^{\prime} 264$ & $183^{\prime} 471$ & $4^{\prime} 384$ & $13^{\prime} 037$ & $187^{\prime} 855$ & $-22^{\prime} 764$ & $174 ' 818$ & $5^{\prime} 000$ & 35.02 & 39.85 & 4.83 & 19.86 & 21.17 \\
\hline \multirow{2}{*}{$\begin{array}{ll}2 & \text { रे } \\
0 & 0\end{array}$} & $68^{\prime} 326$ & $198^{\prime} 468$ & $198^{\prime} 339$ & $4 ' 707$ & $14^{\prime} 616$ & $203^{\prime} 046$ & $-23^{\prime} 505$ & $188^{\prime} 430$ & $4 ' 200$ & 34.80 & 39.35 & 4.56 & 19.73 & 21.56 \\
\hline & $79^{\prime} 749$ & $224 ' 244$ & $213^{\prime} 386$ & $4^{\prime} 440$ & $14 ' 537$ & 2178826 & $-12^{\prime} 328$ & $203 ' 289$ & $4^{\prime} 000$ & 37.29 & 39.52 & 2.24 & 20.49 & 22.82 \\
\hline \multirow{4}{*}{ 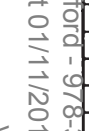 } & $84^{\prime} 300$ & $237^{\prime} 703$ & $219^{\prime} 572$ & 3'558 & $14^{\prime} 141$ & $223 \prime 130$ & -21431 & $208^{\prime} 989$ & $3^{\prime} 000$ & 38.36 & 38.78 & 0.42 & 21.57 & 23.71 \\
\hline & 99'317 & $260^{\prime} 764$ & $220^{\prime} 401$ & 2829 & $13^{\prime} 782$ & $223 ' 230$ & 20,396 & $209^{\prime} 448$ & $22^{\prime} 000$ & 40.79 & 37.38 & -3.42 & 22.08 & 24.16 \\
\hline & $109^{\prime} 422$ & $277 ' 229$ & $225 ' 767$ & $2 ' 500$ & $13 ' 532$ & 228267 & $30^{\prime} 452$ & $214^{\prime} 735$ & $1 ' 600$ & 41.02 & 36.19 & -4.83 & 21.92 & 23.67 \\
\hline & $112^{\prime} 478$ & $397 ' 360$ & $242 ' 723$ & $2 ' 797$ & $14^{\prime} 032$ & $245 ' 520$ & $129 ' 671$ & $231^{\prime} 488$ & $2 ' 200$ & 60.82 & 36.71 & -24.11 & 21.54 & 39.28 \\
\hline 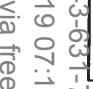 & $\begin{array}{l}\text { AAXJ+AI } \\
\text { IE+HDK } \\
\text { H+AAYG }\end{array}$ & $\begin{array}{r}\text { AAYD+ } \\
\text { AAXH }\end{array}$ & AAXA & GTDZ & $\begin{array}{c}\mathrm{AAXA}+\mathrm{G} \\
\text { TDZ- } \\
\text { GTEA }\end{array}$ & $\begin{array}{c}\mathrm{AAXA}+\mathrm{GT} \\
\mathrm{DZ}\end{array}$ & $\mathrm{G}+\mathrm{Tr}-\mathrm{T}$ & GTEA & $\begin{array}{l}\text { Bean } \\
\text { (1987) } \\
\text { GTAH } \\
\end{array}$ & $\%$ & $\%$ & $\%$ & $\%$ & $\%$ \\
\hline
\end{tabular}




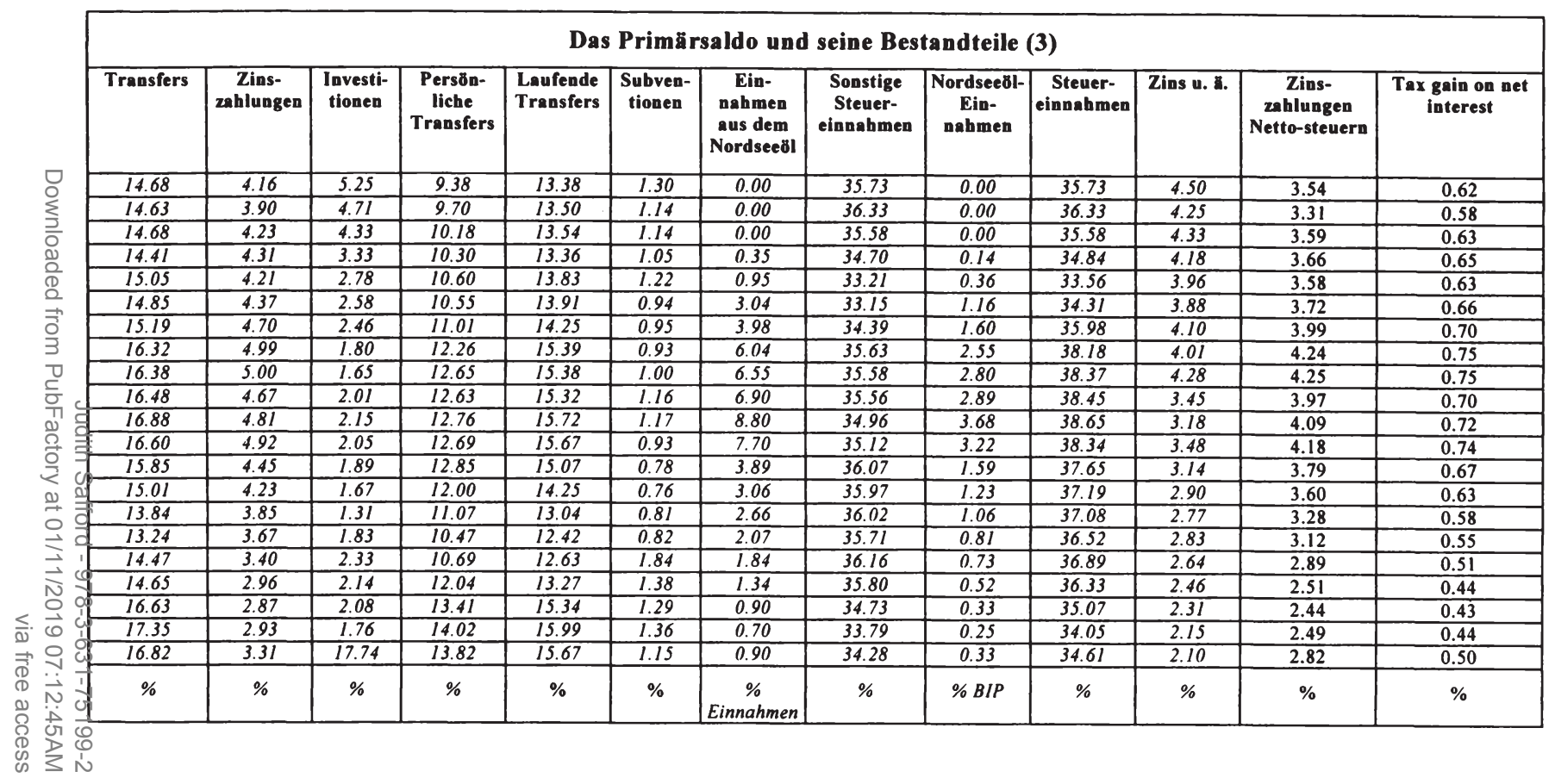


Die Ausgaben nach Aufgabenbereich (1)

\begin{tabular}{|c|c|c|c|c|c|c|c|c|c|c|c|}
\hline Jahr & $\begin{array}{c}\text { Offent. } \\
\text { Gater }\end{array}$ & $\begin{array}{l}\text { Verteidi- } \\
\text { gung }\end{array}$ & $\begin{array}{c}\text { Gesund- } \\
\text { heit }\end{array}$ & Blldung & $\begin{array}{c}\text { Soziale } \\
\text { Sicherung }\end{array}$ & $\begin{array}{c}\text { Wohnungs- } \\
\text { wesen }\end{array}$ & $\begin{array}{c}\text { Land- } \\
\text { wirtschaft }\end{array}$ & $\begin{array}{l}\text { Verkehrs- } \\
\text { wesen }\end{array}$ & $\begin{array}{l}\text { Energie, } \\
\text { Bergbau }\end{array}$ & $\underset{\text { BIP }}{\text { (Marktpreise) }}$ & Endkonsum \\
\hline 1979 & 5.7 & 4.5 & 5.2 & 5.2 & 10.6 & 3.7 & 0.6 & 1.7 & 1.8 & $198^{\prime} 221$ & 381883 \\
\hline 1980 & 5.6 & 4.9 & 5.7 & 5.5 & 11.0 & 3.6 & 0.7 & 1.5 & 1.7 & $231^{\prime} 772$ & $48^{\prime} 940$ \\
\hline 1981 & 5.7 & 5.0 & 5.9 & 5.6 & 12.2 & 2.8 & 0.6 & 1.6 & 1.5 & $254^{\prime} 927$ & $55^{\prime} 374$ \\
\hline 1982 & 5.6 & 5.2 & 5.7 & 5.5 & 13.0 & 2.4 & 0.7 & 1.8 & 1.3 & $279^{\prime} 041$ & $61^{\prime} 641$ \\
\hline 1983 & 5.5 & 5.2 & 5.9 & 5.4 & 12.9 & 2.4 & 0.8 & 1.7 & 1.1 & $304^{\prime} 456$ & $67^{\prime} 204$ \\
\hline 1984 & 5.8 & 5.3 & 5.8 & 5.2 & 13.0 & 2.4 & 0.8 & 1.1 & 1.1 & $325 ' 852$ & $71^{\prime} 201$ \\
\hline 1985 & 5.9 & 5.1 & 5.6 & 4.8 & 12.9 & 2.0 & 0.7 & 1.1 & 1.0 & $357^{\prime} 344$ & $75^{\prime} 267$ \\
\hline 1986 & 5.4 & 5.0 & 5.6 & 5.0 & 13.0 & 2.1 & 0.5 & 1.0 & 0.2 & $384^{\prime} 843$ & $80^{\prime} 911$ \\
\hline 1987 & 5.3 & 4.5 & 5.6 & 4.9 & 12.3 & 2.2 & 0.5 & 0.9 & -0.5 & $423^{\prime} 381$ & $87^{\prime} 045$ \\
\hline 1988 & 5.2 & 4.2 & 5.5 & 4.8 & 11.5 & 1.8 & 0.5 & 1.0 & -0.4 & $471^{\prime} 430$ & $93^{\prime} 641$ \\
\hline 1989 & 5.8 & 4.1 & 5.5 & 4.8 & 11.1 & 1.5 & 0.4 & 1.4 & -0.1 & 515957 & $101^{\prime} 796$ \\
\hline 1990 & 6.3 & 4.2 & 5.7 & 4.8 & 11.4 & 1.4 & 0.5 & 1.7 & -0.3 & $551^{\prime} 118$ & $112^{\prime} 934$ \\
\hline 1991 & 5.8 & 4.0 & 6.1 & 5.1 & 12.8 & 1.5 & 0.5 & 1.2 & -0.3 & $575^{\prime} 321$ & $124^{\prime} 105$ \\
\hline 1992 & 5.9 & 4.1 & 6.6 & 5.3 & 14.2 & 1.8 & 0.5 & 1.1 & 0.1 & $597 \cdot 242$ & $131 ' 875$ \\
\hline 1993 & 5.9 & 3.9 & 6.5 & 5.4 & 14.8 & 1.8 & 0.7 & 1.0 & 0.4 & $630^{\prime} 707$ & $137^{\prime} 965$ \\
\hline 1994 & 5.9 & 3.6 & 6.4 & 5.4 & 14.7 & 1.6 & 0.5 & 1.0 & 0.3 & $668 ' 866$ & $144^{\prime} 084$ \\
\hline & \begin{tabular}{|c|} 
Anteil am BIP \\
$(\%)$
\end{tabular} & $\begin{array}{c}\text { Anteil am } \\
\text { BIP (\%) }\end{array}$ & $\begin{array}{c}\text { Anteil am } \\
\text { BIP (\%) }\end{array}$ & $\begin{array}{c}\text { Anteil am } \\
\text { BIP (\%) }\end{array}$ & $\begin{array}{c}\text { Anteil am } \\
\text { BIP (\%) }\end{array}$ & $\begin{array}{c}\text { Anteil am } \\
\text { BIP (\%) }\end{array}$ & $\begin{array}{c}\text { Anteil am } \\
\text { BIP (\%) }\end{array}$ & $\begin{array}{c}\text { Anteil am } \\
\text { BIP (\%) }\end{array}$ & $\begin{array}{c}\text { Anteil am } \\
\text { BIP (\%) }\end{array}$ & Mrd. Pfund & Mrd. Pfund \\
\hline
\end{tabular}




\begin{tabular}{|c|c|c|c|c|c|c|c|c|c|c|c|}
\hline \multicolumn{12}{|c|}{ Die Ausgaben nach Aufgabenbereich (2) } \\
\hline \multirow{3}{*}{\begin{tabular}{|c|}
-35 \\
43 \\
\end{tabular}} & \multirow{2}{*}{\begin{tabular}{|c}
$\begin{array}{l}\text { Kapital- } \\
\text { bildung }\end{array}$ \\
$5^{\prime} 145$ \\
\end{tabular}} & \multirow{2}{*}{$\begin{array}{c}\begin{array}{c}\text { Ausgaben } \\
\text { (G) }\end{array} \\
43^{\prime} 993 \\
\end{array}$} & \multirow{2}{*}{$\begin{array}{c}\begin{array}{c}\text { offenth. } \\
\text { Gater }\end{array} \\
3 ' 710\end{array}$} & \multirow{2}{*}{\begin{tabular}{|c|} 
Verteidigung \\
$\mathbf{8}^{\prime} 969$ \\
\end{tabular}} & \multirow{2}{*}{$\begin{array}{c}\begin{array}{c}\text { Innere } \\
\text { Sicherheit }\end{array} \\
2 ' 893\end{array}$} & \multirow{2}{*}{$\begin{array}{c}\text { Bildung } \\
10^{\prime} 310\end{array}$} & \multirow{2}{*}{\begin{tabular}{|c|} 
Gesundheit \\
$9^{\prime} 082$ \\
\end{tabular}} & \multirow{2}{*}{$\begin{array}{c}\begin{array}{c}\text { Soziale } \\
\text { Sicherung }\end{array} \\
20^{\prime} 999\end{array}$} & \multirow{2}{*}{$\begin{array}{c}\begin{array}{c}\text { Wohnungs- } \\
\text { wesen }\end{array} \\
7^{\prime} 236 \\
\end{array}$} & \multirow{2}{*}{$\begin{array}{c}\begin{array}{c}\text { Sport } \\
\text { Erholung }\end{array} \\
1^{\prime} 138\end{array}$} & \multirow{2}{*}{$\begin{array}{c}\text { Energie } \\
1{ }^{\prime} 012 \\
\end{array}$} \\
\hline & & & & & & & & & & & \\
\hline & 5 '652 & $54^{\prime} 635$ & $4^{\prime} 318$ & $11^{\prime} 444$ & $3{ }^{\prime} 688$ & $12^{\prime} 752$ & $11{ }^{\prime} 633$ & $25^{\prime} 438$ & 81425 & $1 ' 463$ & $1 ' 295$ \\
\hline-93 & $4^{\prime} 672$ & $59^{\prime} 953$ & $4^{\prime} 603$ & $12^{\prime} 642$ & 4 '337 & $14^{\prime} 311$ & $13 \cdot 374$ & $31^{\prime} 107$ & $7 ' 105$ & $1 ' 569$ & 320 \\
\hline 155 & $4^{\prime} 437$ & $66^{\prime} 233$ & $4^{\prime} 946$ & $14^{\prime} 451$ & $4^{\prime} 867$ & $15^{\prime} 286$ & $14^{\prime} 081$ & $36^{\prime} 413$ & $6 ' 560$ & $1^{\prime} 688$ & 761 \\
\hline 246 & $5 ' 869$ & $73^{\prime} 319$ & $5 ' 130$ & $15 ' 840$ & 5,326 & $16^{\prime} 329$ & $15^{\prime} 928$ & $39^{\prime} 296$ & $7^{\prime} 422$ & $1^{\prime} 960$ & 523 \\
\hline 280 & 6'719 & $78^{\prime} 200$ & $5^{\prime} 462$ & $17^{\prime} 133$ & $5^{\prime} 943$ & $16^{\prime} 903$ & $16^{\prime} 781$ & $42^{\prime} 213$ & $7^{\prime} 963$ & $2^{\prime} 136$ & $1^{\prime 2} 238$ \\
\hline 450 & $6 ' 872$ & 82.589 & 6'013 & $18^{\prime} 213$ & 6.225 & $17^{\prime} 283$ & $17^{\prime} 889$ & $46^{\prime} 246$ & $7^{\prime} 008$ & $2 ' 241$ & $1 ' 079$ \\
\hline-237 & $7 ' 509$ & $88^{\prime} 183$ & $66^{\prime} 326$ & $19^{\prime} 066$ & $66^{\prime} 826$ & $19^{\prime} 271$ & $19^{\prime} 164$ & $49^{\prime} 947$ & $8^{\prime} 066$ & $2^{\prime} 446$ & $-1^{\prime} 174$ \\
\hline-498 & 7'577 & $94^{\prime} 124$ & 6.531 & $18^{\prime} 943$ & $7 ' 524$ & $20^{\prime} 829$ & $20^{\prime} 905$ & $52^{\prime} 133$ & $9^{\prime} 227$ & 2 '599 & $-3^{\prime} 370$ \\
\hline-322 & 6'506 & $99 ' 825$ & 7.595 & $19^{\prime} 797$ & $8^{\prime} 406$ & $22 ' 578$ & $22^{\prime} 936$ & $54^{\prime} 257$ & $8^{\prime} 495$ & $2^{\prime} 828$ & $-3^{\prime} 026$ \\
\hline-163 & $9^{\prime} 582$ & $111^{\prime 215}$ & $9^{\prime} 336$ & $20^{\prime} 988$ & $9^{\prime} 433$ & $24 ' 837$ & $25^{\prime} 152$ & $57^{\prime} 343$ & $\begin{array}{l}7996 \\
\end{array}$ & $3 ' 185$ & $-1^{\prime} 785$ \\
\hline 156 & $12^{\prime} 659$ & $125^{\prime} 749$ & $10^{\prime} 639$ & $22^{\prime} 907$ & $10^{\prime} 932$ & $26^{\prime} 724$ & $27^{\prime} 766$ & $62^{\prime} 928$ & $7 ' 799$ & 3'655 & $-3^{\prime} 016$ \\
\hline 151 & $12^{\prime} 143$ & $1366^{\prime} 399$ & $11 ' 517$ & $23^{\prime} 226$ & $12^{\prime} 923$ & 29,355 & $31 ' 150$ & 73 '795 & $8^{\prime} 695$ & $3^{\prime} 914$ & $-3^{\prime} 054$ \\
\hline-17 & $12^{\prime} 506$ & $144^{\prime} 364$ & $11^{\prime} 960$ & $24^{\prime} 402$ & $12^{\prime} 854$ & $31^{\prime} 947$ & 34 '999 & $84^{\prime} 666$ & $10^{\prime} 604$ & 4'168 & -516 \\
\hline-24 & $11^{\prime} 487$ & $149^{\prime} 428$ & $11^{\prime} 966$ & $24^{\prime} 561$ & $14^{\prime} 434$ & $33^{\prime} 805$ & $36^{\prime} 764$ & $93^{\prime} 483$ & $111^{\prime} 083$ & $4^{\prime} 211$ & $1 ' 127$ \\
\hline-250 & $12^{\prime} 625$ & 156.459 & $12^{\prime} 681$ & $23 \cdot 815$ & $15^{\prime} 049$ & $36^{\prime} 057$ & $38^{\prime} 657$ & $98^{\prime} 236$ & $10^{\prime} 591$ & $4^{\prime} 476$ & 600 \\
\hline $\begin{array}{l}\text { Mrd. } \\
\text { Pfund }\end{array}$ & $\begin{array}{c}\text { Mrd. } \\
\text { Pfund }\end{array}$ & Mrd. Pfund & Mrd. Pfund & Mrd. Pfund & Mrd. Pfund & Mrd. Pfund & Mrd. Pfund & Mrd. Pfund & Mrd. Pfund & Mrd. Pfund & Mrd. Pfund \\
\hline
\end{tabular}




\section{Die Ausgaben nach Aufgabenbereich (3)}

\begin{tabular}{|c|c|c|c|c|c|c|c|c|c|}
\hline \begin{tabular}{|c|} 
Land, \\
Forstwirtschaft \\
,Fischerei \\
\end{tabular} & Erdbau,etc & $\begin{array}{l}\text { Verkehrs u } \\
\text { Nachrichten- } \\
\text { wesen }\end{array}$ & $\begin{array}{c}\text { Sonstige } \\
\text { סkon. } \\
\text { Dienste }\end{array}$ & Sonstige & $\begin{array}{c}\text { Offentl. } \\
\text { Guter }\end{array}$ & $\begin{array}{c}\text { Gesundheit, } \\
\text { Sport }\end{array}$ & $\begin{array}{l}\text { Erdbau, } \\
\text { Energie }\end{array}$ & $\begin{array}{c}\text { Zins- } \\
\text { zahlungen }\end{array}$ & Sonstige \\
\hline $1 ' 163$ & $2 ' 508$ & 3,380 & $1 ' 883$ & $11{ }^{\prime} 409$ & $11 \cdot 226$ & $10^{\prime} 220$ & $3 ' 520$ & $8^{\prime} 669$ & $2 ' 740$ \\
\hline 1'625 & $2 ' 716$ & $3 ' 568$ & 2425 & $13^{\prime} 410$ & $12^{\prime} 953$ & 13.096 & $4^{\prime} 011$ & $10^{\prime} 888$ & $2 ' 522$ \\
\hline 1'655 & $3 ' 564$ & 4'199 & $2^{\prime} 930$ & $15^{\prime} 353$ & $14^{\prime} 511$ & $14^{\prime} 943$ & $3 ' 884$ & $12^{\prime} 712$ & $2 ' 641$ \\
\hline $2^{\prime} 041$ & $2 ' 811$ & $5^{\prime} 067$ & $2^{\prime} 886$ & $16 ' 897$ & $15^{\prime} 644$ & $15^{\prime} 769$ & 3.572 & $13^{\prime} 952$ & 2'945 \\
\hline 2,346 & $2 ' 734$ & $5 ' 080$ & 3'328 & $17^{\prime} 227$ & $16 \cdot 803$ & $17^{\prime} 888$ & 3257 & $14^{\prime} 208$ & 3'019 \\
\hline $2 ' 538$ & $2 ' 396$ & 3480 & $4 ' 230$ & $18^{\prime} 802$ & $18^{\prime} 767$ & $18^{\prime} 917$ & 3'634 & 151670 & $3^{\prime} 132$ \\
\hline $2^{\prime} 622$ & $2 ' 375$ & 4'094 & $4^{\prime} 348$ & $22^{\prime} 128$ & $21^{\prime} 128$ & $20^{\prime} 130$ & 34454 & $17^{\prime} 586$ & 4'542 \\
\hline $2^{\prime} 088$ & 1'906 & $3^{\prime} 728$ & $4^{\prime} 128$ & $20^{\prime} 551$ & $20^{\prime} 680$ & $21^{\prime} 610$ & 732 & $17^{\prime} 151$ & 3,400 \\
\hline $2 ' 299$ & $1 ' 274$ & $3 ' 998$ & $3 ' 714$ & $22^{\prime} 635$ & $22^{\prime} 468$ & $23 ' 504$ & $-2{ }^{\prime} 096$ & $17^{\prime} 936$ & $4 ' 699$ \\
\hline $2 ' 487$ & 917 & $4 ' 480$ & 3'572 & $22^{\prime} 915$ & $24^{\prime} 291$ & $25^{\prime} 764$ & $-2^{\prime} 109$ & $18^{\prime} 197$ & $4 ' 718$ \\
\hline $1 ' 972$ & $1 ' 524$ & $7 \cdot 129$ & $5 ' 049$ & $24^{\prime} 927$ & $29 ' 818$ & $28 \cdot 337$ & -261 & $18^{\prime} 927$ & $6^{\prime} 000$ \\
\hline $2 ' 647$ & $1 ' 270$ & $9 ' 443$ & $6 ' 727$ & $25^{\prime} 127$ & $34^{\prime} 679$ & $31^{\prime} 421$ & $-1 ' 746$ & $18^{\prime} 746$ & 6'381 \\
\hline $2^{\prime} 906$ & 1'544 & $6 ' 738$ & $4^{\prime} 154$ & $21 ' 544$ & $33^{\prime} 134$ & $35^{\prime} 064$ & $-1 ' 510$ & $17^{\prime} 004$ & $4^{\prime} 540$ \\
\hline 3'111 & $1 ' 222$ & $6^{\prime} 644$ & 4488 & 231055 & 35219 & $39^{\prime} 167$ & 706 & $17^{\prime} 138$ & $5 ' 917$ \\
\hline $4^{\prime} 161$ & $1 ' 337$ & 6.576 & $5 ' 228$ & $24^{\prime} 377$ & $37^{\prime} 495$ & $40^{\prime} 975$ & $2^{\prime} 464$ & $18^{\prime} 510$ & $5 ' 867$ \\
\hline $3 ' 280$ & $1 ' 632$ & $6 ' 441$ & $6 \cdot 320$ & $27^{\prime} 901$ & $39^{\prime} 782$ & $43^{\prime} 133$ & $2 ' 232$ & $22^{\prime} 169$ & $5 ' 732$ \\
\hline Mrd. Pfund & Mrd. Pfund & Mrd. Pfund & Mrd. Pfund & Mrd. Pfund & Mrd. Pfund & Mrd. Pfund & Mrd. Pfund & Mrd. Pfund & Mrd. Pfund \\
\hline
\end{tabular}




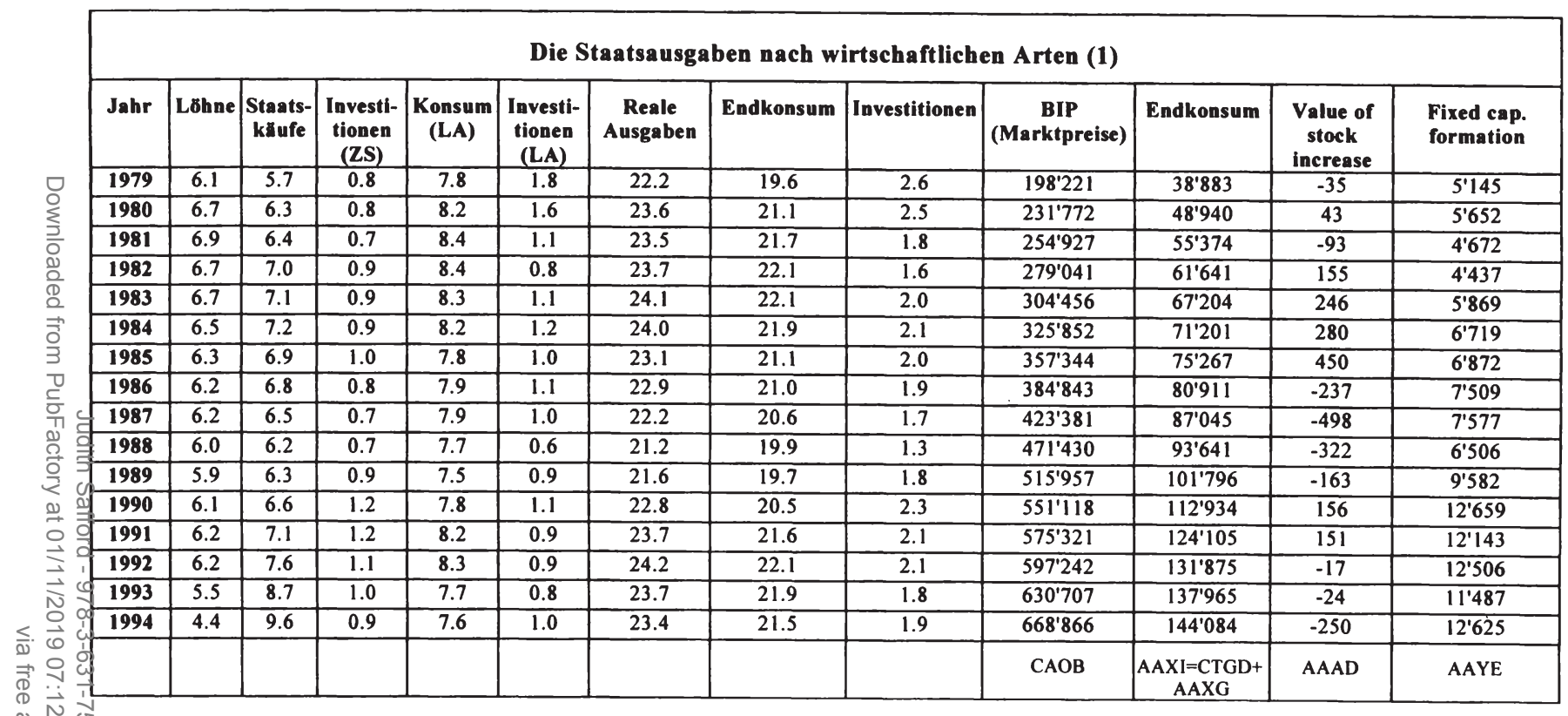


Die Staatsausgaben nach wirtschaftlichen Arten (2)

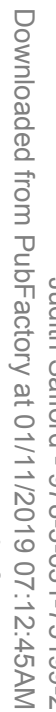

\begin{tabular}{|c|c|c|c|c|c|c|c|c|c|}
\hline \multicolumn{10}{|c|}{ Die Staatsausgaben nach wirtschaftlichen Arten (2) } \\
\hline $\begin{array}{l}\text { Ausgaben } \\
\text { (G) }\end{array}$ & $\begin{array}{l}\text { Lohne und } \\
\text { Gehalte }\end{array}$ & Staatskäufe & $\begin{array}{l}\text { Kapital- } \\
\text { konsum }\end{array}$ & $\begin{array}{l}\text { Subventionen } \\
\text { f. Bildung }\end{array}$ & $\begin{array}{c}\text { Staatskăufe, } \\
\text { etc. }\end{array}$ & $\begin{array}{l}\text { Endkonsum } \\
\text { Zentralstaat }\end{array}$ & Kapital ZS & Endkonsum LA & Kapital LA \\
\hline $43^{\prime} 993$ & $12^{\prime} 028$ & & & & $11^{\prime} 373$ & $23^{\prime} 401$ & $1 ' 522$ & $15^{\prime} 482$ & $3^{\prime} 588$ \\
\hline $54^{\prime} 635$ & $15^{\prime} 460$ & & & & $14 ' 529$ & $29^{\prime} 989$ & $1 ' 800$ & $18^{\prime} 951$ & $3^{\prime} 742$ \\
\hline $59 ' 953$ & $17^{\prime} 481$ & & & & $16 ! 398$ & $33 ' 879$ & $1 ' 770$ & $21 ' 495$ & $2 ' 715$ \\
\hline $66^{\prime} 233$ & $18^{\prime} 617$ & & & & $19 ' 661$ & $38^{\prime} 278$ & 2,385 & $23 ' 363$ & $2 ' 207$ \\
\hline $73 ' 319$ & $20^{\prime} 428$ & & & & $21^{\prime} 643$ & $42^{\prime} 071$ & $2^{\prime} 743$ & $25^{\prime} 133$ & 3,372 \\
\hline $78^{\prime} 200$ & $21 ' 243$ & $21^{\prime} 003$ & 896 & $1^{\prime} 441$ & $23 ' 340$ & $44^{\prime} 583$ & $3^{\prime} 008$ & 26,618 & $3^{\prime} 991$ \\
\hline $82 ' 589$ & $22^{\prime} 656$ & $222^{\prime} 240$ & 983 & $1 ' 462$ & $24^{\prime} 685$ & $47^{\prime} 341$ & 31576 & $27^{\prime} 926$ & 31746 \\
\hline $88^{\prime} 183$ & $23^{\prime} 971$ & $23^{\prime} 754$ & $1 ' 076$ & $1 ' 530$ & $26^{\prime} 360$ & $50^{\prime} 331$ & $3^{\prime} 114$ & $30^{\prime} 580$ & $4^{\prime} 158$ \\
\hline $94^{\prime} 124$ & $26^{\prime} 131$ & $24^{\prime} 730$ & $1 ' 179$ & $1^{\prime} 696$ & $27 ' 605$ & $53^{\prime} 736$ & $2^{\prime} 860$ & $33^{\prime} 309$ & $4^{\prime} 219$ \\
\hline $99^{\prime} 825$ & $28^{\prime} 105$ & $26 ' 202$ & $1 ' 303$ & $1 ' 912$ & $29^{\prime} 417$ & $57^{\prime} 522$ & 3,387 & $36 ' 119$ & $2 ' 797$ \\
\hline 111215 & $30^{\prime} 698$ & $28^{\prime} 345$ & $l^{\prime} 484$ & $2 ' 767$ & $32 ' 596$ & 63'294 & $4^{\prime} 788$ & $38^{\prime} 502$ & $4^{\prime} 631$ \\
\hline $125^{\prime} 749$ & $33^{\prime} 777$ & $31 ' 652$ & $1 ' 623$ & $3^{\prime} 056$ & 36,331 & $70^{\prime} 108$ & $6 ! 571$ & $42^{\prime} 826$ & $6 ' 244$ \\
\hline $136^{\prime} 399$ & $35^{\prime} 951$ & $36 ' 605$ & I'675 & $2^{\prime} 754$ & $41^{\prime} 034$ & $76^{\prime} 985$ & 7'027 & $47^{\prime} 120$ & $5 ' 267$ \\
\hline $144^{\prime} 364$ & $36^{\prime} 989$ & $40^{\prime} 531$ & $1 ' 608$ & 3,131 & $45^{\prime} 270$ & $82 ' 259$ & 61838 & $49^{\prime} 616$ & 51651 \\
\hline $149^{\prime} 428$ & $34^{\prime} 420$ & $47^{\prime} 186$ & $1 ' 592$ & $5^{\prime} 920$ & $54^{\prime} 698$ & $89^{\prime} 118$ & 6,518 & $48 ' 847$ & $4^{\prime} 945$ \\
\hline $156^{\prime} 459$ & $29^{\prime} 242$ & $55^{\prime} 371$ & $1 ' 408$ & $7 ' 393$ & $64^{\prime} 172$ & $93 \cdot 414$ & $5 ' 860$ & $50^{\prime} 670$ & 6,515 \\
\hline $\begin{array}{l}\text { AAXI+AAYE } \\
+\mathrm{AAAD}=\mathrm{gteg} \\
\end{array}$ & GILN & CUCN & ACGL & CUKF & achc-giln & $\begin{array}{c}\mathrm{ACHC}(+\mathrm{csba}= \\
\text { aaxi })\end{array}$ & gteb & CSBA & AAAG \\
\hline
\end{tabular}




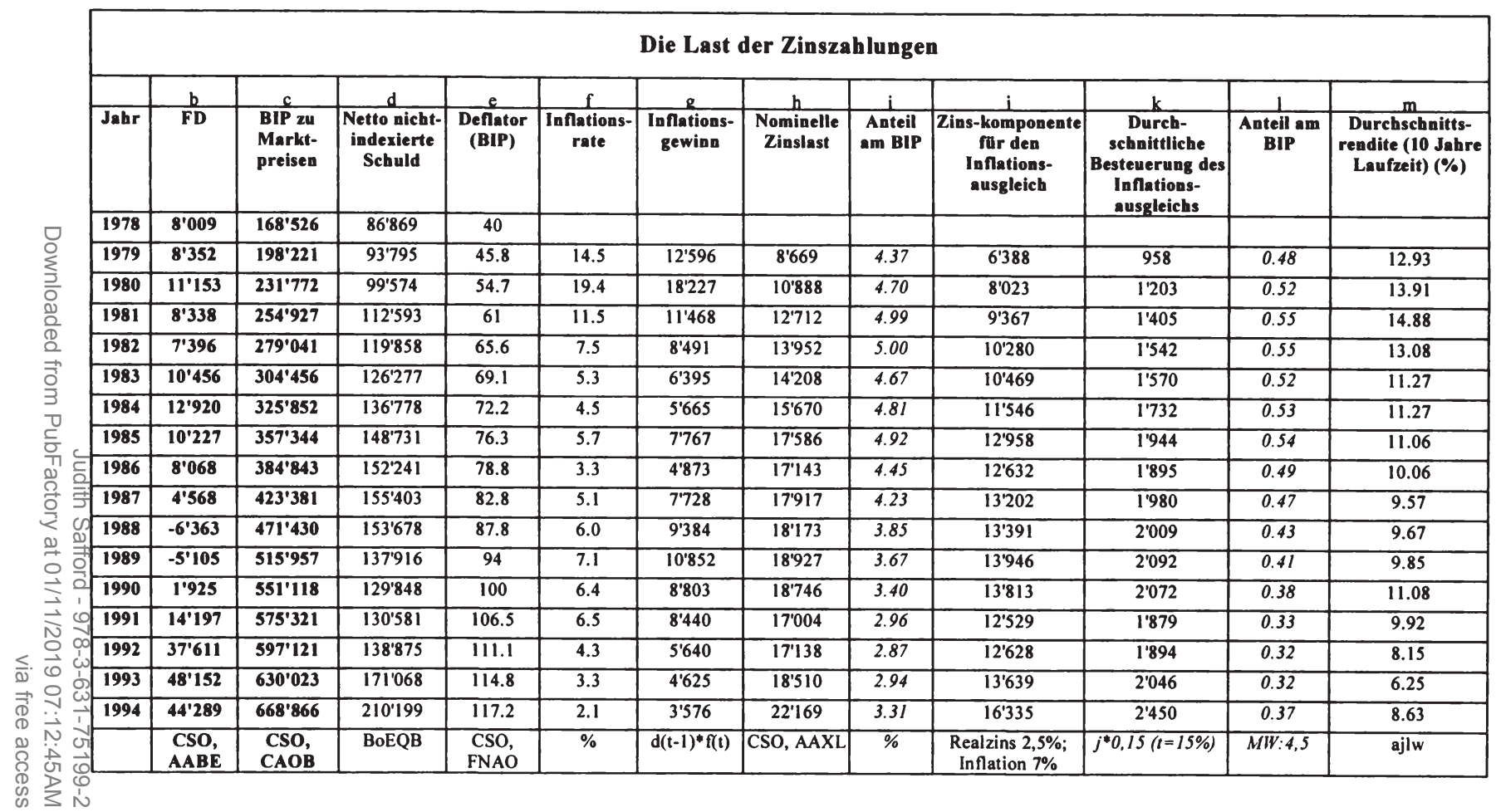




\section{Literaturverzeichnis}

Abel, A. B. (1986): The Failure of Ricardian Equivalence under progressive wealth taxation, in: Journal of Public Economics, Vol. 30, Nr. 1, S. 117-128

Abel, A. B., Mankiw, N. G., Summers, L. H., Zeckhauser, R. J. (1989): Assessing Dynamic Efficiency: Theory and Evidence, in: Review of Economic Studies, Vol. 56, S. 1-19

Aghion, P. und Bolton, P. (1989): Government domestic debt and the risk of default: a politicaleconomic model of the strategic role of debt, in: Dornbusch, R. und Draghi, M. (Hrsg.), Public Debt Management: theory and history, Cambridge, UK, S. 313-347

Atkinson, A. B. (1983): The Economics of Inequality, 2. Aufl., London

Atkinson, A. B. und Stiglitz, J. E. (1980): Lectures on Public Economics, London

Alesina, A. (1988): The End of Large Public Debts, in: F. Giavazzi und L. Spaventa (Hrsg.) High Public Debt: The Italien Experience, Cambridge, S. 34-79

Alesina, A. (1992): Political Models of Macroeconomic Policy and Fiscal Models, World Bank Working Paper WPS 970

Alesina, A. und Drazen, A. (1991): Why Are Stabilizations Delayed?, in: American Economic Review, Vol. 81, S. 1170-1188

Alesina, A. und Perotti, R. (1994): The Political Economy of Budget Deficits, NBER Working Paper Nr. 4637

Alesina, A. und Perotti, R. (1996): Budget Deficits and Budget Institutions, NBER Working Paper Nr. 5556

Alesina, A. und Tabellini, G. (1990): A Positive Theory of Fiscal Deficits and Government Debt, in: Review of Economic Studies, Vol. 57, S. 403-414

Andel, N. (1990): Finanzwissenschaft, 2. Aufl., Tübingen

Arrow, K. J. und Lind, R. C. (1970): Uncertainty and the Evaluation of Public Investment, in: American Economic Review, Vol. 60, Nr. 3, S. 364-378

Artis, M. (1988): The 1988 Budget and the MTFS, in: Fiscal Studies, Vol. 9, Nr. 2, S. 14-29

Ashby, P. (1985): The Long-Term Unemployed: Action for a forgotten million, a NCVO discussion document, London

Ashworth, M. J. (1984), What are Public Assets Worth?, in: Ashworth, M., J. Hills und N. Morris (1984) Public Finances in Perspective, IFS Report Series Nr. 8, London, S. 50-56

Auerbach, A. J., Gokhale, J. und Kotlikoff, L. J. (1991): Generational Accounts - a meaningful alternative to deficit accounting, NBER Working Paper Nr. 3589

Auerbach, A. J. und Kotlikoff, L. J. (1987): Dynamic Fiscal Policy, Cambridge

Bacon, R. und Eltis, W. (1976): Britain's Economic Problem: too few producers, London

Bank of England, Quarterly Bulletin, verschiedene Jahrgänge

Barrell, R. J., Morgan, J., Sefton, J. und in't Veld, J. (1994): The Cyclical Adjustment of Budget Balances, in: NIESR Report Series Nr. 8

Barro, R. J. (1974): Are Government Bonds Net Wealth?, in: Journal of Political Economy, Vol. 82 , Nr. 6, S. $1095-1117$

Barro, R. J. (1979): On the Determination of the Public Debt, in: Journal of Political Economy, Vol. 87, Nr. 3, S. 940-71 
Barro, R.J. (1987): Government spending, interest rates, prices, and budget deficits in the United Kingdom, 1701-1918, in: Journal of Monetary Economics, 20, S. 221-247

Barro, R. J. (1989): The Ricardian Approach to Budget Deficits, in: Journal of Economic Perspectives, Vol. 3, Nr. 2, S. 37-54

Barro, R. J. und Grilli, V. (1994): European Macroeconomics, London

Barro, R. J. und Sala i Martin, X. (1995): Economic Growth, New York

Baumol, W. J. (1967): Macroeconomics of Unbalanced Growth: The Anatomy of Urban Crisis, in: American Economic Review, Vol. 57, Nr. 3, S. 415-426.

Bean, C. (1987): The Impact of North Sea Oil, in: Dornbusch, R. und Layard, P. R. G. (Hrsg.) The Performance of the British Economy, Oxford, S. 64-96

Becker, G. S. (1981): A treatise on the family, Cambridge, MA

Becker, G. S. (1983): A theory of competition amongst pressure groups for political influence, in: The Quarterly Journal of Economics, Nr. 98, S. 371-400

Becker, G. S. (1985): Public Policies, Pressure Groups and Dead Weight Costs, in: Journal of Public Economics, Vol. 28, S. 329-347

Becker, W. (1990): Determinanten eines Wachstums der Staatsausgabenquote, Studien zur Wirtschafts- und Finanzwissenschaft Bd 2, Bergisch Gladbach

Begg, D. K. H. (1982): The Rational Expectations Revolution in Macroeconomics: Theories and Evidence, Oxford

Begg, D. K. H. (1987): Fiscal Policy, in: Dornbusch, R. und Layard, R. (Hrsg.), The Performance of the British Economy, Oxford, S. 29-63

Bernheim, B. D. (1987): Ricardian Equivalence: An Evaluation of Theory and Evidence, NBER Working Paper Nr. 2330, Cambridge, MA

Bernheim, B. D., Shleifer, A. und Summers, L. (1985): The Strategic Bequest Motive, in: Journal of Political Economy, Vol. 93, Nr. 6, S. 1045-1076

Blanchard, O. (1981): Output, the Stock Market and Interest Rates, in: American Economic Review, 71, 1 S. 132-143

Blanchard, O. J. (1985): Debt, Deficits, and Finite Horizons, in: Journal of Political Economy, Vol. 93, S. 223-247

Blanchard, O. (1990): Suggestions for a New Set of Fiscal Indicators, OECD Department of Economics and Statistics, Working Paper No. 79

Blanchard, O. und Dornbusch, R. (1986a): US Deficits, the Dollar, and Europe, in: Blanchard, O, R. Dornbusch und R. Layard, Restoring Europe's Prosperity, Cambridge, MA, S. 156179

Blanchard, O., Dornbusch, R.und Buiter, W. (1986b): Public Debt and Fiscal Responsibility, in: Blanchard, O, R. Dornbusch und R. Layard, Restoring Europe's Prosperity, Cambridge, MA, S. 125-153

Blankart, C. (1985): Zwei Wege zur Privatisierung öffentlicher Dienstleistungen, München

Blankart, C. (1994): Öffentliche Finanzen in der Demokratie, 2. Aufl., München

Blinder, A. S. (1981): Thoughts on the Laffer Curve, in: The Supply-Side Effects of Economic Policy, Meyer, L. H. (Hrsg.), Hingham, MA, S. 81-92

Blinder, A. S. und R. M. Solow (1973): Does Fiscal Policy Matter?, in: Journal of Public Economics, 2, S. 319-337 
Blinder, A. S. und R. M. Solow (1974): Analytical Foundations of Fiscal Policy, in: Blinder, A. S. et al. (Hrsg.), The Eonomics of Public Finance, Washington

Blinder, A. S. und R. M. Solow (1976): Does Fiscal Policy Matter? A Correction, in: Journal of Public Economics 5, S. 183-4

Blundell, R. W. (1992): Labour supply and taxation: A survey, in: Fiscal Studies, Vol. 13(3), S. $15-40$

Blundell, R. W. und Walker, I. (1986): A life-cycle consistent empirical model of labour supply using cross section data, in: Review of Economic Studies, Vol. 53, S. 539-558

Blundell, R. W., Ham, J. und Meghir, C. H. D. (1987): Unemployment and female labour supply, in: Economic Journal, Vol. 97, S. 44-64

Bodnador, V. (Hrsg.) (1996): Politics and the Constitution. Essays on British Government, Oxford

Bohn, H. (1992): Endogenous government spending and Ricardian equivalence, in: Economic Journal, Vol. 102, S. 588-597

Boll, S., Raffelhüschen, B. und Walliser, J. (1994): Social Security and Intergenerational Redistribution: A Generational Accounting Perspective, in: Public Choice, Vol. 81, Nr. 1-2, S. 79-100

Borcherding, T. E., Pommerehe, W. W. und Schneider, F. (1982): Comparing the Efficiency of Private and Public Production: The Evidence from Five Countries in: Bös, D., Musgrave, R.A. und Wiseman, J. (Hrsg.) Public Production, Zeitschrift für Nationalökonomie, Supplement 2, Wien, S. 127-156

Borooah, V. K. (1988): The Growth of Public Expenditure in the United Kingdom, 1960-1986, in: Lybeck, J. A. und Henrekson, M. (Hrsg.) Explaining the Growth of Government, Amsterdam, S. 299-326

Bös, D. (1982) Crisis of the Tax State, in: Public Choice, Vol. 38, S. 225-241

Bös, D. (1989): Arguments on Privatisation, in: Fels, G. und v. Furstenberg, G. M. (Hrsg.) A Supply-side Agenda for Germany, Berlin, S. 217-246

Bös, D. (1991): Privatization: A theoretical treatment, Oxford

Bovenberg, A. L. und Horne, J. P. (1992): Taxes on Commodities: A Survey, in: Kopits, G. (Hrsg) (1992): Tax Harmonization in the European Community. Policy Issues and Analysis, International Monetary Fund Occasional Paper Nr. 94

Bradshaw, J. und Huby, M. (1989): Trends in Dependence on Supplementary Benefit, in: The Economics of Social Security, Dilnot, A. und Walker, I. (Hrsg.), Oxford, S. 69-87

Brennan, G. und Buchanan, J. (1979): The logic of tax limits: alternative constitutional constraints on the power to tax, in: National Tax Journal: Supplement, S. 11-22

Brittan, S. (1983): The Role and Limits of Government: Essays in Political Economy, London

Britton, A. (1991): Macroeconomic Policy in Britain 1974-87, Cambridge, UK

Brook, L. und Cape, E. (1995): Libertarianism in retreat? , in: Jowell, R., Curtice, J., Park, A., Brook, L. und Ahrendt, D. (Hrsg.), British Social Attitudes, the 12th Report, S. 191-209

Buchanan, J. M. (1958): Concerning Future Generations, wieder abgedruckt in: Ferguson, J. M. (Hrsg.) (1964): Public Debt and Future Generations, Chapel Hill, S. 55-63

Buchanan, J. M. (1975): The Limits of Liberty: Between Anarchy and Leviathan, Chicago

Buchanan, J. M. (1976): Barro on the Ricardian Equivalence Theorem, in: Journal of Political Economy, Vol. 84, S. 337-342 
Buchanan, J. M. und Tullock, G. (1967): The Calculus of Consent. Logical Foundations of Constitutional Democracy, Michigan

Buchanan, J. M. und Wagner, R. E. (1977): Democracy in Deficit. The Political Legacy of Lord Keynes, New York

Buchanan, J., Rowley, C. und Tollison, R. (1987): Deficits, Oxford

Budd, A. (1979): Disarming the Treasury, in: The Taming of Government, Institute of Economic Affairs, IEA Readings Nr. 21, London, S. 91-106

Budd, A. (1985): Macroeconomic Policy and the 1985 Budget, in: Fiscal Studies, Vol. 6, Nr. 2, S. $10-22$

Budd, A. (1988): Macro-economic Aspects of the 1988 Budget, in: Fiscal Studies, Vol. 9, Nr. 2, S. 1-13

Budd, A. und Burns, T. (1981): The Relationship between Fiscal and Monetary Policy in the London Business Modell, in: Artis, M. J. und Miller, M. H. (Hrsg.), Essays in Fiscal and Monetary Policy, Oxford

Budd, A. Dicks, G und Keating, G. (1985): Economic Viewpoint, Fiscal Policy and the Problem of Unemployment, in: Economic Outlook, London Business School, Juni, S. 22-32

Buiter, W. H. (1977): „Crowding out“ and the effectiveness of fiscal policy“ in: Journal of Public Economics, Vol. 7, S. 309-328

Buiter, W. H. (1983): The Theory of Optimum Deficits and Debts, Centre for Labour Economics, London School of Economics, Discussion Paper Nr. 177

Buiter, W. H. (1985): A guide to public sector debt and deficits, in: Economic Policy, Vol. 5, S. 15-79

Buiter, W. H. (1990): Debt Neutrality, Redistribution, and Consumer Heterogeneity: A Survey and Some Extensions, S. 79-114 in: Brainard, W., W. Nordhaus und H. Watts (Hrsg.), Macroeconomics, Finance and Economic Policy; Essays in Honor of James Tobin, Cambridge, MA., S. 79-114

Buiter, W. H. (1993): Measurement of the Public Sector Deficit and Its Implications for Policy Evaluation and Design, in: Blejer, M. I. und Cheasty, A. (Hrsg.) How to measure the fiscal deficit, Washington, S. 297-344

Buiter, W. H. und Miller, M. (1981): The Thatcher Experiment: The First Two Years, in: Brookings Papers on Economic Activity, Vol. 8, S. 315-367

Buiter, W. H. und Miller, M. (1983): Changing the Rules: Economic Consequences of the Thatcher Regime, in: Brookings Papers on Economic Activity, Vol. 10, S. 305-365

Buiter, W. H. und J. Tobin (1981): Debt Neutrality: A Brief Review of Doctrine and Evidence, in: von Furstenberg, G. M. (Hrsg.), Social Security versus Private Saving, Cambridge, MA, S. 39-63

Buiter, W. H. und J. Tobin ([1980] wieder abgedruckt 1990): „Fiscal and monetary policies, capital formations, and economic activity“, in: Buiter, W. H. (1990): Principles of Budgetary and Financial Policy, S. 287-356.

Buiter, W. H. und Kletzer, K. M. (1991): Reflections on the fiscal implications of a common currency, in: Giovannini, A. und Mayer, C. (Hrsg.) European financial integration, Cambridge, S. 221-251

Bunz, A. R. (Hrsg.) (wird laufend aktualisiert): Euro Guide. Der Europäische Markt von A-Z, Köln

Burk, K. und Cairncross, A. (1992) Goodbye, Great Britain, Yale, New Haven 
Burton, J. (1985): Why No Cuts. An Inquiry into the Fiscal Anarchy of Uncontrolled Government Expenditure, Hobart Paper Nr. 104

Byatt, I. (1989): The Record of Tax Reform in Great Britain and its Contribution to the Supply Side, in: Fels, G. und v. Furstenberg, G. M. (Hrsg.) A Supply-side Agenda for Germany, Berlin, S. 355-377

Cairncross, A. (1992): The British Economy since 1945, Oxford

Cantor, V. A., Joines, D. H. und Laffer, A. B. (1981): Tax Rates, Factor Employment, and Market Production, S. 3-32, in: The Supply-Side Effects of Economic Policy, Meyer, L. H. (Hrsg.), Hingham, MA

Carlberg, M. (1988): Public Debt, Taxation and Government Expenditures in a Growing Economy, Berlin

Carmichael, J. (1979): The Role of Government Financial Policy in Economic Growth, Ph.D. dissertation, Princeton University

Cassel, D. (1982): Staatsverschuldung international. Stand und Entwicklung der öffentlichen Kreditaufnahme ausgewählter OECD-Länder im Vergleich, S. 667-707, in: Möglichkeiten und Grenzen der Staatstätigkeit, Bombach, G., Gahlen, B. und Ott, A. E. (Hrsg.), Tübingen

Cecco, de M. und Giovannini, A. (Hrsg.) (1989) A European Central Bank? Perspectives on monetary unification after ten years of the EMS, Cambridge

Christ, C. F. (1967): A Short-run aggregate-demand model of the Interdependence and effects of monetary and fiscal policies with Keynesian and classical interest elasticities, in: American Economic Review, Vol. LVII, May, S. 434-443

Christ, C. F. (1968): A Simple Macroeconomic Model with a Government Budget Restraint, in: Journal of Political Economy, Vol. 76, Nr. 1, S. 53-67

Christ, C. F. (1979): On Fiscal and Monetary Policies and the Government Budget Restraint, in: American Economic Review, Vol. 69 S. 526-539

Coase, R. H. (1937): The Nature of the Firm, in: Economica, Vol. 4, S. 386-405

Conservative Central Office (1977): The Right Approach to the Economy, London

Conservative Central Office (1987): Conservative Party Manifesto, London

Conservative Central Office (1991): Conservative Party Manifesto, London

Crawford, I. und Tanner, S. (1995): Bringing It All Back Home: Alcohol Taxation and CrossBorder Shopping, in: Fiscal Studies, Vol. 16(2), S. 94-114

Crafts, N. (1988): The Assessment: British Economic Growth over the long run, in: Oxford Review of Economic Policy, Vol. 4 Nr. 1, S. i-xviii

Crewe, I, Day, N. und Fox, A. (1991): The British Electorate 1963-1987, Cambridge

Cross, R. B. und Shaw, G. K. (1982): On the Economics of Tax Aversion, in: Public Finance Vol. 37, Nr. 1, S. 36-47

CSO Economic Trends, verschiedene Monatsausgaben

CSO Financial Statistics, verschiedene Monatsausgaben

CSO National Income and Accounts „Blue Book“, verschiedene Jahrgänge

CSO (1993): A new approach to controlling public expenditure, in: Treasury Bulletin, Vol. 4 (2) S. 11-18.

Cukierman, A. (1986): Uncertain Lifetimes and the Ricardian Equivalence Proposition, Department of Economics, Tel-Aviv University, Working Paper Nr. 45-86 
Cukierman, A., Lennan, K. und Papadia, F. (1985): Inflation induced redistribution via monetary assets in five European countries: 1974-1982, Economic Paper of the Commission of the European Communities, Nr. 41

Cukierman, A. und Meltzer, A. H. (1989): A Political Theory of Government Debt and Deficits in a Neo Ricardian Framework, in: American Economic Review, Vol. 79, Nr. 4. S. 713732

Cullis, J. G. und Jones, P. R. (1992): Public Finance and Public Choice. Analytical perspectives, London

Cullis, J. G. und Jones, P. R. (1993): The Economics of Bureaucracy, in: Gemmell, N. (Hrsg.), The Growth of the Public Sector, Aldershot, S. 86-102

Cusack, T. R. (1988): Public Expenditure Decision-Making: A Comparative Analysis, in: in: Lybeck, J. A. und Henrekson, M. (Hrsg.) Explaining the Growth of Government, Amsterdam, S. 59-89

David, P. A. und J. L. Scadding (1974): „Private savings: „Ultrarationality“, aggregation and „Denison's Law“, in: Journal of Political Economy, Vol. 82, S. 225-249

Davies, G. et al. (1994): Options for 1995: The Green Budget, IFS Commentary Nr. 46, London

De Haan, J. und Zelhorst, D. (1988): The Empirical Evidence on the Ricardian Equivalence Hypothesis, in: Kredit und Kapital, Vol. 21, Nr. 3, S. 407-420

Deutsche Bundesbank (1994): Aufkommen und ökonomische Auswirkungen des steuerlichen Zinsabschlags, in: Monatsbericht der Deutschen Bundesbank, Vol. 46, Nr. 1, S. 45-58

De Tocqueville, A. (1835): Democracy in America, (wieder abgedruckt 1965), in: Henry Reeve, Oxford World Classics, Oxford

Diamond, P. A. (1965): National Debt in a Neoclassical Growth Model, in: American Economic Review, Vol. 55, S. 1126-1150

Dilnot, A. (1995): The Assessment: The Future of the Welfare State, in: Oxford Review of Economic Policy, Vol. 11, Nr. 3, S. 1-10

Dilnot, A. und Walker, I. (Hrsg.) (1989): The Economics of Social Security, Oxford,

Disney, R. und Webb, S. (1989): Market Failure in occupational sick pay, in: The Economics of Social Security, Dilnot, A. und Walker, I. (Hrsg.), Oxford, S. 52-65

Djaroueh, M. (1994): The British Privatization Program, Diss., Bamberg

Domar, E. D. (1946): Capital Expansion, Rate of Growth, and Employment, in: Econometrica, Vol. 14, April, S. 137-147

Dornbusch, R. (1988): The European Monetary System, the Dollar and the Yen, in: Giavazzi, F., Micossi, S. und Miller, M. (Hrsg.) The European Monetary System, Cambridge, S. 23-41

Dow, J. C. R. und Saville, I. D. (1988): A critique of monetary policy. Theory and British Experience, Oxford

Dowds, L. und Ahrendt, D. (1995): Fear of Crime, in: Jowell, R., Curtice, J., Park, A., Brook, L. und Ahrendt, D. (Hrsg.), British Social Attitudes, the 12th Report, S. 19-41

Downs, A. (1957): An Economic Theory of Democracy, New York

Drazen, A. (1978): Government Debt, Human Capital, and Bequests in a Life Cycle Model, in: Journal of Political Economy, Vol. 86, S. 505-516

Drazen, A. (1981): Inflation and capital accumulation under a finite horizon, in: Journal of Monetary Economics, Vol. 8, S. 247-260 
Drazen, A. (1989): Monetary policy, capital controls and seignorage in an open economy, in: Cecco, de M. und Giovannini, A. (Hrsg.) A European Central Bank? Perspectives on monetary unification after ten years of the EMS, Cambridge, S. 13-32

Dunsire, A. und Hood, C. (1989): Cutback management in public bureaucracies. Popular theories and observed outcomes in Whitehall, Cambridge

„The Economist“ Newspaper, verschiedene Ausgaben, London

Eichengreen, B. (1990): One money for Europe? Lessons from the US currency union, in: Economic Policy, Nr. 10, S. 118-187

Eichengreen, B. und Lindert, P. H. (Hrsg.) (1989): The International Debt Crisis in Historical Perspective, Cambridge, MA

Eichengreen, B. und von Hagen, J. (1996): Fiscal Policy and Monetary Union: Is there a tradeoff between federalism and budgetary restrictions?, NBER Working Paper Nr. 5517

Eltis, W. (1993) Classical Economics, Public Expenditure and Growth, Oxford

Emerson, M., Gros, D., Italiener, A., Pisani-Ferry, J. und Reichenbach, H. (1991): Ein Markt eine Währung: potentielle Nutzen und Kosten der Errichtung einer Wirtschafts- und Währungsunion; eine Studie der Kommission der Europäischen Gemeinschaft, Generaldirektion Wirtschaft und Finanzen, Bonn

Europäische Gemeinschaften (1990): Die Steuern im Europäischen Binnenmarkt, Amt für amtliche Veröffentlichungen der Europäischen Gemeinschaften, Luxemburg

Eurostat Jahrbuch 1995: Europa im Blick der Statistik 1983-1993

Feinstein, C. H. (1988): Economic growth since 1870: Britain's performance in international perspective, in: Oxford Review of Economic Policy, Vol. 4, Nr 1, S. 1-13

Feldstein, M. (1982): Consumer deficits and aggregate demand, in: Journal of Monetary Economics, Vol. 9, S. 1-20

Feldstein, M. und Horioka, C. (1980): Domestic saving and international capital flows, in: Economic Journal, Vol. 90, S. 314-329

Fellner, W. (1983) Comment on Peacock, A. (1983): in: Giersch, H. (Hrsg.): Reassessing the Role of Government in the Mixed Economy, Tübingen, S. 25-30

Fels, G. und v. Furstenberg, G. M. (Hrsg.) (1989): A Supply-side Agenda for Germany, Berlin

Ferguson, J. M. (Hrsg.), (1964): Public Debt and Future Generations, Chapel Hill

Financial Times, London, verschiedene Ausgaben

Fischer, S. und Modigliani, F. (1978): Towards an Understanding of the Costs of Inflation, in: Weltwirtschaftliches Archiv, Bd. CXIV, S. 810-833

Fischer, S. (1982): Seignorage and the Case for a National Money, in: Journal of Political Economy, Vol.90, S. 295-313

Fitz Gerald, J. D. (1990): The distortionary effects of taxes on trade in border areas: the Republic of Ireland-UK border, in: Winkler, G. (Hrsg.), Tax harmonisation and Financial Liberalisation in Europe, New York

Fletcher, M. (1992): Poll tax, a short sharp history, Reading

Francke, H.-H. und Kotz, H.-G. (1994): Einführung, in: Francke, H.-H., Ketzel, E. und Kotz, H.H. (Hrsg.), Handbuch des öffentlichen Kredits, Stuttgart, S. 9-30

Frey, B. S. und Kirchgässner, G. (1994): Demokratische Wirtschaftspolitik, München 
Frey, B. S. und Schneider, F. (1981): Recent Research on Empirical Politic-Economic Models, in: Hibbs Jr. D. A. und Fassbender, H. (Hrsg.), Contemporary Political Economy, Amsterdam

Friedman, M. (1971): Government Revenue from Inflation, in: Journal of Political Economy, Vol. 79 II, S. 846-856

Friedman, M. (1987): The Quantity Theory of Money, in: The New Palgrave. A dictionary of Economics, Vol. 4, Eatwell, J., Milgate, M. und Newman, P. (Hrsg.), Oxford, S. 3-20

Fry, G. K. (1988): The Thatcher Government, the Financial Management Initiative and the „New Civil Service“, in: Public Administration, Vol. 66(1), S. 1-20

Fullerton, D. (1982): On the Possibility of an Inverse Relationship between Tax Rates and Government Revenues, in: Journal of Public Economics, Vol. 19, S. 3-22

Gandenberger, O. (1970): Öffentlicher Kredit und Einkommensverteilung, in: Finanzarchiv, Vol. 29 , S. $1-16$

Gandenberger, O. (1981): Theorie der offentlichen Verschuldung, in: Handbuch der Finanzwissenschaft, III, 3. Aufl., Tübingen, S. 3-49

Gardner, E. H. (1992): Taxes on Capital Income: A Survey, in: Kopits, G. (Hrsg) (1992): Tax Harmonization in the European Community. Policy Issues and Analysis, International Monetary Fund Occasional Paper Nr. 94

Garfinkel, M. R. (1989): What Is an „Acceptable“ Rate of Inflation? - A Review of the Issues, in: The Federal Reserve Bank of St. Louis, Review, July/August, S. 3-15

Gaysford, S. (1994): The duties helping France but damaging Britain, Parliamentary Brief, Vol. 2, S. 24

Gebauer, W. (1982): Realzins, Inflation und Kapitalzins. Eine Neuinterpretation des FisherTheorems, Berlin

Gemmell, N. (1990): The Public Sector: Definition and Measurement Issues, in: Gemmell, N. (Hrsg.):The Growth of the Public Sector, Aldershot, S. 1-14

Giovannini, A. (1989): National tax systems versus the European capital market, in: Economic Policy, Nr. 9, S. 345- 386

Giavazzi, F. (1990): The EMS: Lessons from Europe and Perspectives in Europe, in: Ferri, P. (Hrsg.) Prospects for the European Monetary System, S. 36-55

Giles, C. und Ridge, M. (1993): The Impact on Households of the 1993 Budget and the Council Tax, in: Fiscal Studies, Vol. 14, Nr. 3, S. 1-20

Giles, C. und Johnson, P. (1994): Tax Reform in the UK and Changes in the Progressivity of the Tax System, 1985-1995, in: Fiscal Studies, Vol. 15, Nr. 3, S. 64-86

Glynn, J. (1987): Public Sector Financial Control and Accounting, Oxford

Gokhale, J., Raffelhüschen, B. und Walliser, J. (1995): The Burden of German Unification: A Generational Accounting Approach, in: Finanzarchiv, N.F. Bd. 52, S. 141-165

Goodman, A. und Webb, S. (1994): For Richer, For Poorer: The Changing Distribution of Income in the UK, 1961-91, IFS Commentary Nr. 42, London

Gosling, A., Machin, S. und Meghir (1994): What has happened to Men's Wages since the Mid60s?, in: Fiscal Studies, Vol. 15, Nr. 4, S. 63-87

Gowland, D. H. (1990): Debt management in the UK, in: Gowland, D. H. (Hrsg.): International Bond Markets, London 
Grilli, V. (1989): Seignorage in Europe, in: Cecco, de M. und Giovannini, A. (Hrsg.) A European Central Bank? Perspectives on monetary unification after ten years of the EMS, Cambridge, S. 53-79

Grilli, V., Masciandara, D. und Tabellini, G. (1991): Political and monetary institutions and public financial policies in the industrial countries, in: Economic Policy, Nr. 13, S. 342-392

Gros, D. und Thygesen, N. (1992): European Monetary Integration: From the European Monetary System to European Monetary Union, London

Haan, de J., Sterks, C. G. M. und Kam, de C. A. (1992): Towards budget discipline: an economic assessment of the possibilities for reducing national deficits in the run-up to EMU, Economic Papers Nr. 99, Brussels

Hagemann, R. P., Jones, B. und Montador, B. (1988): Tax Reform in OECD Countries: Motives, Constaints and Practice, in: OECD Economic Studies, Nr. 10, S. 185-226

Haliassos, M. und Tobin, J. (1990): The Macroeconomics of Government Finance, in: Friedman, B. M. und Hahn, F. H. (Hrsg.), Handbook of Monetary Economics, Vol.II, S. 889-959.

Hamlin, A. (1993): Public Expenditure and Political Process, in: Gemmell, N. (Hrsg.), The Growth of the Public Sector, Aldershot, S. 72-85

Haskel, J. und Jackman, R. (1988): Long-term unemployment in Britain and the effects of the Community programme, in: Oxford Bulletin of Economics and Statistics, Vol. 50, Nr. 4, S. $379-408$

Heller, P. (1981): Diverging Trends in the Shares of Nominal and Real Government Expenditure in GDP: Implications for Policy, in: National Tax Journal, Vol. 34, Nr. 1, A. 61-74

Helm, D. und Smith, S. (1987): The Assesment: Decentralisation and the Economics of Local Government, in: Oxford Review of Economic Policy, Vol. 3, Nr. 2, S. i-xxi

Hennings, K. H. (1985): Ist „Thatcherism“ angebotsorientierte Wirtschaftspolitik?, in: Staat und Beschäftigung: Angebots- und Nachfragepolitik in Theorie und Praxis, Buttler, F., Kühl, J. und Rahmann, B. (Hrsg.), Institut für Arbeitsmarkt- und Berufsforschung der Bundesanstalt für Arbeit, Nürnberg, S. 139-184

Hibberd, J. (1993a): Trends in public expenditure: 1978-79 to 1992-93, in: CSO Treasury Bulletin, Vol. 4 (2) S. 20-32.

Hibberd, J. (1993b): Trends in public sector capital spending, in: CSO Treasury Bulletin, Vol. 4 (2) S. 55-74.

Hills, J. (1989): Counting the Family Silver: The Public Sector's Balance Sheet 1957 to 1987, in: Fiscal Studies, Vol. 10, Nr. 2, S. 66-85

Hills, J. (1995a): Joseph Rowntree Foundation Inquiry into Income and Wealth, Vol. 1., York

Hills, J. (1995b): Joseph Rowntree Foundation Inquiry into Income and Wealth, Vol. 2: A summary of the evidence, York

Hills, J. (1996): Tax Policy: Are there still choices?, in: Options for Britain: A strategic policy review, Halpern, D., Wood, S. White, S. und Cameron, G. (Hrsg.), Aldershot, Dartmouth, S. 71-98

HMSO (1944), Employment Policy, Command Paper Nr. 6527, London

HMSO (1979) The Government's Expenditure Plan 1980-81, Command Paper Nr. 7746, London

HMSO (1980a): Memoranda on Monetary Policy, House of Commons and Civil Service Committee, sess. 1979/80, Vol. 1, London

HMSO (1980b): Memoranda on Monetary Policy, House of Commons Treasury and Civil Service Committee, sess. 1979-80, Vol. 2, London 
HMSO (1980c): Monetary control, Command Paper Nr. 7858, London

Hoover, K. D. (1988): The new classical macroeconomics, a sceptical enquiry, Oxford

Hotelling, H. (1929): Stability in competition, in: Economic Journal, Vol. 39, S. 41-57

Hubbard, R. G. und Judd, K. L. (1986): Liquidity Constraints, Fiscal Policy, and Consumption, in: Brookings Papers on Economic Activity, Vol. 13. S. 1-50

Huber, B. (1990): Staatsverschuldung und Allokationseffizienz: Eine theoretische Analyse, Baden-Baden

Hume, D. (1741) (wieder abgedruckt 1907): Essays. Moral, Political, and Literary, Vol. 1, London

Humes IV, S. (1991): Local Governance and National Power, London

Hutchinson, G. und Church, A. (1989): Wages, Unions, the Youth Training Scheme und the Young Workers Scheme, in: Scottish Journal of Political Economy, Vol.36, Nr. 2

Hutton, W. (1995): The State We're In, London

IMF (International Monetary Fund) (1995a): Saving in a Growing World Economy, in: World Economic Outlook, October, Washington, S. 67-89

IMF (International Monetary Fund) (1995b): Financial Market Turmoil and Economic Policies in Industrial Countries, in: World Economic Outlook, October, Washington, S. 32-43

IMF (International Monetary Fund) (1996): Fiscal Challenges Facing Industrial Countries, in: World Economic Outlook, May, Washington, S. 44-62

Ingham, G. (1984): Capitalism Divided? The City and industry in British social development, New York

Issing, O. (1993): Einführung in die Geldtheorie, 9. Aufl., München

Jackson, P. M. (1980): The Public Expenditure Cuts: Rationale and Consequences, in: Fiscal Studies, Vol. 1, S. 66-77

Jackson, P. M. (1991): Public Expenditure, in: Labour's Economic Policies, Artis, M. und Cobham, D. (Hrsg.), Manchester, S. 73-87

Jackson, P. M. (1996): A Qualified Defence, in: Seldon, A. (Hrsg.) (1996): Re-Privatising Welfare: After the Lost Century, Institute of Economic Affairs, IEA Readings Nr. 45, London, S. $1-9$

Jenkins, S. P. (1995): Accounting for Inequality Trends: Decomposition Analyses for the UK, 1971-1986, in: Economica, Vol. 62, Nr. 245, S. 29-63

Jenkins, S. P. und Cowell, F. A. (1993): Dwarfs und giants in the 1980s: The UK income distribution and how it changed, Department of Economics Discussion Paper 93-03, Swansea

Jenkins, S. P. und Cowell, F. A. (1994): Dwarfs und giants in the 1980s: trends in the UK income distribution, in: Fiscal Studies, Vol. 15, Nr. 1, S. 99-118

Johnson, P. und Webb, S. (1992): The treatment of housing in official low income statistics, in: Journal of the Royal Statistical Society, Vol. A. S. 273-290

Johnson, P. und Webb, S. (1993): Explaining the growth in UK income inequality, in: Economic Journal, Vol. 103, S. 429-443

Kaldor, Lord (1966): Causes of the Slow Rate of Growth in the United Kingdom, Cambridge

Kath, D. (1972): Die verschiedenen Ansätze der Zinsstrukturtheorie, in: Kredit und Kapital, Vol. 6, S. 28-71.

Kay, J. A. und King, M. A. (1990): The British Tax System, 5. Aufl., Oxford 
Kay, J. A. und Thompson, D. J. (1986): Privatisation: A Policy in Search of a Rationale, in: Economic Journal, Vol. 96, Nr. 1, S. 18-32

Keegan, W. (1984): Mrs Thatcher's Economic Experiment, London

Keen, M. (1987): Welfare Effects of Commodity Tax Harmonisation, in: Journal of Public Economics, Vol. 33, S. 107-14

Kennedy, N. O. (1989): Inflation-adjusted sectoral saving and financial balances, in: Bank of England Quarterly Bulletin, S. 246-251

Keynes, J. M. (1922): Lecture to the Institute of Bankers, 29.11.1922, in: The Collected Writings of John Maynard Keynes, Vol. XIX, London

Keynes, J. M. (1923): A Tract on Monetary Reform, in: The Collected Writings of John Maynard Keynes, Vol. IX, London

Keynes, J. M. (1936): The General Theory of Employment, Interest and Money, in: The Collected Writings of John Maynard Keynes, Vol. VII, London

King, M. (1994): Monetary Policy in the UK, in: Fiscal Studies, Vol. 15, Nr. 3, S. 109-128

Kitterer, W. (1986): Sind Steuern und Staatsverschuldung äquivalente Instrumente zur Finanzierung der Staatsausgaben?, in: Kredit und Kapital, Vol. 19, S. 271-291

Kitterer, W. (1988): Staatsverschuldung und intertemporale Allokation, in: Jahrbuch für Nationalökonomie und Statistik, Bd. (Vol.) 204/4, S. 346-363

Klein, M. und M. J. M. Neumann (1990): Seignorage: What Is It and Who Gets It?: in: Weltwirtschaftliches Archiv, Vol. 126, S. 205-221

Kopits, G. (Hrsg) (1992): Tax Harmonization in the European Community. Policy Issues and Analysis, International Monetary Fund Occasional Paper Nr. 94

Kotlikoff, L. J. und Summers, L. H. (1981): The Role of Intergenerational Transfers in Aggregate Capital Accumulation, in: Journal of Political Economy, Vol. 89, S. 706-732

Laidler, D. E. W. (1975): Essays on Money and Inflation, Manchester

Leibenstein, H: (1966): Allokative Efficiency vs. X-Efficiency, in: American Economic Review, Vol. 56, Juni, S. 392-415

Leiderman, L. und Blejer, M. I. (1988): Modeling and Testing Ricardian Equivalence: A Survey, in: IMF Staff Papers, Vol. 35, Nr. 1, S. 1-35

Lerner, A. P. (1948): The Burden of the National Debt, wieder abgedruckt in: Ferguson, J. M. (Hrsg.), (1964): Public Debt and Future Generations, Chapel Hill, S. 16-19

Lindbeck, A. (1985): Redistribution Policy and the Expansion of the Public Sector, in: Journal of Public Economics, Vol. 28, S. 309-328

Lipsey, D. (1994): Do we really want more public spending, in: Jowell, R., Curtice, J., Brook, L. und Ahrendt, D. (Hrsg.), British Social Attitudes: the 11 th Report, Aldershot, Dartmouth

Lockwood, B., de Meza, D. und Myles, G. (1994): On the European Union VAT Proposals: The Superiority of Origin over Destination Taxation, in: Fiscal Studies, Vol. 16, Nr. 1, S. 1-77

Lybeck, J. A. (1986): The Growth of Government in Developed Economies, Aldershot

Lybeck, J. A. (1988): Comparing Government Growth Rates: The Non-Institutional vs. the Institutional Approach, in: Lybeck, J. A. und Henrekson, M. (Hrsg.) Explaining the Growth of Government, Amsterdam, S. 29-47

Mansoor, A. M. (1993): Budgetary Impact of Privatisation, in: Blejer, M. I. und Cheasty, A. (Hrsg.) How to measure the fiscal deficit, Washington, S. 345-362

Maynard, G. (1988): The Economy under Mrs Thatcher, Oxford 
McLure, C. (1986): Tax competition: Is what's good for the private goose also good for the public gander, in: National Tax Journal, Nr. 39, S. 341-348

Meen, G. (1988): International comparisons of the UK's long-run economic performance, in: Oxford Review of Economic Policy, Vol. 4, Nr. 1, S. xxii-xli

Meltzer, A. H. und Richard, S. F. (1981): A Rational Theory of the Size of Government, Journal of Political Economy, Vol. 89, S. 914-927

Meyer, L. H. (Hrsg.) (1981): The Supply-Side Effects of Economic Policy, Hingham, MA

Michaelis, J. (1989): Optimale Finanzpolitik im Modell überlappender Generationen, Frankfurt

Michaelis, J. (1993): On the Superneutrality of Money, in: Jahrbuch für Nationalökonomie und Statistik, Vol. 212, Bd. 3-4, S. 257-269

Michl, T. R. (1990): Debt, deficits, and the distribution of income, in: Journal of Post Keynesian Economics, Vol.13, Nr. 3, S. 351-365

Miller, M. (1982): Inflation-adjusting the Public Sector Financial Deficit, in: J. Kay (Hrsg.) The 1982 Budget, Oxford, S. 48-74

Minford, P. (1980): A Rational Expectations Model of the United Kingdom under Fixed and Floating Exchange Rates, in: Journal of Monetary Economics,

Mitchell, B. R. und Deane, P. (1962): Abstract of British Historical Statistics, Cambridge

Modigliani, F. (1961): Long-run implications of alternative fiscal policies and the burden of the national debt, wieder abgedruckt in: Ferguson, J. M. (Hrsg.), (1964): Public Debt and Future Generations, Chapel Hill, S. 107-149

Mueller, D. C. (1989): Public Choice II, Cambridge

Mueller, D. C. und Murrell, P. (1985): Interest Groups and the Political Economy of Government Size, in: Peacock, A. und Forte, F. (Hrsg.), Public Expenditure and Government Growth, Oxford, S. 13-36

Mullard, M. (1987): The Politics of Public Expenditure, London

Musgrave, R. A. (1985): Excess bias in the nature of budget growth, in: Journal of Public Economics, Vol. 28 S. 227-308

Musgrave, R. A., Musgrave, P. B. und Kullmer, L. (1994): Die öffentlichen Finanzen in Theorie und Praxis, Bd. 1., 6. Aufl., Tübingen

NEDC (National Economic Development Council) (1985): Investment in the public sector built infrastructure, London

Neumark Committee (1963): Report of the fiscal and financial committee, The EEC reports on tax harmonization (International Bureau of Fiscal Documentation), Amsterdam

Niskanen, W. A. (1971): Bureaucracy and Representative Government, New York

Niskanen, W. A. (1973): Bureaucracy: Servant or Master?, Hobart Paperback, Nr. 5, Institute of Economic Affairs, London

Niskanen, W. A. (1978): Deficits, government spending, and inflation. What is the evidence?, in: Journal of Monetary Economics, Vol. 4, Nr. 3, S. 591-602

Nordhaus, W. D. (1989): Alternative approaches to the political business cycle, in: Brookings Papers of Economic Activity, Nr. 2, S. 1-49

North, D. C. (1985): The Growth of Government in the United States: An Economic Historian's Perspective, in: Journal of Public Economics, Vol. 28 S. 383-399 
Oberhauser, A. (1970): Gewinnorientierte Einkommenspolitik und Staatsverschuldung, in: Theorie und Praxis des finanzpolitischen Interventionismus, Haller, H., Kullmer, L. Shoup, C. S. und Timm, H. (Hrsg.), Tübingen, S. 529-550

Oates, W. E. (1972): Fiscal Federalism, New York

Olson, M., Jr. (1965): The Logic of Collective Action, Cambridge

Olson, M., Jr. (1982): The Rise and Decline of Nations: Economic Growth, Stagflation, and Social Rigidities, New Haven

OECD (Organisation for Economic Co-operation and Development) (1989): Public Sector Efficiency, in: Economic Surveys, United Kingdom, S. 63-96

OECD (Organisation for Economic Co-operation and Development) (1993a): Wirtschaftsausblick, Ist das Realzinsniveau „hoch“, Juni, Paris, S. 27-34

OECD (Organisation for Economic Co-operation and Development) (1993b): Wirtschaftsausblick, Einige Maßnahmen zur qualitativen Verbesserung der Staatsausgaben, Dezember, Paris, S. 37-49

OECD (Organisation for Economic Co-operation and Development) (1993c): Public Sector Pay Trends: Report on Pilot Study, Paris

OECD (Organisation for Economic Co-operation and Development) (1993d): Wirtschaftsausblick, Juni

OECD (Organisation for Economic Co-operation and Development) (1995): Wirtschaftsausblick, Juni, Paris

OECD (Organisation for Economic Co-operation and Development) (1996): Wirtschaftsausblick, Juni, Paris

Orphanides, A. und Solow, R. J. (1990): Money, Inflation and Growth, in: Friedman, B. M. und Hahn, F. H. (Hrsg.), Handbook of Monetary Economics, Vol.I, Amsterdam, S. 224-261

Oxley, H., Maher, M., Martin, J. P., Nicoletti, G. und Alonso-Gamo, P. (1990): The Public Sector: Issues for the 1990s, OECD Working Paper Nr. 90.

Ott, D. J. und Ott, A. F. (1965): Budget Balance and Equilibrium Income, in: The Journal of Finance, Vol. XX, March, S. 71-77

Ott, A. F. (1993): Public Sector Budget: A Comparative Study, Aldershot

Paldam, M. (1981): A Preliminary Survey of the Theories and Findings on Vote and Popularity Functions, in: European Journal of Political Research, Nr. 9, S. 181-199

Paldam, M. und Zeuthen, H. E. (1988): The Expansion of the Public Sector in Denmark - A Post Festum?, in: Lybeck, J. A. und Henrekson, M. (Hrsg.) Explaining the Growth of Government, Amsterdam, S. 299-326

Peacock, A. T. (1979): The Economic Analysis of Government and Related Themes, Oxford

Peacock, A. (1983): Reducing Government Expenditure Growth: A British View, in: Giersch, H. (Hrsg.): Reassessing the Role of Government in the Mixed Economy, Tübingen, S. 1-24

Peacock, A. (1985): Macro-economic Controls of Spending as a Device for Improving Efficiency in Government, in: Peacock, A. und Forte, F. (Hrsg.), Public Expenditure and Government Growth, Oxford, S. 143-156

Peacock, A. (1989): The Rise and Fall of the Laffer Curve, in: Bös, D und Felderer, B. (Hrsg), The Political Economy of Progressive Taxation, Berlin

Peltzman, S. (1980): The Growth of Government, in: Journal of Law and Economics, Nr. 23, S. 209-287 
Perotti, R. (1992): Increasing Returns to Scale, Politics, and the Timing of Stabilization, Columbia University, Department of Economics Discussion Paper Nr. 596

Persson, T. (1985): Deficits and intergenerational welfare in open economies, in: Journal of International Eocnomics, Vol. 19, S. 67-84

Persson, T. und Svensson, L. E. O. (1989): Why a stubborn conservative would run a deficit: policy with time-inconsistent preferences, in: Quarterly Journal of Economics, Vol. 104, S. $325-345$

Persson, T. und Tabellini, G. (1993): Does Centralization increase the size of Government, Paper prepared for the 8th Annual Congress of the European Economic Association in Helsinki, 27-29.08.93

Peters, K. J. (1990): Inflation-adjusted sectoral saving and financial balances, in: BoEQB, Vol. 30 , Nr. 2, S. 228-231

Phelps, E. S. (1961): The Golden Rule of Accumulation: A Fable for Growthmen, in: American Economic Review, Vol. 51, S. 638-643

Phelps, E. S. (1965): Second Essay on the Golden Rule of Accumulation, in: American Economic Review, Vol. 55, S. 793-814

Pigou, A. C. (1928): A study in Public Finance, London

Plumb, J.H. (1956): Sir Robert Walpole, Vol. I. The Making of a Statesman, London

Pommerehme, W. W. und Kirchgässner, G. (1988): Gesamtwirtschaftliche Effizienz, gesellschaftliche Umverteilung und Wachstum der Staatstätigkeit: Ein Überblick, in: Zimmerman, H. (Hrsg.), Die Zukünft der Staatsfinanzierung, Stuttgart, S. 206-241

Poterba, J. M. und Summers, L. H. (1987): Finite Lifetimes and the effects of budget deficits on national saving, in: Journal of Monetary Economics, Vol. 20, S. 369-391

Pryke, R. (1982): The comparative performance of public and private enterprise, in: Fiscal Studies, Vol. 3, S. 68-81

Pyle, D. L. (1989): Tax Evasion and the Black Economy, London

Pyle, D. L. (1993): The Economics of Taxpayer Compliance, in: Jackson, P. M. (Hrsg.) Current Issues in Public Sector Economics, London, S. 58-93

Redmond, G. und Sutherland, H. (1995): How has tax and social security policy changed since 1978? A distributional analysis, Microsimulation Unit Discussion Paper, Department of Applied Economics, University of Cambridge

Renaud, P. A. S. und van Winden, F. A. A. M. (1988): Fiscal behaviour and the Growth of Government in the Netherlands, in: Lybeck, J. A. und Henrekson, M. (Hrsg.) Explaining the Growth of Government, Amsterdam, S. 133-156

Richter, W. F. und Wiegard, W. (1993): Zwanzig Jahre „Neue Finanzwissenschaft“, Teil II, in: Zeitschrift für Wirtschafts- und Sozialwissenschaften, Vol. 113, S. 337-400

Ricardo, D. (1820): Funding System, in: Sraffa, P. (Hrsg.) (1962): The Works and Correspondence of David Ricardo, Volume IV Pamphlets and Papers 1815-1823, Cambridge

Ricardo, D ([1821]1951): The Works and Correspondence of David Ricardo, Sraffa, P. (Hrsg.), Cambridge

Roubini, N. und Sachs, J. (1989a): Political and Economic Determinants of Budget Deficits in the Industrial Economies, in: European Economic Review, Vol. 33, S. 903-938

Roubini, N. und Sachs, J. (1989b): Government spending and budget deficits in the industrial countries, in: Economic Policy, Nr. 8, S. 100-132 
Rowley, C. K. (1979): Buying out the Obstructors?, in: The Taming of Government, Institute of Economic Affairs, IEA Readings Nr. 21, London, S. 107-123

Rürup, B. (1982): Begrenzungskriterien der staatlichen Kreditaufnahme, in: Bombach, G. u. a. (Hrsg): Möglichkeiten und Grenzen der Staatstätigkeit, Schriftenreihe des wirtschaftwissenschaftlichen Seminars Ottobeuren, Bd 11, Tübingen, S. 603-666

Ryan, P. (1996): Factor shares and Inequality in the UK, in: Oxford Review of Economic Policy, Vol. 12, Nr. 1, S. 106-126

Sachs, J. D. (1989): Introduction, in: Sachs, J. D. (Hrsg.) Developing Country Debt and the World Economy, Chicago, S. 1-33

Safford, J. (1994): Vereinigtes Königreich, in: Francke, H.-H., Ketzel, E. und Kotz, H.-H. (Hrsg.), Handbuch des öffentlichen Kredits, Stuttgart, S. 421-449

Sargent, T. J. und Wallace, N (1981): Some Unpleasant Monetarist Arithmetic, wieder abgedruckt (1986): in: Sargent, T. J. (Hrsg.): Rational Expectations and Inflation, New York, S. $173-210$

Saunders, P. (1993): Recent Trends in the Size and Growth of Government in OECD Countries, in: Gemmell, N. (Hrsg.):The Growth of the Public Sector, Aldershot, S. 17-33

Schelling, T. C. (1982): Establishing Credibility: Strategic Considerations, in: American Economic Review, Vol. 72, Nr. 2, S. 77-89

Schlesinger, H., Weber, M. und Ziebarth, G. (1993): Staatsverschuldung - ohne Ende? Zur Rationalität und Problematik des offentlichen Kredits, Darmstadt

Seater, J. J. (1993): Ricardian Equivalence, in: Journal of Economic Literature, Vol. 31, S. 142191

Seldon, A. (Hrsg.) (1996): Re-Privatising Welfare: After the Lost Century, Institute of Economic Affairs, IEA Readings Nr. 45, London

Shaw, G. K. (1983): Fiscal Policy under the First Thatcher Administration 1979-1983, in: Finanzarchiv NF 41, S. 312-342

Shaw, G. K. (1984): Fiscal Policy: The Third Thatcher Administration 1987-1990 and the Thatcher Legacy, in: Finanzarchiv NF 51, S. 75-94

Sidrauski, M. (1967): Inflation and economic growth, in: Journal of Political Economy, Vol. 75, S. 796-810

Siegel, J. J. (1979): Inflation-induced distortions in government and private savings statistics, in: Review of Economics and Statistics, Vol. 61, S. 83-90

Sinn, G. und Sinn, H.-W. (1991): Kaltstart. Volkswirtschaftliche Aspekte der deutschen Wiedervereinigung, Tübingen

Sinn, S. (1992): Saving-Investment Correlations and Capital Mobility: on the Evidence from Annual Data, in: Economic Journal, Vol. 102, S. 1171-1183

Skidelsky, R. (1995): Public Finance and Education, in: Oxford Review of Economic Policy, Vol. 11, Nr. 3, S. 59-66

Slemrod, J. (1990): Optimal Taxation and Optimal Tax Systems, in: Journal of Economic Perspectives, Vol. 4 Nr. 1, S. 157-178

Smith, A. (1776): An Inquiry into the nature and causes of the wealth of nations, Edinburgh, Neuauflage 1976, Hrsg. Campbell, R. H. und Skinner, A. S., Oxford

Smith, P. (1991): Lessons from the British Poll Tax Disaster, in: National Tax Journal, Vol. XLIV, Nr. 4, S. 421-436 
Smith, S. (1991): Distributional Issues in Local Taxation, in: The Economic Journal, Vol. 10, Mai, S. 585-599

Smith, S. (1994): Fiscal Anarchy in the UK: Lessons from the Poll Tax, in: IFS Update, Spring/Summer, S. 3-4

Smith, S. und Ridge, M. (1990): Local Government Finance: The 1990 Reforms, Institute for Fiscal Studies, IFS Commentary Nr. 22, London

Spahn, P. B. und Kaiser, H. (1991): Tax Harmonization or Tax Competition as Means to Integrate Western Europe, in: Konjunkturpolitik, Nr. 37, S. 1-43

Spaventa, L. (1989): Seignorage: Old and New Policy Issues, in: European Economic Review, 33, S. 557-563

Stiglitz, J. (1986): The General Theory of Tax Avoidance, NBER Working Paper Nr. 1868

Stiglitz, J. und Weiss, A. (1981): Credit Rationing in Markets with Imperfect Information, in: American Economic Review, Vol. 71, S. 393-411

Stoker, G. (1988): The Politics of Local Government, London

Summers, L. H. (1983): The Non-Adjustment of Nominal Interest Rates, in: Essays in Honor of A. Okun, Brooking Institution, Washington, D.C.

Tabellini, G. (1991): The politics of intergenerational redistribution, in: Journal of Political Economy, Vol. 99, Nr. 2, S. 335-357

Tabellini, G. und Alesina, A. (1990): Voting on the Budget Deficit, in: American Economic Review, Vol. 80, S. 37-52

Tanzi, V. (1985): Monetary Policy and Expenditure Control, in: Peacock, A. und Forte, F. (Hrsg.), Public Expenditure and Government Growth, Oxford, S. 157-168

Tarschys, D. (1975): The Growth of Public Expenditures: Nine Modes of Explanation, in: Scandinavian Political Studies, Vol. 10, S. 9-31

Taylor, C. T. und Threadgold, A. R. (1979): „Real“ national saving and its sectoral composition, Bank of England Discussion Paper Nr. 6

Taylor-Gooby, P. (1995): Comfortable, marginal and excluded: Who should pay higher taxes for a better welfare state? , in: Jowell, R., Curtice, J., Park, A., Brook, L. und Ahrendt, D. (Hrsg.), British Social Attitudes, the 12th Report, S. 1-17

Temperton, P. (1986): A Guide to UK Monetary Policy, London

Tiebout, C. (1956): A Pure Theory of Local Expenditure, in: Journal of Political Economy, Vol.64, S. 416-424

Tobin, J. (1969): A General Equilibrium Approach to Monetary Theory, in: Journal of Money, Credit and Banking, Vol.1, S. 15-29

Tobin, J. (1981): Vermögensakkumulation und wirtschaftliche Aktivität (Originaltitel): Asset Accumulation and Economic Activity), München

Tobin, J. und Buiter, W. H. (1990): Long-run effects of fiscal and monetary policy on aggregate demand, in: Buiter, W. H. (1990): Principles of Budgetary and Financial Policy, S.252-286

Tullock, G. (1959): Some Problems of Majority Voting, in: Journal of Political Economy, Vol. 67 , S. $571-9$

Tullock, G. (1974): Dynamic Hypothesis on bureaucracy, in: Public Choice, Vol. 19, S. 127-131

Van Velthoven, B., Verbon, H. A. A. und F. A. A. M. van Winden (1993): The political economy of public debt: a survey, in: Verbon, H. A. A. und F. A. A. M. van Winden (Hrsg.): The Political Economy of Government Debt, Amsterdam, S. 3-36 
Viren, M. (1986): The relationship between interest rates and inflation during the prewar period. Some empirical evidence, in: Economic letters, Vol. 20, Nr. 1, S. 23-27

Von Hagen, J. (1992): Budgeting Procedures and Fiscal Performance in the European Communities, Economic Papers of the Commission of the European Communities, Nr. 96

Wagner, A. (1883): Finanzwissenschaft, 3. Aufl., Teil 1, Leipzig und Heidelberg

Wagner, A. (1892): Grundlegung der politischen Ökonomie, 3. Aufl., Teil 1, Leipzig

Walters, A. A. (1983): On Britain's Conservative Reform 1979, in: Focus, The Fraser Institute, Vancouver

Walters, A. A. (1985): Deficits in the United Kingdom, in: Essays in Contemporary Economic Problems: The Economy in Deficit, Cagan, P. (Hrsg.), AEI, Washington, S. 147-162

Walters, A. A. (1986): Britain's Economic Renaissance: Margaret Thatcher's Reforms 1979-1984, Oxford

Waud, R. N. (1986): Tax aversion and the Laffer curve, in: Scottish Journal of Political Economy, Vol. 33, Nr 3, S. 213-227

Waud, R. N. (1988): Tax aversion, optimal tax rates, and indexation, in: Public Finance, Nr 2, S. 310 325

Webb, S. (1996): Social Security Policy in a Changing Labour Market, in: Oxford Review of Economic Policy, Vol. 11, Nr. 3. S. 11-26

Weichenrieder, A. (1996): Fighting international tax avoidance: the case of Germany, in: Fiscal Studies, Vol. 17, Nr. 1, S. 37-58

Westaway, T. (1992): The fiscal dimension of 1992, in: Swann, D. (Hrsg.), The single European market and beyond, London, S. 81-105

Wilcox, S. (1994): Making the most of council housing, in: Fiscal Studies, Vol. 15, Nr. 1, S. 4463

Wilhelm, M. O. (1996): Bequest behavior and the Effect of Heirs' Earnings: Testing the Altruistic Model of Bequest, in: American Economic Review, Vol. 86, Nr. 4, S. 874-892

World Bank (1988): World Development Report, Washington D.C.

Yarrow, G. (1986): Privatization in theory and practice, in: Economic Policy Nr. 2, S. 324-377

Yotsuzaka, T. (1987): Ricardian Equivalence in the presence of capital market imperfections, in: Journal of Monetary Economics, Vol. 20, S. 411-436

Zeldes, S. P. (1989): Consumption and Liquidity Constraints: An Empirical Investigation, in: Journal of Political Economy, Vol. 97, S. 305-346 


\section{FINANZWISSENSCHAFTLICHE SCHRIFTEN}

Band 1 Werner Steden: Finanzpolitik und Einkommensverteilung. Ein Wachstums- und Konjunkturmodell der Bundesrepublik Deutschland. 1979.

Band 2 Rainer Hagemann: Kommunale Finanzplanung im föderativen Staat. 1976.

Band 3 Klaus Scherer: Maßstäbe zur Beurteilung von konjunkturellen Wirkungen des öffentlichen Haushalts. 1977.

Band 4 Brita Steinbach: "Formula Flexibility" - Kritische Analyse und Vergleich mit diskretionärer Konjunkturpolitik. 1977.

Band 5 Hans-Georg Petersen: Personelle Einkommensbesteuerung und Inflation. Eine theoretisch-empirische Analyse der Lohn- und veranlagten Einkommensteuer in der Bundesrepublik Deutschland. 1977.

Band 6 Friedemann Tetsch: Raumwirkungen des Finanzsystems der Bundesrepublik Deutschland. Eine Untersuchung der Auswirkungen der Finanzreform von 1969 auf die Einnahmenposition der untergeordneten Gebietskörperschaften und ihrer regionalpolitischen Zieladäquanz. 1978.

Band 7 Wilhelm Pfähler: Normative Theorie der fiskalischen Besteuerung. Ein methodologischer und theoretischer Beitrag zur Integration der normativen Besteuerungstheorie in der Wohlfahrtstheorie. 1978.

Band 8 Wolfgang Wiegard: Optimale Schattenpreise und Produktionsprogramme für öffentliche Unternehmen. Second-Best Modelle im finanzwirtschaftlichen Staatsbereich. 1978.

Band 9 Hans P. Fischer: Die Finanzierung des Umweltschutzes im Rahmen einer rationalen Umweltpolitik. 1978.

Band 10 Rainer Paulenz: Der Einsatz finanzpolitischer Instrumente in der Forschungs- und Entwicklungspolitik. 1978.

Band 11 Hans-Joachim Hauser: Verteilungswirkungen der Staatsverschuldung. Eine kreislauftheoretische Inzidenzbetrachtung. 1979.

Band 12 Gunnar Schwarting: Kommunale Investitionen. Theoretische und empirische Untersuchungen der Bestimmungsgründe kommunaler Investitionstätigkeit in NordrheinWestfalen 1965-1972. 1979.

Band 13 Hans-Joachim Conrad: Stadt-Umland-Wanderung und Finanzwirtschaft der Kernstädte. Amerikanische Erfahrungen, grundsätzliche Zusammenhänge und eine Fallstudie für das Ballungsgebiet Frankfurt am Main. 1980.

Band 14 Cay Folkers: Vermögensverteilung und staatliche Aktivität. Zur Theorie distributiver Prozesse im Interventionsstaat. 1981.

Band 15 Helmut Fischer: US-amerikanische Exportförderung durch die DISC-Gesetzgebung. 1981.

Band 16 Günter Ott: Einkommensumverteilungen in der gesetzlichen Krankenversicherung. Eine quantitative Analyse. 1981.

Band 17 Johann Hermann von Oehsen: Optimale Besteuerung. (Optimal Taxation). 1982.

Band 18 Richard Kössler: Sozialversicherungsprinzip und Staatszuschüsse in der gesetzlichen Rentenversicherung. 1982.

Band 19 Hinrich Steffen: Zum Handlungs- und Entscheidungsspielraum der kommunalen Investitionspolitik in der Bundesrepublik Deutschland. 1983.

Band 20 Manfred Scheuer: Wirkungen einer Auslandsverschuldung des Staates bei flexiblen Wechselkursen. 1983. 
Band 21 Christian Schiller: Staatsausgaben und crowding-out-Effekte. Zur Effizienz einer Finanzpolitik keynesianischer Provenienz. 1983.

Band 22 Hannelore Weck: Schattenwirtschaft: Eine Möglichkeit zur Einschränkung der öffentlichen Verwaltung? Eine ökonomische Analyse. 1983.

Band 23 Wolfgang Schmitt: Steuern als Mittel der Einkommenspolitik. Eine Ergänzung der Stabilitätspolitik? 1984.

Band 24 Wolfgang Laux: Erhöhung staatswirtschaftlicher Effizienz durch budgetäre Selbstbeschränkung? Zur Idee einer verfassungsmäßig verankerten Ausgabengrenze. 1984.

Band 25 Brita Steinbach-van der Veen: Steuerinzidenz. Methodologische Grundlagen und empirisch-statistische Probleme von Länderstudien. 1985.

Band 26 Albert Peters: Ökonomische Kriterien für eine Aufgabenverteilung in der Marktwirtschaft. Eine deskriptive und normative Betrachtung für den Allokationsbereich. 1985.

Band 27 Achim Zeidler: Möglichkeiten zur Fortsetzung der Gemeindefinanzreform. Eine theoretische und empirische Analyse. 1985.

Band 28 Peter Bartsch: Zur Theorie der längerfristigen Wirkungen 'expansiver' Fiskalpolitik. Eine dynamische Analyse unter besonderer Berücksichtigung der staatlichen Budgetbeschränkung und ausgewählter Möglichkeiten der öffentlichen Defizitfinanzierung. 1986.

Band 29 Konrad Beiwinkel: Wehrgerechtigkeit als finanzpolitisches Verteilungsproblem. Möglichkeiten einer Kompensation von Wehrungerechtigkeit durch monetäre Transfers. 1986.

Band 30 Wolfgang Kitterer: Effizienz- und Verteilungswirkungen des Steuersystems. 1986.

Band 31 Heinz Dieter Hessler: Theorie und Politik der Personalsteuern. Eine Kritik ihrer Einkommens- und Vermögensbegriffe mit Blick auf die Leistungsfähigkeitstheorie. 1994.

Band 32 Wolfgang Scherf: Die beschäftigungspolitische und fiskalische Problematik der Arbeitgeberbeiträge zur Rentenversicherung. Eine Auseinandersetzung mit der Kritik an der lohnbezogenen Beitragsbemessung. 1987.

Band 33 Andreas Mästle: Die Steuerunion. Probleme der Harmonisierung spezifischer Gütersteuern. 1987.

Band 34 Günter Ott: Internationale Verteilungswirkungen im Finanzausgleich der Europäischen Gemeinschaften. 1987.

Band 35 Heinz Haller: Zur Frage der zweckmäßigen Gestalt gemeindlicher Steuern. Ein Diskussionsbeitrag zur Gemeindesteuerreform. 1987.

Band 36 Thomas Kuhn: Schlüsselzuweisungen und fiskalische Ungleichheit. Eine theoretische Analyse der Verteilung von Schlüsselzuweisungen an Kommunen. 1988.

Band 37 Walter Hahn: Steuerpolitische Willensbildungsprozesse in der Europäischen Gemeinschaft. Das Beispiel der Umsatzssteuer-Harmonisierung. 1988.

Band 38 Ulrike Hardt: Kommunale Finanzkraft. Die Problematik einer objektiven Bestimmung kommunaler Einnahmemöglichkeiten in der gemeindlichen Haushaltsplanung und im kommunalen Finanzausgleich. 1988.

Band 39 Jochen Michaelis: Optimale Finanzpolitik im Modell überlappender Generationen. 1989.

Band 40 Bernd Raffelhüschen: Anreizwirkungen der sozialen Alterssicherung. Eine dynamische Simulationsanalyse. 1989.

Band 41 Berend Diekmann: Die Anleihe- und Darlehenstransaktionen der Europäischen Gemeinschaften. 1990.

Band 42 Helmut Kaiser: Konsumnachfrage, Arbeitsangebot und optimale Haushaltsbesteuerung. Theoretische Ergebnisse und mikroökonometrische Simulation für die Bundesrepublik Deutschland. 1990. 
Band 43 Rüdiger von Kleist: Das Gramm-Rudman-Hollings-Gesetz. Ein gescheiterter Versuch der Haushaltskonsolidierung. 1991.

Band 44 Rolf Hagedorn: Steuerhinterziehung und Finanzpolitik. Ein theoretischer Beitrag unter besonderer Berücksichtigung der Hinterziehung von Zinserträgen. 1991.

Band 45 Cornelia S. Behrens: Intertemporale Verteilungswirkungen in der gesetzlichen Krankenversicherung der Bundesrepublik Deutschland. 1991.

Band 46 Peter Saile: Ein ökonomischer Ansatz der Theorie der intermediären Finanzgewalten Die Kirchen als Parafisci. 1992.

Band 47 Peter Gottfried: Die verdeckten Effizienzwirkungen der Umsatzsteuer. Eine empirische allgemeine Gleichgewichtsanalyse. 1992.

Band 48 Andreas Burger: Umweltorientierte Beschäftigungsprogramme. Eine Effizienzanalyse am Beispiel des "Sondervermögens Arbeit und Umwelt". 1992.

Band 49 Jeanette Malchow: Die Zuordnung verteilungspolitischer Kompetenzen in der Europäischen Gemeinschaft. Eine Untersuchung aufgrund einer Fortentwicklung der ökonomischen Theorie des Föderalismus. 1992.

Band 50 Barbara Seidel: Die Einbindung der Bundesrepublik Deutschland in die Europäischen Gemeinschaften als Problem des Finanzausgleichs. 1992.

Band 51 Ralph Wiechers: Markt und Macht im Rundfunk. Zur Stellung der öffentlich-rechtlichen Rundfunkanstalten im dualen Rundfunksystem der Bundesrepublik Deutschland. 1992.

Band 52 Klaus Eckhardt: Probleme einer Umweltpolitik mit Abgaben. 1993.

Band 53 Oliver Schwarzkop: Die Problematik unterschiedlicher Körperschaftsteuersysteme innerhalb der EG. 1993.

Band 54 Thorsten Giersch: Bergson-Wohlfahrtsfunktion und normative Ökonomie. 1993.

Band 55 Li-Fang Chou: Selbstbeteiligung bei Arzneimitteln aus ordnungspolitischer Sicht. Das Beispiel der Bundesrepublik Deutschland. 1993.

Band 56 Harald Schlee: Einkommensteuerliche Behandlung von Transferzahlungen. Zur Neuordnung der Familienbesteuerung sowie der Besteuerung von Versicherungsleistungen und Sozialtransfers. 1994.

Band 57 Alexander Spermann: Kommunales Krisenmanagement. Reaktionen baden-württembergischer Stadtkreise auf steigende Sozialhilfekosten und Einnahmenausfälle (198092). 1993.

Band 58 Otto Roloff / Sibylle Brander / Ingo Barens / Claudia Wesselbaum: Direktinvestitionen und internationale Steuerkonkurrenz. 1994.

Band 59 Claudia Wesselbaum-Neugebauer: Internationale Steuerbelastungsvergleiche. 1994.

Band 60 Stephanie Miera: Kommunales Finanzsystem und Bevölkerungsentwicklung. Eine Analyse des kommunalen Finanzsystems vor dem Hintergrund der sich abzeichnenden Bevölkerungsentwicklung am Beispiel Niedersachsens unter besonderer Berücksichtigung des Landkreises Wolfenbüttel und seiner Gemeinden. 1994.

Band 61 Wolfgang Scherf: Die Bedeutung des kaldorianischen Verteilungsmechanismus für die gesamtwirtschaftlichen Wirkungen der staatlichen Neuverschuldung. 1994.

Band 62 Rainer Volk: Vergleich der Vergünstigungseffekte der verschiedenen investitionsfördernden Maßnahmen. 1994.

Band 63 Hans-Georg Napp: Kommunale Finanzautonomie und ihre Bedeutung für eine effiziente lokale Finanzwirtschaft. 1994. 2., unveränderte Auflage 1994.

Band 64 Bernd Rahmann / Uwe Steinborn / Günter Vornholz: Empirische Analyse der Autonomie lokaler Finanzwirtschaften in der Europäischen Gemeinschaft. 1994. 
Band 65 Carsten Kühl: Strategien zur Finanzierung der Altlastensanierung. 1994.

Band 66 Stephan Boll: Intergenerationale Umverteilungswirkungen der Fiskalpolitik in der Bundesrepublik Deutschland. Ein Ansatz mit Hilfe des Generational Accounting. 1994.

Band 67 Karl Justus Bernhard Neumärker: Finanzverfassung und Staatsgewalt in der Demokratie. Ein Beitrag zur konstitutionellen Finanztheorie. 1995.

Band 68 Christian Haslbeck: Zentrale versus dezentrale Internalisierung externer Effekte bei unvollständiger Information. 1995.

Band 69 Regina Müller: Horizontale oder vertikale Transfers zur Durchsetzung eines horizontalen Finanzausgleichs. 1995.

Band 70 Christian Hockenjos: Öffentliche Sporttörderung in der Bundesrepublik Deutschland. Darstellung und finanztheoretische Analyse. 1995.

Band 71 Manfred Rosenstock: Die Kontrolle und Harmonisierung nationaler Beihilfen durch die Kommission der Europäischen Gemeinschaften. 1995.

Band 72 Christian Rüsch: Wohnungsbau- und Wohneigentumspolitik im Rahmen der Einkommensteuer. Eine Analyse unter steuersystematischen, verteilungspolitischen und fiskalischen Aspekten. 1996.

Band 73 Stephan Winters: Die kollektive Vorsorge für den Pflegefall im Alter. Eine Untersuchung am Beispiel der gesetzlichen Pflegeversicherung in den Niederlanden. 1996.

Band 74 Knut Blind: Allokationsineffizienzen auf Sicherheitsmärkten: Ursachen und Lösungsmöglichkeiten. Fallstudie: Informationssicherheit in Kommunikationssystemen. 1996.

Band 75 Barbara Petrick-Rump: Ökonomische Wirkungen von Steueramnestien. Untersuchung konkreter Erfahrungen ausgewählter Länder mit dem Einsatz von Steueramnestien anhand eines effizienten Steueramnestieprogramms. 1996.

Band 76 Georg Hirte: Effizienzwirkungen von Finanzausgleichsregelungen. Eine Empirische Allgemeine Gleichgewichtsanalyse für die Bundesrepublik Deutschland. 1996.

Band 77 Ulrike Kirchhoff: Die meinland-pfälzischen Gemeinden im System des Finanzausgleichs. 1996.

Band 78 Kerstin Keil: Der soziale Mietwohnungsbau: Mängel und Alternativen. 1996.

Band 79 Bernhard Manzke: Kinderlastenausgleich versus verstärkte Einwanderung. Alternative Ansätze zur langfristigen Sicherung der Gesetzlichen Rentenversicherung. 1997.

Band 80 Hariolf M. Wenzler: Institutionenökonomik und öffentliche Finanzkontrolle. Eine Analyse am Beispiel der Europäischen Union. 1997.

Band 81 Joachim Nagel: Supply-Side Policy in den USA. Eine theoretische und empirische Analyse der angebotsorientierten Wirtschaftspolitik Reagans unter besonderer Berücksichtigung steuerlicher Aspekte. 1997.

Band 82 Heinz Lampert: Krise und Reform des Sozialstaates. 1997.

Band 83 Monika Hanswillemenke / Bernd Rahmann: Zwischen Reformen und Verantwortung für Vollbeschäftigung. Die Finanz- und Haushaltspolitik der sozial-liberalen Koalition von 1969 bis 1982. 1997.

Band 84 Berthold Fürst: Die Maastrichter Budgetkriterien im Konflikt mit der Verschuldungsautonomie der deutschen Gebietskörperschaften. 1997.

Band 85 Burkhard Pahnke: Einkommensorientierte Förderung des sozialen Mietwohnungsbaues. Bestandsaufnahme und Kritik. 1998.

Band 86 Judith Safford: Staatsverschuldung im Vereinigten Königreich. Die öffentliche Verschuldung unter der Konservativen Regierung von 1979-1994. Ursachen und Auswirkungen. 1998. 
Judith Safford - 978-3-631-75199-2

Downloaded from PubFactory at 01/11/2019 07:12:45AM

via free access 
Heinz Lampert

\section{Krise und Reform des Sozialstaates}

Frankfurt/M., Berlin, Bern, New York, Paris, Wien, 1997. 104 S., 1 Tab.

Finanzwissenschaftliche Schriften. Bd. 82

Verantwortlicher Herausgeber: Alois Oberhauser

ISBN 3-631-32186-4 • br. DM 39.-*

Die Entstehung und die Entwicklung des Sozialstaates ist eine der bedeutendsten Errungenschaften der Neuzeit. Aufgrund gravierender Veränderungen der wirtschaftlichen und politischen Rahmenbedingungen und der Alterung der Bevölkerungen ist sein wirtschaftliches Fundament gefährdet, aufgrund ideologischer Neuentwicklungen auch seine sozialphilosophische Grundlage. Diese auch den Sozialstaat Bundesrepublik bedrohende Problematik greift der Verfasser auf. Nach einer Darstellung der Entstehung, Entwicklung und Erfolge des Sozialstaates werden seine Grenzen und die aktuelle Diskussion der Krise des Sozialstaates in Deutschland dargestellt. Nach einer Erörterung der Prinzipien des Umbaues des Sozialstaates und einer Skizze der bereits verwirklichten Reformschritte werden der Reformbedarf, die politischen Voraussetzungen für eine Sozialstaatsreform und Reformempfehlungen herausgearbeitet.

Aus dem Inhalt: Entstehung, Entwicklung und Erfolge des Sozialstaats . Grenzen des Sozialstaats - Die Sozialstaatskrise im Licht der aktuellen Diskussion - Prinzipien des Sozialstaatsumbaus - Verwirklichte Reformschritte - Der Reformbedarf - Politische Voraussetzungen der Reform . Reformschritte und Empfehlungen

Frankfurt/M - Berlin - Bern - New York - Paris - Wien

Auslieferung: Verlag Peter Lang AG

Jupiterstr. 15, CH-3000 Bern 15

Telefax (004131) 9402131

*inklusive Mehrwertsteuer

Preisänderungen vorbehalten 
Judith Safford - 978-3-631-75199-2

Downloaded from PubFactory at 01/11/2019 07:12:45AM

via free access 